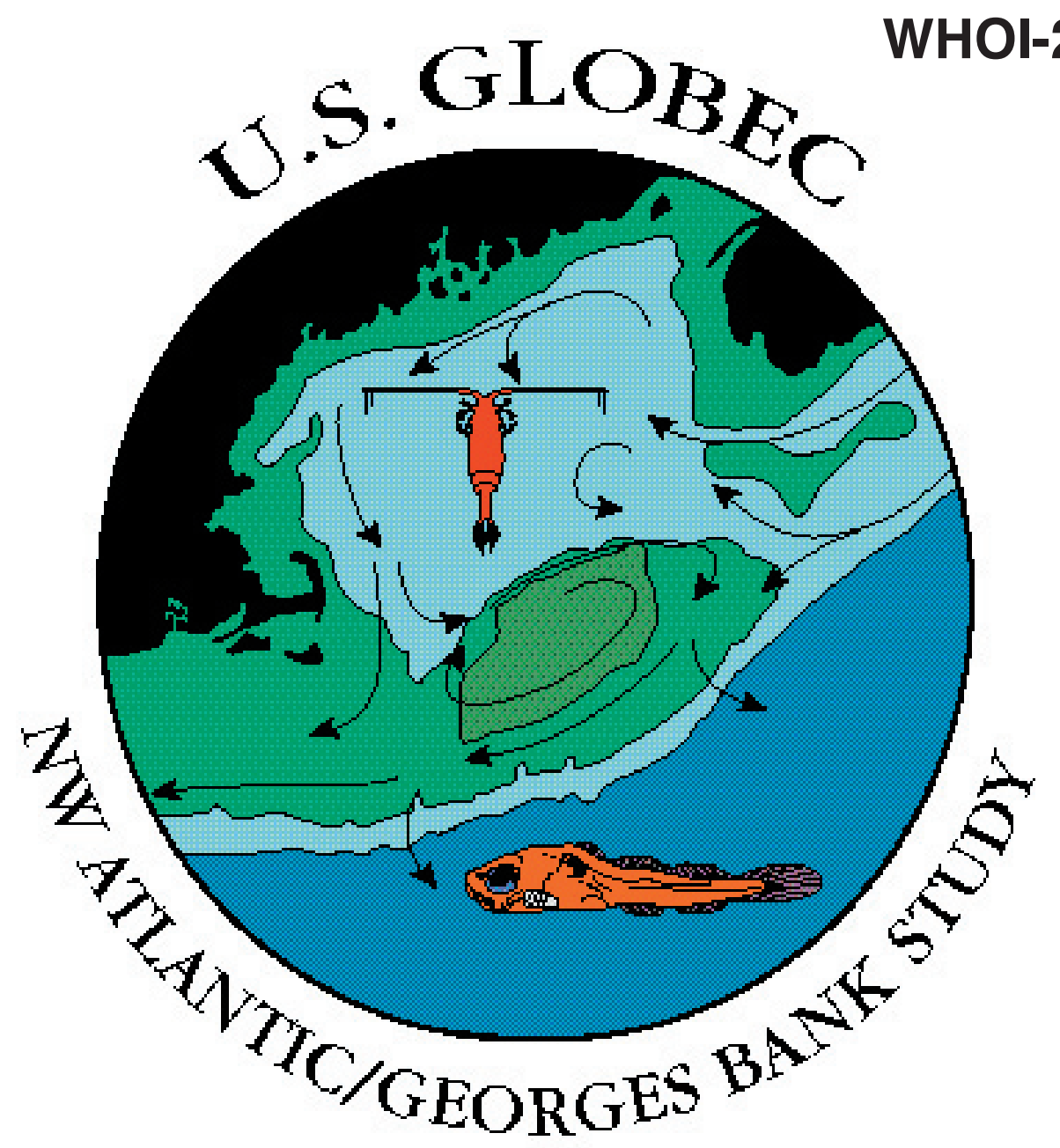

\title{
The 1995 Georges Bank Stratification Study and Moored Array Measurements
}

by

C. Alessi, R. Beardsley, M. Caruso, J. Churchill, J. Irish, S. Lentz, R. Limeburner, R. Werner, R. Weller, A. Williams, W. Williams

Woods Hole Oceanographic Institution, Woods Hole, MA 02543

J. Manning

NOAA/NMFS, Woods Hole, MA 02543

P. Smith

Bedford Institute of Oceanography, Dartmouth, N.S., Canada B2Y 4A2

August 2001

\section{Technical Report}

Funding was provided by the National Science Foundation under Grant Numbers OCE-98-06379 and OCE-98-06445.

Approved for public release; distribution unlimited. 


\section{The 1995 Georges Bank Stratification Study and Moored Array Measurements}

by

C. Alessi, R. Beardsley, M. Caruso, J. Churchill, J. Irish, S. Lentz, R. Limeburner, R. Werner, R. Weller, A. Williams, W. Williams

Woods Hole Oceanographic Institution

Woods Hole, MA 02543

J. Manning

NOAA/NMFS

Woods Hole, MA 02543

P. Smith

Bedford Institute of Oceanography

Dartmouth, N.S., Canada B2Y 4 A2

August 2001

\section{Technical Report}

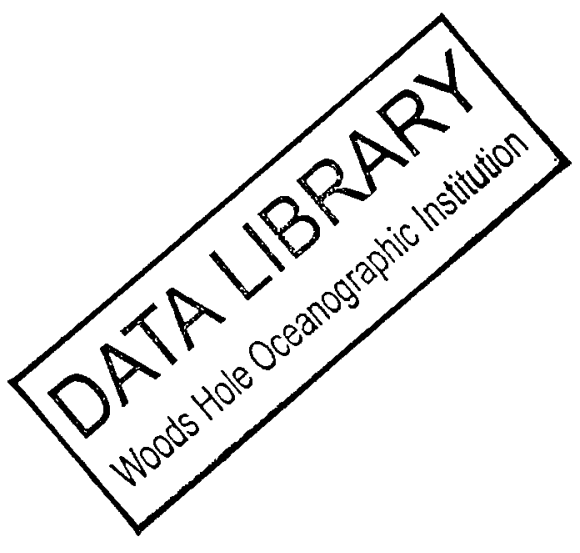

Funding was provided by the National Science Foundation under Grant Numbers OCE-98-06379 and OCE-98-06445.

Reproduction in whole or in part is permitted for any purpose of the United States Government. This report should be cited as Woods Hole Oceanog. Inst. Tech. Rept., WHOI-2001-11.

Approved for public release; distribution unlimited.

Approved for Distribution:

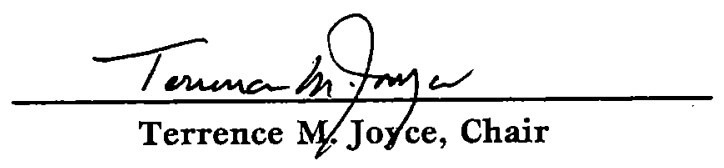

Department of Physical Oceanography 


\section{Table of Contents}

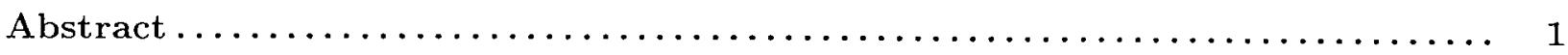

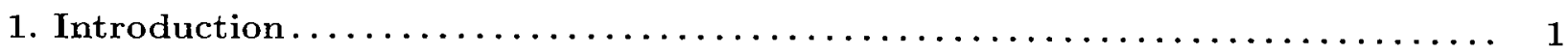

1.1 U.S. GLOBEC Northwest Atlantic/Georges Bank Program ........... 1

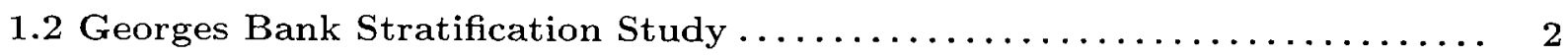

1.3 Objectives and Organization of This Report................. 4

2. Stratification Study Moored Array $\ldots \ldots \ldots \ldots \ldots \ldots \ldots \ldots \ldots \ldots \ldots \ldots \ldots \ldots$

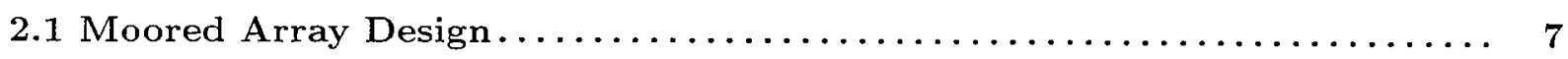

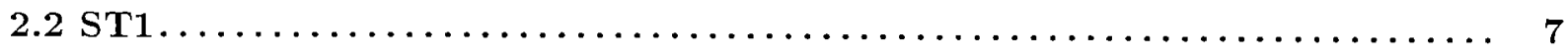

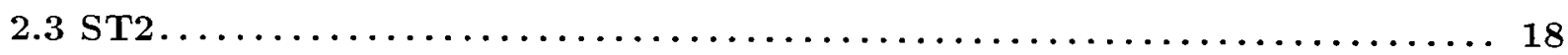

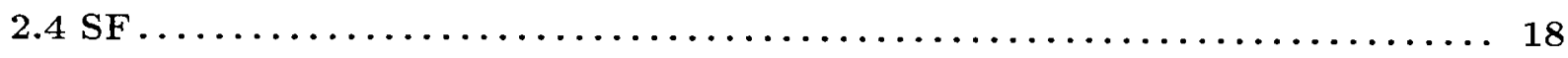

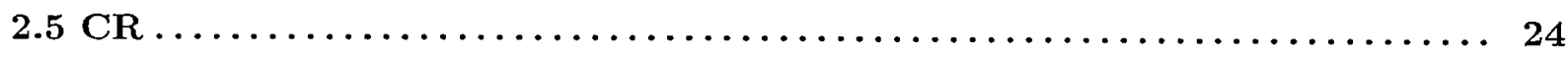

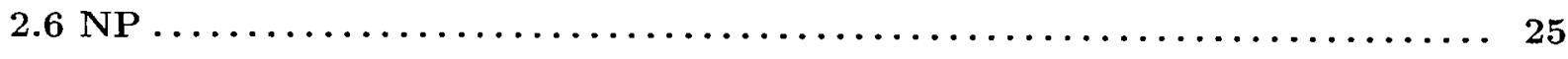

2.7 NDBC Environmental Buoys........................... 25

3. Summary of GBSS Moored Instrumentation $\ldots \ldots \ldots \ldots \ldots \ldots \ldots \ldots \ldots \ldots$

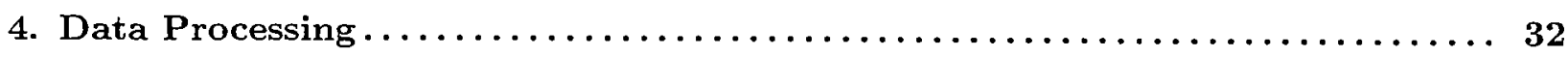

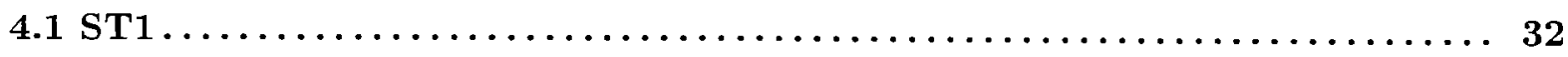

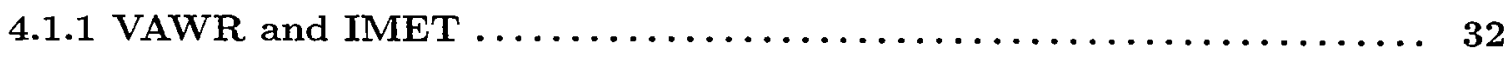

4.1.1.1 Wind Speed and Direction $\ldots \ldots \ldots \ldots \ldots \ldots \ldots \ldots \ldots \ldots \ldots \ldots$

4.1.1.2. Incident Short-Wave Radiation $\ldots \ldots \ldots \ldots \ldots \ldots \ldots \ldots \ldots$ 
4.1.1.3. Incident Long-Wave Radiation ................. 33

4.1.1.4 Barometric Pressure....................... 35

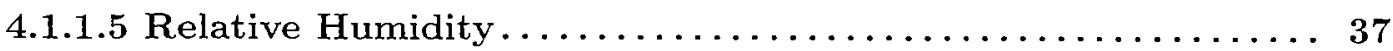

4.1.1.6 Air Temperature $\ldots \ldots \ldots \ldots \ldots \ldots \ldots \ldots \ldots \ldots \ldots \ldots \ldots \ldots \ldots \ldots$

4.1.1.7 Water Temperature $\ldots \ldots \ldots \ldots \ldots \ldots \ldots \ldots \ldots \ldots \ldots \ldots \ldots .39$

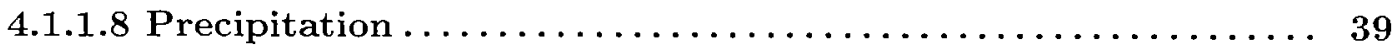

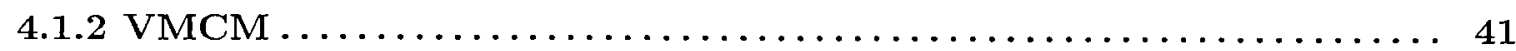

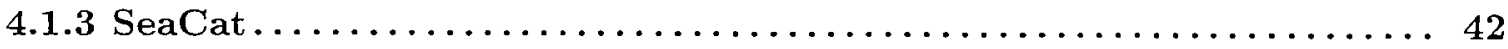

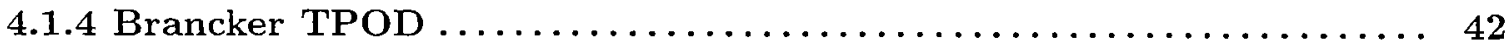

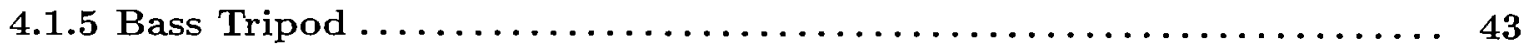

4.1.5.1 Velocity $\ldots \ldots \ldots \ldots \ldots \ldots \ldots \ldots \ldots \ldots \ldots \ldots \ldots \ldots \ldots \ldots \ldots \ldots$

4.1.5.2 Temperature $\ldots \ldots \ldots \ldots \ldots \ldots \ldots \ldots \ldots \ldots \ldots \ldots \ldots \ldots \ldots, 43$

4.1.5.3 Optical Backscatter ...................... 44

4.1.5.4 Camera $\ldots \ldots \ldots \ldots \ldots \ldots \ldots \ldots \ldots \ldots \ldots \ldots \ldots \ldots \ldots \ldots \ldots \ldots$

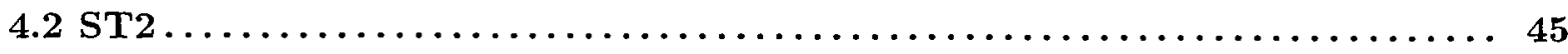

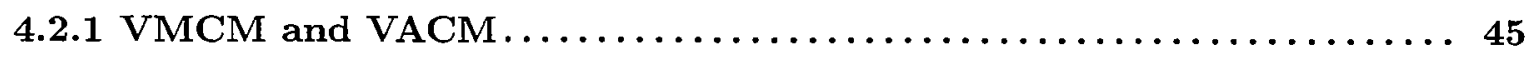

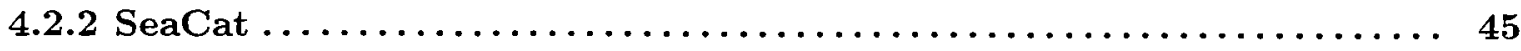

4.2.3 Brancker TPOD $\ldots \ldots \ldots \ldots \ldots \ldots \ldots \ldots \ldots \ldots \ldots \ldots \ldots \ldots \ldots \ldots \ldots \ldots \ldots$

4.2.4 VEMCO Minilog TPOD $\ldots \ldots \ldots \ldots \ldots \ldots \ldots \ldots \ldots \ldots \ldots \ldots \ldots \ldots \ldots$

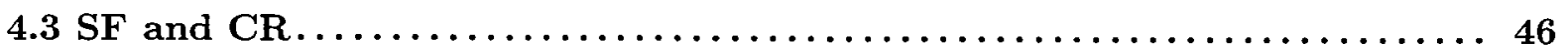




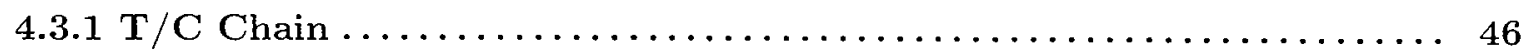

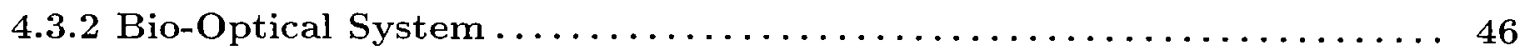

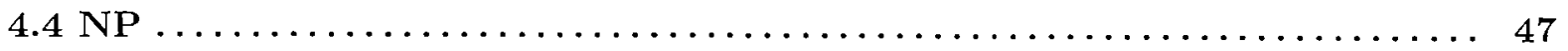

$4.4 .1 \mathrm{RCM} \ldots \ldots \ldots \ldots \ldots \ldots \ldots \ldots \ldots \ldots \ldots \ldots \ldots \ldots \ldots \ldots \ldots \ldots \ldots \ldots \ldots$

4.4 .2 TG $\ldots \ldots \ldots \ldots \ldots \ldots \ldots \ldots \ldots \ldots \ldots \ldots \ldots \ldots \ldots \ldots \ldots \ldots \ldots \ldots \ldots \ldots$

4.5 NDBC Environmental Buoys $\ldots \ldots \ldots \ldots \ldots \ldots \ldots \ldots \ldots \ldots \ldots \ldots \ldots \ldots$

5. Data Availability on the World Wide Web: http://globec.whoi.edu ....... 48

6. 1995 GBSS Mooring Cruise Reports....................... 48

7. Basic Statistics of GBSS Moored Array Time Series ................ 49

8. Plots of GBSS Moored Array Time Series .................... 49

Acknowledgments $\ldots \ldots \ldots \ldots \ldots \ldots \ldots \ldots \ldots \ldots \ldots \ldots \ldots \ldots \ldots \ldots \ldots \ldots \ldots \ldots \ldots \ldots .49$

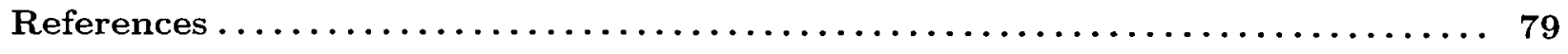

\section{List of Tables}

Table 1. Summary of Instrumented Mooring Positions............... 5

Table 2. WHOI VAWR Specifications $\ldots \ldots \ldots \ldots \ldots \ldots \ldots \ldots \ldots \ldots \ldots \ldots \ldots \ldots$

Table 3. WHOI IMET Specifications $\ldots \ldots \ldots \ldots \ldots \ldots \ldots \ldots \ldots \ldots \ldots \ldots \ldots \ldots .9$

Table 4 . VAWR and IMET Sensor Heights $\ldots \ldots \ldots \ldots \ldots \ldots \ldots \ldots \ldots \ldots \ldots \ldots$

Table 5. WHOI VMCM Specifications $\ldots \ldots \ldots \ldots \ldots \ldots \ldots \ldots \ldots \ldots \ldots \ldots \ldots$

Table 6. Sea-Bird SeaCat Specifications $\ldots \ldots \ldots \ldots \ldots \ldots \ldots \ldots \ldots \ldots \ldots \ldots \ldots \ldots$

Table 7. Brancker TPOD Specifications $\ldots \ldots \ldots \ldots \ldots \ldots \ldots \ldots \ldots \ldots \ldots \ldots \ldots$ 
Table 8. WHOI BASS Measurement Periods and Sensor Heights .......... 13

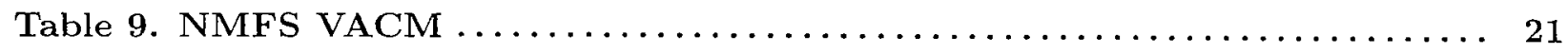

Table 10. SF and CR Sensor Specifications $\ldots \ldots \ldots \ldots \ldots \ldots \ldots \ldots \ldots \ldots \ldots \ldots \ldots$

Table 11. WHOI Bio-Optical Package Specifications $\ldots \ldots \ldots \ldots \ldots \ldots \ldots \ldots \ldots \ldots 22$

Table 12. Bottom-Mounted ADCP $/ \mathrm{P} / \mathrm{T} / \mathrm{C}$ Specifications............. 23

Table 13. PMEL Temperature Recorder Specifications $\ldots \ldots \ldots \ldots \ldots \ldots \ldots \ldots \ldots 24$

Table 14a. Aanderaa RCM Specifications $\ldots \ldots \ldots \ldots \ldots \ldots \ldots \ldots \ldots \ldots \ldots \ldots \ldots$

Table 14b. Aanderaa Tide Gauge Specifications $\ldots \ldots \ldots \ldots \ldots \ldots \ldots \ldots \ldots \ldots \ldots 24$

Table 15. NDBC Environmental Buoy Specifications $\ldots \ldots \ldots \ldots \ldots \ldots \ldots \ldots \ldots \ldots$

Table 16. NDBC Buoy Sensor Heights $\ldots \ldots \ldots \ldots \ldots \ldots \ldots \ldots \ldots \ldots \ldots \ldots \ldots \ldots \ldots$

Table 17. Summary of Moored Instrumentation Deployed $\ldots \ldots \ldots \ldots \ldots \ldots \ldots$

Table 18. GBSS Mooring Cruise Reports..................... 48

Table 19. Monthly Statistics of GBSS Moored Array Time Series........... 51

Table 20. Monthly Hourly-Averaged Statistics.................. 57

Table 21. Monthly Salinity Hourly-Averaged Statistics $\ldots \ldots \ldots \ldots \ldots \ldots \ldots \ldots \ldots 71$

\section{List of Figures}

Figure 1. GLOBEC Fish Paradigm $\ldots \ldots \ldots \ldots \ldots \ldots \ldots \ldots \ldots \ldots \ldots \ldots \ldots \ldots \ldots$

Figure 2. The GBSS Moored Array $\ldots \ldots \ldots \ldots \ldots \ldots \ldots \ldots \ldots \ldots \ldots \ldots \ldots \ldots \ldots$

Figure 3. Timeline for GBSS Moored Array $\ldots \ldots \ldots \ldots \ldots \ldots \ldots \ldots \ldots \ldots \ldots \ldots$

Figure 4. GBSS Mooring Separation $\ldots \ldots \ldots \ldots \ldots \ldots \ldots \ldots \ldots \ldots \ldots \ldots \ldots \ldots$ 
Figure 5. Map of ST1 Mooring Positions $\ldots \ldots \ldots \ldots \ldots \ldots \ldots \ldots \ldots \ldots \ldots \ldots$

Figure 6A. Schematic of $3-\mathrm{m}$ Discus Buoy $\ldots \ldots \ldots \ldots \ldots \ldots \ldots \ldots \ldots \ldots \ldots \ldots$

Figure $6 \mathrm{~b}$. Top View of Discus Tower $\ldots \ldots \ldots \ldots \ldots \ldots \ldots \ldots \ldots \ldots \ldots \ldots \ldots \ldots$

Figure 7 A. ST1 Surface Mooring Diagram $\ldots \ldots \ldots \ldots \ldots \ldots \ldots \ldots \ldots \ldots \ldots \ldots$

Figure 7 B. Mid-section of Surface Mooring $\ldots \ldots \ldots \ldots \ldots \ldots \ldots \ldots \ldots \ldots \ldots \ldots$

Figure 7 C. Lower Section of Surface Mooring $\ldots \ldots \ldots \ldots \ldots \ldots \ldots \ldots \ldots \ldots \ldots$

Figure 8. ST1 Subsurface Mooring Diagram $\ldots \ldots \ldots \ldots \ldots \ldots \ldots \ldots \ldots \ldots \ldots$

Figure 9. Schematic of BASS1 $\ldots \ldots \ldots \ldots \ldots \ldots \ldots \ldots \ldots \ldots \ldots \ldots \ldots \ldots \ldots \ldots$

Figure 10. ST2 Mooring Diagram $\ldots \ldots \ldots \ldots \ldots \ldots \ldots \ldots \ldots \ldots \ldots \ldots \ldots \ldots \ldots \ldots$

Figure 11. SF Mooring Diagram $\ldots \ldots \ldots \ldots \ldots \ldots \ldots \ldots \ldots \ldots \ldots \ldots \ldots \ldots \ldots \ldots$

Figure 12. Photograph of WHOI Bio-Optical Unit.................. 23

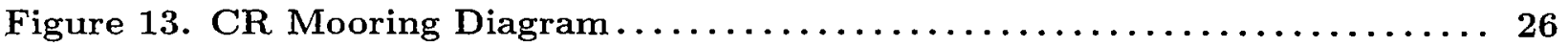

Figure 14. NP Mooring Diagram $\ldots \ldots \ldots \ldots \ldots \ldots \ldots \ldots \ldots \ldots \ldots \ldots \ldots \ldots \ldots \ldots \ldots$

Figure 15. VAWR, IMET, NDBC Wind Speed Time Series............ 34

Figure 16. Comparison of VAWR and NDBC Wind Speeds............ 34

Figure 17. VAWR and IMET Short-Wave Radiation $\ldots \ldots \ldots \ldots \ldots \ldots \ldots \ldots \ldots$

Figure 18. VAWR and IMET Hourly Short-Wave Radiation $\ldots \ldots \ldots \ldots \ldots \ldots$

Figure 19. VAWR, IMET and Clear Sky Insolation................ 36

Figure 20. VAWR and IMET Long-Wave Radiation $\ldots \ldots \ldots \ldots \ldots \ldots \ldots \ldots \ldots$

Figure 21. VAWR, IMET Long-Wave Radiation, First/Last Measurements . ... 38 
Figure 22. VAWR, IMET Long-Wave versus Short-Wave Radiation......... 38

Figure 23. VAWR and IMET Relative Humidity $\ldots \ldots \ldots \ldots \ldots \ldots \ldots \ldots \ldots \ldots$

Figure 24. VAWR and IMET Air Temperature Time Series $\ldots \ldots \ldots \ldots \ldots \ldots \ldots$

Figure 25. VAWR, IMET and SeaCat Water Temperature Time Series...... 40

Figure 26. VAWR, IMET \& SeaCat Water Temp Difference Time Series ...... 41

Figure 27. Total Precipitation Accumulation Measured $\ldots \ldots \ldots \ldots \ldots \ldots \ldots \ldots \ldots 2$

Figure 28. Plot of Monthly Means for Temperature $\ldots \ldots \ldots \ldots \ldots \ldots \ldots \ldots \ldots \ldots 77$

Figure 29. Plot of Monthly Means for Salinity $\ldots \ldots \ldots \ldots \ldots \ldots \ldots \ldots \ldots \ldots \ldots, 78$

Figure 30. Hourly Averaged Winds $\ldots \ldots \ldots \ldots \ldots \ldots \ldots \ldots \ldots \ldots \ldots \ldots \ldots \ldots \ldots$

Figure 31. ST1 Meteorology $\ldots \ldots \ldots \ldots \ldots \ldots \ldots \ldots \ldots \ldots \ldots \ldots \ldots \ldots \ldots \ldots \ldots$

Figure 32. NDBC Georges Bank Meteorology $\ldots \ldots \ldots \ldots \ldots \ldots \ldots \ldots \ldots \ldots \ldots .83$

Figure 33. $\mathrm{ST1}$ PL64 Currents $\ldots \ldots \ldots \ldots \ldots \ldots \ldots \ldots \ldots \ldots \ldots \ldots \ldots \ldots \ldots \ldots$

Figure 34. ST1 BASS $1 \& 2$ PL64 Currents $\ldots \ldots \ldots \ldots \ldots \ldots \ldots \ldots \ldots \ldots \ldots \ldots$

Figure 35. ST2 \& NP PL64 Currents....................... 86

Figure 36. ST1 Along-Bank Pl64 Currents $\ldots \ldots \ldots \ldots \ldots \ldots \ldots \ldots \ldots \ldots \ldots \ldots$

Figure 37. ST1 Cross-Bank Pl64 Currents.................... 88

Figure 38. ST1 BASS $1 \& 2$ Along/Cross-Bank PL64 Currents........... 89

Figure 39. ST2 Along/Cross-Bank PL64 Currents................. 90

Figure 40. NP Along/Cross-Bank PL64 Currents.................. 91

Figure 41. ST1 Water Temperature $\ldots \ldots \ldots \ldots \ldots \ldots \ldots \ldots \ldots \ldots \ldots \ldots \ldots \ldots .92$ 
Figure 42. ST1 BASS $1 \& 2$ Water Temperature $\ldots \ldots \ldots \ldots \ldots \ldots \ldots \ldots \ldots \ldots . . \ldots 3$

Figure 43. ST2 Water Temperature $\ldots \ldots \ldots \ldots \ldots \ldots \ldots \ldots \ldots \ldots \ldots \ldots \ldots$

Figure 44. SF Water Temperature $\ldots \ldots \ldots \ldots \ldots \ldots \ldots \ldots \ldots \ldots \ldots \ldots \ldots \ldots$

Figure 45. CR \& NP Water Temperature $\ldots \ldots \ldots \ldots \ldots \ldots \ldots \ldots \ldots \ldots \ldots$

Figure 46. Salinity: $\mathrm{ST} 1, \mathrm{ST} 2, \& \mathrm{CR} \ldots \ldots \ldots \ldots \ldots \ldots \ldots \ldots \ldots \ldots \ldots \ldots \ldots$

Figure 47. Salinity: SF \& NP $\ldots \ldots \ldots \ldots \ldots \ldots \ldots \ldots \ldots \ldots \ldots \ldots \ldots \ldots \ldots$

Figure 48. Light Transmission: SF, CR, ST2 $\ldots \ldots \ldots \ldots \ldots \ldots \ldots \ldots \ldots \ldots \ldots$

Figure 49. ST1 BASS 1 \& SF OBS, NP Bottom Pressure $\ldots \ldots \ldots \ldots \ldots \ldots \ldots$

Figure 50. Fluorometer/Attenuation: SF, CR, \& ST2 $\ldots \ldots \ldots \ldots \ldots \ldots \ldots \ldots$ 


\title{
The 1995 Georges Bank Stratification Study Moored Array Measurements
}

\author{
C. Alessi, R. Beardsley, M. Caruso, J. Churchill, J. Irish, S. Lentz, R. Limeburner, J. Manning, \\ P. Smith, R. Weller, S. Werner, A. Williams, W. Williams
}

\begin{abstract}
The 1995 Georges Bank Stratification Study (GBSS) was the first intensive process study conducted as part of the U.S. GLOBEC Northwest Atlantic/Georges Bank field program. The GBSS was designed to investigate the physical processes which control the seasonal development of stratification along the southern flank of Georges Bank during spring and summer. Past work suggested that during this period, larval cod and haddock tended to aggregate in the thermocline on the southern flank where higher concentrations of their copepod prey were found. A moored array was deployed as part of the GBSS to observe the onset and evolution of seasonal stratification over the southern flank with sufficient vertical and horizontal resolution that key physical processes could be identified and quantified. Moored current, temperature, and conductivity (salinity) measurements were made at three sites on the southern flank, one on the crest, and one on the northeast peak of the bank. Moored surface meteorological measurements were also made at one southern flank site to determine the surface wind stress and heat and moisture fluxes.

The oceanographic and meteorological data collected with the GBSS array during January-August, 1995 are presented in this report. Meteorological data collected on National Data Buoy Center environmental buoys 44011 (Georges Bank), 44008 (Nantucket Shoals), and 44005 (Gulf of Maine) are included in this report for completeness and comparison with the GBSS southern flank meteorological measurements.
\end{abstract}

\section{Introduction}

\subsection{U.S. GLOBEC Northwest Atlantic/Georges Bank Program}

The U.S. GLOBal ocean ECosystem (GLOBEC) Northwest Atlantic/Georges Bank program is a large multi-disciplinary oceanographic research effort funded by the National Science Foundation and the National Marine Fisheries Service (NOAA). The program started in 1993 with intensive field measurements made on Georges Bank and adjacent waters over the six year period 19931999. The primary program objective is to understand the development of zooplankton and larval fish communities on Georges Bank, with special emphasis on the physical and biological processes which influence the population dynamics of four target species: the groundfish cod (Gadus morhua) and haddock (Melanogrammus aeglefinus), and their zooplankton prey, (Calanus finmarchicus and Pseudocalanus) (GLOBEC, 1992). These species were chosen because they are important members of the Georges Bank and other North Atlantic ecosystems, and their populations are likely sensitive to changing climatic conditions. The ultimate program goal is to be able to predict changes in the distribution and abundance of these species as a result of changes in their physical and biotic environment as well as to anticipate how their populations might respond to climate change. (For additional program information, see the U.S. GLOBEC Georges Bank website http://globec.whoi.edu) 


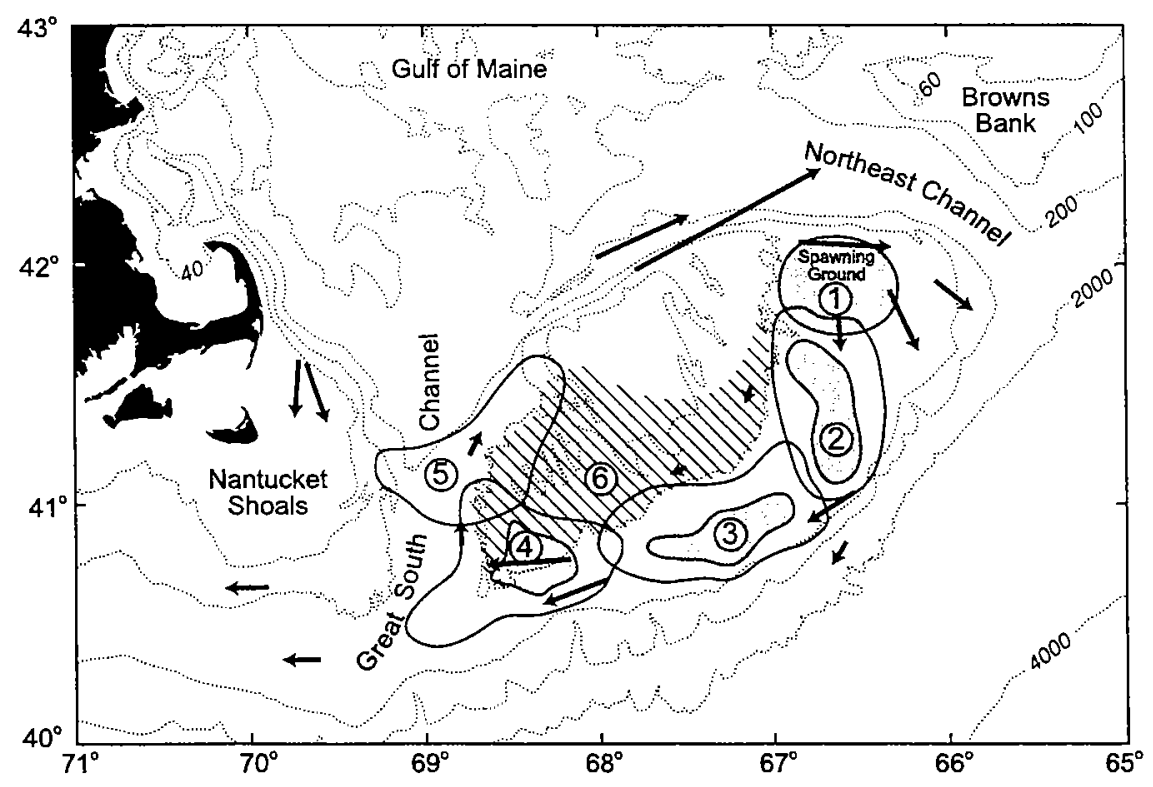

Figure 1: The GLOBEC fish paradigm, showing generalized distribution of cod/haddock eggs (1), larvae $(2,3)$ and pelagic juveniles $(4,5)$ during the first 3-4 months of life in the clockwise circulation over Georges Bank. Cross-hatching indicates where the highest abundance of recentlysettled juveniles (6) may be found in mid-summer. The arrows represent the direction and relative speed of the mean subsurface flows (GLOBEC, 1992).

Cod and haddock generally spawn in winter (January-March) over the northern half of Georges Bank and develop into active swimming juveniles by summer (June-August). The original GLOBEC paradigm for these pelagic early life-history stages (Figure 1) suggests that the physical and biological processes that occur during this January-July period when the mostly passive pre-juvenile fish are advected along the southern flank are most critical to recruitment. For these reasons, the GLOBEC Georges Bank field effort featured a combination of broadscale/long-term sampling and focused process studies. A sequence of broadscale surveys were made during January-May each year during 1995-1999 to measure the populations of the target species over the bank and thus monitor population changes during this critical time period each year. Long-term moored and drifter measurements were also made to allow investigation of potential linkages between population changes and physical events and processes. To examine in detail physical and biological processes thought to be most critical in larval fish development and survival, three process studies were conducted: the Stratification Study (1995) (this report); the Source/Sink Study (1997) and the Cross-Frontal Exchange Study (1999).

\subsection{Georges Bank Stratification Study}

The 1995 Georges Bank Stratification Study (GBSS) was designed to investigate the physical processes which control the seasonal development of stratification along the southern flank of Georges Bank during spring and summer and its influence on the distribution, abundance, health, and behavior of the target species. The vital rates of these animals are strongly dependent on temperature, and some previous work (Buckley and Lough, 1987) suggested that larval cod and 


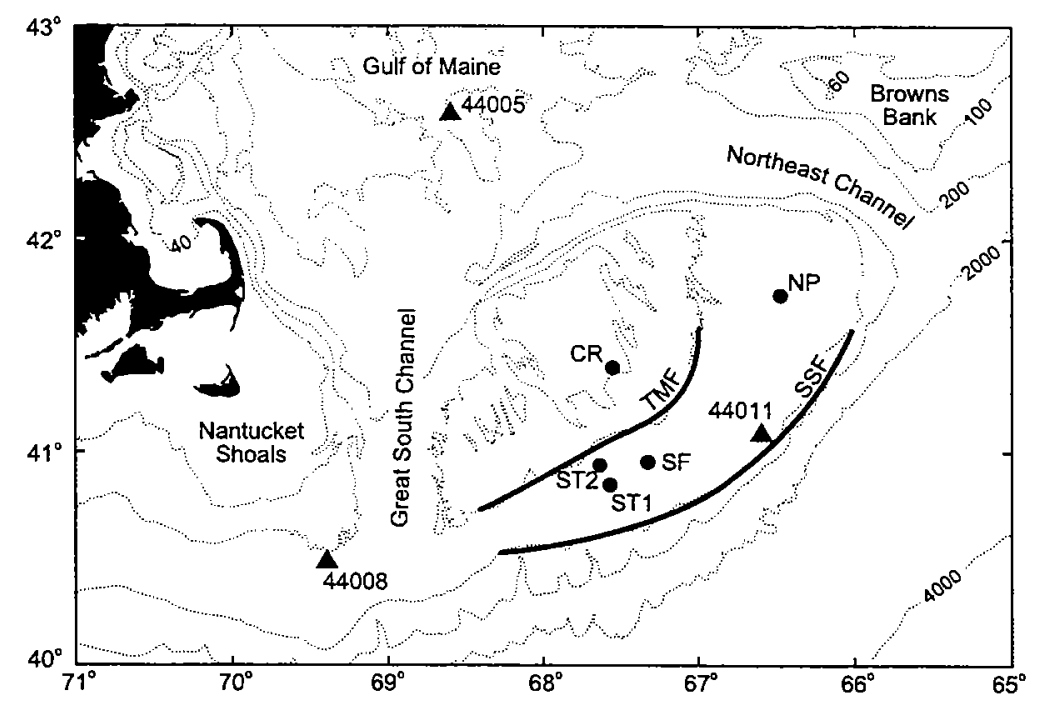

Figure 2: The GBSS moored array, showing the placement of the two process-oriented mooring sites ST1 and ST2, the three long-term mooring sites CR, SF, and NP, and the three NDBC environmental buoys 44011, 44005, and 44008. The approximate positions of the tidal mixing front (TMF) and the shelf/slope front (SSF) along the southern flank are shown. The Northeast Channel (NEC) and Great South Channel (GSC) form the eastern and western boundaries of Georges Bank. Data obtained at the two BIO long-term sites C2 and NECE north of the bank are not included in this report but are available online.

haddock tended to aggregate in the thermocline on the southern flank where higher concentrations of their copepod food were found. For these reasons, moored current, temperature, and conductivity (salinity) measurements were made on the southern flank to observe the onset and evolution of seasonal stratification with sufficient vertical and horizontal resolution that the key processes involved could be identified and quantified. Moored surface meteorological measurements were also made there to determine the surface wind stress and heat flux, so that the influences of surface heating and moisture flux on heat and salt budgets could be examined. These process-oriented moored array measurements were complimented by the long-term moored measurements made at other sites on the bank.

The combined GBSS moored array consisted of the two process-oriented sites (ST1 and ST2) and one long-term site (SF) on the southern flank, the long-term site on the crest (CR), and the long-term site on the Northeast Peak (NP) (Figure 2). Staff at the Woods Hole Oceanographic Institution (WHOI) were responsible for the instrumentation and mooring work at the ST1, SF, and CR sites, while National Marine Fisheries Service (NMFS) staff were responsible for ST2. The NP instrumentation, normally deployed at a site on the western side of the Northeast Channel as part of the Bedford Institute of Oceanography (BIO) long-term array to monitor upstream conditions during the GLOBEC Georges Bank program, were deployed at NP for the 1995 GBSS.

Oceanographic and meteorological data collected with the combined GBSS array during JanuaryAugust, 1995 are presented in this report. Meteorological data collected on National Data Buoy Center (NDBC) environmental buoys 44011 (Georges Bank), 44008 (Nantucket Shoals), and 44005 (Gulf of Maine) are included in this report for completeness and comparison with the meteorological measurements made at ST1. Other physical data collected during the Georges Bank Stratification Study (e.g., moored data collected at the other BIO long-term sites, shipboard 
CTD and ADCP profile data collected on the broadscale, mooring; and process-oriented cruises, ARGOS-tracked drifter trajectories, AVHRR imagery) will not be presented here but are available at http://globec.whoi.edu.

\subsection{Objectives and Organization of this Report}

This report has three primary objectives. The first is to provide a basic description of the GBSS moored array, its design and instrumentation, instrument performance, data return, and the methods used to obtain best edited time series of the data. The second is to present statistical and graphical summaries of the data. The third objective is to provide sufficient information about the 1995 GBSS moored array component that other investigators will be encouraged to obtain this report and data via the GLOBEC Georges Bank website and use them in their own studies. Both hardcopy and online versions of this report are available.

This report is organized as follows:

- Section 2 gives a description of the moored array design, instrumentation, and the placement of instruments at the various measurement sites.

- Section 3 provides a summary table of the moored instrumentation.

- Section 4 gives a detailed discussion of the methods used to produce the best edited final data records, the associated data return, and problems affecting the data return.

- Section 5 describes how to access the data via the Internet.

- Section 6 lists the GLOBEC cruise reports related to the GBSS moored array.

- Section 7 presents basic statistics of the hourly-averaged data.

- Section 8 presents time series plots for each record.

\section{Stratification Study Moored Array}

The combined GBSS moored array included the two process-oriented mooring sites on the southern flank (ST1 and ST2), the long-term mooring sites on the southern flank (SF), crest (CR) and Northeast Peak (NP), plus the three nearest NDBC environmental buoys (Figure 2). The ST1 and ST2 moorings were deployed in late January 1995 when the water column was generally well mixed vertically and recovered in early August near the maximum in seasonal stratification. Two shorter deployments of a Benthic Acoustic Stress Sensor (BASS) bottom tripod were made at ST1 to obtain current measurements close to the seafloor. The two WHOI long-term moorings at SF and CR were first deployed in November 1994, serviced in spring and recovered in October 1995. The BIO long-term moorings at NP were deployed in June 1994 and recovered the following June. The mooring positions and water depths at these sites are given in Table 1, and a deployment timeline shown in Figure 3. 
Table 1: Summary of instrumented mooring positions and depths for the combined GBSS moored array. The positions were determined by a combination of differential GPS, standard GPS, radar and visual relative orientation. The positions of the uninstrumented guard buoys are not included.

\begin{tabular}{|c|c|c|c|c|c|}
\hline $\begin{array}{c}\text { Station } \\
\text { Name }\end{array}$ & $\begin{array}{l}\text { Data } \\
\text { Source }\end{array}$ & $\begin{array}{l}\text { Abbre- } \\
\text { viation }\end{array}$ & $\begin{array}{l}\text { Water } \\
\text { Depth }\end{array}$ & \multicolumn{2}{|c|}{$\begin{array}{l}\text { Location } \\
\left({ }^{\circ} \mathrm{N} /{ }^{\circ} \mathrm{W}\right)\end{array}$} \\
\hline \multicolumn{6}{|l|}{$\underline{\mathrm{ST} 1}$} \\
\hline Surface & WHOI & ST1 & $76 \mathrm{~m}$ & $40^{\circ} 51.85^{\prime}$ & $67^{\circ} 33.53^{\prime}$ \\
\hline Subsurface & WHOI & ST1 & $76 \mathrm{~m}$ & $40^{\circ} 51.72^{\prime}$ & $67^{\circ} 33.61^{\prime}$ \\
\hline BASS Tripod & WHOI & ST1B & $77 \mathrm{~m}$ & $40^{\circ} 51.67^{\prime}$ & $67^{\circ} 33.59^{\prime}$ \\
\hline \multicolumn{6}{|l|}{$\underline{\text { ST2 }}$} \\
\hline Surface & NMFS & $\mathrm{ST} 2$ & $68 \mathrm{~m}$ & $40^{\circ} 57.35^{\prime}$ & $67^{\circ} 37.59^{\prime}$ \\
\hline \multicolumn{6}{|l|}{ Southern Flank } \\
\hline Surface & WHOI & SF & $75 \mathrm{~m}$ & $40^{\circ} 58.15^{\prime}$ & $67^{\circ} 19.14^{\prime}$ \\
\hline \multicolumn{6}{|l|}{$\underline{\text { Crest }}$} \\
\hline Surface & WHOI & $\mathrm{CR}$ & $43 \mathrm{~m}$ & $40^{\circ} 57.35^{\prime}$ & $67^{\circ} 37.59^{\prime}$ \\
\hline \multicolumn{6}{|l|}{ Northeast Peak } \\
\hline Subsurface 1 & $\mathrm{BIO}$ & NP & $73 \mathrm{~m}$ & $41^{\circ} 44.01^{\prime}$ & $66^{\circ} 32.08^{\prime}$ \\
\hline Subsurface 2 & $\mathrm{BIO}$ & NP & $73 \mathrm{~m}$ & $41^{\circ} 44.01^{\prime}$ & $66^{\circ} 32.30^{\prime}$ \\
\hline \multicolumn{6}{|c|}{ NDBC Environmental Buoys } \\
\hline Gulf of Maine & NDBC & 44005 & $29 \mathrm{~m}$ & $42^{\circ} 53.54^{\prime}$ & $68^{\circ} 56.38^{\prime}$ \\
\hline Nantucket Shoals & $\mathrm{NDBC}$ & 44008 & $55 \mathrm{~m}$ & $40^{\circ} 30.01^{\prime}$ & $69^{\circ} 25.54^{\prime}$ \\
\hline Georges Bank & $\mathrm{NDBC}$ & 44011 & $88 \mathrm{~m}$ & $41^{\circ} 04.80^{\prime}$ & $66^{\circ} 34.80^{\prime}$ \\
\hline
\end{tabular}


Time Period of GLOBEC Moored Array and Hydrographic Components

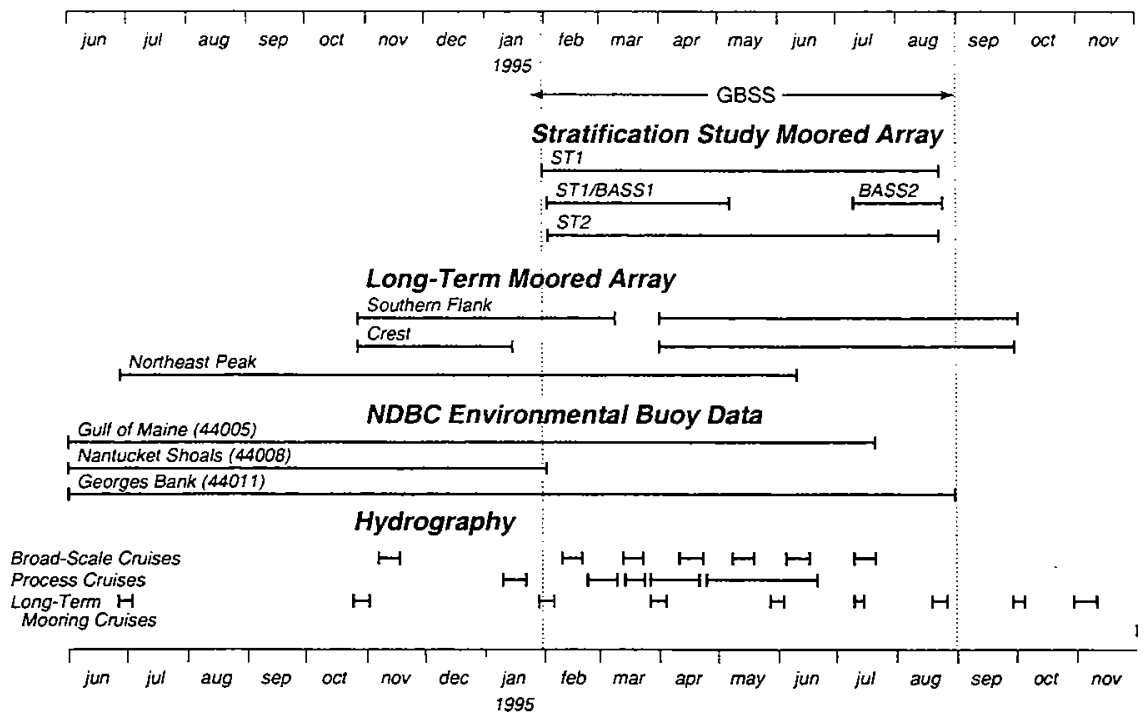

Figure 3: Timeline for GBSS moored array, showing when different mooring sites were instrumented. The period January 31 - August 31, 1995 presented in this report is indicated. Also shown is when the NDBC environmental buoys returned useful data.

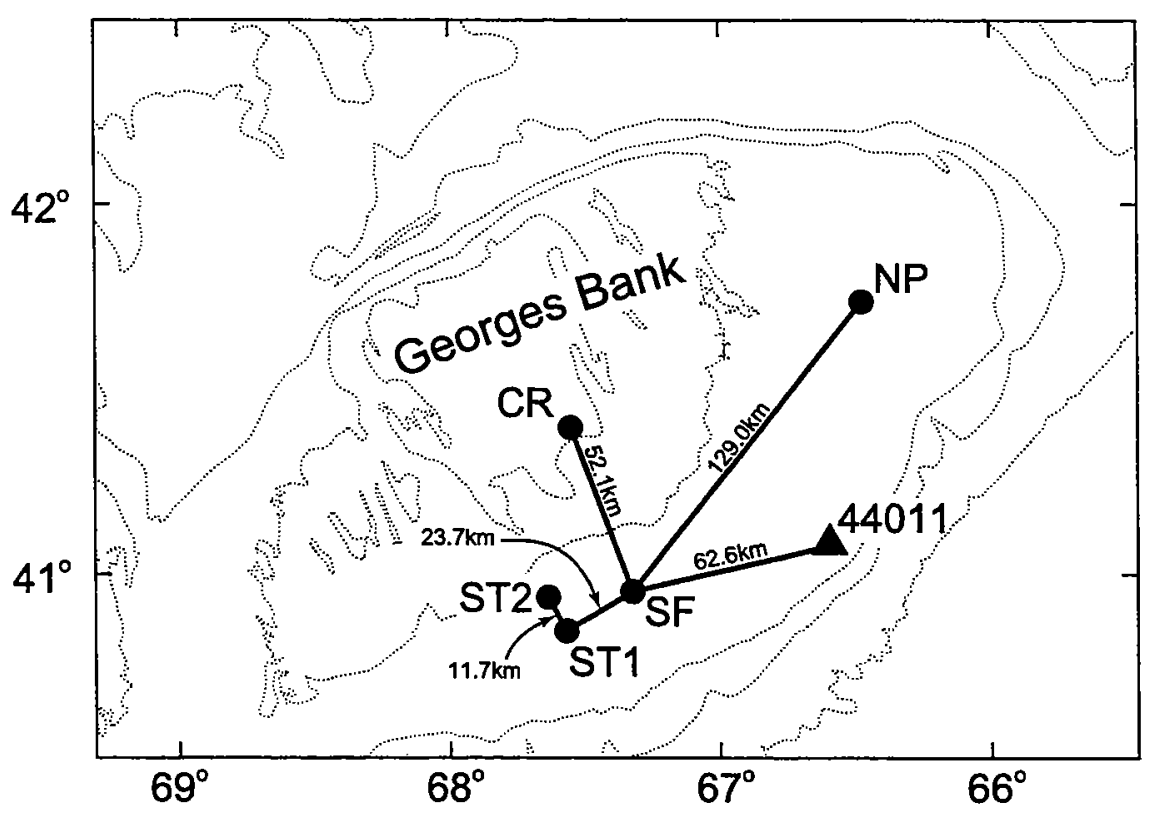

Figure 4: GBSS Mooring Separation 


\subsection{Moored Array Design}

The long-term southern flank mooring site SF was chosen to lie in the strongly stratified zone between the tidal mixing front that forms near the $60-\mathrm{m}$ isobath during the stratified season and the shelf/slope front that separates shelf water from the more saline slope water (Figure 2). The base of the shelf/slope front nominally lies near the $\sim 90-100 \mathrm{~m}$ isobath along the southern flank of Georges Bank, so that principally shelf (and not slope) water would occupy the bottom at SF except possibly during strong slope water intrusions. The ST1 site was placed about $23.5 \mathrm{~km}$ to the southwest of SF along about the same isobath $(76-\mathrm{m})$ so that meaningful estimates of alongisobath gradients could be made between ST1 and SF (Figure 4). The ST2 mooring site was then located $11.7 \mathrm{~km}$ onbank of ST1 in water $67 \mathrm{~m}$ deep, just offbank of the tidal mixing front, to allow meaningful estimation of cross-isobath current and stratification gradients between ST1 and ST2.

\section{$2.2 \mathrm{ST} 1$}

The WHOI moored array at ST1 consisted of a surface 3-m discus buoy with meteorological instrumentation supporting eight Vector-Measuring Current Meters (VMCMs) and other subsurface instrumentation down to a depth of $40 \mathrm{~m}$, a subsurface mooring supporting five VMCMs and other instrumentation over the lower $30 \mathrm{~m}$ of the water column, the BASS tripod, and three uninstrumented surface guard moorings.

The surface moorings (the discus and three guard moorings) were deployed in a tight square pattern to protect the two subsurface moorings placed within the square. The final mooring positions shown in Figure 5 and Table 1 are based on a subjective combination of differential GPS positions at time of anchor drop, radar ranges, visual bearings, and relative orientations. The water depths listed for each mooring were taken from the ship's PDR at time of anchor drop or release of the BASS tripod. No correction for tides $(<1 \mathrm{~m})$ was made. Analysis of data from the Edo depth sounder that was recorded with the GPS position data during the deployment cruise suggests that the water depth varied by 1-2 m over the 300-m extent of the ST1 array.

The ST1 discus buoy supported two independent meteorological packages, a Vector Averaging Wind Recorder (VAWR) (Dean and Beardsley, 1988) and a Improved Meteorological Instrumentation (IMET) package (Weller et al., 1990; Hosom et al., 1995) (Figure 6). Each system measured wind speed and direction, air and sea surface temperatures, relative humidity and incident short- and long-wave radiation, while the IMET also recorded precipitation. The VAWR recorded data every 15 minutes while the IMET used a 1-minute record rate. A steering vane was used to orient the discus buoy so that the wind, air temperature/relative humidity, and air pressure sensors were heading into the wind. These redundant sets of sensors with separate loggers were deployed to help ensure that the measurements needed to estimate air-sea heat fluxes, surface stress, and buoyancy input would be obtained. The VAWR and IMET specifications are listed in Tables 2 and 3, and the sensor heights above the mean buoy water line are given in Table 4 . 
Table 2: WHOI VAWR Specifications

\begin{tabular}{|c|c|c|c|}
\hline Parameter & Sensor Type & Accuracy & Record Time \\
\hline Wind Speed & $\begin{array}{c}\text { Three-Cup Anemometer } \\
\text { R. M. Young }\end{array}$ & $\begin{array}{l} \pm 2 \% \text { above } \\
0.7 \mathrm{~m} / \mathrm{s}\end{array}$ & $\begin{array}{l}\text { 900-Sec } \\
\text { Vector Average }\end{array}$ \\
\hline Wind Direction & $\begin{array}{l}\text { Integral Vane } \\
\text { w/ Vane Follower } \\
\text { WHOI/EG\&G }\end{array}$ & $\pm 5.6^{\circ}$ & 900-Sec Average \\
\hline Air Temperature & $\begin{array}{l}\text { Thermistor } \\
\text { Yellow Springs } \\
5 \mathrm{~K} @ 25^{\circ} \mathrm{C}\end{array}$ & $\begin{array}{l} \pm 0.2^{\circ} \mathrm{C} \text { when } \\
\text { Wind }>5 \mathrm{~m} / \mathrm{s}\end{array}$ & $\begin{array}{c}\text { 450-Sec Average } \\
\text { (Half-Record Time) } \\
\text { (Note 1) }\end{array}$ \\
\hline Relative Humidity & $\begin{array}{l}\text { Vaisala Humicap } \\
\text { 0062HMP }\end{array}$ & $\pm 2 \% \mathrm{RH}$ & $\begin{array}{c}\text { 3.515-Sec Average } \\
\text { (Note 2) }\end{array}$ \\
\hline Barometric Pressure & $\begin{array}{l}\text { Model 216-B-101 } \\
\text { Paroscientific }\end{array}$ & $\begin{array}{c} \pm 0.2 \text { mbars when } \\
\text { Wind }<20 \mathrm{~m} / \mathrm{s}\end{array}$ & $\begin{array}{l}\text { 2.636-Sec Average } \\
\text { (Note 2) }\end{array}$ \\
\hline Short-Wave Radiation & $\begin{array}{l}\text { Pyranometer } \\
\text { Eppley } 8-48\end{array}$ & $\pm 3 \%$ & 900-Sec Average \\
\hline Long-Wave Radiation & $\begin{array}{l}\text { Pyrgeometer } \\
\text { Eppley PIR }\end{array}$ & $\pm 10 \%$ & 900-Sec Average \\
\hline Sea Temperature & $\begin{array}{l}\text { Thermistor } \\
\text { Thermometrics } \\
4 \mathrm{~K} @ 25^{\circ} \mathrm{C}\end{array}$ & $\pm 0.005^{\circ} \mathrm{C}$ & $\begin{array}{c}\text { 450-Sec Average } \\
\text { (Half-Record Time) } \\
\text { (Note 3) }\end{array}$ \\
\hline
\end{tabular}

\section{Notes for the VAWR Specifications}

- Note 1: Air Temperature is measured during the second half of the record interval.

- Note 2: Relative Humidity and Barometric Pressure are averaged in the middle of the 900-Sec interval for the time noted.

- Note 3: Sea Temperature is measured during the first half of the record interval. 


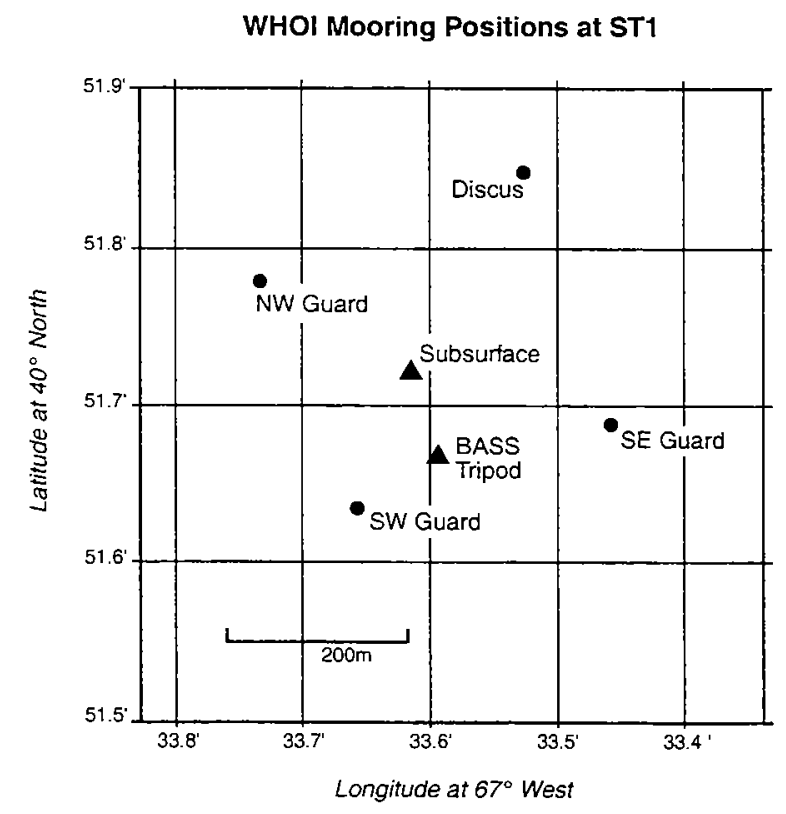

Figure 5: Map of ST1 Mooring Positions

Table 3: WHOI IMET Specifications

\begin{tabular}{|c|c|c|c|}
\hline Parameter & Sensor Type & Accuracy & Record Time \\
\hline Wind Speed & $\begin{array}{l}\text { Modified R.M. Young } \\
\text { Model No. } 05103 \\
\text { Wind Monitor }\end{array}$ & $\pm 2 \%$ & $\begin{array}{l}\text { Vector Average } \\
60-\mathrm{Sec}\end{array}$ \\
\hline Wind Direction & $\begin{array}{c}\text { Modified R. M. Young } \\
\text { Model No. } 05103 \\
\text { Wind Monitor }\end{array}$ & $\pm 3^{\circ}$ & $\begin{array}{l}\text { Vector Average } \\
60-\mathrm{Sec}\end{array}$ \\
\hline Air Temperature & $\begin{array}{c}\text { Rotronic } \\
\text { Model MP-101A }\end{array}$ & $\begin{array}{c} \pm 0.3^{\circ} \mathrm{C} \text { for } \\
\text { Wind }>5 \mathrm{~m} / \mathrm{s}\end{array}$ & 60-Sec Average \\
\hline Relative Humidity & $\begin{array}{c}\text { Rotronic } \\
\text { Model MP-101A }\end{array}$ & $\pm 2 \% \mathrm{RH}$ & 3.515-Sec Average \\
\hline Barometric Pressure & $\begin{array}{c}\text { Paroscientific } \\
\text { Model 52-B }\end{array}$ & $\begin{array}{c} \pm 0.5 \text { mbars when } \\
\text { Wind }<20 \mathrm{~m} / \mathrm{s}\end{array}$ & 2.636-Sec Average \\
\hline Shortwave Radiation & $\begin{array}{l}\text { Pyranometer } \\
\text { Eppley PSP }\end{array}$ & $\pm 3 \%$ & 60-Sec Average \\
\hline Long-Wave Radiation & $\begin{array}{l}\text { Pyrgeometer } \\
\text { Eppley PIR }\end{array}$ & $10 \mathrm{~W} / \mathrm{m}^{2}$ & 60-Sec Average \\
\hline Sea Temperature & PRT & $\pm 0.005^{\circ} \mathrm{C}$ & 60-Sec Average \\
\hline Precipitation & $\begin{array}{l}\text { R.M. Young } \\
\text { Rain Gauge }\end{array}$ & $\pm 10 \% \mathrm{C}$ & 60-Sec Average \\
\hline
\end{tabular}




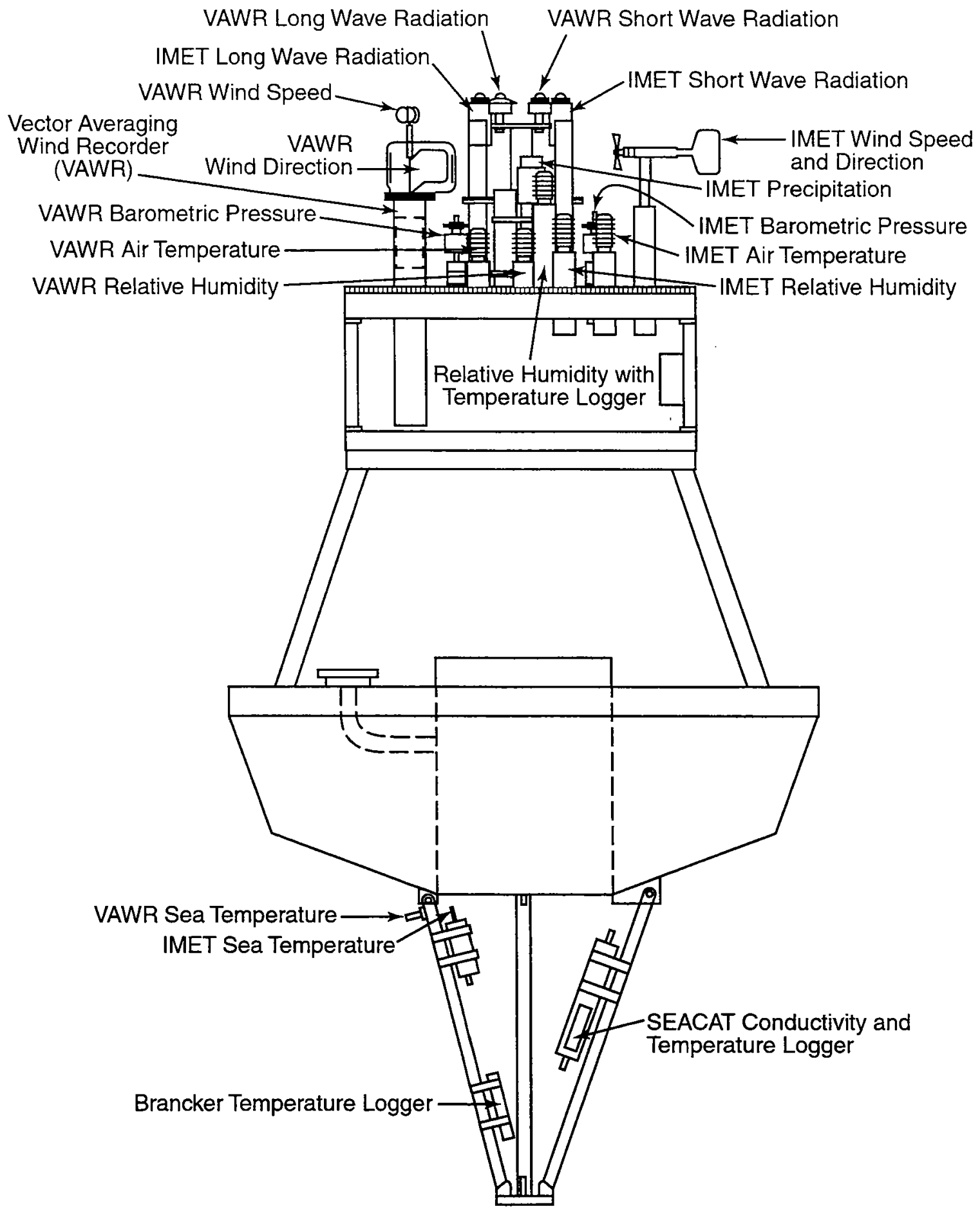

Figure 6A: Schematic of 3-m discus buoy with VAWR and IMET Instrumentation: (A) Side-view; A steering vane was mounted on one side of the discus tower. This vane kept the buoy and its sensors pointing into the wind. The vane is located on the tower apex opposite the sensors, and is not shown in this schematic. (B) Top view of discus tower (see next figure). 


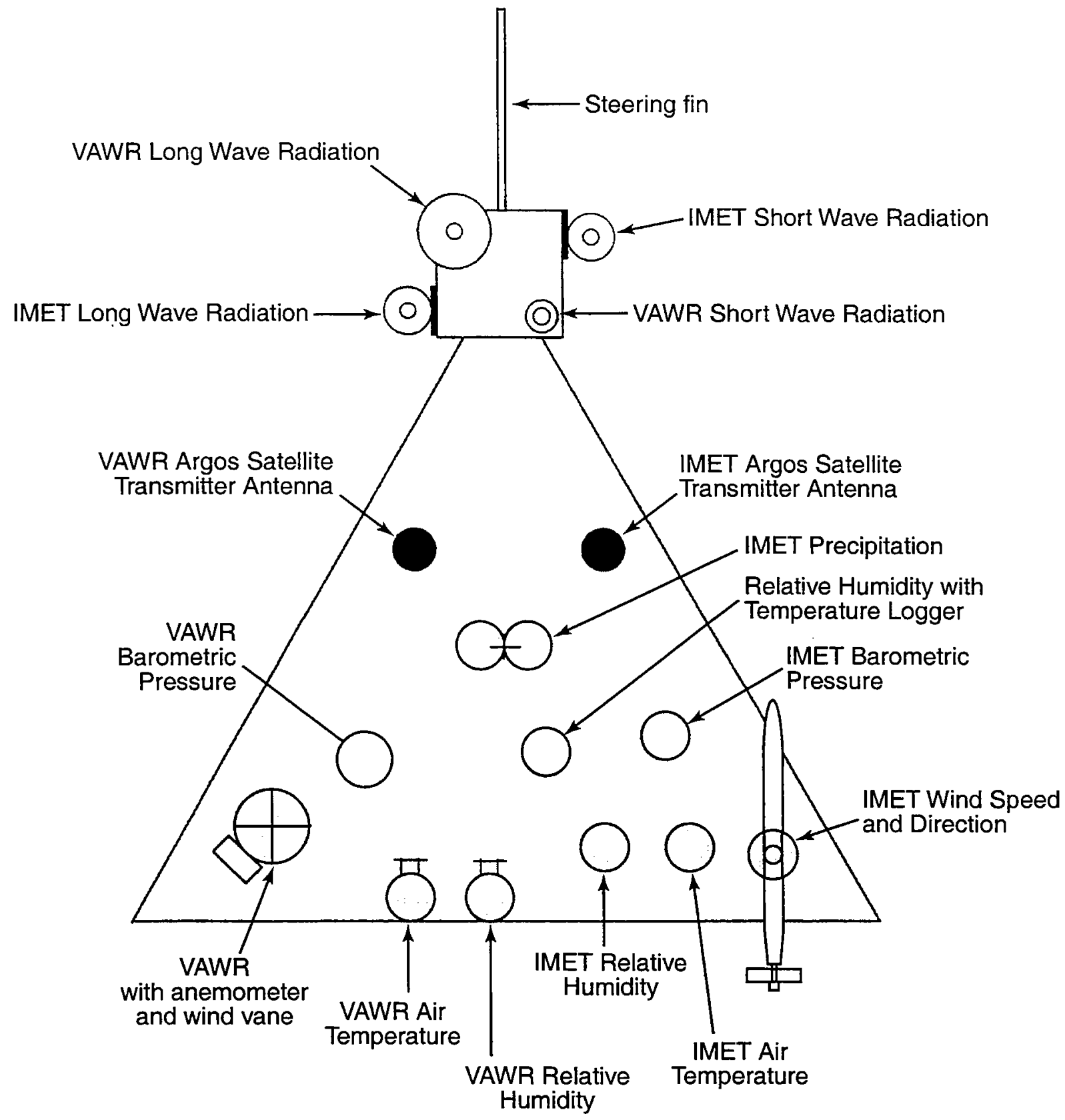

Figure 6B: Top view of discus tower. (Schematic of 3-m discus buoy with VAWR and IMET Instrumentation. See caption for $6 \mathrm{~A}$.) 
Table 4: VAWR and IMET Sensor Heights

\begin{tabular}{|l|c|c|}
\hline \multicolumn{1}{|c|}{ Parameter } & VAWR* & IMET $^{*}$ \\
\hline Wind Speed and Direction & 3.33 & 3.23 \\
Short-Wave Radiation & 3.40 & 3.41 \\
Long-Wave Radiation & 3.42 & 3.45 \\
Atmospheric Pressure & 2.74 & 2.80 \\
Relative Humidity & 2.68 & 2.74 \\
Air Temperature & 2.67 & 2.74 \\
Rainfall & - & 3.10 \\
Sea Surface Temperature & -1.00 & -1.00 \\
\hline
\end{tabular}

*Heights are in meters above and below $(-)$ the water line.

Despite a series of storms with winds in excess of $18 \mathrm{~m} / \mathrm{s}$ and short, steep waves which battered the discus buoy, the moorings deployed at ST1 remained on station throughout the experiment. However, daily monitoring via ARGOS indicated that one long-wave sensor failed on March 1 and the IMET and VAWR wind sensors failed on May 16 and June 3, respectively. The long-wave sensor was replaced on June 11, and both IMET and VAWR wind sensors were replaced on July 11. Comparison of the ST1 wind data with the Georges Bank NDBC buoy 41011 winds (discussed in Section 4) indicates that the ST1 missing wind data could be estimated (to within $\pm 1.4 \mathrm{~m} / \mathrm{s}$ ) from buoy 44011 data (as discussed later in this report). With these exceptions, the other ST1 meteorological sensors returned complete records.

The surface and subsurface moorings at ST1 supported 13 VMCMs and six Sea-Bird SeaCat temperature and conductivity recorders (Figures 7 and 8). Self-contained Brancker temperature recorders (TPODS) were placed at nine additional depths. The VMCMs recorded data every $7.5 \mathrm{~min}$, the SeaCats every $1.5 \mathrm{~min}$, and the Brancker TPODS every 30 seconds (Tables 5, 6, and 7). The ST1 oceanographic instruments returned full records with the exception of no return from three TPODs and two of the deeper VMCMs.

Table 5: WHOI VMCM Specifications

\begin{tabular}{|l|l|c|c|}
\hline Parameter & Sensor Type & Accuracy & Record Time \\
\hline Speed & Dual Propellers & $\pm 1 \%$ & Averaged Over \\
Direction & Flux-Gate Compass & $\pm 5^{\circ}$ & Full Recording \\
Temperature & Thermistor & $\pm 0.01^{\circ} \mathrm{C}$ & Interval \\
\hline
\end{tabular}


Table 6: Sea-Bird SeaCat Specifications

\begin{tabular}{|c|c|c|c|}
\hline Parameter & Sensor Type & Accuracy & Record Time \\
\hline Temperature & Thermistor & $\pm 0.01^{\circ} \mathrm{C} / 6 \mathrm{mo}$ & $\begin{array}{l}0.125 \text { Sec Sample at } \\
\text { End of Record Cycle }\end{array}$ \\
\hline Conductivity & Four-Electrode Cell & $\pm 0.001 \mathrm{~S} / \mathrm{m} / \mathrm{mo}$ & $\begin{array}{l}0.125 \text { Sec Sample at } \\
\text { End of Record Cycle }\end{array}$ \\
\hline
\end{tabular}

Table 7: Brancker TPOD Specifications

\begin{tabular}{|l|l|l|l|}
\hline Parameter & Sensor Type & Accuracy & Record Time \\
\hline Temperature & Thermistor & $\pm 0.02^{\circ} \mathrm{C}$ & Sample at Time Recorded \\
\hline
\end{tabular}

The BASS bottom tripod at ST1 supported a vertical array of five acoustic travel-time current meters positions from 0.3 to $4.5 \mathrm{~m}$ above the bottom. Optical Backscatter Sensors (OBSs) set at the same height monitored the suspended particulate distribution. The tripod also supported eight temperature sensors distributed from 0.3 to $5.0 \mathrm{~m}$ above the bottom and a bottom pressure recorder.

The BASS current sensors sampled and recorded data at $2 \mathrm{~Hz}$ over one 7.5-minute interval each half hour (Williams et al., 1987). Due to this high sample rate and limited data storage capacity, the BASS tripod was deployed twice at ST1 during the GBSS. BASS1 was deployed February 1 and recovered in June using a ROV after two earlier attempts failed when the recovery line either did not surface or was irretrievably snagged on the nearby guard mooring ground line. BASS1 also carried a camera, strobe light, and battery case to photograph the bottom every eight hours to monitor bottom bedform structure and variability. A schematic of BASS1 is shown in Figure 9. BASS2 was deployed July 10 and recovered August 23. The BASS1 and BASS2 measurement periods and sensor heights above the bottom are given in Table 8 .

Table 8: WHOI BASS Measurement Periods and Sensor Heights

\begin{tabular}{|c|l|l|l|l|}
\hline & \multicolumn{2}{|c|}{ BASS 1 } & \multicolumn{2}{c|}{ BASS 2 } \\
\cline { 2 - 5 } & \multicolumn{1}{|c|}{ Velocity } & \multicolumn{1}{|c|}{ Temperature } & \multicolumn{1}{c|}{ Velocity } & Temperature \\
\hline $\begin{array}{c}\text { Measurement } \\
\text { Period }\end{array}$ & Feb 3-Apr 25 & Feb 3-Apr 25 & Jul 11-Aug 14 & Jul 11-Aug 24 \\
\hline $\begin{array}{c}\text { Sensor Height } \\
\text { (Meters Above } \\
\text { Bottom) }\end{array}$ & $0.22,0.58,1.18$, & $\begin{array}{l}(0.24)^{*}, 0.62,1.22, \\
1.90,2.53,3.24, \\
4.43,5.72\end{array}$ & $0.26,0.61,1.21$, & $\begin{array}{l}0.32,0.58,1.18, \\
1.86,4.46\end{array}$ \\
\hline
\end{tabular}

*No Data Returned. 


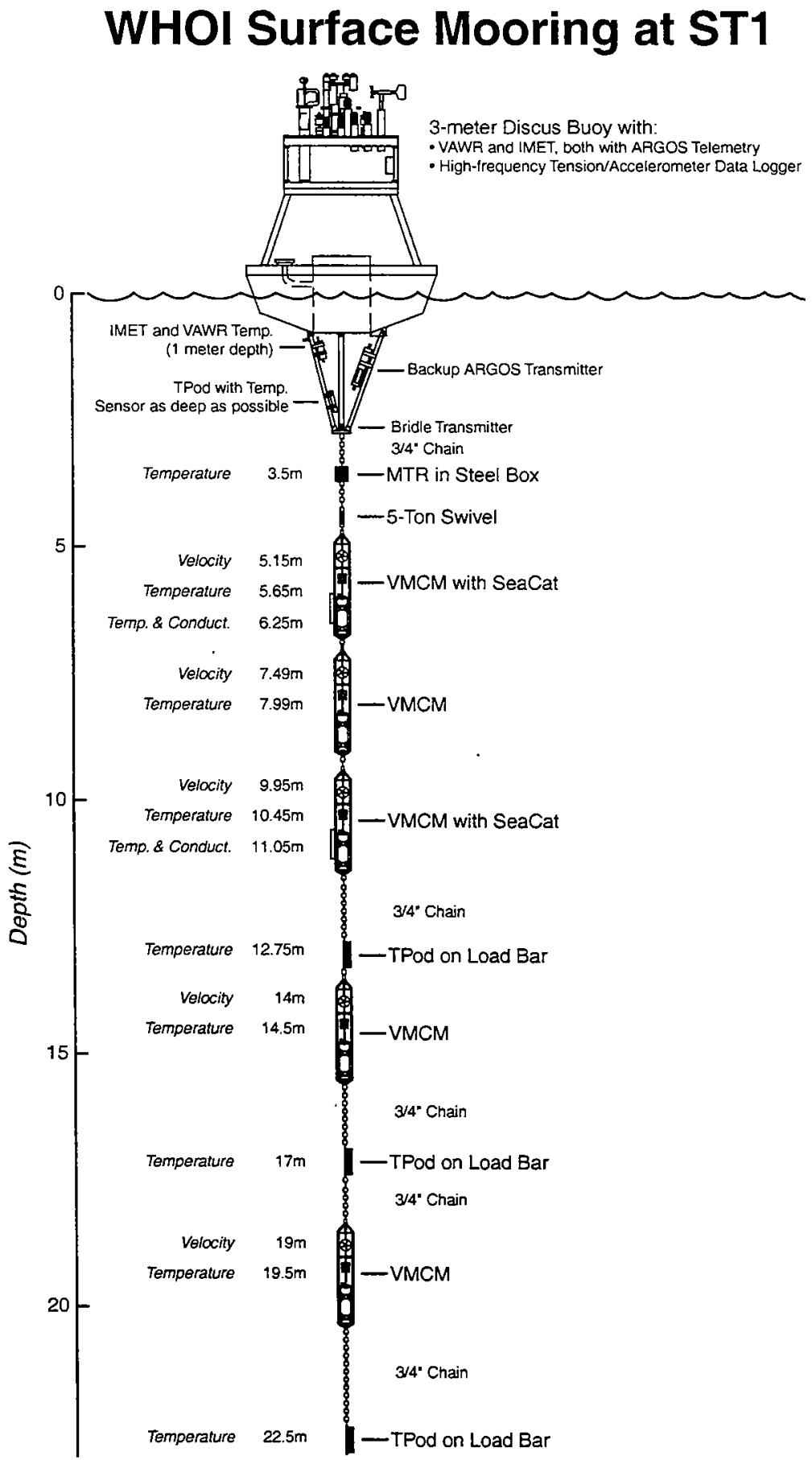

Figure 7A: ST1 Surface Mooring Diagram: (A) upper section ; (B) and (C) mid- and lower sections (see next two figures). 


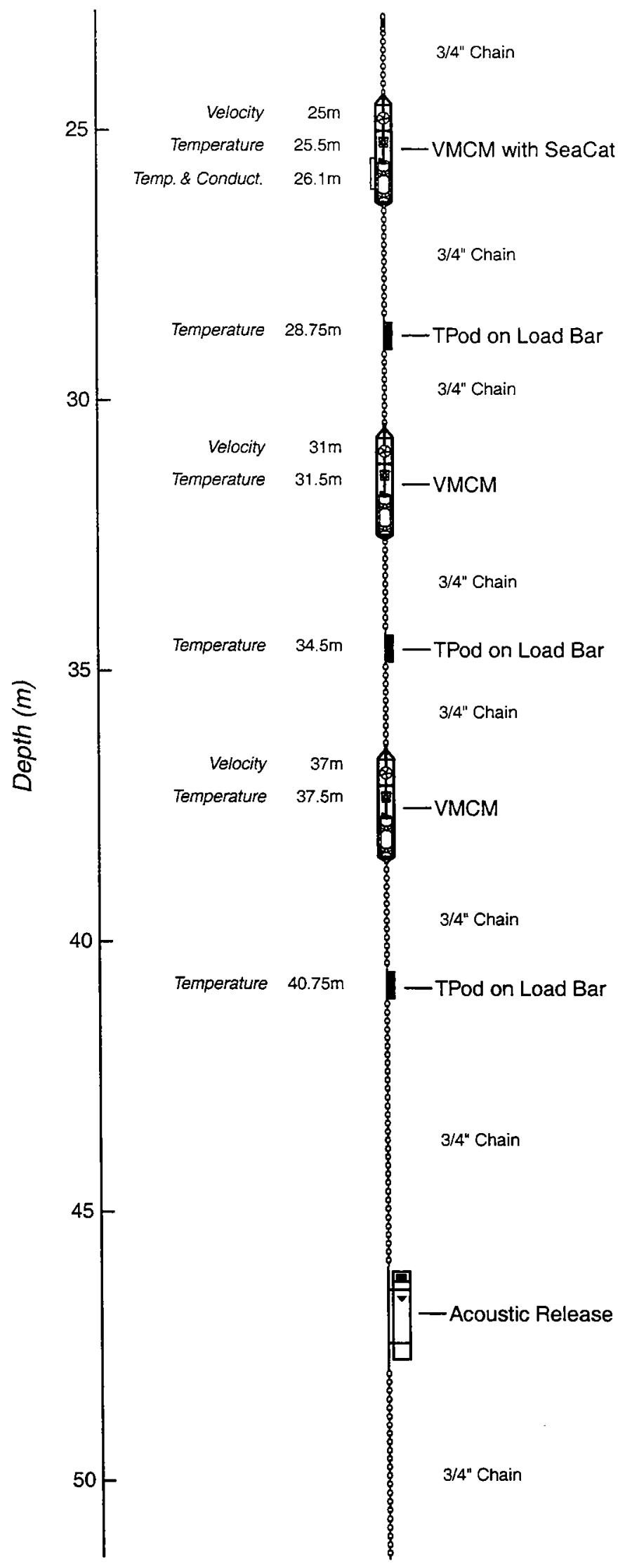

Figure 7B: Mid-section of surface mooring. 


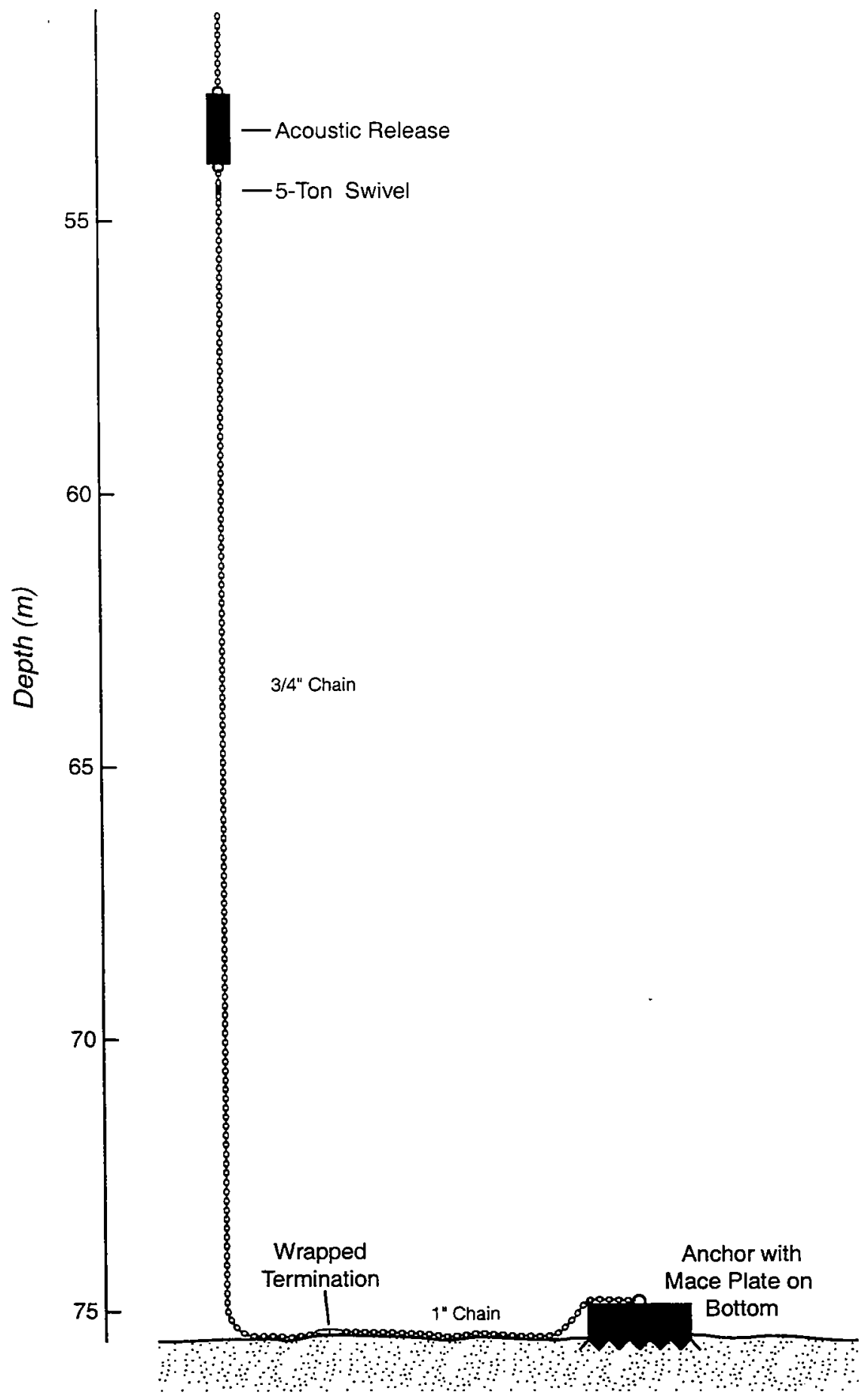

Figure 7C: Lower section of surface mooring. 


\section{WHOI Subsurface Mooring at ST1}

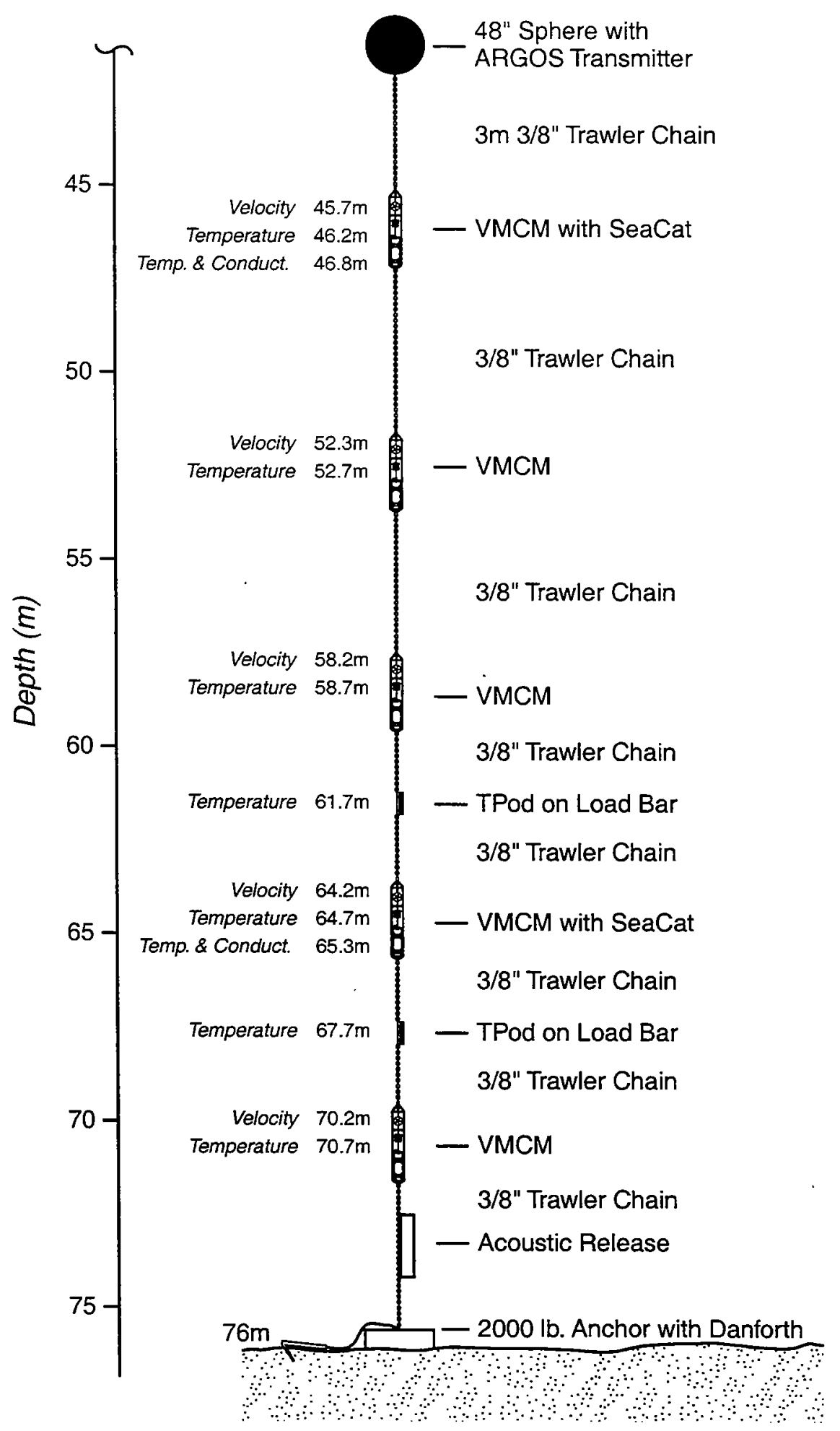

Figure 8: ST1 Subsurface Mooring Diagram 


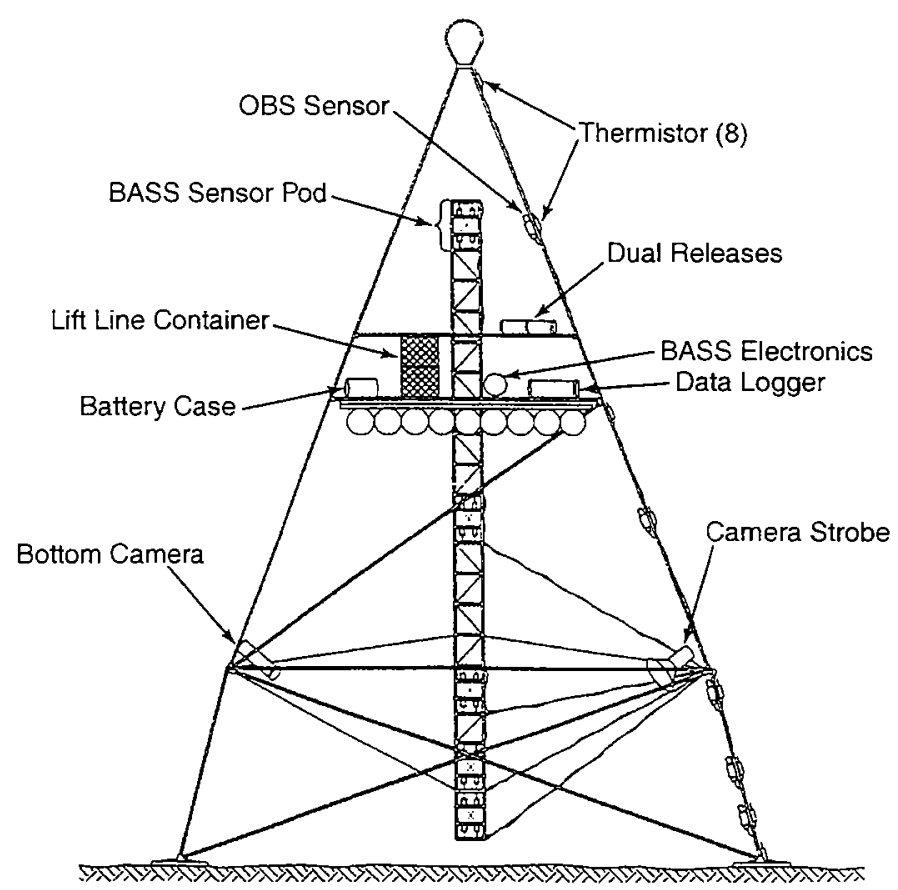

Figure 9: Schematic of BASS1 deployed at ST1 during late winter. The camera, light strobe, and battery case were not mounted on the summer tripod BASS2.

\section{$2.3 \mathrm{ST} 2$}

The NMFS deployed at ST2 a single surface buoy supporting two VMCMs, a near-bottom Vector Averaging Current Meter (VACM) with added conductivity and light transmission sensors (Table 9), four model 16 SeaCats, three Brancker XL-105 TPODs, and one VEMCO MiniLog-TX/TR temperature recorder (Figure 10). The VMCMs, VACM, and SeaCats were set to record every $7.5 \mathrm{~min}$, the TPODS every $30 \mathrm{~min}$, and the Minilog every $60 \mathrm{~min}$. This mooring used an elastic (six-bungy-cord) member to maintain tension on the near-bottom element of the mooring. Its position and water depth listed in Table 1 were taken at time of the anchor drop.

After surviving several months of winter/spring conditions, the upper portion of the mooring was evidently severed by a passing trawler on July 27 th, a few weeks prior to the scheduled recovery. The upper portion of the mooring drifted for two months until a fisherman recovered it off the Hudson Canyon approximately 250 miles away. Consequently, all data was recovered except for one SeaCat. The lower portion of the mooring was acoustically released as scheduled in mid-August from the R/V Endeavor.

\section{$2.4 \mathrm{SF}$}

The WHOI long-term moored array at SF included a central science surface mooring, an instrumented bottom package, one instrumented surface guard buoy (spring and summer only), and one uninstrumented surface guard buoy. The two guard buoys were moored along the 76-m isobath and the science mooring and bottom package between to help protect the instrumentation from 


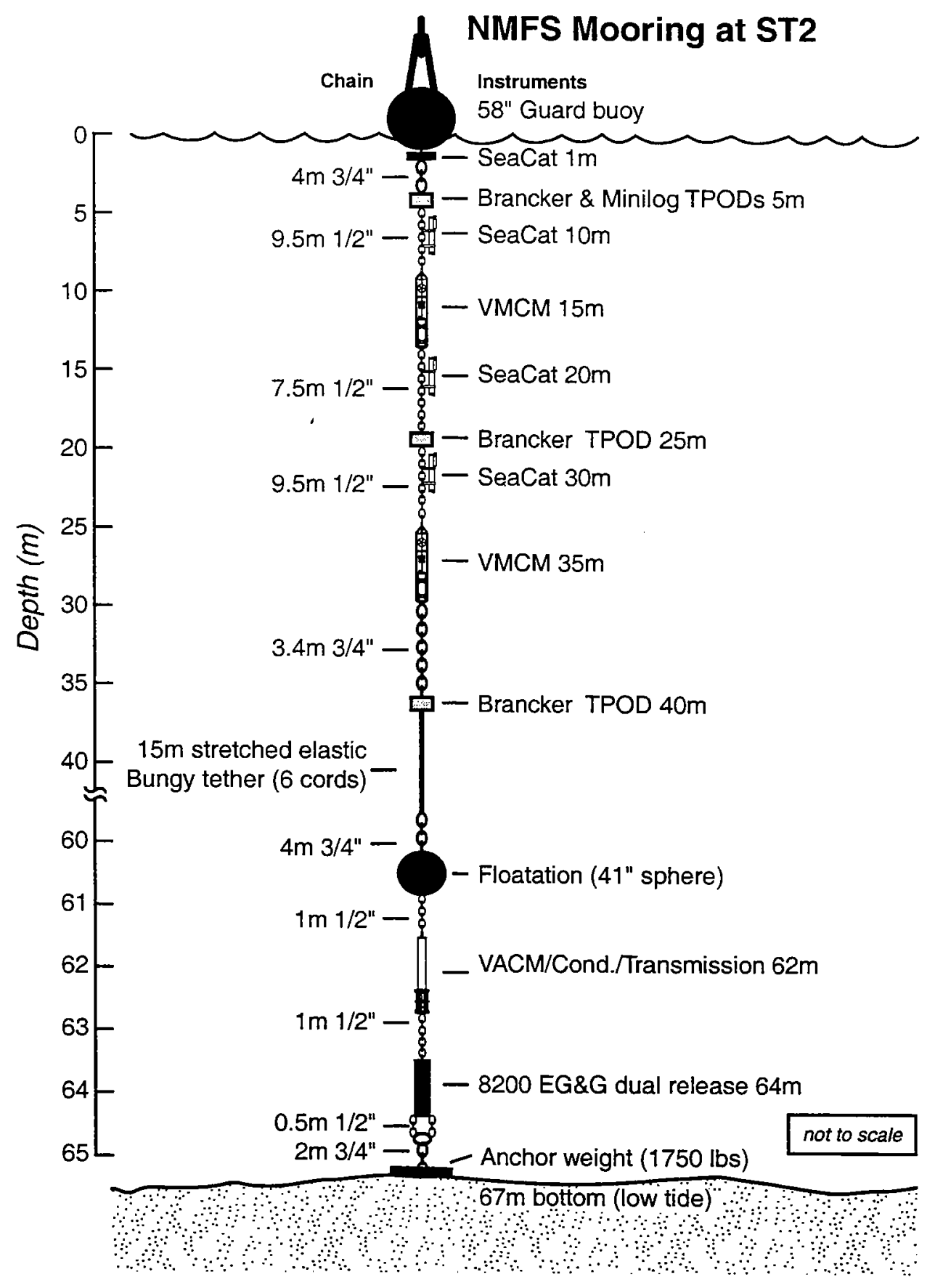

Figure 10: ST2 Mooring Diagram 


\section{WHOI Long-term Mooring at SF}

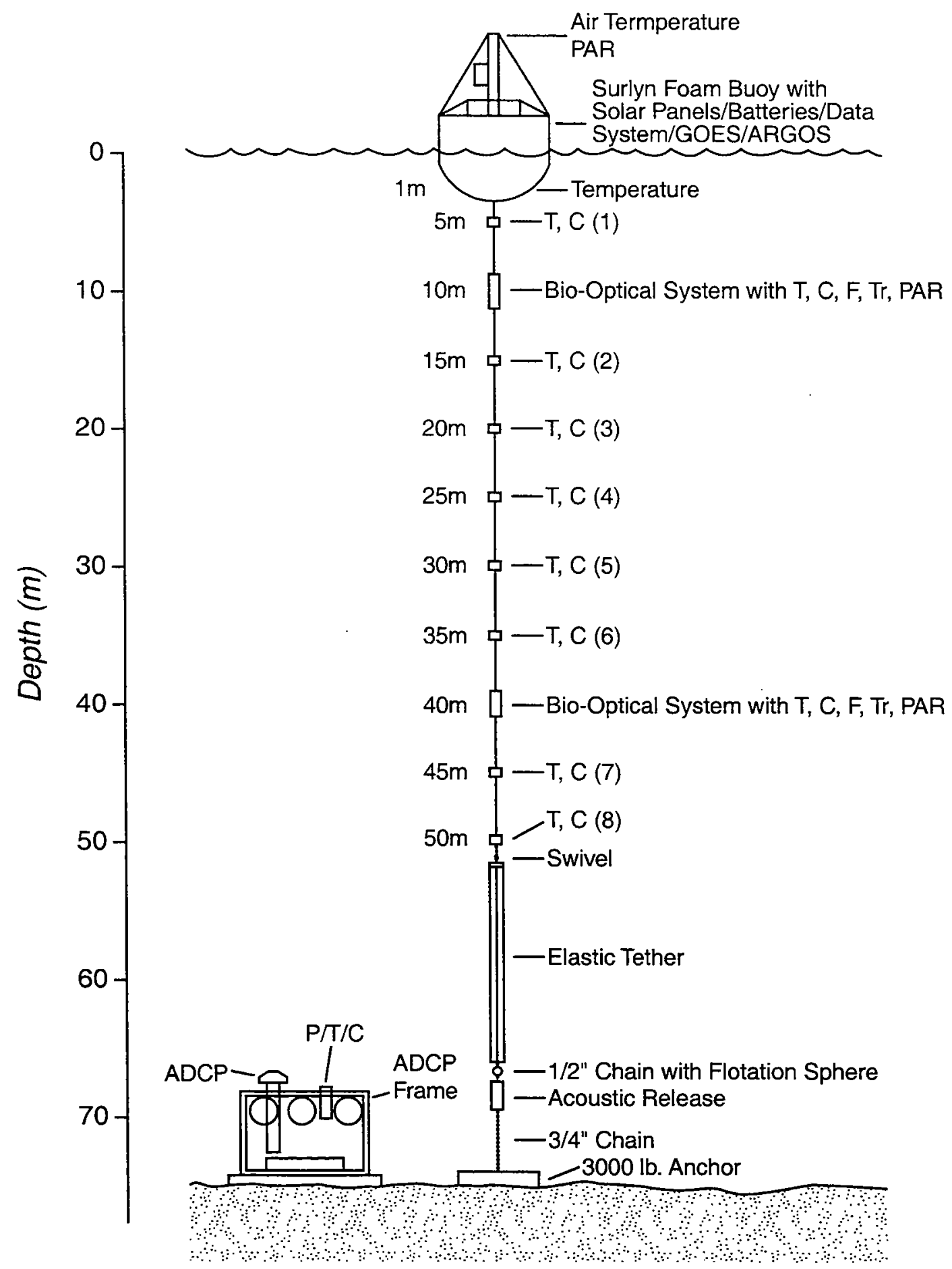

Figure 11: SF Mooring Diagrams 
fishing activities. The central science mooring supported surface sensors for incoming PAR (cosine collector of downwelling photosynthetically active radiation integrated from 400 to $700 \mathrm{~nm}$ ), and air and sea surface temperature. A vertical chain of eight pairs of Sea-Bird temperature and conductivity sensors were mounted along an electro-mechanical mooring cable, and two WHOI Bio-Optical systems were placed in the mooring line at 10- and 40-m depth (Figure 11, Table 10). The $\mathrm{T} / \mathrm{C}$ sensor pairs were mounted perpendicular to the mooring cable so they were horizontal for maximum flushing of the conductivity and temperature sensors. The sensors were powered down the cable (under control of the data system) and powered up every minute and sampled. The meteorology sensors were sampled at a basic interval of 10 seconds. All sensors were averaged in the buoy to one hour values recorded internally and telemetered to shore via the GOES satellite.

Table 9: NMFS VACM with Added Conductivity and Light Specifications

\begin{tabular}{|c|c|c|c|}
\hline Parameter & Sensor Type & Accuracy & Sampling Method \\
\hline Speed & Savonius Rotor & $\pm 1 \mathrm{~m} / \mathrm{s}$ & Vector Average \\
\hline Direction & $\begin{array}{c}\text { Vane with Vane } \\
\text { Follower, Compass } \\
\text { WHOI/EG\&G }\end{array}$ & $\pm 3^{\circ}$ & Vector Average \\
\hline Temperature & $\begin{array}{c}\text { Thermistor } \\
\text { Thermometrics } \\
4 \mathrm{~K} \Omega @ 25^{\circ} \mathrm{C} \\
\end{array}$ & $\pm 0.005^{\circ} \mathrm{C}$ & $\begin{array}{l}\text { Averaged Over a } \\
\text { Half-Record Time }\end{array}$ \\
\hline Conductivity & Sea-Bird SBE-4 & $\pm .001 \mathrm{~S} / \mathrm{m} / \mathrm{mo}$ & $\begin{array}{l}\text { Averaged Over } \\
\text { Record Rate }\end{array}$ \\
\hline Transmission & Sea Tech $25-\mathrm{cm}$ & $\pm 3 \%$ & $\begin{array}{l}\text { Averaged Over } 7.5 \text { - } \\
\text { Minute Samples }\end{array}$ \\
\hline
\end{tabular}

Table 10: SF and CR Sensor Specifications

\begin{tabular}{|l|l|c|c|}
\hline \multicolumn{1}{|c|}{ Parameter } & \multicolumn{1}{|c|}{ Sensor Type } & Accuracy & Record Time \\
\hline Air Temperature & $\begin{array}{l}\text { Thermometrics } \\
\text { Thermistor/Gill } \\
\text { Radiation Shield }\end{array}$ & $\begin{array}{c} \pm 0.2^{\circ} \mathrm{C} \\
10 \mathrm{~K} \Omega @ 25^{\circ} \mathrm{C}\end{array}$ & $\begin{array}{l}\text { Hourly Averages of } \\
1-\text { Minute Samples }\end{array}$ \\
\hline PAR & $\begin{array}{l}\text { LiCor UWQ - Cosine } \\
\text { Underwater Quantum } \\
\text { Sensor }\end{array}$ & $\pm 10 \mu \mathrm{moles} / \mathrm{s} / \mathrm{m}^{2}$ & $\begin{array}{l}\text { Hourly Averages of } \\
10-\text { Second Samples }\end{array}$ \\
\hline Temperature & Sea-Bird SBE-3 & $\pm 0.002^{\circ} \mathrm{C} / \mathrm{yr}$ & $\begin{array}{l}\text { Hourly Averages of } \\
1-\text { Minute Samples }\end{array}$ \\
\hline Conductivity & Sea-Bird SBE-4 & $\pm 0.001 \mathrm{~S} / \mathrm{m} / \mathrm{mo}$ & $\begin{array}{l}\text { Hourly Averages of } \\
1-\text { Minute Samples }\end{array}$ \\
\hline
\end{tabular}


The WHOI Bio-Optical units were developed at WHOI by P. Fucile, J. Dean and J. Irish to measure and record temperature, conductivity, scalar PAR (4 pi steradians), chlorophyll-a fluorescence, and light transmission (Figure 12, Table 11). The instrumentation was mounted in an in-line frame that was separate from the buoy data system and power. It had an internal power and recording system that powered all sensors and sampled every 3.75 minutes, and stored the data internally on PCMCIA flash media. The limitation to these observations was the bio-fouling of the optical windows, which generally limited useful records to about 120 days of the 150- to 180-day deployments. The recording system functioned well, but the averaging circuit that averaged the PAR sensor over the sample interval had a serious overflow problem that required much effort to unscramble the record, and still left uncorrected overflow errors in the record.

The science mooring utilized a elastic tether to supply compliance to the mooring and provide a better riding platform and reduce shock loading in the mooring. This worked well (Irish, 2000), but prevented the lower part of the water column from being sampled on this mooring. To provide deeper measurements, a bottom package containing a new bottom-mounted RD Instruments (RDI) $300-\mathrm{kHz}$ BroadBand Acoustic Doppler Current Profiler (BB ADCP) and Sea-Bird temperature and Paroscientific pressure sensors was deployed close to the central science mooring (between the science mooring and a guard mooring. The ADCP and bottom pressure instrumentation were set to sample an average over the hourly record intervals (Table 12). The new RDI BB ADCP failed during both deployments in the first year due to hardware problems in the instrumentation. Records of only a few hours to several days were obtained in each case. The bottom pressure/temperature instrumentation also failed early, providing data for only a few days in the first deployment and 35 days in the second deployment.

Table 11: WHOI Bio-Optical Package Specifications

\begin{tabular}{|l|l|c|c|}
\hline \multicolumn{1}{|c|}{ Parameter } & \multicolumn{1}{c|}{ Sensor Type } & Accuracy & Record Time \\
\hline Temperature & Sea-Bird SBE-3 & $\pm 0.002^{\circ} \mathrm{C} / \mathrm{yr}$ & 3.75 -Minute Samples \\
\hline Conductivity & Sea-Bird SBE-4 & $\pm 0.001 \mathrm{~S} / \mathrm{m} / \mathrm{mo}$ & 3.75 -Minute Samples \\
\hline PAR & $\begin{array}{l}\text { LiCor SPQ - Scalar } \\
\text { Spherical Underwater } \\
\text { Quantum Sensor }\end{array}$ & $\pm 10 \mu$ moles $/ \mathrm{s} / \mathrm{m}^{2}$ & 3.75 -Minute Samples \\
\hline Chlorophyll-a & $\begin{array}{l}\text { Sea Tech } \\
\text { Fluorometer }\end{array}$ & $\begin{array}{c}\text { Not } \\
\text { Calibrated }\end{array}$ & 3.75 -Minute Samples \\
\hline Light Transmission & $\begin{array}{l}\text { Sea Tech } \\
\text { 25-cm Beam } \\
\text { Transmissometer }\end{array}$ & $\pm 3 \%$ & 3.75 -Minute Samples \\
\hline
\end{tabular}




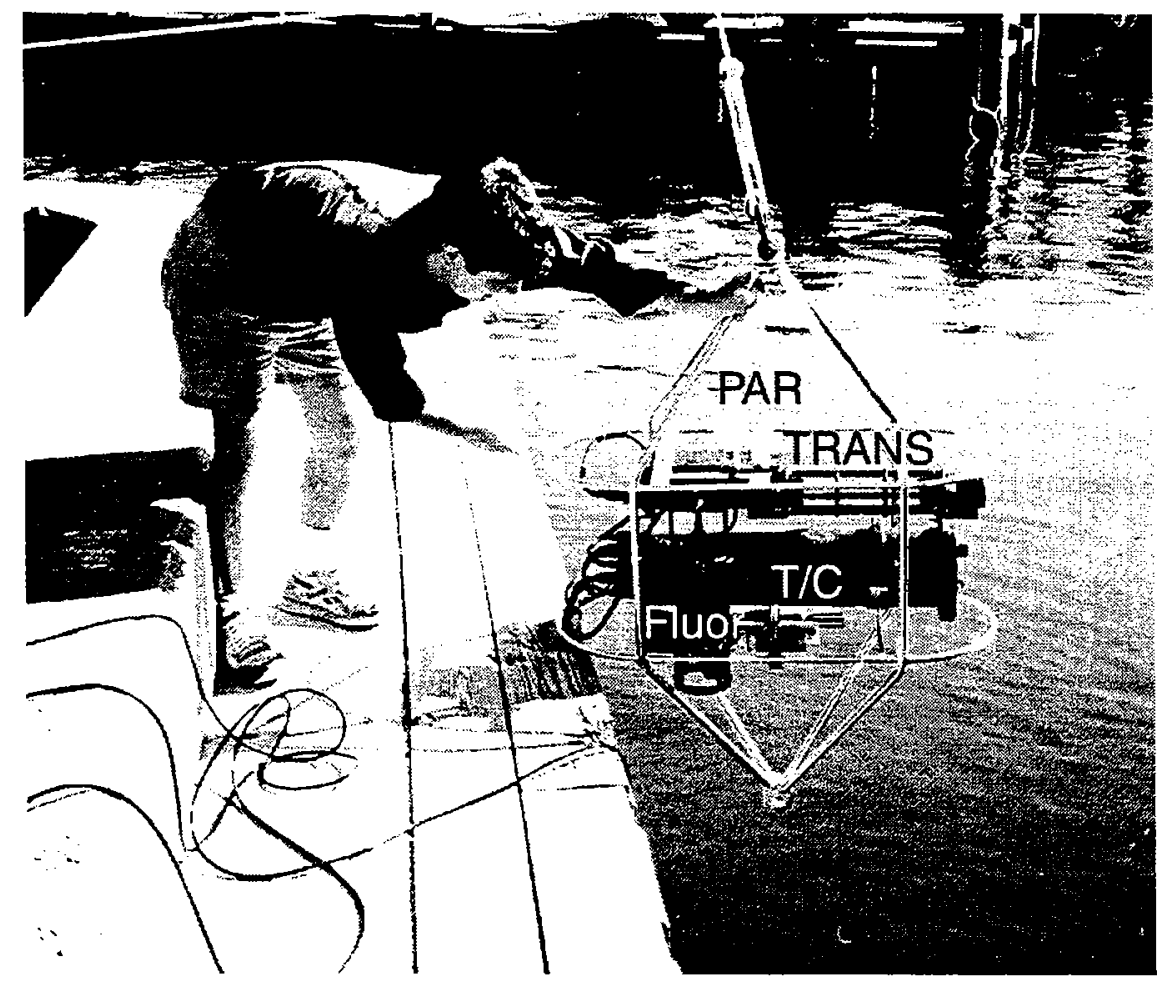

Figure 12: Photograph of WHOI Bio-Optical Unit

Table 12: Bottom-Mounted ADCP/P/T/C Specifications

\begin{tabular}{|l|l|c|c|}
\hline Parameter & Sensor Type & Accuracy & Record Time \\
\hline Temperature & Sea-Bird SBE-3 & $\pm 0.002^{\circ} \mathrm{C} / \mathrm{yr}$ & $\begin{array}{c}\text { Hourly Averages } \\
\text { of Frequency }\end{array}$ \\
\hline Pressure & $\begin{array}{l}\text { Paroscientific } \\
\text { Quartz Crystal }\end{array}$ & $\pm 0.01 \mathrm{db}$ & $\begin{array}{c}\text { Hourly Averages } \\
\text { of Frequency }\end{array}$ \\
\hline Velocity & $\begin{array}{l}\text { RD Instruments } \\
\text { Broadband ADCP } \\
300 \mathrm{KHz}\end{array}$ & $\pm 0.5 \mathrm{~cm} / \mathrm{s}$ & $\begin{array}{c}\text { Hourly Averages } \\
\text { of } 2-\mathrm{sec} \text { Pings }\end{array}$ \\
\hline
\end{tabular}

The elastic tether on the central science surface mooring was cut in early March by fishing activity. All the instrumentation was recovered intact and the SF science mooring was redeployed in April. To provide some information on the temperature structure while the science mooring was being repaired, one of the guard moorings was instrumented with four PMEL temperature recorders (Table 13). These instruments worked well, and indicated that in mid-September the surface guard buoy was pulled under to where the Surlyn foam flotation was compressed to the point that the 
buoy lost buoyancy and sank. The mooring was recovered while dragging for other instrumentation in the area and the temperature sensors recovered. The Surlyn foam slowly returned to nearly its uncompressed condition over the next few months.

Table 13: PMEL Temperature Recorder Specifications

\begin{tabular}{|l|l|c|c|}
\hline Parameter & Sensor Type & Accuracy & Record Time \\
\hline Temperature & Thermistor & $\pm .02^{\circ} \mathrm{C}$ & One Sample at Record Time \\
\hline
\end{tabular}

Table 14a: Aanderaa RCM Specifications

\begin{tabular}{|l|l|c|c|}
\hline \multicolumn{1}{|c|}{ Parameter } & \multicolumn{1}{|c|}{ Sensor Type } & Accuracy & $\begin{array}{c}\text { Record } \\
\text { Time }\end{array}$ \\
\hline Current Speed & Paddle Wheel Rotor & $\pm 1.3 \%$ & $60 \mathrm{Min}$ \\
\hline Current Direction & Vane/Magnetic Compass & $\pm 5 \%$ & $60 \mathrm{Min}$ \\
\hline Temperature & Thermistor & $\pm 0.04^{\circ} \mathrm{C}$ & $60 \mathrm{Min}$ \\
\hline Conductivity & Inductive Cell & $\pm 0.07 \mathrm{mmho} \mathrm{cm}^{-1}$ & $60 \mathrm{Min}$ \\
\hline
\end{tabular}

Table 14b: Aanderaa Tide Gauge Specifications

\begin{tabular}{|l|l|l|l|}
\hline Parameter & Sensor Type & Accuracy & $\begin{array}{c}\text { Record } \\
\text { Time }\end{array}$ \\
\hline Pressure & $\begin{array}{l}\text { Paroscientific } \\
\text { Quartz Crystal }\end{array}$ & $\begin{array}{c} \pm 0.01 \% \mathrm{FS} \\
\text { or } \pm 2.7 \mathrm{db}\end{array}$ & $60 \mathrm{Min}$ \\
\hline Temperature & Thermistor & $\pm 0.04^{\circ} \mathrm{C}$ & $60 \mathrm{Min}$ \\
\hline
\end{tabular}

\section{$2.5 \mathrm{CR}$}

The WHOI long-term CR surface mooring was first deployed as an engineering test measuring mooring tension (with a load cell in the mooring line) to verify modeling of compliant elastic mooring systems in shallow water environments with strong currents and high waves. The engineering test was successful, the mooring remaining in position during two deployments for 11 months. The engineering data (sampled at $1 \mathrm{~Hz}$ and used to compute one hour average mean and standard deviation tensions) showed low-peak tensions (less than $1400 \mathrm{lbs}$ ), mainly due to tidal currents and lower high-frequency wave-induced tensions (Irish and Kery, 1996; Irish, 1997; Paul, et al., 1999). 
During the winter deployment, the CR buoy supported surface sensors for incoming PAR, air temperature, and surface water temperature and salinity. The system worked well until January when the load cell failed, shorted out the system power, and ended data collection. During the spring/summer deployment, the load cell was omitted and a WHOI Bio-Optical package was added at 10-m depth. All sensors returned good data during the this deployment.

The water depth at $\mathrm{CR}$ was $43 \mathrm{~m}$, but the surrounding topography varied greatly due to large amplitude ( $\sim 8-15-\mathrm{m}$ high) sand/gravel waves and ridges. The mooring was set in a trough, but no apparent relative movement of the nearby ridges was observed during the deployment. These large-scale roughness elements kept the water well mixed, and little stratification was observed over the summer between the surface and 10-m temperature sensors.

\subsection{NP}

BIO deployed two instrumented subsurface moorings at the Northeast Peak site NP (Figure 14). One supported a near-surface Aanderaa Recording Current Meter (RCM) at $23 \mathrm{~m}$ while the other deeper mooring supported RCMs at 37 and $59 \mathrm{~m}$, plus an Aanderaa tide gauge (TG) mounted on the anchor to measure bottom pressure. The water depth at NP was $73 \mathrm{~m}$. The RCMs were set to record hourly values of current, temperature and conductivity, while the TG recorded pressure and temperature. The accuracies of these measurements, derived from laboratory calibrations and manufacturer's specifications, are given in Table 14. The two subsurface moorings were surrounded by a triangular array of three surface guard buoys. All three RCMs returned complete records, but the TG battery was dead on recovery. Also there was evidence of biological fouling in all of the conductivity cells.

\subsection{NDBC Environmental Buoys}

Data from NDBC environmental buoys located on eastern Georges Bank, Nantucket Shoals, and the central Gulf of Maine were included in the GBSS moored array data set. Each buoy has sensors for wind speed and direction and air and water temperatures, and transmits data hourly to NOAA where the data are quality-controlled and archived (Table 15). In addition, the Georges Bank buoy measured surface water height variability with an accelerometer and recorded significant wave height and mean and significant wave periods. Due to sensor and transmission failures, large data gaps occurred in these time series. Table 16 gives the sensor heights for the different buoys and the periods when good data were returned are shown in Figure 3. 


\section{WHOI Long-term Mooring at CR}

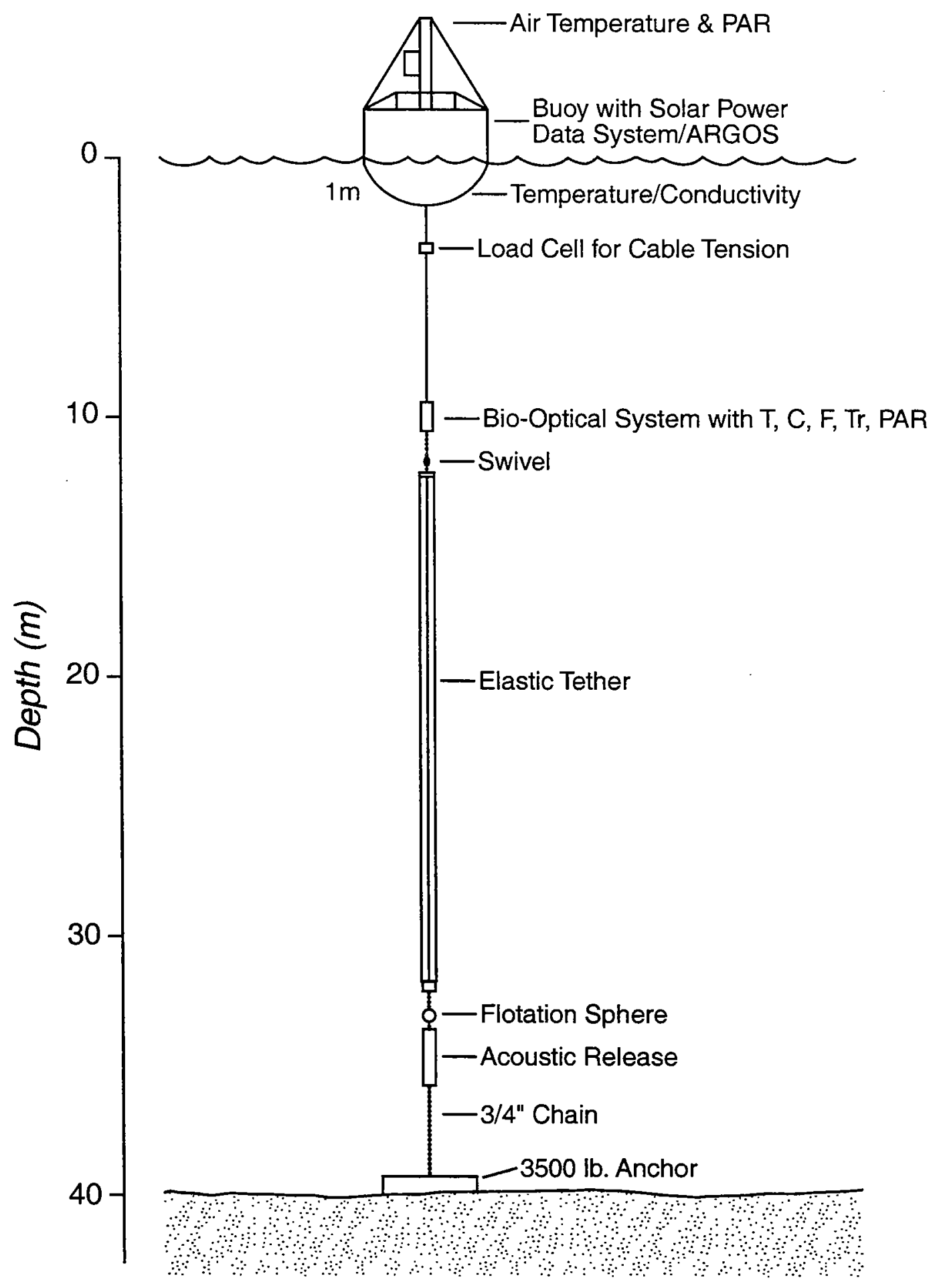

Figure 13: CR Mooring Diagram 


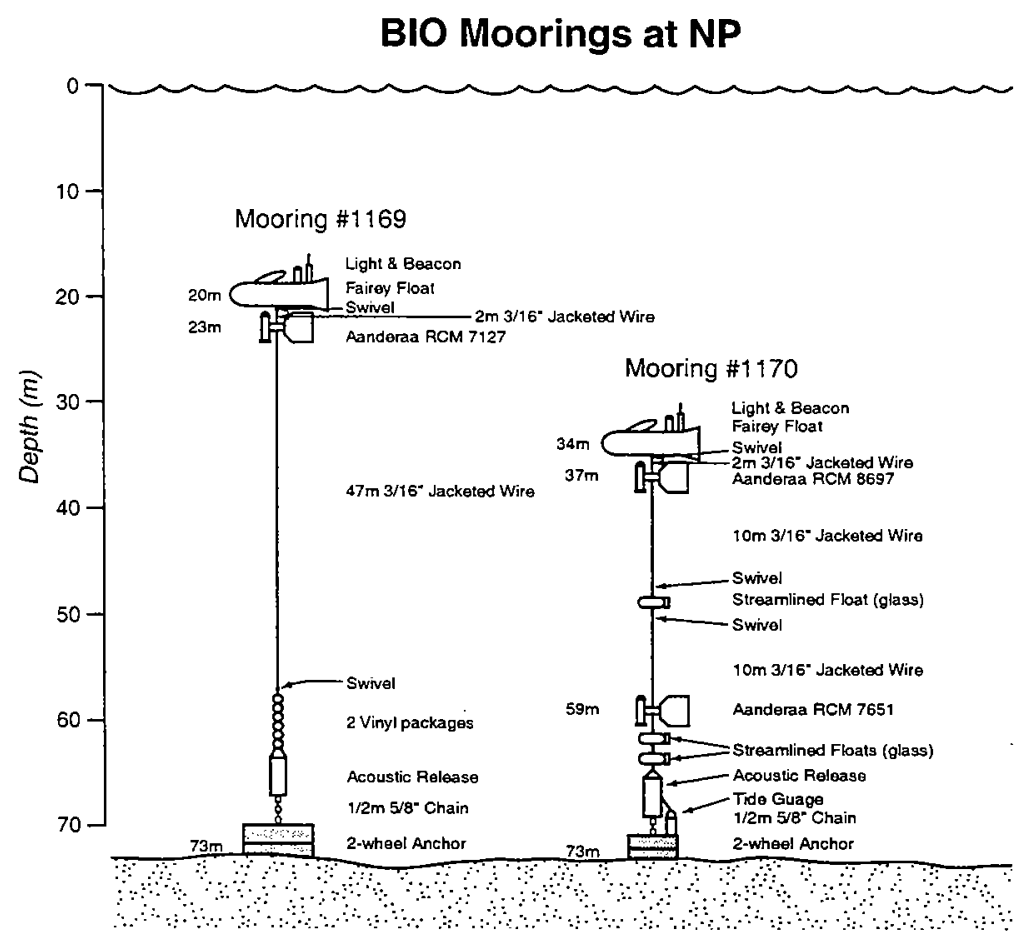

Figure 14: NP Mooring Diagram

Table 15: NDBC Environmental Buoy Specifications

\begin{tabular}{|l|l|c|c|}
\hline \multicolumn{1}{|c|}{ Parameter } & \multicolumn{1}{|c|}{ Sensor-Type } & Accuracy & Record Time \\
\hline Wind Speed & Vane-Directed Impeller & $\pm 1 \mathrm{~m} / \mathrm{s}$ & $\begin{array}{c}8 \text { Min Vector Aver- } \\
\text { age Prior to Hour }\end{array}$ \\
\hline Wind Direction & Vane/Magnetic Compass & $\pm 10^{\circ}$ & $\begin{array}{c}8 \text { Min Vector Aver- } \\
\text { age Prior to Hour }\end{array}$ \\
\hline Air Temperature & Thermistor & $\pm 1^{\circ} \mathrm{C}$ & $\begin{array}{c}8 \text { Min Vector Aver- } \\
\text { age Prior to Hour }\end{array}$ \\
\hline Barometric Pressure & Variable Capacitance & $\pm 1 \mathrm{mbar}$ & $\begin{array}{c}8 \text { Min Vector Aver- } \\
\text { age Prior to Hour }\end{array}$ \\
\hline Water Temperature & Thermistor & $\pm 1^{\circ} \mathrm{C}$ & $\begin{array}{c}8 \text { Min Vector Aver- } \\
\text { age Prior to Hour }\end{array}$ \\
\hline Significant Wave Height* & Accelerometer & $\pm 0.2 \mathrm{~m}$ & $\begin{array}{c}20 \text { Min Sample } \\
\text { Prior to Hour }\end{array}$ \\
\hline Dominant Wave Period & Accelerometer & $\pm 1-\mathrm{sec}$ & $\begin{array}{c}20 \text { Min Sample } \\
\text { Prior to Hour }\end{array}$ \\
\hline
\end{tabular}

*Significant wave height is calculated as the highest one-third of all the wave heights during the 20-min sampling period. Dominant wave period is the period with the maximum energy during the sampling period. 
Table 16: NDBC Buoy Sensor Heights

\begin{tabular}{|ll|l|c|c|c|c|c|}
\hline \multicolumn{1}{|c|}{ Station } & Hull Type & Wind $(\mathbf{m})$ & BP $_{(\mathbf{m})}$ & AT $_{(\mathbf{m})}$ & WT $_{(\mathbf{m})}$ & Wave \\
\hline 44005 & Gulf of Maine & NOMAD & 5.0 & SL & 4.0 & -1.0 & In Hull \\
44008 & Nantucket Shoals & USCG LNG & 13.8 & SL & 11.4 & -1.1 & In Hull \\
44011 & Georges Bank & NOMAD & 5.0 & SL & 4.0 & -1.0 & In Hull \\
\hline
\end{tabular}

${ }^{1}$ NOMAD: (Navy Oceanographic and Meteorological Automatic Device) is a boat-shaped hull 6-m long and 3-m wide.

${ }^{2}$ LNG: (Large Navigational Buoy) is a $12-\mathrm{m}$-diameter discus hull.

\section{Summary of GBSS Moored Instrumentation}

A summary of the instrumentation deployed in the combined GBSS array is given in Table 17, with the exception of the two BASS tripods deployed at ST1, which is described in Section 2.2. The instrument type and ID number, variable measured, sensor height, depth below sea level, record rate, and comments about performance are included. Not included in this table are the SF Bottom ADCP instrument package that returned no data and the ST1 BASS tripods that are described in detail in Section 2.2 .

Table 17: Summary of Moored Instrumentation Deployed in the U.S. GLOBEC Georges Bank Stratification Study at the ST1, ST2, CR, SF, and NP Sites

\begin{tabular}{|c|c|c|c|c|}
\hline $\begin{array}{c}\text { Instrument } \\
\text { Type-\# }\end{array}$ & $\begin{array}{l}\text { Variable } \\
\text { Measured }\end{array}$ & $\begin{array}{c}\text { Sensor } \\
\text { Height }(\mathrm{cm}) \\
\text { Depth }(\mathrm{m})\end{array}$ & $\begin{array}{l}\text { Record } \\
\text { Rate } \\
\text { (min) }\end{array}$ & Comments \\
\hline \multicolumn{5}{|c|}{ ST1 Surface Mooring: WHOI Mooring \# 973} \\
\hline \multirow[t]{2}{*}{ VAWR-707WR } & WS & $297.4 \mathrm{~cm}$ & 15.0 & vaWR Cup Assembly \\
\hline & WD & $270.7 \mathrm{~cm}$ & 15.0 & Replaced June 11, 1995 \\
\hline $\mathrm{T}-5861$ & AT & $230.5 \mathrm{~cm}$ & 15.0 & \\
\hline S-28298 & Sw & $304.4 \mathrm{~cm}$ & 15.0 & \\
\hline $\mathrm{L}-28379$ & $\mathrm{LW}$ & $305.5 \mathrm{~cm}$ & 15.0 & \\
\hline$v-024$ & $\mathrm{RH}$ & $231.7 \mathrm{~cm}$ & 15.0 & \\
\hline 48395 & AP & $238.3 \mathrm{~cm}$ & 15.0 & \\
\hline $\mathrm{T}-5575$ & WT & $1.0 \mathrm{~m}$ & 15.0 & \\
\hline IMET Logger-290 & ws & $287.2 \mathrm{~cm}$ & 1.0 & \\
\hline WND113/108 & WD & - & 1.0 & \\
\hline TMP104 & $\mathrm{AT}$ & $237.6 \mathrm{~cm}$ & 1.0 & \\
\hline swR002 & SW & $305.0 \mathrm{~cm}$ & 1.0 & \\
\hline LWR006 & LW & $308.5 \mathrm{~cm}$ & 1.0 & LWR006/003/006 Note 3 \\
\hline HRH110 & RH & $237.7 \mathrm{~cm}$ & 1.0 & \\
\hline PRC109 & $\mathrm{RF}$ & $273.9 \mathrm{~cm}$ & 1.0 & \\
\hline BPR 110 & $\mathrm{AP}$ & $244.2 \mathrm{~cm}$ & 1.0 & \\
\hline $\mathrm{T}-109$ & WT & $-1.0 \mathrm{~m}$ & 1.0 & \\
\hline SeaCat-1803 & $\mathrm{WT}, \mathrm{CD}$ & -1.5 & 1.5 & \\
\hline TPOD-3506 & WT & -2.0 & 30.0 & ............ (Continued) \\
\hline
\end{tabular}


Table 17: Summary of Moored Instrumentation (Continued)

\begin{tabular}{|c|c|c|c|c|}
\hline $\begin{array}{c}\text { Instrument } \\
\text { Type-\# }\end{array}$ & $\begin{array}{l}\text { Variable } \\
\text { Measured }\end{array}$ & $\begin{array}{c}\text { Sensor } \\
\text { Height }(\mathrm{cm}) \\
\text { Depth }(\mathrm{m})\end{array}$ & $\begin{array}{c}\text { Record } \\
\text { Rate } \\
\text { (min) }\end{array}$ & Comments \\
\hline \multicolumn{5}{|c|}{ ST1 Surface Mooring: WHOI Mooring \# 973: (Continued) } \\
\hline MTR-3246 & WT & -3.5 & 30.0 & \\
\hline \multirow[t]{2}{*}{ VMCM-020 } & VEL & -5.2 & 7.5 & \\
\hline & WT & -5.7 & 7.5 & \\
\hline SeaCat-1736 & $\mathrm{WT}, \mathrm{CD}$ & -6.2 & 1.5 & \\
\hline \multirow[t]{2}{*}{ VMCM-040 } & VEL & -7.5 & 7.5 & \\
\hline & WT & -8.0 & 7.5 & \\
\hline \multirow[t]{2}{*}{ VMCM-044 } & VEL & -10.0 & 7.5 & \\
\hline & WT & -10.5 & 7.5 & \\
\hline SeaCat-1819 & $\mathrm{CD}, \mathrm{WT}$ & -11.1 & 1.5 & \\
\hline TPOD-4482 & $\mathrm{WT}$ & -12.8 & 30.0 & \\
\hline \multirow[t]{2}{*}{ VMCM-027 } & VEL & -14.0 & 7.5 & \\
\hline & WT & -14.50 & 7.5 & \\
\hline TPOD-3258 & WT & -17.0 & 30.0 & Data (Memory) Lost \\
\hline \multirow{2}{*}{ VMCM-055 } & VEL & -19.0 & 7.5 & \\
\hline & WT & -19.5 & 7.5 & \\
\hline TPOD-3831 & WT & -22.5 & 30.0 & \\
\hline \multirow[t]{2}{*}{ VMCM-026 } & VEL & -25.0 & 7.5 & \\
\hline & WT & -25.50 & 7.5 & \\
\hline SeaCat-1735 & $\mathrm{WT}, \mathrm{CD}$ & -26.1 & 1.5 & \\
\hline TPOD-4494 & WT & -28.8 & 30.0 & \\
\hline \multirow[t]{2}{*}{ VMCM-031 } & VEL & -31.0 & 7.5 & \\
\hline & WT & -31.5 & 7.5 & \\
\hline TPOD-3701 & WT & -34.5 & 30.0 & Data (Memory) Lost \\
\hline \multirow[t]{2}{*}{$\mathrm{VMCM}-045$} & VEL & -37.0 & 7.5 & \\
\hline & WT & -37.5 & 7.5 & \\
\hline TPOD-3833 & WT & -40.8 & 30.0 & \\
\hline \multicolumn{5}{|c|}{ ST1 Subsurface Mooring: WHOI Mooring \# 974} \\
\hline \multirow[t]{2}{*}{ VMCM-053 } & VEL & -45.7 & 7.5 & \\
\hline & WT & -46.2 & 7.5 & \\
\hline SeaCat-1818 & $\mathrm{ST}, \mathrm{CD}$ & 46.8 & 1.5 & Short \\
\hline \multirow[t]{2}{*}{ VMCM-054 } & VEL & -52.2 & 7.5 & No Data, Tape Stopped \\
\hline & WT & -52.7 & 7.5 & No Data \\
\hline \multirow[t]{2}{*}{ VMCM-054 } & VEL & -58.2 & 7.5 & No Data \\
\hline & WT & -58.7 & 7.5 & No Data \\
\hline TPOD-4485 & WT & -61.7 & 30.0 & \\
\hline \multirow[t]{2}{*}{ VMCM-043 } & VEL & -64.2 & 7.5 & \\
\hline & WT & -64.7 & 7.5 & \\
\hline SeaCat-1820 & $\mathrm{WT}, \mathrm{CD}$ & -65.3 & 1.5 & Short \\
\hline TPOD-3830 & WT & -67.7 & 30.0 & \\
\hline \multirow[t]{2}{*}{ VMCM-008 } & VEL & -70.2 & 7.5 & \\
\hline & wT & -70.7 & 7.5 & No Temperature Data \\
\hline
\end{tabular}


Table 17: Summary of Moored Instrumentation (Continued)

\begin{tabular}{|c|c|c|c|c|}
\hline $\begin{array}{l}\text { Instrument } \\
\text { Type-\# }\end{array}$ & $\begin{array}{l}\text { Variable } \\
\text { Measured }\end{array}$ & $\begin{array}{c}\text { Sensor } \\
\text { Height }(\mathrm{cm}) \\
\text { Depth }(\mathrm{m})\end{array}$ & $\begin{array}{l}\text { Record } \\
\text { Rate } \\
\text { (min) }\end{array}$ & Comments \\
\hline \multicolumn{3}{|c|}{ ST2 Surface Mooring } & & \multirow[t]{7}{*}{ Mooring Cut July 27} \\
\hline SeaCat-1843 & $\mathrm{WT}, \mathrm{CD}$ & -1.0 & 7.5 & \\
\hline TPOD-262 & WT & -5.0 & 30.0 & \\
\hline Minilog & WT & -5.0 & 60.0 & \\
\hline SeaCat-359 & $\mathrm{WT}, \mathrm{CD}$ & -10.0 & 7.5 & \\
\hline \multirow[t]{2}{*}{ VMCM-773 } & VEL & -15.0 & 7.5 & \\
\hline & WT & -15.5 & 7.5 & \\
\hline SeaCat-561 & $\mathrm{WT}, \mathrm{CD}$ & -20.0 & 7.5 & \multirow[t]{8}{*}{ Instrument Lost } \\
\hline TPOD-263 & WT & -25.0 & 30.0 & \\
\hline SeaCat-595 & $\mathrm{WT}, \mathrm{CD}$ & -30.0 & 7.5 & \\
\hline \multirow[t]{2}{*}{ VMCM-1759 } & VEL & -35.0 & 7.5 & \\
\hline & WT & -35.5 & 7.5 & \\
\hline TPOD-264 & WT & -38.4 & & \\
\hline \multirow[t]{2}{*}{ VTCT-518 } & VEL & -53.0 & 7.5 & \\
\hline & WT & -53.5 & 7.5 & \\
\hline \multicolumn{5}{|c|}{ CR Surface Mooring } \\
\hline UWQ & PAR & 2.6 & 60.0 & \\
\hline $\mathrm{T}-$ & $\mathrm{AT}$ & 2.7 & 60.0 & \\
\hline $\mathrm{SB}-\mathrm{T} / \mathrm{C}$ & $\mathrm{WT}, \mathrm{CD}$ & -1.0 & 60.0 & \\
\hline Bio-Optical & PAR & -10.0 & 3.75 & \\
\hline \multicolumn{5}{|c|}{ SF Surface Mooring } \\
\hline$T-$ & AT & 2.7 & 60.0 & \\
\hline UWQ & PAR & 2.6 & 60.0 & \\
\hline SB-T- & WT & -1.0 & 60.0 & \\
\hline $\mathrm{SB}-\mathrm{T} / \mathrm{C}$ & $\mathrm{WT}, \mathrm{CD}$ & -5.0 & 60.0 & \\
\hline Bio-Optical & $\mathrm{WT}, \mathrm{CD}, \mathrm{PAR}, \mathrm{TR}, \mathrm{FL}$ & 10.0 & 3.75 & \\
\hline $\mathrm{SB}-\mathrm{T} / \mathrm{C}$ & $\mathrm{WT}, \mathrm{CD}$ & -15.0 & 60.0 & . \\
\hline $\mathrm{SB}-\mathrm{T} / \mathrm{C}$ & $\mathrm{WT}, \mathrm{CD}$ & -20.0 & 60.0 & \\
\hline $\mathrm{SB}-\mathrm{T} / \mathrm{C}$ & WT,CD & -25.0 & 60.0 & \\
\hline $\mathrm{SB}-\mathrm{T} / \mathrm{C}$ & $\mathrm{WT}, \mathrm{CD}$ & -30.0 & 60.0 & \\
\hline $\mathrm{SB}-\mathrm{T} / \mathrm{C}$ & $\mathrm{WT}, \mathrm{CD}$ & -35.0 & 60.0 & \\
\hline Bio-Optical & $\mathrm{WT}, \mathrm{CD}, \mathrm{PAR}, \mathrm{TR}, \mathrm{FL}$ & -40.0 & 3.75 & \\
\hline $\mathrm{SB}-\mathrm{T} / \mathrm{C}$ & $\mathrm{WT}, \mathrm{CD}$ & -45.0 & 60.0 & \\
\hline $\mathrm{SB}-\mathrm{T} / \mathrm{C}$ & $\mathrm{WT}, \mathrm{CD}$ & -50.0 & 60.0 & \\
\hline \multicolumn{5}{|c|}{ SF Bottom ADCP Mooring } \\
\hline $\mathrm{ADCP}$ & VEL & 76.0 & 60.0 & No Data, Both Deployments \\
\hline $\mathrm{P} / \mathrm{T}$ & $\mathrm{BP}, \mathrm{WT}$ & 76.0 & 60.0 & $\begin{array}{l}\text { No Data First Deployment } \\
\text { Short Second Deployment }\end{array}$ \\
\hline
\end{tabular}


Table 17: Summary of Moored Instrumentation (Continued)

\begin{tabular}{|c|c|c|c|c|}
\hline $\begin{array}{c}\text { Instrument } \\
\text { Type-\# }\end{array}$ & $\begin{array}{l}\text { Variable } \\
\text { Measured }\end{array}$ & $\begin{array}{c}\text { Sensor } \\
\text { Height }(\mathrm{m}) \\
\text { Depth }(\mathrm{m})\end{array}$ & $\begin{array}{c}\text { Record } \\
\text { Rate } \\
\text { (min) }\end{array}$ & Comments \\
\hline \multicolumn{5}{|c|}{ SF Surface Guard Mooring } \\
\hline PMEL & WT & 1.0 & 5.0 & \\
\hline PMEL & WT & 10.0 & 5.0 & \\
\hline PMEL & WT & 20.0 & 5.0 & \\
\hline PMEL & WT & 50.0 & 5.0 & \\
\hline \multicolumn{5}{|c|}{ NP Subsurface Mooring, BIO Mooring \#1169 } \\
\hline $\mathrm{RCM}-7127$ & VEL,WT, CD & 23.0 & 60.0 & \\
\hline \multicolumn{5}{|c|}{ NP Subsurface Mooring, BIO Mooring \#1170 } \\
\hline RCM-8697 & VEL,WT,CD & 37.0 & 60.0 & \\
\hline RCM-7651 & VEL, WT, CD & 59.0 & 60.0 & \\
\hline TG-821 & $\mathrm{BP}, \mathrm{WT}$ & 73.0 & 60.0 & Short, Battery Died \\
\hline
\end{tabular}

\section{Footnotes for Table 17}

IMET: Improved Meteorological Recorder

Minilog: VEMCO Temperature Logger

PAR: Photosynthetically Active Radiation

PMEL: Pacific Marine Environmental Laboratory Temperature Recorder

RCM: Aanderaa Recording Current Meter

SB-T/C: Sea-Bird Temperature (SBE-3)/ Conductivity (SBE-4)

SeaCat: Sea-Bird Self-Recording Temperature/Conductivity/Unit

SPQ: LiCor Scalar Spherical Underwater Quantum Sensor

UWQ: LiCor Cosine Underwater Quantum Sensor

VACM: Vector Averaging Current Meter

VAWR: Vector Averaging Wind Recorder Standard Met Package

VEMCO: Minilog Temperature and Depth Logger

VMCM: Standard Vector Measuring Current Meter - Velocity (VEL) and WT

VTCT: VACM with Temperature, Sea-Bird Conductivity Cell, and Transmissometer
AP: Atmospheric Pressure
LW: Long-Wave Radiation
TR: Light Transmission
AT: Air Temperature
RF: Rainfall
WD: Wind Direction
BP: Bottom Water Pressure
RH: Relative Humidity
WS: Wind Speed
CD: Water Conductivity
SW: Short-Wave Radiation
WT: Water Temperature
FL: Fluorescence
TG: Aanderaa Tide Gauge
VEL: Water Velocity 


\section{Data Processing}

This section provides a detailed description of the different data processing methods and evaluation procedures used to produce the final edited basic and hourly meteorological and oceanographic time series for the combined GBSS moored array. The section is organized by mooring sites, since each group was responsible for producing the final data from their moored instrumentation. Greenwich Mean Time (GMT) is used throughout.

\subsection{ST1}

\subsubsection{VAWR and IMET}

The surface 3-m discus buoy at ST1 supported two separate meteorological measurement instruments, the VAWR and IMET, to obtain independent measurements of most variables and improve overall data quality and system reliability. Each system had separate data loggers and a full complement of meteorological sensors, the IMET also recorded precipitation. The VAWR and IMET basic 7.5-minute and 1-minute time series were processed by the WHOI Upper Ocean Processes (UOP) Group, and combined by R. Beardsley and C. Alessi to produce the final time series presented here. The methods used to compare the various VAWR and IMET records and construct the best versions are presented next.

\subsubsection{Wind Speed and Direction}

Both the VAWR 3-cup wind speed sensor and the IMET wind monitor were damaged during the deployment. The VAWR and IMET wind speeds agreed well until April 5 when the IMET speed began to decrease relative to the VAWR. By May 17, the IMET speeds had dropped to zero for extended periods. The VAWR speeds dropped to zero starting May 10. Upon recovery, the IMET propeller had several blades missing and a broken vane, and the VAWR three-cup anemometer was missing two cups. The complete IMET wind monitor and the VAWR wind speed sensor were replaced on July 11 and returned good data to the end of the deployment.

The VAWR wind time series were more complete than the IMET, so the VAWR wind data were combined with wind data from NDBC environmental buoy 44011 to form a complete record for the duration of the VAWR deployment (Figure 15). NDBC 44011, located in $87 \mathrm{~m}$ of water on the southeastern flank of Georges Bank, approximately $87 \mathrm{~km}$ east of the VAWR mooring, was equipped with a R.M. Young wind monitor mounted at a height of $5 \mathrm{~m}$. The NDBC wind record was a vector average of speed and direction samples collected at $1 \mathrm{~Hz}$ between minutes 42 and 50 of each hour. The NDBC 44011 wind series overlapped the VAWR data between February 19 and August 23 (except for one 27-hour gap in the NDBC series starting May 8 which was simply filled with VAWR data). A comparison of the VAWR and NDBC 44011 wind data indicated that the VAWR returned good data for two time periods; an initial "front" 80-day period before the VAWR speed became zero and the "end" 43-day period after the broken VAWR speed sensor was replaced. The "front" and "end" time series were combined into single 122-day VAWR and NDBC 44011 wind series that were used to determine the best regression fit between the two wind speed time series and direction offset. The 15-minute VAWR speed series was subsampled at different time 
offsets to coincide with the hourly NDBC 44011 series and the resultant time series fit to the model

$$
S_{\text {vawr }}=a+b \times S_{n d b c} .
$$

The best regression was found when the NDBC series lagged the VAWR series by 1.25 hours, implying eastward propagation of wind speed events with a mean speed of 38 knots. For this lag, $a=0.77 \pm 0.11 \mathrm{~m} / \mathrm{s}, b=0.83 \pm 0.02$, the correlation coefficient $=0.89$, and the standard deviation between actual and model VAWR speeds is $\pm 1.5 \mathrm{~m} / \mathrm{s}$ (with maximum and minimum differences being $\pm 6.7 \mathrm{~m} / \mathrm{s}$ ) (Figure 16). The decorrelation time scale of the speed difference time series is about 7 hour, with about $88 \%$ of the data normally distributed between $\pm 2 \mathrm{~m} / \mathrm{s}$.

The above regression model was then used to predict or fill the missing VAWR speed data between the "front" and "end" periods. The complex vector correlation computed between the VAWR and NDBC 44011 wind velocities for the combined "front" and "end" period indicated a mean rotation of $5.2^{\circ}$ between the two vector series; this offset was then used to adjust the predicted VAWR wind direction. A composite vector-averaged hourly wind velocity time series was then constructed using the 15-minute VAWR wind data and the wind speed and direction data predicted using the NDBC 44011 data.

The hourly VAWR and IMET vector speeds were compared for the 63-day "front" and 43-day "back" periods when both sensors are known to be working. Linear regression analysis for the model

$$
S_{\text {imet }}=a+b \times S_{v a w r}
$$

gave values for $a$ and $b$ for each period which agreed within the $95 \%$ confidence limits (the values for $b$ differed by less than $0.9 \%$ ), indicating that two completely different IMET and VAWR sensor sets agreed in relative performance to within about $1 \%$. Analysis for the combined series yielded $a=0.17 \pm 0.02 \mathrm{~m} / \mathrm{s}, b=0.893 \pm 0.002$, the correlation coefficient $=0.998$, with a standard deviation between IMET observed and predicted wind speed of $0.19 \mathrm{~m} / \mathrm{s}$. Thus, the IMET vector speed was about $11 \%$ smaller than the VAWR vector speed. The causes of this are not clear.

\subsubsection{Incident Short-Wave Radiation}

The VAWR Eppley model 8-48 and the IMET Eppley PSP pyranometers both returned data for the entire deployment (Figure 17). Both sensors exhibited a $0.6 \%$ decrease in sensitivity over the experiment based on pre- and post- calibrations conducted at Eppley. The hourly incident $S W$ time series agreed well (the correlation coefficient $=0.996$ ) (Figure 18 ), with a record mean difference of only $S W_{v a w r}-S W_{\text {imet }}=-1.8 \pm 0.3 \mathrm{~W} / \mathrm{m}^{2}$ (standard deviation $= \pm 23.9 \mathrm{~W} / \mathrm{m}^{2}$ ). Regression analysis on the hourly and daily average series indicates that over the entire deployment period, the VAWR SW sensor read about $1 \%$ (hourly) to $2 \%$ (daily) higher in comparison with the IMET. Closer inspection of the basic 15-minute data showed that the IMET tended to clip the mid-day short-wave values in comparison to the VAWR and predicted clear sky values (Figure 19). For this reason, the VAWR SW record was used.

\subsubsection{Incident Long-Wave Radiation}

Both IMET and VAWR systems used Eppley model PIR pyranometers to measure incident longwave radiation. The resulting $L W$ time series exhibited good agreement over the first 29-day period until March 1, when the IMET long-wave sensor housing flooded (Figures 20 and 21). During this 

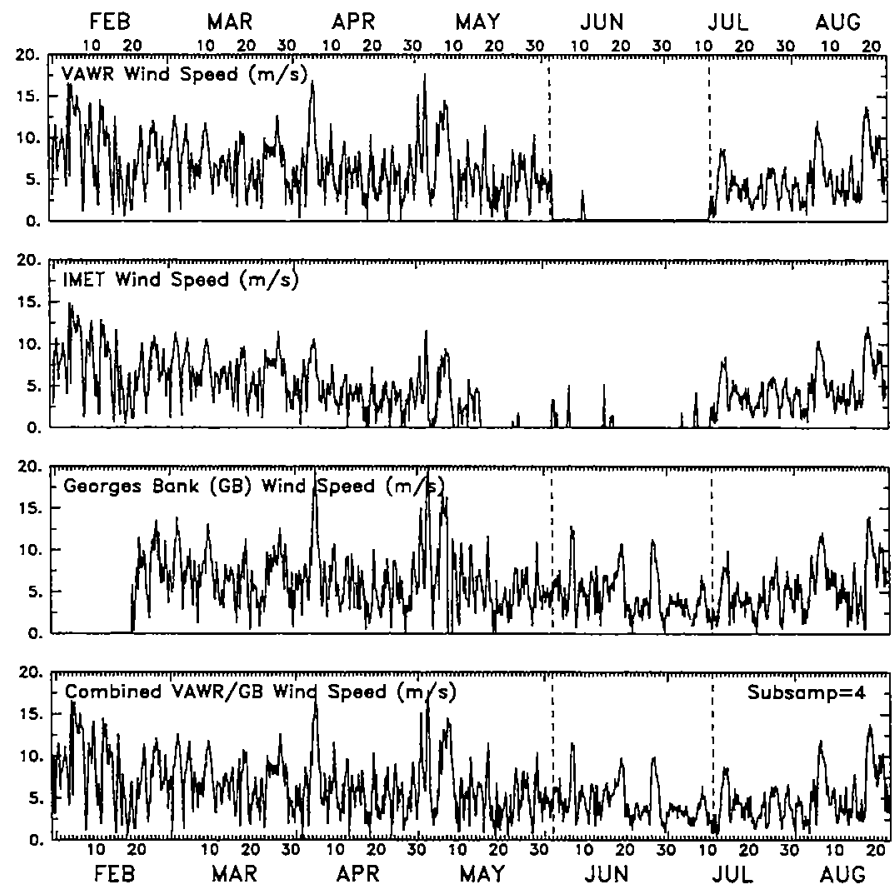

Figure 15: VAWR (top), IMET (second), NDBC 44011 (third), and combined VAWR and NDBC (bottom) vector-averaged wind speed time series.

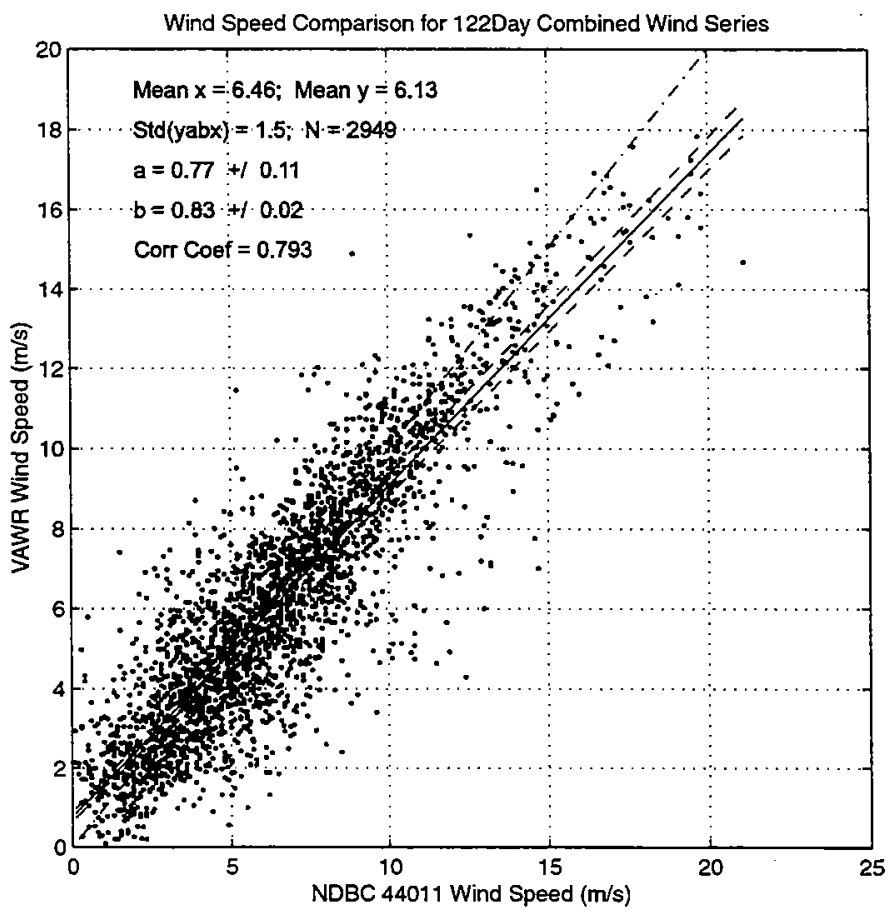

Figure 16: Comparison Between VAWR and NDBC 44011 Vector-Averaged Hourly Wind Speeds 

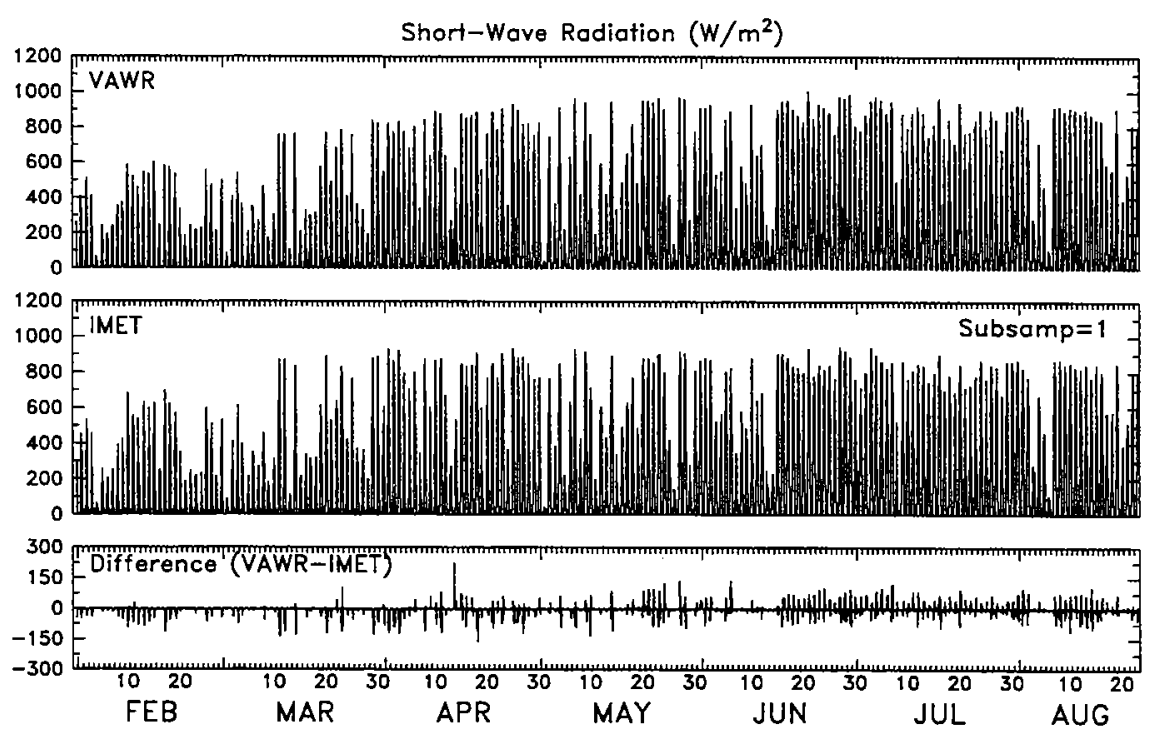

Figure 17: VAWR and IMET Short-Wave Radiation and Difference Time Series

initial period when both sensors returned good data, the two time series differed on average by $3.4 \pm 4.5 \mathrm{~W} / \mathrm{m}^{2}$ during night-time but more during day-time. Regression between the difference time series and insolation indicated that the VAWR long-wave sensor experienced significant internal heating due to daily solar heating (Figure 22). This effect could be significant, corresponding to a difference of $23 \mathrm{~W} / \mathrm{m}^{2}$ when insolation reached $\mathrm{SW}=900 \mathrm{~W} / \mathrm{m}^{2}$. To correct for this effect, a new long-wave time series was constructed using

$$
\begin{aligned}
L W & =L W_{\text {vawr }}-3.0 \text { when } S W<10 \mathrm{~W} / \mathrm{m}^{2}, \\
& =L W_{\text {vawr }}-(1.0+0.0259 \times S W) \text { for } S W>10 \mathrm{~W} / \mathrm{m}^{2} .
\end{aligned}
$$

This new series exhibited significantly less variability at $1 \mathrm{cpd}$.

The IMET long-wave sensor was replaced on April 28 but failed after 4.75 days. During this brief period, the VAWR and IMET data were in good agreement, suggesting that the VAWR and first and second IMET sensors agreed within calibration uncertainty. The broken IMET sensor was replaced again on June 11, and returned data for the rest of the deployment (Figure 20). Unfortunately, the dome thermistor detached from the dome prior to deployment so that the correction for dome temperature was incorrect. This led to the IMET data being consistently higher than the VAWR data, with a mean difference of $L W_{\text {imet }}-L W_{\text {vawr }}=33.1 \pm 7.8 \mathrm{~W} / \mathrm{m}^{2}$ (Figure 21). The VAWR long-wave radiation time series adjusted for short-wave heating was used all subsequent analysis.

\subsubsection{Barometric Pressure}

The IMET and VAWR used identical Paroscientific model 215-AW digiquartz pressure transducers equipped with Gill pressure ports. The two BP time series agreed well, with a mean difference of only $0.07 \mathrm{mb}$, a standard deviation of $0.11 \mathrm{mb}$, and a relative drift of the VAWR from the IMET sensor of $0.14 \mathrm{mb}$ over the 203.9 -day record length. Since neither series looked in error, an average of the two series was used in subsequent analysis. 


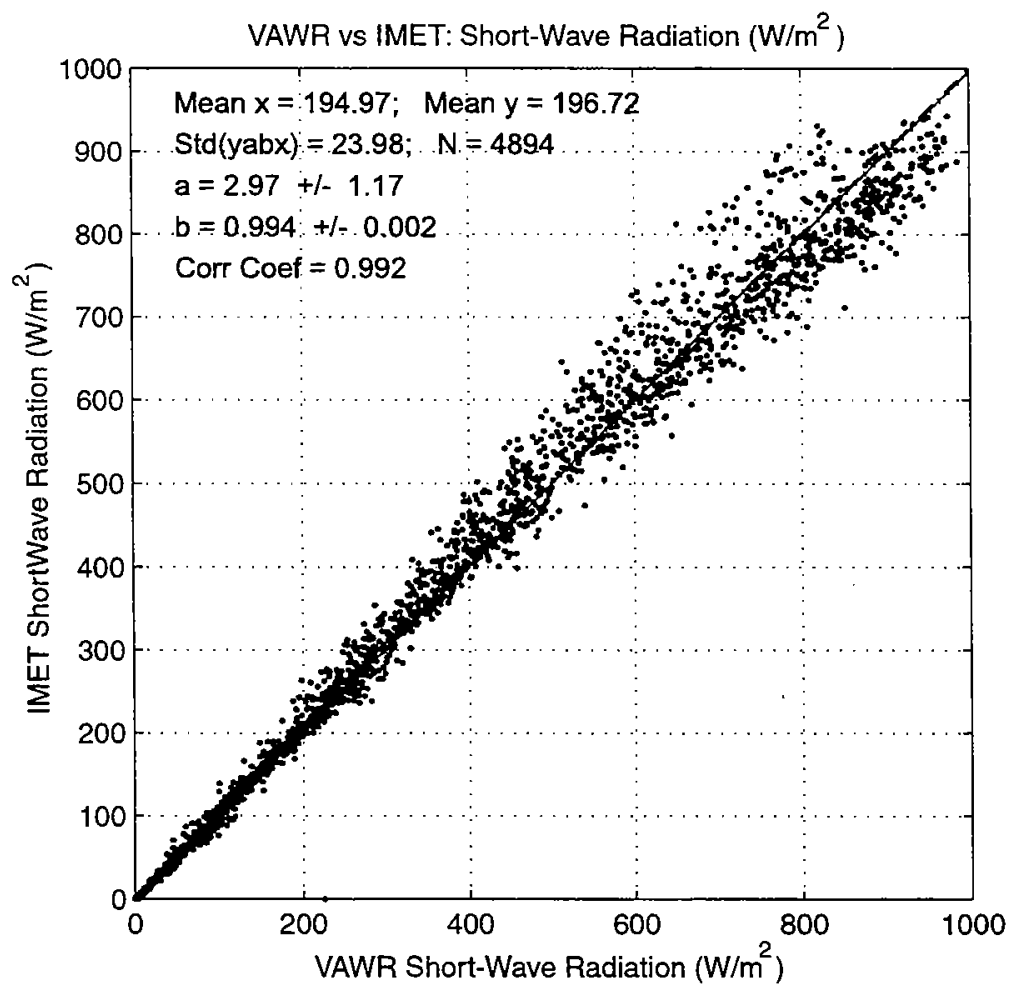

Figure 18: Comparison of VAWR and IMET hourly short-wave radiation time series. Results of regression analysis are shown in upper left.

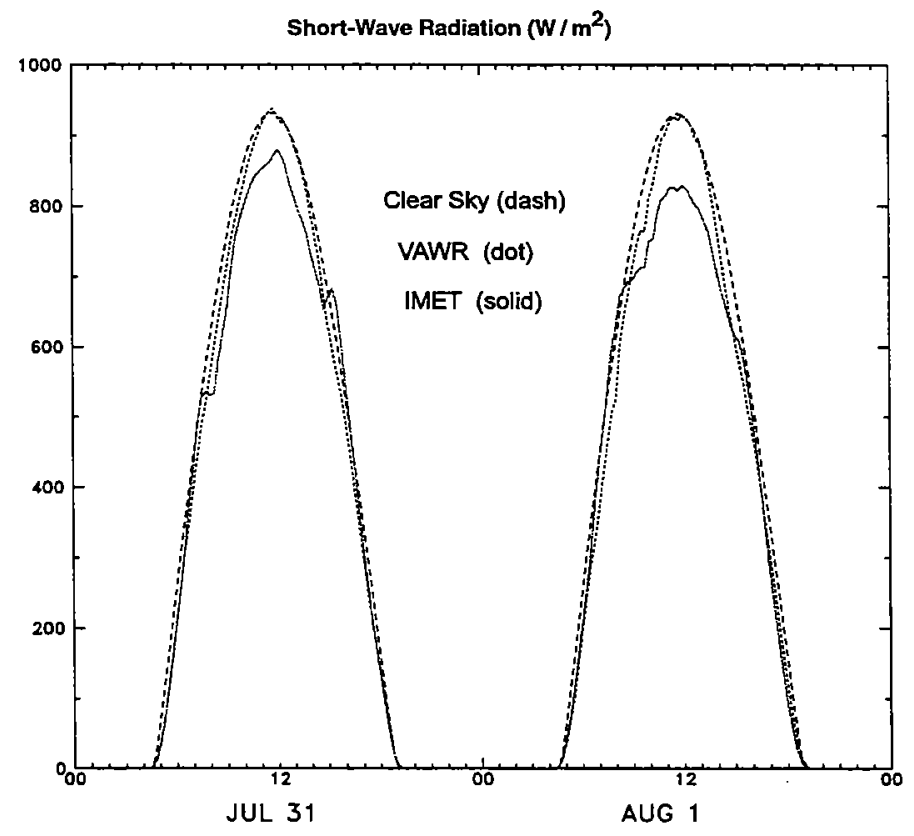

Data are Plotted in Eastern Standard Time

Figure 19: Comparison of VAWR, IMET, and clear sky insolation hourly time series during two days with little or no clouds. 


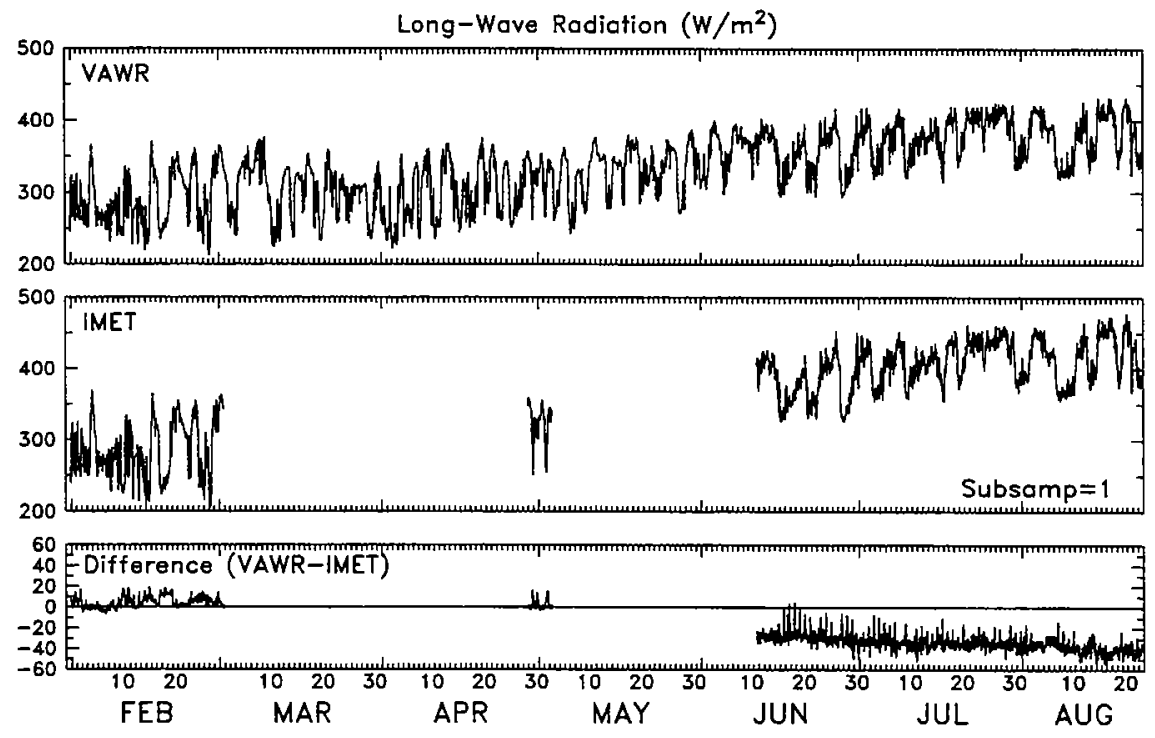

Figure 20: VAWR and IMET Long-Wave Radiation and Difference Time Series

\subsubsection{Relative Humidity}

The VAWR and IMET featured Väisälä Humicap and Rotonics relative humidity sensors, respectively. Both relative humidity records exhibited a tendency for increasing $\mathrm{RH}$ with yearday, with synoptic scale (2-15-day) fluctuations superimposed (Figure 23). While the mean and standard deviation of the difference between the VAWR and IMET RH records were only $2.5 \% \pm 2.9 \%$, the VAWR RH exceeded $100 \%$ about $11.7 \%$ of the record, and also appeared to respond more slowly to variations in $\mathrm{RH}$ than the IMET sensor. A detailed comparison made over the first 87.5 days when both sensors seem to track well (with no values exceeding 100\%) indicated no clear effect of daily solar heating but a weak correlation with wind speed, such that the VAWR RH increased relative to the IMET with increasing wind speed (e.g., the increase was $3.7 \%$ at a wind speed of $10 \mathrm{~m} / \mathrm{s}$ ). The decorrelation time scale for the VAWR minus IMET series was about one day, suggesting that systematic differences between the two $\mathrm{RH}$ records perhaps caused by a systematic instrument error should be lost after about one day. Since the dominant variability in $\mathrm{RH}$ occurs on longer time scales, any systematic problems should be reduced. Since the overall IMET record seemed more responsive, was less sensitive to wind speed, and did not exceed 100\%, the IMET RH time series was chosen for further analysis.

\subsubsection{Air Temperature}

The VAWR used a precision thermistor to measure air temperature while the IMET used a platinum thermometer. The two air temperature records agreed closely, with the VAWR and IMET AT series differing on average by $0.05 \pm 0.16^{\circ} \mathrm{C}$ (Figure 24). While air temperature has a red spectrum with most of its variance in the synoptic 2-10-day band, the difference AT series had a large spectral peak at $1 \mathrm{cpd}$ (due to solar heating of the sensor shield) and most of its variance at frequencies above $1 \mathrm{cpd}$, where the spectrum was flat (i.e., white noise). Comparison of the difference time series versus insolation and wind speed showed the following. During night-time $\left(\mathrm{SW}<10 \mathrm{~W} / \mathrm{m}^{2}\right)$, 


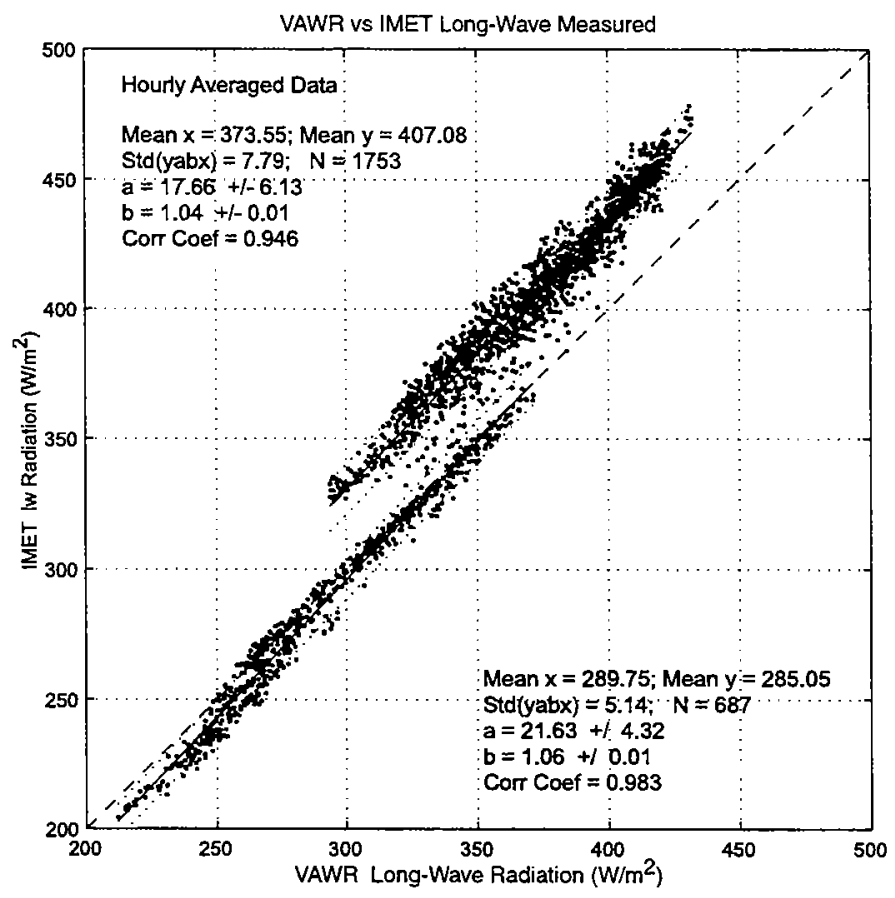

Figure 21: Comparison of VAWR and IMET Long-Wave Radiation During First and Last Measurement Periods

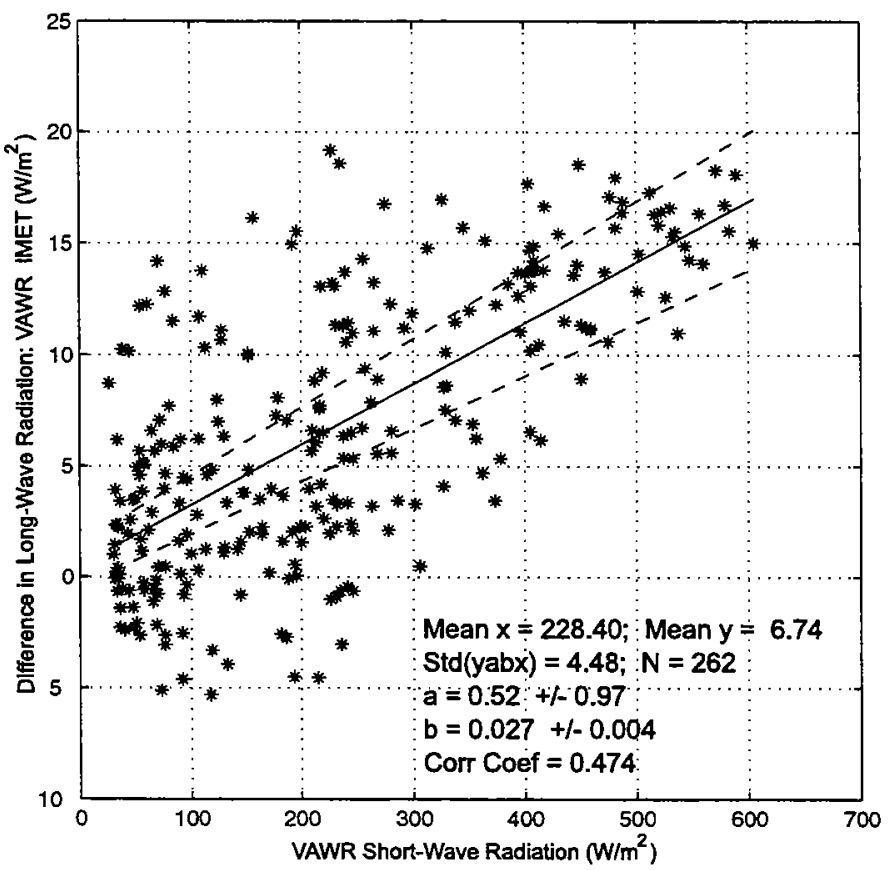

Figure 22: The difference between VAWR and IMET long-wave radiation measurements versus VAWR short-wave radiation during daytime $\left(\mathrm{SW}>25 \mathrm{~W} / \mathrm{m}^{2}\right)$. Only data from initial 28-day period before IMET failure occurred are shown. 


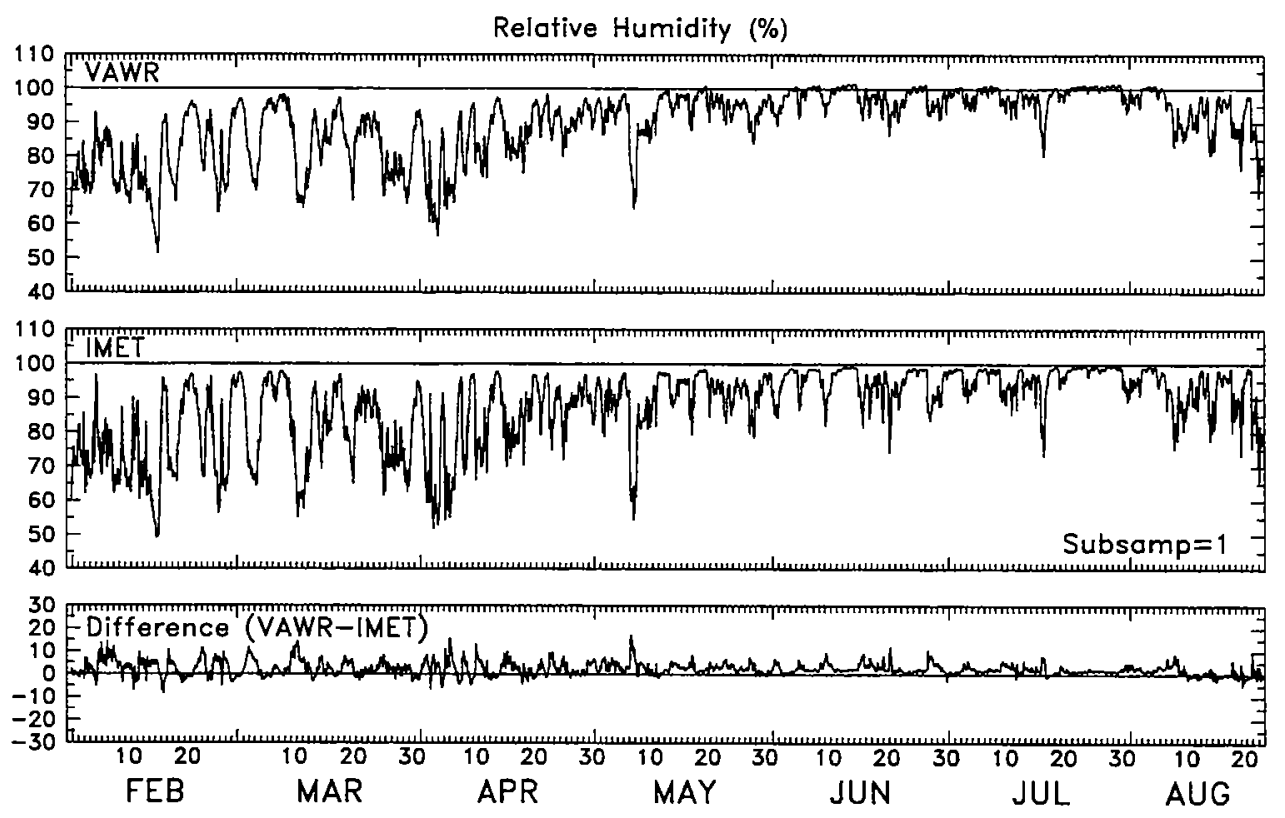

Figure 23: VAWR and IMET Relative Humidity and Difference Time Series

the mean AT difference was $-0.012 \pm 0.083^{\circ} \mathrm{C}$. During the day, the AT difference exhibited a clear dependency on increasing insolation with decreasing wind speed. Regression of the difference versus insolation for $1 \mathrm{~m} / \mathrm{s}$ wind speed bins showed that the largest deviations occurred for wind speeds below $4 \mathrm{~m} / \mathrm{s}$. Below $1 \mathrm{~m} / \mathrm{s}$, a linear regression model indicates that the VAWR AT reads $0.875 \pm 0.213^{\circ} \mathrm{C}$ above the IMET when the $\mathrm{SW}=900 \mathrm{~W} / \mathrm{m}^{2}$. While the difference decreased with increasing wind speed, even at wind speeds above $10 \mathrm{~m} / \mathrm{s}$, a consistent difference of about $+0.15^{\circ} \mathrm{C}$ occurred when $\mathrm{SW}=900 \mathrm{~W} / \mathrm{m}^{2}$. These results indicate that the IMET sensor and shield combination was less sensitive to solar heating, so the IMET AT series was chosen for subsequent analysis.

\subsubsection{Water Temperature}

Both VAWR and IMET used precision thermistors to measure water temperature. The two WT time series agreed closely over the first 99 days, then exhibited a small drift over the rest of the record. During this initial 99 days, the VAWR and IMET series differed on average by only $0.004 \pm 0.010^{\circ} \mathrm{C}$. Comparison of the two WT series with the temperature record from the SeaCat attached to the discus bridle indicated that the VAWR record had an abrupt temperature shift of about $-0.05^{\circ} \mathrm{C}$ relative to the IMET and SeaCat about May 22, and then appeared to drift by as much as $-0.2^{\circ} \mathrm{C}$ relative to the other records. For these reasons, the IMET WT record was used for subsequent analysis.

\subsubsection{Precipitation}

The IMET supported an R. M. Young self-siphoning rain gauge, which worked by measuring the height of water captured in a vertical column, so that an increase in height indicated rain accumu- 
Air Temperature $\left({ }^{\circ} \mathrm{C}\right)$
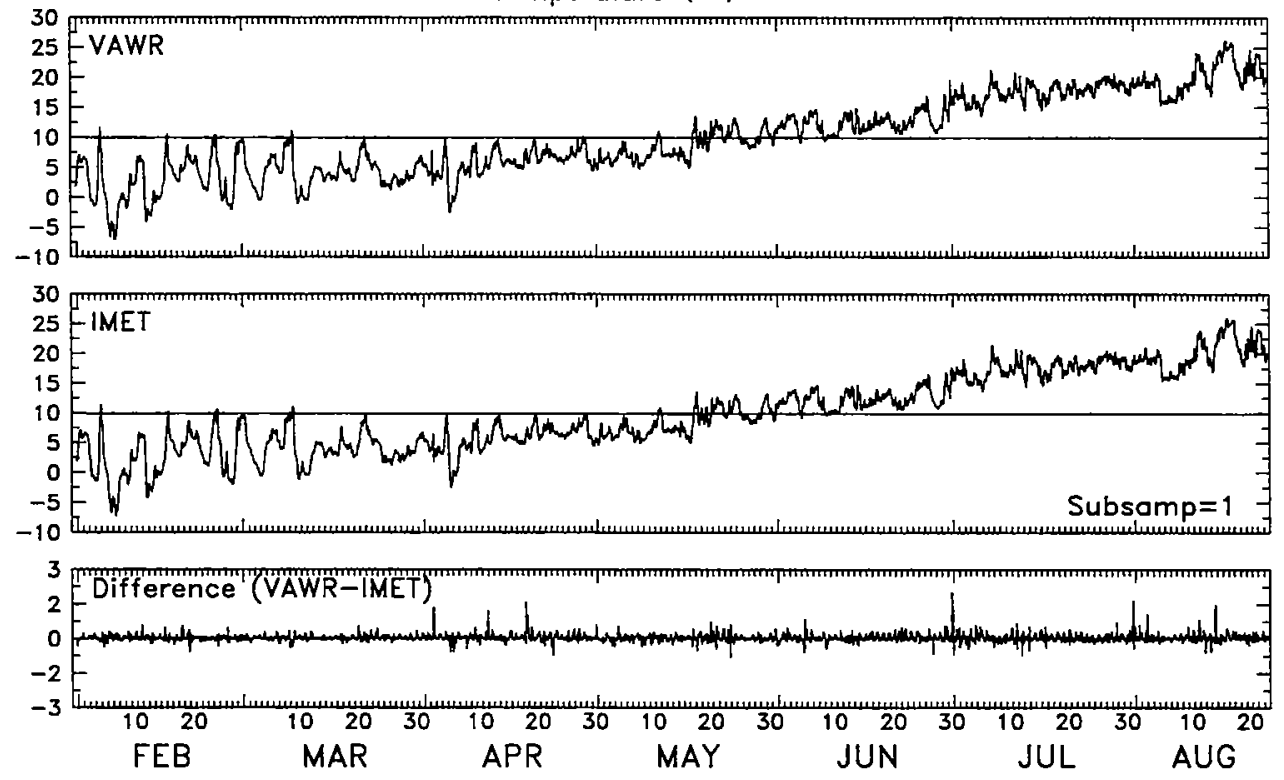

Figure 24: VAWR and IMET Air Temperature and Difference Time Series

Water Temperature $\left({ }^{\circ} \mathrm{C}\right)$
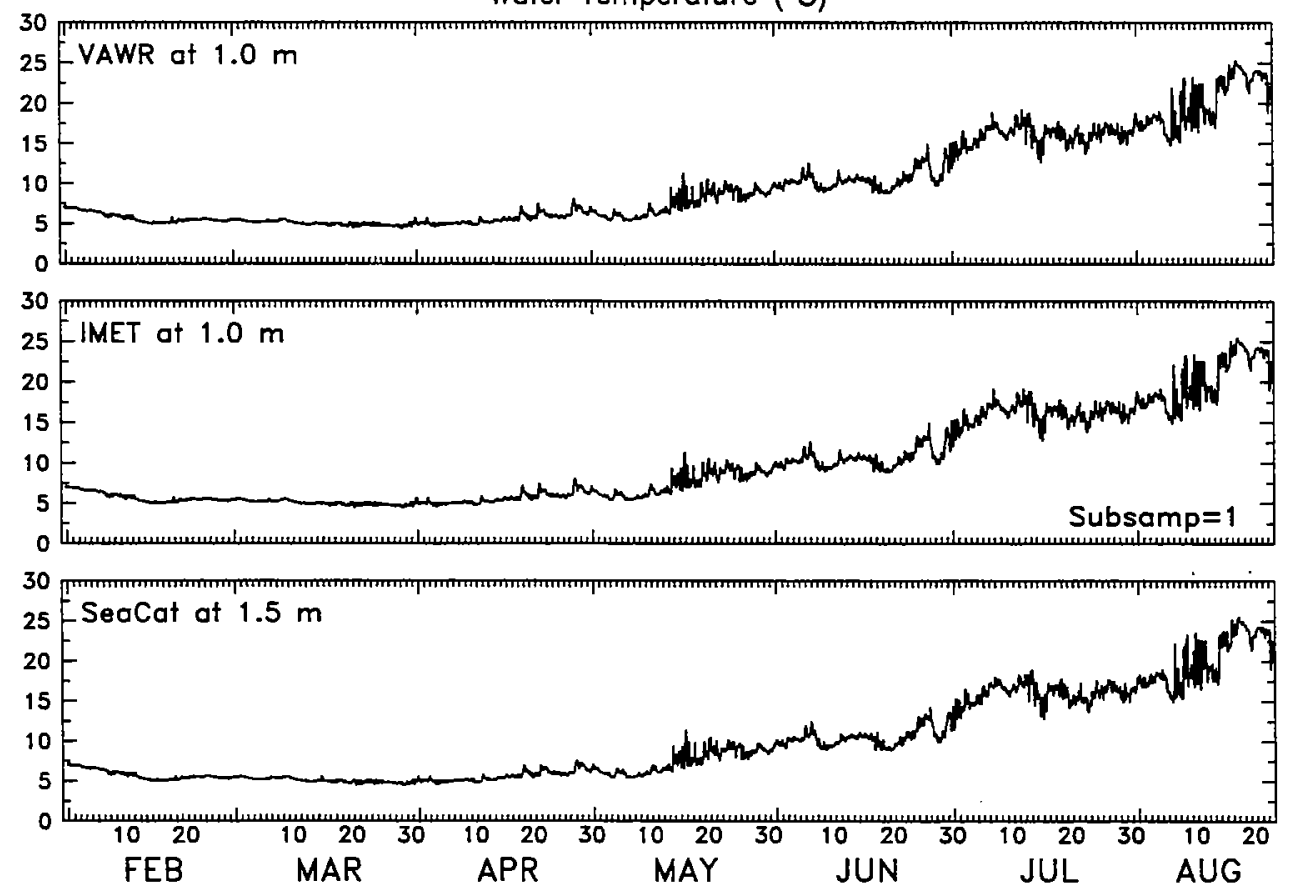

Figure 25: VAWR, IMET and SeaCat Water Temperature Time Series 
Differences in Water Temperature $\left({ }^{\circ} \mathrm{C}\right)$

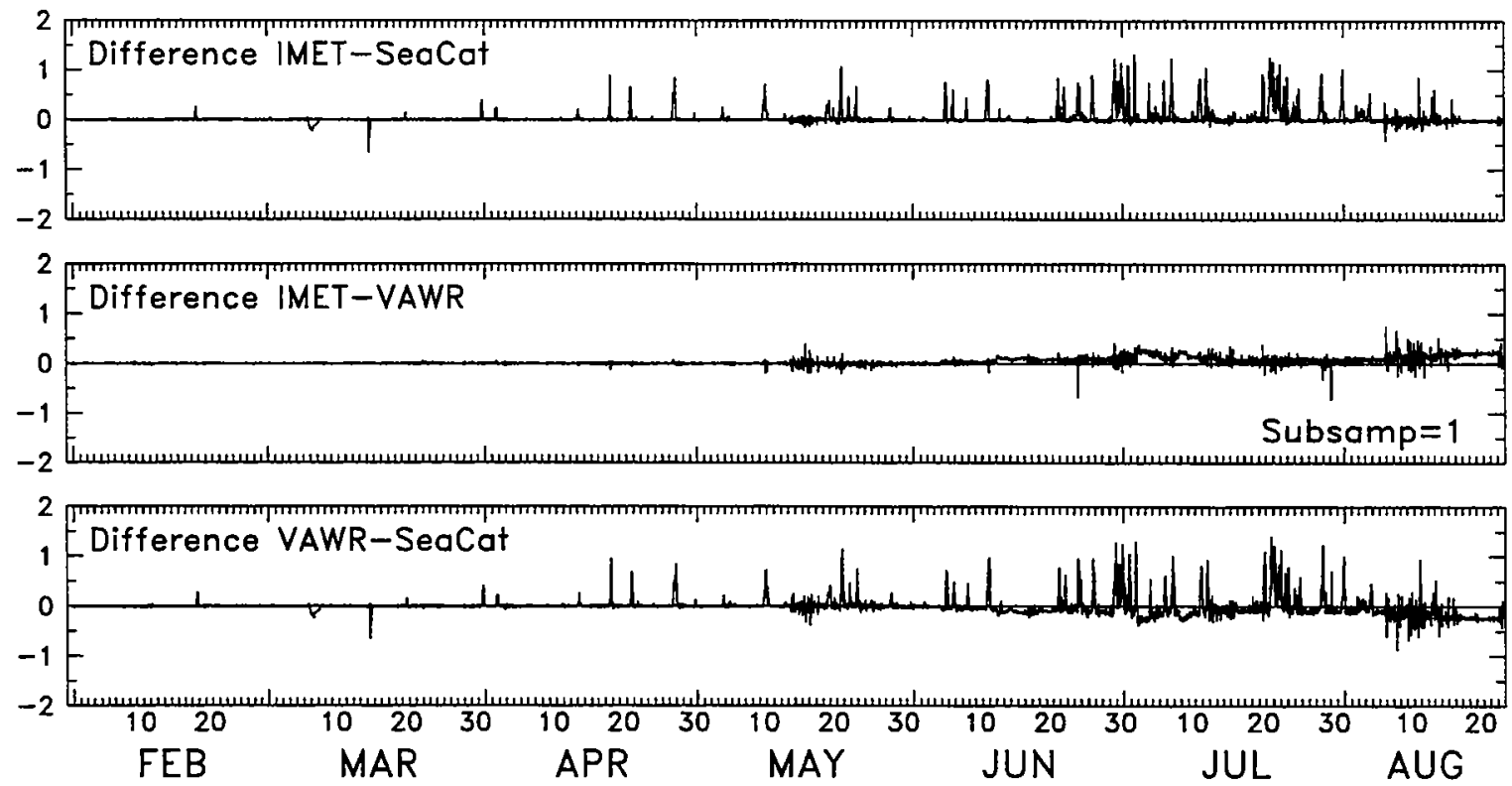

Figure 26: VAWR, IMET, and SeaCat Water Temperature Difference Time Series

lation. When the column became full, the column was drained so the column would be ready to capture new precipitation. The height of the water was measured using electrical capacitance. The gauge electronics were sensitive to the buoy ARGOS transmissions, so that the 1-min precipitation data contained periodic HF spikes roughly every $90 \mathrm{sec}$ that had to be removed before subsequent processing. To reduce these spikes, the 1-minute data was low-pass filtered (with PL64tn, which had a 33-minute half-amplitude period) and used to construct an hourly averaged time series (Figure 27). This record was then corrected for the effects of wind on the catchment efficiency of the gauge. Following the results of Yuter and Parker (Journal of Applied Meteorology, Submitted in 2000), the IMET rain accumulation (RA) data was corrected using equation 7 from Yang et al. (1998),

$$
R A_{(\text {corrected })}=R A_{(\text {measured })} / F
$$

where

$$
F=\exp \left(4.605-0.062 W S^{0.58}\right),
$$

and $W S$ is the wind speed at the height of the mouth of the rain gauge. Here WS was taken as the VAWR wind speed, with no adjustment for the 23-cm difference in sensor heights (Table 5). The corrected rain accumulation time series was used for all subsequent analysis (Figure 27).

\subsubsection{VMCM}

The WHOI Upper Ocean Processes Group did the decoding and preliminary processing of the ST1 WHOI VMCM records. This involved eliminating wild points, checking calibrations, and correcting any time base problems. Final processing was done by S. Lentz (WHOI) and S. Werner (WHOI). 


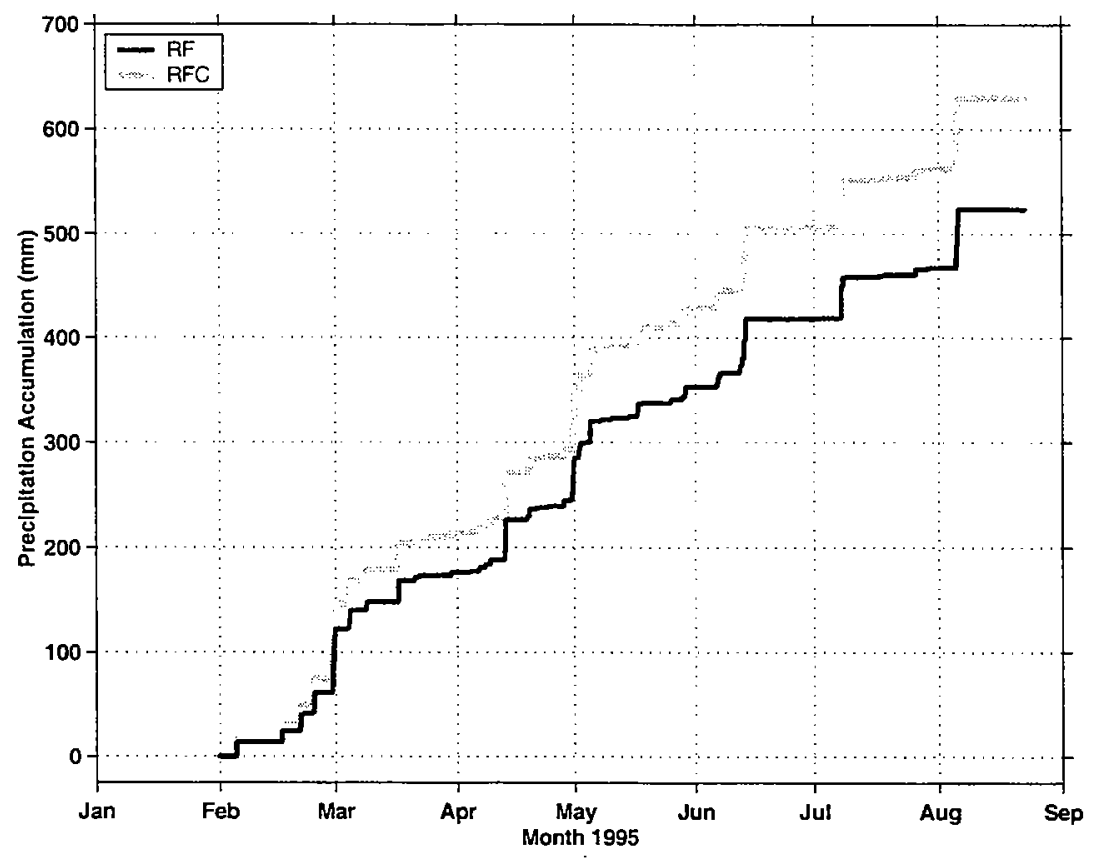

Figure 27: Total Precipitation Accumulation Measured (RF) as Corrected for Wind (RFC)

A comparison of the VMCM and SeaCat temperature data recorded during a stormy winter period when vertical temperature stratification was quite small indicated that several VMCM temperature series could have small bias offsets (less than $0.02^{\circ} \mathrm{C}$ ). These offsets were estimated and the adjusted series are presented here and in the final data set.

\subsubsection{SeaCat}

The ST1 SeaCat basic temperature-conductivity data were decoded using the Sea-Bird software SeaSoft and preliminary editing was done by the WHOI UOP Group. Pre- and post-deployment calibration at Sea-Bird showed insignificant change so that the pre-deployment calibration coefficients were used. As mentioned above, a comparison of VMCM and SeaCat temperature data during a stormy winter period indicated some bias in the VMCM data. To correct for this, the SeaCat data were not changed but bias corrections were made to the VMCM data. The final time series presented here were produced by S. Lentz and S. Werner.

\subsubsection{Brancker TPOD}

The WHOI Brancker TPOD temperature data were decoded using the Brancker software and preliminary editing done by the WHOI UOP Group. The difference in WHOI pre-deployment and post-deployment calibration of the instruments was insignificant. As with the VMCM temperature data, small bias corrections were determined for the TPOD records in comparison to the SeaCat data. These corrections have been applied to the TPOD data presented here. 


\subsubsection{BASS Tripod}

The BASS raw 2-Hz velocity data from each acoustic current sensor were first averaged over the 7.5-minute burst sampling period to produce a burst-averaged velocity value for each half hour. The BASS raw temperature and optical backscatter data were also converted into a burst-average half-hourly time series. J. Fredericks (WHOI) did the initial burst-averaging and S. Werner did all additional processing of the burst-averaged data and produced the final BASS time series presented here.

\subsubsection{Velocity}

The BASS current profiles typically show consistent offsets relative to the best-fit logarithmic profiles. To correct for the offset residuals, Gross (personal communication) developed a procedure in which logarithmic fits are made to the current speeds, and the average residual $U_{\text {res }}$ between the fitted and measured profiles is computed as a function of flow direction. A sine wave $U_{s i n}$ (with non-zero mean) is fitted to $U_{\text {res }}$. The amplitude and orientation of the sine wave give the magnitude and direction of the offset residual.

This procedure was applied to the BASS1 and BASS2 velocity data. Complications arose from flow distortion by the tripod instrumentation, which caused deviations from best-fit logarithmic profiles that could not be attributed to inaccurate sensor velocity zeros (see below). For BASS field data, removal of the computed offset residuals is meaningful only if $U_{\text {sin }}$ represents a good approximation of $U_{\text {res }}$. We chose to remove $U_{s i n}$ from the measurements if the standard deviation of $U_{\text {res }}-U_{s i n}$ was $<0.5 \times\left[U_{\text {sin }}\right]$ averaged over all flow directions, and if the computed offset residuals exceeded $1.0 \mathrm{~cm} / \mathrm{s}$. For BASS1, consistent offsets of $1-1.5 \mathrm{~cm} / \mathrm{s}$ were removed at current meters 1 and 2 . No corrections were applied to BASS2, since offset residuals were $<0.6 \mathrm{~cm} / \mathrm{s}$ at all velocity sensors.

The BASS1 velocity measurements at sensors $1-3$ were corrected for a $13.5^{\circ}$ misalignment error of the sensor axes with respect to sensors 4 and 5 . Misalignment of as much as $\pm 5^{\circ}$ must be expected for both BASS tripods between current sensors 3, 4, and 5. Current sensors 1, 2, and 3 are aligned within $\pm 2^{\circ}$. Compass uncertainties are $\pm 8^{\circ}$ for BASS1 and $\pm 5^{\circ}$ for BASS2.

Flow distortion by the camera and light battery attached to the lowest tripod platform was severe during BASS1. Affected most were measurements at current sensors 1-3 for flow directions 45$85^{\circ}$ clockwise from East. Although not apparent in the velocity record, impacts of flow distortion by the camera and light battery are obvious when computing bottom friction parameters such as shear velocity and bottom roughness length.

Bottom photographs made during BASS1 show that remnants of a plastic bag had wrapped around the lowest two current sensors near the end of the velocity time series (April 15-25). Velocity data and shear velocities from logarithmic fits are noisy during this time and should be interpreted with care.

\subsubsection{Temperature}

Thermal stratification inferred from BASS thermistor data indicated consistent measurement bias offsets as large as $\pm 0.01^{\circ} \mathrm{C}$ for BASS1 and $\pm 0.05^{\circ} \mathrm{C}$ for $\mathrm{BASS} 2\left( \pm 0.5^{\circ} \mathrm{C}\right.$ at $6.02 \mathrm{~m}$ above the 
bottom during BASS2). Corrections were applied as follows: time periods were identified when the near-bottom waters were nearly unstratified, i.e., when the temperature difference between the top and bottom thermistors was "flat." Thermal stratification was computed with respect to the lowest thermistor for each of the identified time periods. The mean temperature difference at each thermistor height was taken as a representative measurement offset of the respective thermistor, and the offset residuals were removed from the thermistor data.

For BASS1, adjustments were based on mean temperature differences between March 3-11. For the more stratified BASS2 deployment, several short periods between July 31 - August 2 (yeardays 212214.5) were chosen for adjustment. Data indicated that this period represents a period of low or no thermal stratification during ebb tide and the first half of flood (that is the temperature difference between thermistors 8 and 1 is mostly "flat"). Short spikes of small thermal stratification (about $0.02^{\circ} \mathrm{C}$ ) occurred during the second half of flood, when Shelf/Slope Front water was advected onbank. Excluding these tidally-induced periods of weak thermal stratification leaves the following periods (yeardays 212.1-212.3, yeardays 212.5-212.8, yeardays 212.9-213.4, yeardays 213.6-213.9, and yeardays 214.1-214.4) to determine thermistor offsets. These offsets were used to produce the final BASS temperature time series presented here.

\subsubsection{Optical Backscatter}

OBSs were placed at five levels on BASS1, but were not part of the BASS2 sensor suite. Processing the BASS1 OBS data began with conversion of the BASS internal OBS record (oscillator counts) to OBS voltage $(0-5 \mathrm{~V}$ range). The next step was aimed at correcting for the small $(\max 0.02 \mathrm{~V})$ clear water offsets of the instruments. This was done by choosing a 10-day segment (February $10-$ 20) over which all OBS records showed no discernible signal. This was, presumably, a clear water portion of the records. Offsets were added to each record so that their means were equivalent over this segment (and equal to the uncorrected mean of the level 3 OBS record).

Typical of optical sensor signals, the OBS voltage records exhibited a gradual drift as the experiment progressed. This was presumably the result of a slow buildup of biological film on the sensors' optical windows. Over the first 41 days of the experiment (to March 15), the sensors were apparently "film free," as the voltage records showed no discernible drift. Two fairly distinct periods of sensor drift followed. The first covered March 15-20 and was marked by a very gradual upward trend of the "base" sensor voltage (e.g., the voltage which appears to underlie any signal). The base voltage from sensor 4 exhibited the first signs of drift. Over this 36-day period, it rose at a rate of roughly $0.005 \mathrm{~V} / \mathrm{d}$. Upward drift of the base voltages of other OBSs also occurred during this period, but began later, near March 31, and was far more gradual, of order $0.002 \mathrm{~V} / \mathrm{d}$. Upward sensor drift during the second period, from April 20 to the record's end, was relatively large, order $0.038 \mathrm{~V} / \mathrm{d}$, at all levels. Large signals also appeared above the rising base voltage of each record during this period. Except for the lowest sensor's record, we believe that these signals were legitimate as they were related to companion temperature and velocity signals. The voltage record from the lowest sensor exhibited unreasonably wild fluctuations (often reaching saturation at $5 \mathrm{~V}$ ) starting on yearday 105. This portion of its record is not shown here. 


\subsubsection{Camera}

The BASS1 camera took one photograph of the bottom (except during strong sediment resuspension events when the bottom was obscured) every eight hours from deployment in February, 1995 through May, 1995. The film was developed and the photographs described in Werner et al.(2001).

\subsection{ST2}

\subsubsection{VMCM and VACM}

The preliminary processing of the ST2 NMFS VMCM and VACM 7.5-minute data was performed by F. Hotchkiss using the standard methods as developed at WHOI (Tarbell et al., 1988). All subsequent processing and the production of the final hourly vector-averaged current, temperature, and light transmission time series presented here were done by J. Manning.

\subsubsection{SeaCat}

The raw temperature-conductivity data from the three ST2 SeaCats were decoded by Manning using the Sea-Bird SeaSoft software. Since the difference in pre-deployment (December 1994) and post-deployment (September 1995) calibration coefficients and sensor drift were insignificant, the post-deployment coefficients were used. The basic 7.5-minute data were first edited and processed (using local NMFS MATLAB routines avg2mtl.m and runave.m) to reformat the basic data and obtain hourly average data, respectively. The reformatting step generates a fractional yearday vector assuming equally spaced samples initiated at 0500 GMT on January 26, 1995 when the instrument was set to begin recording data. The averaging is simply an 8-point mean of the raw data without any weighting with the first hour centered at 2100 GMT on February 2, 1995 after the mooring was deployed.

\subsubsection{Brancker TPOD}

The ST2 Brancker TPOD temperature data were decoded using standard Brancker software and edited by Manning. The difference in WHOI pre-deployment and post-deployment calibration of the instruments was insignificant. The hourly time series were produced by simply extracting every other point of the basic 30-minute series.

\subsubsection{VEMCO Minilog TPOD}

The ST2 Minilog raw temperature data were decoded by Manning using the standard VEMCO minilog.exe routine (version 1.3) and factory generated pre-deployment calibration coefficients. After hand-editing a single spike, the hourly Minilog record was compared to the hourly Brancker TPOD record at the same depth, $5 \mathrm{~m}$. The two records agreed surprisingly well (mean difference less than $0.1^{\circ} \mathrm{C}$ ), considering the low cost of the Minilog. 


\subsection{SF and CR}

Data from the SF and CR moorings (except for the bio-optical systems) were recorded internally on PCMCIA media. These were retrieved from the buoys, and processed using MATLAB routines that checked time and converted the recorded values to temperature, conductivity, etc. All sensors were calibrated each fall, and the pre- and post-cruise calibration constants were checked for drift. No significant changes were observed in any sensor's calibration unless it failed altogether. However, the conductivity sensors and bio-optical sensors also had a non-instrumental drift due to biofouling that was dependent on the season, sensor depth, and previous deployment history.

The buoy times were controlled by a precision internal quartz clock required by the timing of the GOES transmissions, and typically drifted by 5 to 10 seconds in a six-month deployment. The best performance was recorded by the CR buoy with a 10-second drift over one year. The times were compared with the time message received by the GOES satellite, and no corrections to the buoy times were necessary.

\subsubsection{T/C Chain}

For the Sea-Bird temperature and conductivity sensors, in-situ calibrations were attempted by making a time series of CTD profiles by the mooring during the same time that the mooring averaged the in-situ data. However, the temperature sensors typically drifted only a millidegree or two per year, and so were adjusted for mean values with no further time-dependent corrections. The conductivity sensors were fitted with trialkyltin poison cells to reduce biofouling drift, and were corrected by consistency among sensors on a mooring and with the CTD (direct differences between CTD and mooring were as large as the observed drift ( $\sim 0.05$ PSU) in salinity, so were not useful in correcting the salinity time series). The conductivity observations were converted to salinity by the Practical Salinity Scale of 1978 (UNESCO Nos. 36 and 37) and to density by the 1980 Equation of State of Sea Water (UNESCO Nos. 36 and 38). The densities were compared with smooth profile densities from the CTD and among $\mathrm{T} / \mathrm{C}$ pairs on the mooring and the salinities adjusted.

\subsubsection{Bio-Optical System}

The bio-optical data were recorded internally on PCMCIA media. The instruments were started relative to UTC and the recording times checked when the instruments were recovered. Clocks drifted typically less than 1 minute in a six-month deployment, and since the results were averaged to 1-hour time series, no time corrections were made. The data were processed in MATLAB, using the same routines for temperature and conductivity as discussed above. The transmissometer data was checked before and after deployment for transmission in air and with blocked transmission. After recovery, biofouling prevented any real post-deployment calibration or check on sensor drift. The windows were cleaned, and new antifouling rings applied around the windows before each deployment. The fluorometer was treated similarly, with the window cleaned and antifouling protection applied around the window prior to each deployment. The output of the fluorometer was reported as raw voltage output for interpretation by others when compared with biological sampling results. This gives a general qualitative time series of the chlorophyll-a temporal variability. The PAR sensors were treated similarly, by cleaning the diffuser prior to deployment. The 
data was normalized by converting the microamperes current recorded to micromoles $/ \mathrm{sec} / \mathrm{m}^{2}$. A special circuit averaged the PAR sensor output continuously to provide a more realistic measure of the integrated radiation at depth instead of simply using a series of spot measurements. Overflow problems reduced the quality of the PAR observations in the first year.

\subsection{NP}

\subsubsection{RCM}

The raw rate, direction, temperature and conductivity data collected with the three BIO RCMs at NP were decoded and processed at BIO, using standard in-house calibrations for the instruments, to produce hourly records of vector-averaged speed, direction, temperature and salinity. Compass errors were quantified by "swinging" each instrument in a non-magnetic hut located away from the buildings, and conductivity and temperature (thus salinity) were calibrated in regulated baths. Occasional spikes were removed from the time series, based on record mean standard deviation thresholds and/or visual interpretation. The primary data losses occurred in the conductivity records, which were all contaminated by biological fouling at some point during the deployment. The degree of acceptable data was determined by inspection of the salinity records and by the time series of vertical differences in salinity. The final day of good salinity measurements at depths of 23, 37, and $59 \mathrm{~m}$ were judged to be April 8, 1995, November 5, 1994, and December 23, 1994, respectively. In addition, the $\mathrm{RCM}$ at $37 \mathrm{~m}$ had a prolonged period of conductivity spiking in late September, causing the salinity values to be removed.

Stick plots for the hourly and low-passed currents indicated a potential disturbance of the deeper mooring near the beginning of September, reflected by the irregularity of the low-pass currents at all depths, especially near the bottom. A sharp change in the bottom pressure record also occurred around this time.

\subsubsection{TG}

The length of the bottom pressure records was limited by the death of the battery on March 16 , 1995 , but the instrument appeared to function reasonably well prior to that time. The calibrations of the pressure sensor were performed at an in-house facility and included estimates of the temperature sensitivity $\left(0-20^{\circ} \mathrm{C}\right)$ of the Paroscientific quartz crystal transducer. Pre- and postdeployment calibrations were performed on May 31, 1994 and October 2, 1995, and the estimated temperature sensitivities at the in situ temperatures $\left(5-15^{\circ} \mathrm{C}\right)$ and pressures $(<100 \mathrm{dbar})$ ranged from $-0.3 \mathrm{mbar} /{ }^{\circ} \mathrm{C}$ to $+0.3 \mathrm{mbar} /{ }^{\circ} \mathrm{C}$. No corrections for temperature sensitivity have been applied to the pressure data since they appear to be negligible.

The bottom pressure record indicates two occasions on which the mooring may have been disturbed (naturally or unnaturally), namely September 5, 1994 when the temperature and pressure dropped precipitously, and October 4, 1994 when both variables rose significantly and the average pressure seemed to jump by 0.5 dbar or so.

Tidal analysis indicated a significant shift in tidal phase during the last five days of the record. Since this shift did not appear to be consistent with a simple jump in time base, this period was omitted from the final record presented here. 


\subsection{NDBC Environmental Buoys}

The hourly NDBC buoy meteorology data were obtained from the NDBC Marine Environmental Buoy Data base on CD-ROMs. The data files were decoded by C. Alessi and edited by R. Beardsley and Alessi constructed the final time series presented here.

\section{Data Availability on the World Wide Web: http://globec.whoi.edu}

Final best edited versions of the GBSS moored array data presented in this report are available at the U.S. GLOBEC Northeast Atlantic/Georges Bank program website http://globec.whoi.edu. Hourly (or in the case of the BASS data, half-hourly) time series of the GBSS meteorological and oceanographic data spanning the January 31-August 31, 1995 time period stored in MATLAB binary format (.mat) files with self-contained documentation at http://www.whoi.edu/science/PO/ GBSS1995. The BIO RCM and TG records for the full 1994-1995 NP deployment period can. be obtained from the DFO database at http://www.mar.dfo-mpo.gc.ca/sciences/ocean/home.html. Data from the full WHOI 1994-1995 long-term deployments at SF and CR can be obtained at http://kelvin.whoi.edu.

The original basic time series (with some editing but no change in sample interval) for all the GBSS records have been archived at WHOI, and are available on CD-ROM from M. Caurso (mcaurso@whoi.edu). Those who can not handle MATLAB.mat files should contact Mike Caurso for help in obtaining ASCII versions.

\section{1995 GBSS Mooring Cruise Reports}

Table 18 lists the GLOBEC Georges Bank cruise reports related to the 1995 GBSS moored array program. These reports provide detailed information about the related mooring deployment and recovery cruises. Copies of these reports are available from the GLOBEC Georges Bank program office, or accessible on-line at http://globec.whoi.edu/gb/cruise-report-list.

Table 18: 1995 GBSS Mooring Cruise Reports

\begin{tabular}{|c|l|l|l|l|}
\hline Ship/Cruise No. & Start Date & End Date & PI Name & Description \\
\hline PAR94-018 & Jun 24, 1994 & June 30, 1994 & Peter Smith & Mooring \\
EN256 & Oct 26, 1994 & Oct 31, 1994 & Jim Irish & Mooring \\
EN259 & Jan 10, 1995 & Jan 22, 1995 & Ted Durbin & Process \\
EN260 & Jan 29, 1995 & Feb 06, 1995 & Bob Weller & Process \\
EN262 & Feb 23, 1995 & Mar 10, 1995 & Bob Beardsley & Process \\
SJ9504 & Mar 27, 1995 & Apr 04, 1995 & Jim Irish & Mooring \\
SJ9506 & Apr 25, 1995 & May 03, 1995 & Neil Oakey & Process \\
SJ9508 & Jun 06, 1995 & Jun 16, 1995 & Neil Oakey & Process \\
PAR95-010 & Jun 06, 1995 & Jun 13, 1995 & Peter Smith & Mooring \\
EN269 & Jul 10, 1995 & Jul 13, 1995 & Albert Williams & Process \\
EN271 & Aug 22, 1995 & Aug 27, 1995 & Steve Lentz & Process \\
EN274 & Sep 29, 1995 & Oct 05, 1995 & Jim Irish & Mooring \\
\hline
\end{tabular}

$\mathrm{EN}=R / V$ Endeavor, $\quad \mathrm{PAR}=$ C.S.S. Parizeau, $\quad \mathrm{SJ}=R / V$ Seward Johnson 


\section{Basic Statistics of GBSS Moored Array Time Series}

Basic statistics for the hourly averaged data covering the entire time period for each record are shown in Table 19. To facilitate comparisons for temperature and salinity, Tables 20 and 21 show the statistics for the common time period January 31 to August 31, 1995 (GMT). The statistics have been computed for each record for each month in which good data was recorded on at least 15 days. Plots of the monthly means for temperature and salinity values are shown in Figures 28 and 29. Monthly statistics for the NP data are also available from the DFO website given in Section 5 above.

\section{Plots of GBSS Moored Array Time Series}

The data set presented here consists of edited, one-hour-averaged time series of the measured variables. The GBSS moored array time series cover the time period January 31 to August 31, 1995. The current and wind vectors for sites ST1 and ST2 were rotated into an along-bank coordinate system oriented $29^{\circ}$ counter-clockwise with respect to time north so that the along-bank component is positive towards $331^{\circ} \mathrm{T}$ and the cross-bank component is positive towards $61^{\circ} \mathrm{T}$. For examining low-frequency (subtidal) variability, the hourly data were filtered using the PL64 filter. The filter is symmetric with a total of 129 weights. The PL64 filter used has a half-amplitude period of 33 hours and a half-power point of 38 hours. A summary of the PL64 filter, including the generating function, was given by Beardsley et al. (1985). The hourly averaged winds from ST1 VAWR and NDBC 44011 (Georges Bank) and meteorological components are shown in Figures 30-32. Composite (stacked) plots of the PL64 low-pass-filtered current velocity components are shown in Figures 36-40. Composite (stacked) plots of the hourly averaged water temperature and salinity records by mooring are presented in Figures 41-47. Light transmission, BASS OBS data, and bottom pressing fluorometer and attenuation records are presented in Figures 48-50.

Acknowledgments: The authors want to acknowledge the immense expertise and help provided by members of the WHOI Upper Ocean Processes group, the WHOI Rigging Shop and AOPE Mooring Group, the NMFS, USGS, and BIO mooring and data processing groups, and the captains and crews of the research vessels Endeavor, Oceanus, Seward Johnson, and Parizeau. The Stratification Study moored array component proved to be a great success, made possible by the skill and support of many individuals working towards a common goal.

The U.S. GLOBEC Northwest Atlantic/Georges Bank program is jointly funded by the National Science Foundation - Division of Ocean Sciences (NSF) and the NOAA National Marine Fisheries Service (NMFS).

The 1995 Stratification Study moored array field work described in this report was supported by NSF grant OCE 93-13671 (ST1), NSF grant OCE 93-13670 (SF and CR)(Irish). Subsequent data analysis was funded by NSF grant OCE 96-06445. 
Table 19: Statistics of Hourly-Averaged Data

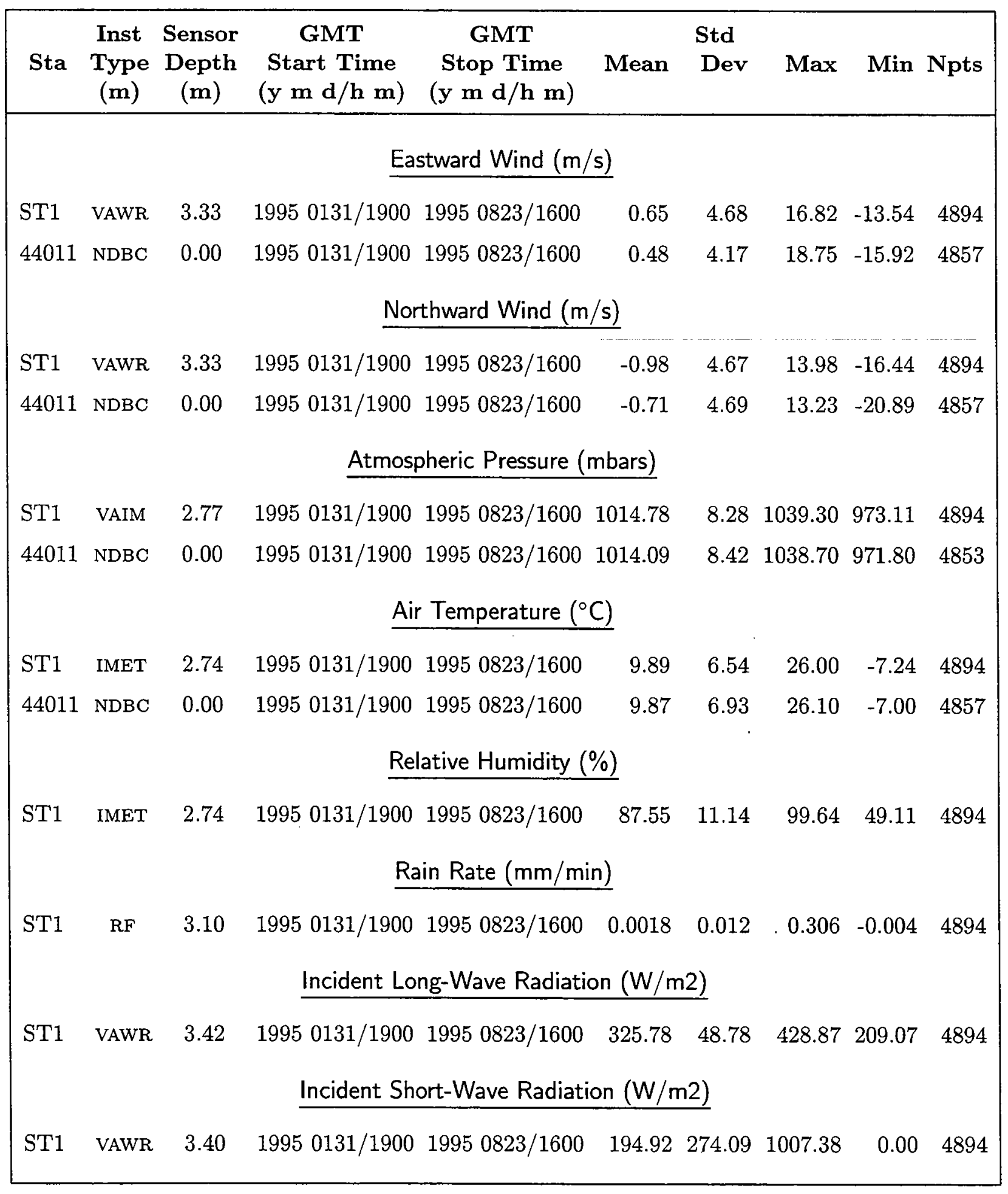


Table 19: Statistics of Hourly-Averaged Data

\begin{tabular}{|c|c|c|c|c|c|c|c|c|c|}
\hline Sta & $\begin{array}{l}\text { Inst } \\
\text { Type } \\
(\mathrm{m})\end{array}$ & $\begin{array}{l}\text { Sensor } \\
\text { Height } \\
\text { (m) }\end{array}$ & $\begin{array}{c}\text { GMT } \\
\text { Start Time } \\
(\mathrm{y} \mathrm{m} \mathrm{d/h} \mathrm{m)}\end{array}$ & $\begin{array}{c}\text { GMT } \\
\text { Stop Time } \\
(\mathrm{y} \mathrm{m} \mathrm{d/h} \mathrm{m)}\end{array}$ & Mean & $\begin{array}{l}\text { Std } \\
\text { Dev }\end{array}$ & $\operatorname{Max}$ & $\operatorname{Min}$ & Npts \\
\hline \multicolumn{10}{|c|}{ Along-Bank Current $(\mathrm{cm} / \mathrm{s})$} \\
\hline ST1 & VMCM & 5.15 & $19950202 / 0000$ & $19950824 / 1600$ & -8.41 & 23.43 & 57.56 & -78.81 & 4889 \\
\hline ST1 & $\mathrm{VMCM}$ & 7.49 & $19950202 / 0000$ & $19950824 / 1600$ & -8.51 & 23.05 & 59.15 & -77.52 & 4889 \\
\hline ST1 & $\mathrm{VMCM}$ & 9.95 & $19950202 / 0000$ & $19950824 / 1600$ & -9.14 & 22.92 & 57.23 & -77.00 & 4889 \\
\hline ST1 & VMCM & 14.00 & $19950202 / 0000$ & $19950824 / 1600$ & -8.60 & 22.26 & 52.90 & -71.64 & 4889 \\
\hline ST1 & VMCM & 19.00 & $19950202 / 0000$ & $19950824 / 1600$ & -8.24 & 22.22 & 45.71 & -67.29 & 4889 \\
\hline ST1 & $\mathrm{VMCM}$ & 25.00 & $19950202 / 0000$ & $19950824 / 1600$ & -10.24 & 22.45 & 44.25 & -70.24 & 4889 \\
\hline ST1 & VMCM & 31.00 & $19950202 / 0000$ & $19950824 / 1600$ & -7.23 & 21.54 & 41.63 & -69.97 & 4889 \\
\hline ST1 & $\mathrm{VMCM}$ & 37.00 & $19950202 / 0000$ & $19950824 / 1600$ & -7.53 & 21.91 & 39.82 & -68.79 & 4889 \\
\hline ST1 & VMCM & 45.50 & $19950202 / 0000$ & $19950824 / 1600$ & -7.62 & 22.09 & 42.17 & -67.16 & 4889 \\
\hline $\mathrm{ST} 1$ & $\mathrm{VMCM}$ & 64.00 & $19950202 / 0000$ & $19950824 / 1600$ & -5.11 & 18.13 & 46.77 & -47.72 & 4889 \\
\hline ST1 & $\mathrm{VMCM}$ & 70.00 & $19950202 / 0000$ & $19950824 / 1600$ & -2.51 & 15.91 & 41.89 & -42.48 & 4889 \\
\hline BS1 & BASS & 72.57 & $19950203 / 1500$ & $19950425 / 0600$ & -2.21 & 16.16 & 37.35 & -39.71 & 1936 \\
\hline BS1 & BASS & 74.47 & $19950203 / 1500$ & 1995 0425/0600 & -3.46 & 14.05 & 31.71 & -36.82 & 1936 \\
\hline $\mathrm{BS} 1$ & BASS & 75.82 & $19950203 / 1500$ & $19950425 / 0600$ & -2.65 & 12.50 & 28.43 & -34.29 & 1936 \\
\hline BS1 & BASS & 76.42 & 1995 0203/1500 & 1995 0425/0600 & -3.40 & 11.54 & 24.21 & -32.11 & 1936 \\
\hline $\mathrm{BS} 1$ & BASS & 76.78 & $19950203 / 1500$ & $19950425 / 0600$ & -2.57 & 10.13 & 22.37 & -29.27 & 1936 \\
\hline $\mathrm{BS} 2$ & BASS & 72.54 & $19950711 / 1500$ & $19950814 / 1300$ & -0.56 & 14.12 & 35.39 & -33.93 & 802 \\
\hline BS2 & BASS & 74.44 & $19950711 / 1500$ & $19950814 / 1300$ & -0.45 & 12.49 & 32.39 & -31.95 & 802 \\
\hline $\mathrm{BS} 2$ & BASS & 75.79 & $19950711 / 1500$ & $19950814 / 1300$ & -0.39 & 11.82 & 28.78 & -29.35 & 802 \\
\hline BS2 & BASS & 76.39 & $19950711 / 1500$ & 1995 0814/1300 & -0.31 & 10.67 & 24.85 & -26.04 & 802 \\
\hline BS2 & BASS & 76.74 & $19950711 / 1500$ & $19950814 / 1300$ & -0.07 & 9.53 & 24.12 & -23.03 & 802 \\
\hline $\mathrm{ST} 2$ & VMCM & 15.00 & $19950203 / 2100$ & $19950517 / 1600$ & -4.07 & 25.08 & 49.58 & -66.91 & 2468 \\
\hline ST2 & VMCM & 35.00 & $19950203 / 2100$ & $19950727 / 1000$ & -5.62 & 22.84 & 43.40 & -60.50 & 4166 \\
\hline $\mathrm{ST} 2$ & VTCT & 62.00 & $19950203 / 1900$ & $19950804 / 2300$ & -2.05 & 18.33 & 38.53 & -44.39 & 4374 \\
\hline NP & $\mathrm{RCM}$ & 23.00 & $19950131 / 0000$ & $19950611 / 2300$ & -1.21 & 42.26 & 79.73 & -79.82 & 3159 \\
\hline NP & $\mathrm{RCM}$ & 37.00 & 1995 0131/0000 & $19950611 / 2300$ & -0.60 & 40.06 & 74.79 & -77.11 & 3159 \\
\hline NP & $\mathrm{RCM}$ & 59.00 & $19950131 / 0000$ & $19950611 / 2300$ & -0.83 & 28.22 & 56.06 & -59.35 & 3159 \\
\hline
\end{tabular}


Table 19: Statistics of Hourly-Averaged Data

\begin{tabular}{|c|c|c|c|c|c|c|c|c|c|}
\hline Sta & $\begin{array}{l}\text { Inst } \\
\text { Type } \\
(\mathbf{m})\end{array}$ & $\begin{array}{l}\text { Sensor } \\
\text { Depth } \\
(\mathbf{m})\end{array}$ & $\begin{array}{c}\text { GMT } \\
\text { Start Time } \\
(\mathbf{y} \mathbf{m ~ d} / \mathbf{h} \mathbf{m})\end{array}$ & $\begin{array}{c}\text { GMT } \\
\text { Stop Time } \\
(\mathbf{y} \mathbf{m} \mathbf{d} / \mathbf{h} \mathbf{m})\end{array}$ & Mean & $\begin{array}{l}\text { Std } \\
\text { Dev }\end{array}$ & $\operatorname{Max}$ & Min & Npts \\
\hline \multicolumn{10}{|c|}{ Cross-Bank Current $(\mathrm{cm} / \mathrm{s})$} \\
\hline ST1 & VMCM & 5.15 & $19950202 / 0000$ & $19950824 / 1600$ & -5.78 & 32.18 & 74.10 & -90.47 & 4889 \\
\hline ST1 & VMCM & 7.49 & $19950202 / 0000$ & $19950824 / 1600$ & -3.48 & 32.53 & 81.45 & -86.26 & 4889 \\
\hline ST1 & VMCM & 9.95 & $19950202 / 0000$ & $19950824 / 1600$ & -2.34 & 32.17 & 81.42 & -84.81 & 4889 \\
\hline ST1 & $\mathrm{VMCM}$ & 14.00 & $19950202 / 0000$ & $19950824 / 1600$ & -2.31 & 31.78 & 70.08 & -83.68 & 4889 \\
\hline $\mathrm{ST} 1$ & $\mathrm{VMCM}$ & 19.00 & $19950202 / 0000$ & $19950824 / 1600$ & -2.13 & 31.61 & 69.44 & -83.68 & 4889 \\
\hline ST1 & VMCM & 25.00 & $19950202 / 0000$ & $19950824 / 1600$ & -1.13 & 31.23 & 67.05 & -77.12 & 4889 \\
\hline ST1 & $\mathrm{VMCM}$ & 31.00 & $19950202 / 0000$ & $19950824 / 1600$ & -1.40 & 31.41 & 68.91 & -80.58 & 4889 \\
\hline ST1 & VMCM & 37.00 & $19950202 / 0000$ & $19950824 / 1600$ & -1.84 & 31.44 & 72.95 & -81.34 & 4889 \\
\hline ST1 & $\mathrm{VMCM}$ & 45.50 & $19950202 / 0000$ & $19950824 / 1600$ & -1.17 & 32.81 & 71.94 & -77.72 & 4889 \\
\hline ST1 & $\mathrm{VMCM}$ & 64.00 & $19950202 / 0000$ & $19950824 / 1600$ & -0.43 & 27.49 & 55.29 & -85.10 & 4889 \\
\hline ST1 & $\mathrm{VMCM}$ & 70.00 & $19950202 / 0000$ & $19950824 / 1600$ & -0.89 & 24.33 & 50.28 & -68.75 & 4889 \\
\hline BS1 & BASS & 72.57 & $19950203 / 1500$ & $19950425 / 0600$ & -2.54 & 22.96 & 40.37 & -49.49 & 1936 \\
\hline BS1 & BASS & 74.47 & $19950203 / 1500$ & $19950425 / 0600$ & -2.43 & 21.24 & 36.40 & -45.95 & 1936 \\
\hline BS1 & BASS & 75.82 & $19950203 / 1500$ & $19950425 / 0600$ & -2.09 & 18.50 & 31.07 & -41.53 & 1936 \\
\hline BS1 & BASS & 76.42 & $19950203 / 1500$ & $19950425 / 0600$ & -0.26 & 16.96 & 30.15 & -36.15 & 1936 \\
\hline BS1 & BASS & 76.78 & 1995 0203/1500 & $19950425 / 0600$ & -1.92 & 14.71 & 26.10 & -33.16 & 1936 \\
\hline BS2 & BASS & 72.54 & $19950711 / 1500$ & $19950814 / 1300$ & 1.11 & 22.90 & 47.11 & -61.98 & 802 \\
\hline BS2 & BASS & 74.44 & $19950711 / 1500$ & $19950814 / 1300$ & 0.76 & 20.76 & 44.58 & -58.21 & 802 \\
\hline BS2 & BASS & 75.79 & $19950711 / 1500$ & $19950814 / 1300$ & 0.48 & 18.51 & 39.74 & -50.37 & 802 \\
\hline $\mathrm{BS} 2$ & BASS & 76.39 & $19950711 / 1500$ & $19950814 / 1300$ & 0.28 & 16.55 & 36.90 & -42.92 & 802 \\
\hline $\mathrm{BS} 2$ & BASS & 76.74 & $19950711 / 1500$ & $19950814 / 1300$ & -0.11 & 14.79 & 31.54 & -41.05 & 802 \\
\hline ST2 & $\mathrm{VMCM}$ & 15.00 & $19950203 / 2100$ & $19950517 / 1600$ & -0.07 & 37.72 & 79.74 & -80.74 & 2468 \\
\hline $\mathrm{ST} 2$ & VMCM & 35.00 & $19950203 / 2100$ & $19950727 / 1000$ & -0.61 & 34.43 & 68.30 & -72.02 & 4166 \\
\hline $\mathrm{ST} 2$ & VTCT & 62.00 & $19950203 / 1900$ & $19950804 / 2300$ & -0.87 & 28.49 & 50.45 & -53.00 & 4374 \\
\hline NP & $\mathrm{RCM}$ & 23.00 & $19950131 / 0000$ & $19950611 / 2300$ & -3.24 & 49.48 & 93.51 & -100.74 & 3159 \\
\hline NP & $\mathrm{RCM}$ & 37.00 & $19950131 / 0000$ & $19950611 / 2300$ & -3.85 & 46.65 & 85.90 & -95.58 & 3159 \\
\hline NP & $\mathrm{RCM}$ & 59.00 & $19950131 / 0000$ & $19950611 / 2300$ & -2.36 & 33.40 & 70.94 & -74.79 & 3159 \\
\hline
\end{tabular}


Table 19: Statistics of Hourly-Averaged Data

\begin{tabular}{|c|c|c|c|c|c|c|c|c|c|}
\hline Sta & $\begin{array}{l}\text { Inst } \\
\text { Type } \\
(\mathbf{m})\end{array}$ & $\begin{array}{c}\text { Sensor } \\
\text { Depth } \\
(\mathrm{m})\end{array}$ & $\begin{array}{c}\text { GMT } \\
\text { Start Time } \\
(\mathbf{y ~} \mathbf{m ~ d} / \mathbf{h} \mathbf{m})\end{array}$ & $\begin{array}{c}\text { GMT } \\
\text { Stop Time } \\
(\mathrm{y} \mathrm{m} \mathrm{d/h} \mathrm{m)}\end{array}$ & Mean & $\begin{array}{l}\text { Std } \\
\text { Dev }\end{array}$ & $\operatorname{Max}$ & Min & Npts \\
\hline \multicolumn{10}{|c|}{ Water Temperature $\left({ }^{\circ} \mathrm{C}\right)$} \\
\hline ST1 & IMET & 1.00 & 1995 0201/0000 & $19950823 / 1600$ & 9.85 & 5.44 & 25.53 & 4.44 & 4889 \\
\hline ST1 & VAWR & 1.50 & $19950201 / 0000$ & $19950823 / 1600$ & 9.80 & 5.41 & 25.51 & 4.43 & 4889 \\
\hline $\mathrm{ST} 1$ & SCAT & 2.00 & $19950201 / 0000$ & $19950823 / 1500$ & 9.75 & 5.37 & 25.47 & 4.42 & 4888 \\
\hline ST1 & TPOD & 3.50 & 1995 0201/0000 & $19950823 / 1600$ & 9.63 & 5.29 & 25.29 & 4.44 & 4889 \\
\hline ST1 & MTR & 5.65 & $19950201 / 0000$ & $19950823 / 1600$ & 9.43 & 5.16 & 24.99 & 4.43 & 4889 \\
\hline ST1 & VMCM & 6.25 & 1995 0201/0000 & $19950823 / 1600$ & 9.40 & 5.13 & 24.97 & 4.44 & 4889 \\
\hline ST1 & SCAT & 7.99 & 1995 0201/0000 & $19950823 / 1600$ & 9.21 & 5.01 & 24.98 & 4.43 & 4889 \\
\hline ST1 & $\mathrm{VMCM}$ & 10.45 & $19950201 / 0000$ & $19950823 / 1600$ & 8.98 & 4.85 & 24.97 & 4.42 & 4889 \\
\hline ST1 & VMCM & 11.05 & 1995 0201/0000 & $19950823 / 1600$ & 8.95 & 4.84 & 24.96 & 4.43 & 4889 \\
\hline ST1 & SCAT & 12.75 & $19950201 / 0000$ & $19950823 / 1600$ & 8.79 & 4.75 & 24.96 & 4.40 & 4889 \\
\hline ST1 & TPOD & 14.50 & 1995 0201/0000 & $19950823 / 1600$ & 8.62 & 4.63 & 24.92 & 4.38 & 4889 \\
\hline ST1 & $\mathrm{VMCM}$ & 19.50 & 1995 0201/0000 & $19950823 / 1600$ & 8.18 & 4.31 & 24.85 & 4.34 & 4889 \\
\hline ST1 & $\mathrm{VMCM}$ & 22.50 & 1995 0201/0000 & $19950823 / 1600$ & 7.97 & 4.14 & 24.82 & 4.32 & 4889 \\
\hline ST1 & TPOD & 25.50 & 1995 0201/0000 & $19950823 / 1600$ & 7.77 & 3.89 & 24.75 & 4.34 & 4889 \\
\hline ST1 & VMCM & 26.10 & 1995 0201/0000 & $19950823 / 1600$ & 7.74 & 3.86 & 24.74 & 4.34 & 4889 \\
\hline ST1 & SCAT & 28.75 & $19950201 / 0000$ & $19950823 / 1600$ & 7.60 & 3.65 & 24.64 & 4.40 & 4889 \\
\hline ST1 & TPOD & 31.50 & $19950201 / 0000$ & $19950823 / 1600$ & 7.47 & 3.40 & 24.52 & 4.40 & 4889 \\
\hline ST1 & VMCM & 37.50 & 1995 0201/0000 & $19950823 / 1600$ & 7.26 & 2.89 & 24.44 & 4.54 & 4889 \\
\hline ST1 & VMCM & 40.75 & 1995 0201/0000 & $19950823 / 1600$ & 7.18 & 2.66 & 24.41 & 4.56 & 4889 \\
\hline ST1 & TPOD & 46.00 & 1995 0201/0000 & $19950823 / 1600$ & 7.08 & 2.32 & 23.49 & 4.62 & 4889 \\
\hline ST1 & VMCM & 46.60 & 1995 0201/0000 & $19950817 / 0400$ & 6.85 & 1.88 & 18.82 & 4.61 & 4733 \\
\hline ST1 & SCAT & 61.50 & $19950201 / 0000$ & $19950823 / 1600$ & 7.04 & 2.05 & 13.77 & 4.61 & 4889 \\
\hline ST1 & TPOD & 64.50 & $19950201 / 0000$ & $19950823 / 1600$ & 7.06 & 2.06 & 13.71 & 4.66 & 4889 \\
\hline ST1 & VMCM & 65.10 & 1995 0201/0000 & $19950823 / 1600$ & 7.06 & 2.07 & 13.68 & 4.66 & 4889 \\
\hline ST1 & SCAT & 67.50 & $19950201 / 0000$ & $19950823 / 1600$ & 7.08 & 2.08 & 13.85 & 4.66 & 4889 \\
\hline
\end{tabular}


Table 19: Statistics of Hourly-Averaged Data

\begin{tabular}{|c|c|c|c|c|c|c|c|c|c|}
\hline Sta & $\begin{array}{c}\text { Inst } \\
\text { Type } \\
(\mathbf{m})\end{array}$ & $\begin{array}{l}\text { Sensor } \\
\text { Depth } \\
(\mathrm{m})\end{array}$ & $\begin{array}{c}\text { GMT } \\
\text { Start Time } \\
(\mathbf{y} \mathbf{m ~ d} / \mathbf{h ~ m})\end{array}$ & $\begin{array}{c}\text { GMT } \\
\text { Stop Time } \\
(\mathbf{y ~ m ~ d / h ~ m ) ~}\end{array}$ & Mean & $\begin{array}{l}\text { Std } \\
\text { Dev }\end{array}$ & $\operatorname{Max}$ & Min & Npts \\
\hline \multicolumn{10}{|c|}{ Water Temperature $\left({ }^{\circ} \mathrm{C}\right)$ (Continued) } \\
\hline $\mathrm{BS} 1$ & BASS & 72.57 & $19950203 / 1500$ & $19950425 / 0600$ & 5.34 & 0.39 & 6.83 & 4.76 & 1936 \\
\hline BS1 & BASS & 73.76 & $19950203 / 1500$ & $19950425 / 0600$ & 5.34 & 0.39 & 6.84 & 4.76 & 1936 \\
\hline BS1 & BASS & 74.47 & $19950203 / 1500$ & $19950425 / 0600$ & 5.34 & 0.39 & 6.83 & 4.76 & 1936 \\
\hline BS1 & BASS & 75.10 & $19950203 / 1500$ & $19950425 / 0600$ & 5.34 & 0.39 & 6.83 & 4.76 & 1936 \\
\hline BS1 & BASS & 75.78 & $19950203 / 1500$ & $19950425 / 0600$ & 5.34 & 0.39 & 6.83 & 4.76 & 1936 \\
\hline BS1 & BASS & 76.38 & $19950203 / 1500$ & $19950425 / 0600$ & 5.34 & 0.39 & 6.85 & 4.76 & 1936 \\
\hline BS1 & BASS & 76.76 & $19950203 / 1500$ & $19950425 / 0600$ & 5.34 & 0.39 & 6.84 & 4.76 & 1936 \\
\hline $\mathrm{BS} 2$ & BASS & 70.98 & $19950711 / 1900$ & $19950824 / 1300$ & 9.55 & 1.38 & 12.65 & 7.89 & 1019 \\
\hline $\mathrm{BS} 2$ & BASS & 72.57 & $19950711 / 1900$ & $19950824 / 1300$ & 9.49 & 1.36 & 12.68 & 7.60 & 993 \\
\hline $\mathrm{BS} 2$ & BASS & 73.52 & $19950711 / 1900$ & $19950824 / 1300$ & 9.55 & 1.38 & 12.65 & 7.90 & 1019 \\
\hline $\mathrm{BS} 2$ & BASS & 74.47 & $19950711 / 1900$ & $19950824 / 1300$ & 9.55 & 1.39 & 12.66 & 7.88 & 1019 \\
\hline $\mathrm{BS} 2$ & BASS & 75.18 & $19950711 / 1900$ & $19950824 / 1300$ & 9.56 & 1.38 & 12.66 & 7.93 & 1019 \\
\hline $\mathrm{BS} 2$ & BASS & 75.82 & $19950711 / 1900$ & $19950824 / 1300$ & 9.55 & 1.39 & 12.66 & 7.89 & 1019 \\
\hline $\mathrm{BS} 2$ & BASS & 76.42 & $19950711 / 1900$ & $19950824 / 1300$ & 9.54 & 1.39 & 12.66 & 7.88 & 1019 \\
\hline $\mathrm{BS} 2$ & BASS & 76.68 & $19950711 / 1900$ & $19950824 / 1300$ & 9.55 & 1.39 & 12.67 & 7.88 & 1019 \\
\hline ST2 & SCAT & 1.00 & $19950203 / 2100$ & $19950804 / 2300$ & 8.53 & 4.05 & 18.65 & 4.67 & 4371 \\
\hline ST2 & TPOD & 5.40 & $19950203 / 2100$ & $19950804 / 2300$ & 7.98 & 3.35 & 17.97 & 4.67 & 4371 \\
\hline ST2 & SCAT & 11.00 & $19950203 / 2100$ & $19950804 / 2300$ & 7.50 & 2.70 & 16.91 & 4.67 & 4371 \\
\hline ST2 & VMCM & 17.00 & $19950203 / 2100$ & $19950517 / 1600$ & 5.44 & 0.51 & 9.94 & 4.67 & 2468 \\
\hline ST2 & TPOD & 26.10 & $19950203 / 2100$ & $19950804 / 2300$ & 6.84 & 1.88 & 14.74 & 4.68 & 4371 \\
\hline ST2 & SCAT & 32.00 & $19950203 / 2100$ & $19950804 / 2300$ & 6.80 & 1.81 & 11.73 & 4.67 & 4371 \\
\hline ST2 & $\mathrm{VMCM}$ & 37.70 & $19950203 / 2100$ & $19950727 / 1000$ & 6.60 & 1.61 & 10.95 & 4.67 & 4166 \\
\hline ST2 & TPOD & 42.80 & $19950203 / 2100$ & $19950804 / 2300$ & 6.72 & 1.70 & 11.47 & 4.67 & 4371 \\
\hline ST2 & VACM & 61.50 & $19950204 / 0000$ & $19950804 / 2300$ & 6.72 & 1.67 & 11.13 & 4.65 & 4368 \\
\hline
\end{tabular}


Table 19: Statistics of Hourly-Averaged Data

\begin{tabular}{|c|c|c|c|c|c|c|c|c|c|}
\hline Sta & $\begin{array}{l}\text { Inst } \\
\text { Type } \\
(\mathrm{m})\end{array}$ & $\begin{array}{c}\text { Sensor } \\
\text { Depth } \\
(\mathrm{m})\end{array}$ & $\begin{array}{c}\text { GMT } \\
\text { Start Time } \\
(\text { y m d/h m) }\end{array}$ & $\begin{array}{c}\text { GMT } \\
\text { Stop Time } \\
(\mathbf{y ~} \mathbf{m ~ d} / \mathbf{h ~ m})\end{array}$ & Mean & $\begin{array}{l}\text { Std } \\
\text { Dev }\end{array}$ & $\operatorname{Max}$ & Min & Npts \\
\hline \multicolumn{10}{|c|}{ Water Temperature $\left({ }^{\circ} \mathrm{C}\right)$ (Continued) } \\
\hline $\mathrm{CR}$ & SBTC & 2.00 & $19950402 / 0400$ & $19950823 / 1600$ & 10.48 & 3.79 & 17.28 & 5.23 & 3445 \\
\hline $\mathrm{CR}$ & SBTC & 10.00 & $19950402 / 0400$ & $19950823 / 1600$ & 10.45 & 3.79 & 17.10 & 5.22 & 3445 \\
\hline $\mathrm{SF}$ & SBTC & 1.00 & $19950201 / 0000$ & $19950823 / 1600$ & 11.05 & 4.90 & 25.82 & 5.14 & 3619 \\
\hline $\mathrm{SF}$ & SBTC & 5.00 & 1995 0201/0000 & $19950823 / 1600$ & 11.38 & 4.43 & 24.32 & 5.23 & 3096 \\
\hline $\mathrm{SF}$ & SBTC & 10.00 & $19950201 / 0000$ & $19950823 / 1600$ & 9.93 & 4.34 & 24.17 & 5.13 & 3619 \\
\hline $\mathrm{SF}$ & SBTC & 15.00 & $19950201 / 0000$ & $19950823 / 1600$ & 9.36 & 4.09 & 24.20 & 5.10 & 3619 \\
\hline SF & SBTC & 20.00 & 1995 0201/0000 & $19950823 / 1600$ & 8.81 & 3.68 & 23.48 & 5.12 & 3619 \\
\hline SF & SBTC & 25.00 & $19950201 / 0000$ & $19950823 / 1600$ & 8.38 & 3.20 & 22.91 & 5.08 & 3619 \\
\hline $\mathrm{SF}$ & SBTC & 30.00 & $19950201 / 0000$ & $19950823 / 1600$ & 8.07 & 2.75 & 22.74 & 4.98 & 3619 \\
\hline SF & SBTC & 35.00 & 1995 0201/0000 & $19950823 / 1600$ & 7.88 & 2.38 & 21.83 & 4.96 & 3619 \\
\hline $\mathrm{SF}$ & SBTC & 40.00 & $19950201 / 0000$ & $19950304 / 1500$ & 5.72 & 0.49 & 6.81 & 5.11 & 760 \\
\hline SF & SBTC & 45.00 & $19950201 / 0000$ & $19950823 / 1600$ & 7.72 & 1.99 & .15 .31 & 5.00 & 3619 \\
\hline $\mathrm{SF}$ & SBTC & 50.00 & $19950201 / 0000$ & $19950823 / 1600$ & 7.78 & 1.91 & 13.45 & 4.99 & 3499 \\
\hline $\mathrm{SF}$ & PMEL & 1.00 & $19950401 / 1900$ & $19950823 / 1600$ & 11.18 & 4.94 & 25.76 & 4.49 & 3459 \\
\hline $\mathrm{SF}$ & PMEL & 10.00 & $19950401 / 1900$ & $19950823 / 1600$ & 10.14 & 4.48 & 24.23 & 4.45 & 3459 \\
\hline $\mathrm{SF}$ & PMEL & 20.00 & $19950401 / 1900$ & $19950823 / 1600$ & 8.79 & 3.74 & 23.49 & 4.37 & 3459 \\
\hline $\mathrm{SF}$ & PMEL & 50.00 & $19950401 / 1900$ & $19950823 / 1600$ & 7.71 & 2.22 & 20.23 & 4.37 & 3459 \\
\hline NP & $\mathrm{RCM}$ & 23.00 & $19950102 / 0000$ & $19950611 / 2300$ & 5.76 & 1.05 & 8.11 & 3.52 & 3855 \\
\hline NP & $\mathrm{RCM}$ & 37.00 & $19950102 / 0000$ & $19950611 / 2300$ & 5.77 & 1.04 & 8.12 & 4.18 & 3855 \\
\hline NP & $\mathrm{RCM}$ & 59.00 & $19950102 / 0000$ & $19950611 / 2300$ & 5.78 & 1.04 & 8.14 & 4.25 & 3855 \\
\hline NP & TG & 73.00 & $19950101 / 0000$ & 1995 0310/2300 & 6.17 & 1.19 & 8.14 & 4.52 & 1655 \\
\hline
\end{tabular}


Table 20: Monthly Hourly-Averaged Statistics

\begin{tabular}{|c|c|c|c|c|c|c|c|c|c|}
\hline Sta & $\begin{array}{l}\text { Inst } \\
\text { Type } \\
(\mathrm{m})\end{array}$ & $\begin{array}{l}\text { Sensor } \\
\text { Height } \\
\text { (m) }\end{array}$ & $\begin{array}{c}\text { GMT } \\
\text { Start Time } \\
(\mathrm{y} \mathrm{m} \mathrm{d/h} \mathrm{m)}\end{array}$ & $\begin{array}{c}\text { GMT } \\
\text { Stop Time } \\
(\mathbf{y ~ m ~ d / h ~ m ) ~}\end{array}$ & Mean & $\begin{array}{l}\text { Std } \\
\text { Dev }\end{array}$ & Max & Min & Npts \\
\hline \multicolumn{10}{|c|}{ February 1995 Water Temperature $\left({ }^{\circ} \mathrm{C}\right)$} \\
\hline ST1 & IMET & 1.00 & $19950201 / 0000$ & $19950228 / 2300$ & 5.76 & 0.59 & 7.16 & 4.97 & 672 \\
\hline ST1 & VAWR & 1.50 & $19950201 / 0000$ & $19950228 / 2300$ & 5.76 & 0.59 & 7.17 & 4.97 & 672 \\
\hline ST1 & SCAT & 2.00 & $19950201 / 0000$ & $19950228 / 2300$ & 5.76 & 0.59 & 7.17 & 4.96 & 672 \\
\hline ST1 & TPOD & 3.50 & $19950201 / 0000$ & $19950228 / 2300$ & 5.76 & 0.60 & 7.16 & 4.98 & 672 \\
\hline ST1 & MTR & 5.65 & $19950201 / 0000$ & $19950228 / 2300$ & 5.76 & 0.60 & 7.17 & 4.97 & 672 \\
\hline ST1 & VMCM & 6.25 & $19950201 / 0000$ & $19950228 / 2300$ & 5.76 & 0.60 & 7.17 & 4.98 & 672 \\
\hline ST1 & SCAT & 7.99 & $19950201 / 0000$ & $19950228 / 2300$ & 5.75 & 0.60 & 7.17 & 4.97 & 672 \\
\hline $\mathrm{ST} 1$ & VMCM & 10.45 & $19950201 / 0000$ & $19950228 / 2300$ & 5.75 & 0.60 & 7.17 & 4.97 & 672 \\
\hline ST1 & VMCM & 11.05 & $19950201 / 0000$ & $19950228 / 2300$ & 5.75 & 0.60 & 7.17 & 4.98 & 672 \\
\hline ST1 & SCAT & 12.75 & $19950201 / 0000$ & $19950228 / 2300$ & 5.75 & 0.60 & 7.17 & 4.98 & 672 \\
\hline ST1 & TPOD & 14.50 & 1995 0201/0000 & $228 / 2300$ & 5.75 & 0.60 & 7.17 & 4.98 & 672 \\
\hline ST1 & VMCM & 19.50 & $19950201 / 0000$ & $19950228 / 2300$ & 5.75 & 0.60 & 7.15 & 4.98 & 672 \\
\hline ST1 & VMCM & 22.50 & $19950201 / 0000$ & $19950228 / 2300$ & 5.74 & 0.61 & 7.16 & 4.98 & 672 \\
\hline ST1 & TPOD & 25.50 & 1995 0201/0000 & $19950228 / 2300$ & 5.74 & 0.61 & 7.10 & 4.98 & 672 \\
\hline ST1 & VMCM & 26.10 & $19950201 / 0000$ & $19950228 / 2300$ & 5.74 & 0.61 & & & 672 \\
\hline ST1 & SCAT & 28.75 & $19950201 / 0000$ & $19950228 / 2300$ & 5.74 & 0.60 & 7.09 & 4.98 & 672 \\
\hline ST1 & TPOD & 31.50 & $19950201 / 0000$ & $19950228 / 2300$ & 5.74 & 0.61 & 7.08 & 4.98 & 672 \\
\hline ST1 & $\mathrm{VMCM}$ & 37.50 & $19950201 / 0000$ & $19950228 / 2300$ & 5.73 & 0.61 & 7.07 & 4.98 & 672 \\
\hline ST1 & $\mathrm{VMCM}$ & 40.75 & $19950201 / 0000$ & $19950228 / 2300$ & 5.73 & 0.61 & 7.07 & 4.98 & 672 \\
\hline ST1 & TPOD & 46.00 & $19950201 / 0000$ & $19950228 / 2300$ & 5.73 & 0.61 & 7.06 & & 672 \\
\hline ST1 & VMCM & 46.60 & $19950201 / 0000$ & $228 / 2300$ & 5.73 & 0.61 & 7.06 & 4.98 & 672 \\
\hline ST1 & SCAT & 61.50 & $19950201 / 0000$ & $19950228 / 2300$ & 5.72 & 0.61 & 7.02 & 4.98 & 672 \\
\hline ST1 & TPOD & 64.50 & 1995 0201/0000 & $19950228 / 2300$ & 5.72 & 0.61 & 7.02 & 4.99 & 672 \\
\hline ST1 & VMCM & 65.10 & $19950201 / 0000$ & $19950228 / 2300$ & 5.72 & 0.61 & 7.02 & 4.99 & 672 \\
\hline ST1 & SCAT & 67.50 & $19950201 / 0000$ & $19950228 / 2300$ & 5.72 & 0.61 & 7.02 & 4.98 & 672 \\
\hline $\mathrm{BS} 1$ & BASS & 72.57 & $19950203 / 1500$ & $19950228 / 2348$ & 5.69 & & & & 610 \\
\hline BS1 & BASS & 73.76 & $0203 / 1500$ & $19950228 / 2348$ & 5.69 & 0.49 & 6.84 & 5.08 & 610 \\
\hline BS1 & BASS & 74.47 & $19950203 / 1500$ & $19950228 / 2348$ & 5.69 & 0.49 & 6.83 & 5.09 & 610 \\
\hline $\mathrm{BS} 1$ & BASS & 75.10 & $19950203 / 1500$ & $19950228 / 2348$ & 5.69 & 0.48 & 6.83 & & 610 \\
\hline BS1 & BASS & 75.78 & $19950203 / 1500$ & $19950228 / 2348$ & 5.69 & 0.49 & 6.83 & 5.08 & 610 \\
\hline $\mathrm{BS} 1$ & BASS & 76.38 & $19950203 / 1500$ & $19950228 / 2348$ & 5.69 & 0.49 & 6.85 & 5.09 & 610 \\
\hline BS1 & BASS & 76.76 & $19950203 / 1500$ & $19950228 / 2348$ & 5.69 & 0.49 & 6.84 & 5.08 & 610 \\
\hline $\mathrm{ST} 2$ & SCAT & & $19950203 / 2100$ & $19950228 / 2300$ & 5.37 & 0.45 & 6.67 & 4.76 & 603 \\
\hline $\mathrm{ST} 2$ & TPOD & 5.40 & $19950203 / 2100$ & $19950228 / 2300$ & 5.37 . & 0.45 & 6.65 & 4.76 & 603 \\
\hline $\mathrm{ST} 2$ & SCAT & 11.00 & $19950203 / 2100$ & $19950228 / 2300$ & 5.37 & 0.46 & 6.68 & 4.77 & 603 \\
\hline ST2 & VMCM & 17.00 & $19950203 / 2100$ & $19950228 / 2300$ & 5.37 & 0.46 & 6.70 & 4.77 & 603 \\
\hline \multirow[t]{2}{*}{$\mathrm{ST} 2$} & TPOD & 26.10 & $19950203 / 2100$ & $19950228 / 2300$ & 5.36 & 0.46 & 6.65 & 4.78 & 603 \\
\hline & & & & & & & & & \\
\hline
\end{tabular}


Table 20: Monthly Hourly-Averaged Statistics

\begin{tabular}{|c|c|c|c|c|c|c|c|c|c|}
\hline Sta & $\begin{array}{l}\text { Inst } \\
\text { Type } \\
(\mathbf{m})\end{array}$ & $\begin{array}{l}\text { Sensor } \\
\text { Height } \\
\quad(\mathbf{m})\end{array}$ & $\begin{array}{c}\text { GMT } \\
\text { Start Time } \\
(\mathbf{y} \mathbf{m ~ d} / \mathbf{h ~ m})\end{array}$ & $\begin{array}{c}\text { GMT } \\
\text { Stop Time } \\
(\mathbf{y ~} \mathbf{~ d} / \mathbf{h ~ m})\end{array}$ & Mean & $\begin{array}{l}\text { Std } \\
\text { Dev }\end{array}$ & $\operatorname{Max}$ & Min & Npts \\
\hline \multicolumn{10}{|c|}{ February 1995 Water Temperature $\left({ }^{\circ} \mathrm{C}\right)$ (Continued) } \\
\hline ST2 & SCAT & 32.00 & $19950203 / 2100$ & $19950228 / 2300$ & 5.37 & 0.46 & 6.67 & 4.77 & 603 \\
\hline $\mathrm{ST} 2$ & VMCM & 37.70 & $19950203 / 2100$ & 1995 0228/2300 & 5.36 & 0.46 & 6.68 & 4.77 & 603 \\
\hline ST2 & TPOD & 42.80 & $19950203 / 2100$ & $19950228 / 2300$ & 5.36 & 0.46 & 6.63 & 4.77 & 603 \\
\hline $\mathrm{ST} 2$ & VACM & 61.50 & $19950204 / 0000$ & $19950228 / 2300$ & 5.34 & 0.46 & 6.62 & 4.75 & 600 \\
\hline CR & SBTC & 2.00 & - & - & - & - & - & - & - \\
\hline CR & SBTC & 10.00 & - & - & - & - & - & - & - \\
\hline $\mathrm{SF}$ & SBTC & 1.00 & 1995 0201/0000 & $19950228 / 2300$ & 5.80 & 0.48 & 6.80 & 5.14 & 672 \\
\hline SF & SBTC & 5.00 & $19950201 / 0000$ & 1995 0210/2300 & 6.36 & 0.34 & 6.79 & 5.46 & 237 \\
\hline SF & SBTC & 10.00 & $19950201 / 0000$ & $19950228 / 2300$ & 5.77 & 0.49 & 6.79 & 5.13 & 672 \\
\hline $\mathrm{SF}$ & SBTC & 15.00 & $19950201 / 0000$ & $19950228 / 2300$ & 5.77 & 0.49 & 6.79 & 5.14 & 672 \\
\hline SF & SBTC & 20.00 & $19950201 / 0000$ & $19950228 / 2300$ & 5.77 & 0.49 & 6.79 & 5.12 & 672 \\
\hline $\mathrm{SF}$ & SBTC & 25.00 & $19950201 / 0000$ & $19950228 / 2300$ & 5.77 & 0.49 & 6.80 & 5.12 & 672 \\
\hline SF & SBTC & 30.00 & 1995 0201/0000 & $19950228 / 2300$ & 5.77 & 0.50 & 6.80 & 5.12 & 672 \\
\hline $\mathrm{SF}$ & SBTC & 35.00 & $19950201 / 0000$ & $19950228 / 2300$ & 5.78 & 0.50 & 6.80 & 5.11 & 672 \\
\hline $\mathrm{SF}$ & SBTC & 40.00 & $19950201 / 0000$ & $19950228 / 2300$ & 5.77 & 0.50 & 6.81 & 5.11 & 672 \\
\hline SF & SBTC & 45.00 & $19950201 / 0000$ & $19950228 / 2300$ & 5.78 & 0.50 & 6.83 & 5.12 & 672 \\
\hline $\mathrm{SF}$ & SBTC & 50.00 & $19950201 / 0000$ & $19950227 / 1500$ & 5.81 & 0.50 & 6.83 & 5.12 & 640 \\
\hline $\mathrm{SF}$ & PMEL & 1.00 & - & - & - & - & - & - & - \\
\hline $\mathrm{SF}$ & PMEL & 10.00 & - & - & - & - & - & - & - \\
\hline SF & PMEL & 20.00 & - & - & - & - & - & - & - \\
\hline $\mathrm{SF}$ & PMEL & 50.00 & - & - & - & - & - & - & - \\
\hline NP & $\mathrm{RCM}$ & 23.00 & $19950201 / 0000$ & $19950228 / 2300$ & 5.46 & 0.66 & 6.68 & 4.58 & 672 \\
\hline NP & $\mathrm{RCM}$ & 37.00 & $19950201 / 0000$ & $19950228 / 2300$ & 5.47 & 0.66 & 6.68 & 4.58 & 672 \\
\hline NP & $\mathrm{RCM}$ & 59.00 & $19950201 / 0000$ & $19950228 / 2300$ & 5.49 & 0.66 & 6.70 & 4.58 & 672 \\
\hline NP & TG & 73.00 & $19950201 / 0000$ & $19950228 / 2300$ & 5.38 & 0.65 & 6.68 & 4.52 & 672 \\
\hline \multicolumn{10}{|c|}{ March 1995 Water Temperature $\left({ }^{\circ} \mathrm{C}\right)$} \\
\hline ST1 & IMET & 1.00 & 1995 0301/0000 & $19950331 / 2300$ & 5.05 & 0.27 & 5.73 & 4.44 & 744 \\
\hline ST1 & VAWR & 1.50 & $19950301 / 0000$ & $19950331 / 2300$ & 5.05 & 0.27 & 5.62 & 4.43 & 744 \\
\hline ST1 & SCAT & 2.00 & $19950301 / 0000$ & $19950331 / 2300$ & 5.04 & 0.27 & 5.62 & 4.42 & 744 \\
\hline ST1 & TPOD & 3.50 & $19950301 / 0000$ & $19950331 / 2300$ & 5.04 & 0.26 & 5.62 & 4.44 & 744 \\
\hline $\mathrm{ST} 1$ & MTR & 5.65 & 1995 0301/0000 & $19950331 / 2300$ & 5.04 & 0.26 & 5.62 & 4.43 & 744 \\
\hline ST1 & $\mathrm{VMCM}$ & 6.25 & $19950301 / 0000$ & $19950331 / 2300$ & 5.04 & 0.26 & 5.62 & 4.44 & 744 \\
\hline ST1 & SCAT & 7.99 & $19950301 / 0000$ & $19950331 / 2300$ & 5.03 & 0.26 & 5.62 & 4.43 & 744 \\
\hline \multicolumn{10}{|c|}{ March 1995 Statistics Continued on Next Page } \\
\hline
\end{tabular}


Table 20: Monthly Hourly-Averaged Statistics

\begin{tabular}{|c|c|c|c|c|c|c|c|c|c|}
\hline Sta & $\begin{array}{l}\text { Inst } \\
\text { Type } \\
(\mathbf{m})\end{array}$ & $\begin{array}{c}\text { Sensor } \\
\text { Height } \\
\quad(\mathbf{m})\end{array}$ & $\begin{array}{c}\text { GMT } \\
\text { Start Time } \\
(\mathbf{y ~} \mathrm{m} \mathrm{d} / \mathbf{h} \mathbf{m})\end{array}$ & $\begin{array}{c}\text { GMT } \\
\text { Stop Time } \\
(\mathbf{y ~ m ~ d / h ~ m ) ~}\end{array}$ & Mean & $\begin{array}{l}\text { Std } \\
\text { Dev }\end{array}$ & $\operatorname{Max}$ & Min & Npts \\
\hline \multicolumn{10}{|c|}{ March 1995 Water Temperature $\left({ }^{\circ} \mathrm{C}\right)$ (Continued) } \\
\hline ST1 & VMCM & 10.45 & 1995 0301/0000 & $19950331 / 2300$ & 5.03 & 0.26 & 5.61 & 4.42 & 744 \\
\hline ST1 & VMCM & 11.05 & $19950301 / 0000$ & $19950331 / 2300$ & 5.03 & 0.26 & 5.60 & 4.43 & 744 \\
\hline ST1 & SCAT & 12.75 & $19950301 / 0000$ & $19950331 / 2300$ & 5.02 & 0.26 & 5.58 & 4.40 & 744 \\
\hline ST1 & TPOD & 14.50 & $19950301 / 0000$ & $19950331 / 2300$ & 5.02 & 0.26 & 5.58 & 4.38 & 744 \\
\hline ST1 & VMCM & 19.50 & $19950301 / 0000$ & $19950331 / 2300$ & 5.01 & 0.26 & 5.59 & 4.34 & 744 \\
\hline ST1 & VMCM & 22.50 & $19950301 / 0000$ & $19950331 / 2300$ & 5.01 & 0.26 & 5.60 & 4.32 & 744 \\
\hline ST1 & TPOD & 25.50 & $19950301 / 0000$ & $19950331 / 2300$ & 5.02 & 0.25 & 5.56 & 4.34 & 744 \\
\hline ST1 & VMCM & 26.10 & $19950301 / 0000$ & $19950331 / 2300$ & 5.02 & 0.25 & 5.55 & 4.34 & 744 \\
\hline ST1 & SCAT & 28.75 & $19950301 / 0000$ & $19950331 / 2300$ & 5.03 & 0.24 & 5.53 & 4.41 & 744 \\
\hline ST1 & TPOD & 31.50 & $19950301 / 0000$ & $19950331 / 2300$ & 5.04 & 0.22 & 5.51 & 4.40 & 744 \\
\hline ST1 & VMCM & 37.50 & $19950301 / 0000$ & $19950331 / 2300$ & 5.06 & 0.20 & 5.50 & 4.57 & 744 \\
\hline ST1 & VMCM & 40.75 & $19950301 / 0000$ & $19950331 / 2300$ & 5.07 & 0.20 & 5.49 & 4.62 & 744 \\
\hline ST1 & TPOD & 46.00 & $19950301 / 0000$ & $19950331 / 2300$ & 5.08 & 0.19 & 5.49 & 4.65 & 744 \\
\hline ST1 & VMCM & 46.60 & $19950301 / 0000$ & $19950331 / 2300$ & 5.08 & 0.19 & 5.49 & 4.65 & 744 \\
\hline ST1 & SCAT & 61.50 & $19950301 / 0000$ & $19950331 / 2300$ & 5.08 & 0.19 & 5.49 & 4.66 & 744 \\
\hline ST1 & TPOD & 64.50 & $19950301 / 0000$ & $19950331 / 2300$ & 5.08 & 0.19 & 5.49 & 4.66 & 744 \\
\hline ST1 & VMCM & 65.10 & $19950301 / 0000$ & $19950331 / 2300$ & 5.08 & 0.19 & 5.49 & 4.66 & 744 \\
\hline ST1 & SCAT & 67.50 & $19950301 / 0000$ & $19950331 / 2300$ & 5.08 & 0.19 & 5.49 & 4.66 & 744 \\
\hline BS1 & BASS & 72.57 & $19950301 / 1500$ & $19950331 / 2300$ & 5.18 & 0.19 & 5.58 & 4.76 & 744 \\
\hline BS1 & BASS & 73.76 & $19950301 / 1500$ & $19950331 / 2300$ & 5.17 & 0.19 & 5.58 & 4.76 & 744 \\
\hline BS1 & BASS & 74.47 & $19950301 / 1500$ & $19950331 / 2300$ & 5.18 & 0.18 & 5.58 & 4.76 & 744 \\
\hline BS1 & BASS & 75.10 & $19950301 / 1500$ & $19950331 / 2300$ & 5.18 & 0.19 & 5.57 & 4.76 & 744 \\
\hline $\mathrm{BS} 1$ & BASS & 75.78 & $19950301 / 1500$ & $19950331 / 2300$ & 5.18 & 0.19 & 5.58 & 4.76 & 744 \\
\hline $\mathrm{BS} 1$ & BASS & 76.38 & $19950301 / 1500$ & $19950331 / 2300$ & 5.18 & 0.19 & 5.58 & 4.76 & 744 \\
\hline BS1 & BASS & 76.76 & $19950301 / 1500$ & $19950331 / 2300$ & 5.18 & 0.19 & 5.58 & 4.76 & 744 \\
\hline ST2 & SCAT & 1.00 & 1995 0301/0000 & $19950331 / 2300$ & 5.14 & 0.16 & 5.62 & 4.67 & 744 \\
\hline ST2 & TPOD & 5.40 & $19950301 / 0000$ & $19950331 / 2300$ & 5.12 & 0.15 & 5.60 & 4.67 & 744 \\
\hline ST2 & SCAT & 11.00 & $19950301 / 0000$ & $19950331 / 2300$ & 5.12 & 0.15 & 5.49 & 4.67 & 744 \\
\hline ST2 & $\mathrm{VMCM}$ & 17.00 & $19950301 / 0000$ & $19950331 / 2300$ & 5.10 & 0.14 & 5.48 & 4.67 & 744 \\
\hline ST2 & TPOD & 26.10 & $19950301 / 0000$ & $19950331 / 2300$ & 5.10 & 0.13 & 5.46 & 4.68 & 744 \\
\hline ST2 & SCAT & 32.00 & $19950301 / 0000$ & $19950331 / 2300$ & 5.10 & 0.13 & 5.46 & 4.67 & 744 \\
\hline ST2 & $\mathrm{VMCM}$ & 37.70 & $19950301 / 0000$ & $19950331 / 2300$ & 5.10 & 0.13 & 5.46 & 4.67 & 744 \\
\hline ST2 & TPOD & 42.80 & $19950301 / 0000$ & $19950331 / 2300$ & 5.10 & 0.13 & 5.45 & 4.67 & 744 \\
\hline ST2 & VACM & 61.50 & $19950301 / 0000$ & $19950331 / 2300$ & 5.09 . & 0.13 & 5.43 & 4.65 & 744 \\
\hline $\mathrm{CR}$ & SBTC & 2.00 & 一 & - & - & - & - & - & - \\
\hline \multirow[t]{2}{*}{ CR } & SBTC & 10.00 & - & - & - & - & - & - & - \\
\hline & \multicolumn{9}{|c|}{ March 1995 Statistics Continued on Next Page } \\
\hline
\end{tabular}


Table 20: Monthly Hourly-Averaged Statistics

\begin{tabular}{|c|c|c|c|c|c|c|c|c|c|}
\hline Sta & $\begin{array}{l}\text { Inst } \\
\text { Type } \\
(\mathbf{m})\end{array}$ & $\begin{array}{l}\text { Sensor } \\
\text { Height } \\
\quad(\mathrm{m})\end{array}$ & $\begin{array}{c}\text { GMT } \\
\text { Start Time } \\
(\mathbf{y} \mathbf{m ~ d} / \mathbf{h ~ m})\end{array}$ & $\begin{array}{c}\text { GMT } \\
\text { Stop Time } \\
(\mathbf{y ~ m ~ d / h ~ m ) ~}\end{array}$ & Mean & $\begin{array}{l}\text { Std } \\
\text { Dev }\end{array}$ & $\operatorname{Max}$ & Min & Npts \\
\hline \multicolumn{10}{|c|}{ March 1995 Water Temperature $\left({ }^{\circ} \mathrm{C}\right)$ (Continued) } \\
\hline $\mathrm{SF}$ & SBTC & 1.00 & $19950301 / 0000$ & $19950304 / 1500$ & 5.34 & 0.08 & 5.50 & 5.18 & 88 \\
\hline $\mathrm{SF}$ & SBTC & 5.00 & - & - & - & - & 一 & - & - \\
\hline $\mathrm{SF}$ & SBTC & 10.00 & $19950301 / 0000$ & $19950304 / 1500$ & 5.32 & 0.09 & 5.48 & 5.17 & 88 \\
\hline $\mathrm{SF}$ & SBTC & 15.00 & $19950301 / 0000$ & $19950304 / 1500$ & 5.32 & 0.09 & 5.48 & 5.16 & 88 \\
\hline $\mathrm{SF}$ & SBTC & 20.00 & $19950301 / 0000$ & $19950304 / 1500$ & 5.33 & 0.09 & 5.47 & 5.17 & 88 \\
\hline $\mathrm{SF}$ & SBTC & 25.00 & $19950301 / 0000$ & $19950304 / 1500$ & 5.33 & 0.09 & 5.46 & 5.17 & 88 \\
\hline $\mathrm{SF}$ & SBTC & 30.00 & $19950301 / 0000$ & $19950304 / 1500$ & 5.33 & 0.09 & 5.46 & 5.17 & 88 \\
\hline $\mathrm{SF}$ & SBTC & 35.00 & $19950301 / 0000$ & $19950304 / 1500$ & 5.32 & 0.09 & 5.46 & 5.16 & 88 \\
\hline $\mathrm{SF}$ & SBTC & 40.00 & $19950301 / 0000$ & $19950304 / 1500$ & 5.32 & 0.09 & 5.46 & 5.17 & 88 \\
\hline $\mathrm{SF}$ & SBTC & 45.00 & $19950301 / 0000$ & $19950304 / 1500$ & 5.33 & 0.08 & 5.46 & 5.17 & 88 \\
\hline SF & SBTC & 50.00 & - & - & - & - & - & - & - \\
\hline SF & pmel & 1.00 & - & - & - & - & - & - & - \\
\hline SF & pmel & 10.00 & - & - & 一 & $\therefore$ & - & - & - \\
\hline $\mathrm{SF}$ & pmel & 20.00 & - & - & - & - & - & - & - \\
\hline $\mathrm{SF}$ & pmel & 50.00 & - & - & - & - & - & - & - \\
\hline NP & $\mathrm{rcm}$ & 23.00 & $19950301 / 0000$ & $19950331 / 2300$ & 4.84 & 0.16 & 5.19 & 4.51 & 744 \\
\hline NP & $\mathrm{rcm}$ & 37.00 & $19950301 / 0000$ & $19950331 / 2300$ & 4.85 & 0.16 & 5.19 & 4.56 & 744 \\
\hline NP & $\mathrm{rcm}$ & 59.00 & $19950301 / 0000$ & $19950331 / 2300$ & 4.87 & 0.16 & 5.21 & 4.56 & 744 \\
\hline NP & TG & 73.00 & $19950301 / 0000$ & $19950310 / 2300$ & 4.72 & 0.08 & 4.96 & 4.55 & 239 \\
\hline \multicolumn{10}{|c|}{ April 1995 Water Temperature $\left({ }^{\circ} \mathrm{C}\right)$} \\
\hline ST1 & IMET & 1.00 & $19950401 / 0000$ & $19950430 / 2300$ & 5.65 & 0.69 & 8.07 & 4.63 & 720 \\
\hline ST1 & VAWR & 1.50 & 1995 0401/0000 & $19950430 / 2300$ & 5.63 & 0.65 & 7.55 & 4.61 & 720 \\
\hline ST1 & SCAT & 2.00 & $19950401 / 0000$ & $19950430 / 2300$ & 5.61 & 0.62 & 7.42 & 4.62 & 720 \\
\hline ST1 & TPOD & 3.50 & $19950401 / 0000$ & $19950430 / 2300$ & 5.57 & 0.57 & 7.24 & 4.62 & 720 \\
\hline ST1 & MTR & 5.65 & $19950401 / 0000$ & $19950430 / 2300$ & 5.52 & 0.52 & 7.16 & 4.63 & 720 \\
\hline ST1 & VMCM & 6.25 & $19950401 / 0000$ & $19950430 / 2300$ & 5.51 & 0.52 & 7.15 & 4.62 & 720 \\
\hline ST1 & SCAT & 7.99 & $19950401 / 0000$ & $19950430 / 2300$ & 5.47 & 0.49 & 6.79. & 4.63 & 720 \\
\hline ST1 & $\mathrm{VMCM}$ & 10.45 & $19950401 / 0000$ & $19950430 / 2300$ & 5.42 & 0.45 & 6.64 & 4.63 & 720 \\
\hline ST1 & VMCM & 11.05 & $19950401 / 0000$ & $19950430 / 2300$ & 5.42 & 0.45 & 6.62 & 4.60 & 720 \\
\hline ST1 & SCAT & 12.75 & $19950401 / 0000$ & $19950430 / 2300$ & 5.38 & 0.43 & 6.63 & 4.60 & 720 \\
\hline STI & TPOD & 14.50 & $19950401 / 0000$ & $19950430 / 2300$ & 5.35 & 0.41 & 6.65 & 4.62 & 720 \\
\hline ST1 & VMCM & 19.50 & $19950401 / 0000$ & $19950430 / 2300$ & 5.27 & 0.36 & 6.45 & 4.64 & 720 \\
\hline ST1 & $\mathrm{VMCM}$ & 22.50 & $19950401 / 0000$ & $19950430 / 2300$ & 5.23 & 0.35 & 6.23 & 4.43 & 720 \\
\hline ST1 & TPOD & 25.50 & $19950401 / 0000$ & $19950430 / 2300$ & 5.21 & 0.34 & 6.15 & 4.35 & 720 \\
\hline \multicolumn{10}{|c|}{ April 1995 Statistics Continued on Next Page } \\
\hline
\end{tabular}


Table 20: Monthly Hourly-Averaged Statistics

\begin{tabular}{|c|c|c|c|c|c|c|c|c|c|}
\hline Sta & $\begin{array}{l}\text { Inst } \\
\text { Type } \\
(\mathbf{m})\end{array}$ & $\begin{array}{l}\text { Sensor } \\
\text { Height } \\
\text { (m) }\end{array}$ & $\begin{array}{c}\text { GMT } \\
\text { Start Time } \\
(y \mathrm{~m} \mathrm{~d} / \mathrm{h} \mathrm{m})\end{array}$ & $\begin{array}{c}\text { GMT } \\
\text { Stop Time } \\
(y \mathbf{m ~ d} / \mathbf{h ~ m})\end{array}$ & Mean & $\begin{array}{l}\text { Std } \\
\text { Dev }\end{array}$ & Max & Min & Npts \\
\hline \multicolumn{10}{|c|}{ April 1995 Water Temperature $\left({ }^{\circ} \mathrm{C}\right)$ (Continued) } \\
\hline ST1 & VMCM & 26.10 & $19950401 / 0000$ & $19950430 / 2300$ & 5.21 & 0.34 & 6.12 & 4.36 & 720 \\
\hline ST1 & SCAT & 28.75 & $19950401 / 0000$ & $19950430 / 2300$ & 5.19 & 0.33 & 6.08 & 4.40 & 720 \\
\hline ST1 & TPOD & 31.50 & $19950401 / 0000$ & $19950430 / 2300$ & 5.18 & 0.33 & 6.07 & 4.47 & 720 \\
\hline ST1 & VMCM & 37.50 & 1995 0401/0000 & $19950430 / 2300$ & 5.17 & 0.32 & 6.06 & 4.54 & 720 \\
\hline ST1 & VMCM & 40.75 & $19950401 / 0000$ & $19950430 / 2300$ & 5.17 & 0.32 & 6.06 & 4.56 & 720 \\
\hline ST1 & TPOD & 46.00 & $19950401 / 0000$ & $19950430 / 2300$ & 5.17 & 0.32 & 6.07 & 4.62 & 720 \\
\hline ST1 & VMCM & 46.60 & $19950401 / 0000$ & $19950430 / 2300$ & 5.17 & 0.32 & 6.06 & 4.61 & 720 \\
\hline ST1 & SCAT & 61.50 & $19950401 / 0000$ & $19950430 / 2300$ & 5.20 & 0.30 & 6.05 & 4.75 & 720 \\
\hline ST1 & TPOD & 64.50 & 1995 0401/0000 & $19950430 / 2300$ & 5.20 & 0.30 & 6.05 & 4.76 & 720 \\
\hline ST1 & VMCM & 65.10 & $19950401 / 0000$ & $19950430 / 2300$ & 5.20 & 0.30 & 6.05 & 4.76 & 720 \\
\hline ST1 & SCAT & 67.50 & $19950401 / 0000$ & $19950430 / 2300$ & 5.20 & 0.30 & 6.04 & 4.75 & 720 \\
\hline $\mathrm{BS} 1$ & BASS & 72.57 & $19950401 / 1500$ & $19950425 / 0600$ & 5.18 & 0.19 & 5.62 & 4.86 & 582 \\
\hline $\mathrm{BS} 1$ & BASS & 73.76 & $19950401 / 1500$ & $19950425 / 0600$ & 5.18 & 0.19 & 5.62 & 4.85 & 582 \\
\hline $\mathrm{BS} 1$ & BASS & 74.47 & $19950401 / 1500$ & $19950425 / 0600$ & 5.18 & 0.19 & 5.62 & 4.86 & 582 \\
\hline $\mathrm{BS} 1$ & BASS & 75.10 & $19950401 / 1500$ & 1995 0425/0600 & 5.18 & 0.19 & 5.62 & 4.86 & 582 \\
\hline BS1 & BASS & 75.78 & $19950401 / 1500$ & $19950425 / 0600$ & 5.18 & 0.19 & 5.62 & 4.86 & 582 \\
\hline $\mathrm{BS} 1$ & BASS & 76.38 & $19950401 / 1500$ & $19950425 / 0600$ & 5.18 & 0.19 & 5.62 & 4.86 & 582 \\
\hline BS1 & BASS & 76.76 & $19950401 / 1500$ & $19950425 / 0600$ & 5.18 & 0.19 & 5.62 & 4.86 & 582 \\
\hline ST2 & SCAT & 1.00 & $19950401 / 0000$ & $19950430 / 2300$ & 5.61 & 0.46 & 7.55 & 4.96 & 720 \\
\hline ST2 & TPOD & 5.40 & $19950401 / 0000$ & $19950430 / 2300$ & 5.51 & 0.35 & 6.75 & 4.95 & 720 \\
\hline $\mathrm{ST} 2$ & SCAT & 11.00 & $19950401 / 0000$ & $19950430 / 2300$ & 5.46 & 0.32 & 6.39 & 4.97 & 720 \\
\hline ST2 & $\mathrm{VMCM}$ & 17.00 & $19950401 / 0000$ & $19950430 / 2300$ & 5.42 & 0.31 & 6.35 & 4.95 & 720 \\
\hline ST2 & TPOD & 26.10 & $19950401 / 0000$ & $19950430 / 2300$ & 5.39 & 0.31 & 6.31 & 4.94 & 720 \\
\hline ST2 & SCAT & 32.00 & 1995 0401/0000 & $19950430 / 2300$ & 5.38 & 0.32 & 6.33 & 4.94 & 720 \\
\hline ST2 & $\mathrm{VMCM}$ & 37.70 & $19950401 / 0000$ & $19950430 / 2300$ & 5.37 & 0.32 & 6.30 & 4.94 & 720 \\
\hline ST2 & TPOD & 42.80 & 1995 0401/0000 & $19950430 / 2300$ & 5.36 & 0.32 & 6.26 & 4.94 & 720 \\
\hline $\mathrm{ST} 2$ & VACM & 61.50 & 1995 0401/0000 & $19950430 / 2300$ & 5.35 & 0.32 & 6.25 & 4.93 & 720 \\
\hline CR & SBTC & 2.00 & $19950402 / 0400$ & $19950430 / 2300$ & 5.86 & 0.46 & 6.95 & 5.23 & 692 \\
\hline CR & SBTC & 10.00 & $19950402 / 0400$ & $19950430 / 2300$ & 5.84 & 0.46 & 6.84 & 5.22 & 692 \\
\hline SF & SBTC & 1.00 & $19950426 / 1900$ & $19950430 / 2300$ & 6.61 & 0.45 & 7.82 & 5.92 & 106 \\
\hline $\mathrm{SF}$ & SBTC & 5.00 & $19950426 / 1900$ & $19950430 / 2300$ & 6.26 & 0.25 & 7.03 & 5.87 & 106 \\
\hline $\mathrm{SF}$ & SBTC & 10.00 & $19950426 / 1900$ & $19950430 / 2300$ & 6.05 & 0.28 & 6.73 & 5.42 & 106 \\
\hline $\mathrm{SF}$ & SBTC & 15.00 & $19950426 / 1900$ & $19950430 / 2300$ & 5.85 & 0.38 & 6.64 & 5.10 & 106 \\
\hline $\mathrm{SF}$ & SBTC & 20.00 & $19950426 / 1900$ & $19950430 / 2300$ & 5.69 & 0.29 & 6.64 & 5.21 & 106 \\
\hline $\mathrm{SF}$ & SBTC & 25.00 & $19950426 / 1900$ & $19950430 / 2300$ & 5.56 & 0.22 & 6.52 & 5.08 & 106 \\
\hline \multirow[t]{2}{*}{$\mathrm{SF}$} & SBTC & 30.00 & $19950426 / 1900$ & $19950430 / 2300$ & 5.51 & 0.18 & 5.72 & 5.07 & 106 \\
\hline & \multicolumn{9}{|c|}{ April 1995 Statistics Continued on Next Page? } \\
\hline
\end{tabular}


Table 20: Monthly Hourly-Averaged Statistics

\begin{tabular}{|c|c|c|c|c|c|c|c|c|c|}
\hline Sta & $\begin{array}{l}\text { Inst } \\
\text { Type } \\
(\mathbf{m})\end{array}$ & $\begin{array}{l}\text { Sensor } \\
\text { Height } \\
\quad(\mathbf{m})\end{array}$ & $\begin{array}{c}\text { GMT } \\
\text { Start Time } \\
(y \mathrm{~m} \mathrm{~d} / \mathrm{h} \mathrm{m})\end{array}$ & $\begin{array}{c}\text { GMT } \\
\text { Stop Time } \\
(\mathbf{y ~ m ~ d / h ~ m ) ~}\end{array}$ & Mean & $\begin{array}{l}\text { Std } \\
\text { Dev }\end{array}$ & $\operatorname{Max}$ & Min & Npts \\
\hline \multicolumn{10}{|c|}{ April 1995 Water Temperature $\left({ }^{\circ} \mathrm{C}\right)$ (Continued) } \\
\hline SF & SBTC & 35.00 & $19950426 / 1900$ & $19950430 / 2300$ & 5.50 & 0.19 & 5.69 & 5.06 & 106 \\
\hline SF & $\mathrm{SBTC}$ & 40.00 & - & - & - & - & - & - & - \\
\hline $\mathrm{SF}$ & SBTC & 45.00 & $19950426 / 1900$ & $19950430 / 2300$ & 5.51 & 0.19 & 5.69 & 5.07 & 106 \\
\hline SF & SBTC & 50.00 & $19950426 / 1900$ & $19950430 / 2300$ & 5.50 & 0.19 & 5.69 & 5.07 & 106 \\
\hline $\mathrm{SF}$ & PMEL & 1.00 & $19950401 / 1900$ & $19950430 / 2300$ & 5.49 & 0.68 & 7.63 & 4.49 & 706 \\
\hline $\mathrm{SF}$ & PMEL & 10.00 & $19950401 / 1900$ & $19950430 / 2300$ & 5.34 & 0.50 & 6.75 & 4.45 & 706 \\
\hline $\mathrm{SF}$ & PMEL & 20.00 & $19950401 / 1900$ & $19950430 / 2300$ & 5.17 & 0.40 & 6.65 & 4.37 & 706 \\
\hline $\mathrm{SF}$ & PMEL & 50.00 & $19950401 / 1900$ & $19950430 / 2300$ & 5.05 & 0.36 & 5.67 & 4.37 & 706 \\
\hline NP & $\mathrm{RCM}$ & 23.00 & $19950401 / 0000$ & $19950430 / 2300$ & 4.75 & 0.34 & 5.43 & 3.52 & 720 \\
\hline NP & $\mathrm{RCM}$ & 37.00 & $19950401 / 0000$ & $19950430 / 2300$ & 4.78 & 0.30 & 5.44 & 4.18 & 720 \\
\hline NP & $\mathrm{RCM}$ & 59.00 & $19950401 / 0000$ & $19950430 / 2300$ & 4.79 & 0.29 & 5.46 & 4.25 & 720 \\
\hline NP & TG & 73.00 & - & - & - & - & - & - & - \\
\hline \multicolumn{10}{|c|}{ May 1995 Water Temperature $\left({ }^{\circ} \mathrm{C}\right)$} \\
\hline ST1 & IMET & 1.00 & $19950501 / 0000$ & $19950531 / 2300$ & 7.56 & 1.47 & 11.31 & 5.41 & 744 \\
\hline ST1 & VAWR & 1.50 & $19950501 / 0000$ & $19950531 / 2300$ & 7.53 & 1.46 & 11.41 & 5.40 & 744 \\
\hline ST1 & SCAT & 2.00 & $19950501 / 0000$ & $19950531 / 2300$ & 7.49 & 1.43 & 11.36 & 5.40 & 744 \\
\hline ST1 & TPOD & 3.50 & $19950501 / 0000$ & $19950531 / 2300$ & 7.40 & 1.40 & 11.42 & 5.40 & 744 \\
\hline ST1 & MTR & 5.65 & $19950501 / 0000$ & $19950531 / 2300$ & 7.28 & 1.36 & 11.59 & 5.34 & 744 \\
\hline ST1 & $\mathrm{VMCM}$ & 6.25 & $19950501 / 0000$ & $19950531 / 2300$ & 7.26 & 1.35 & 11.62 & 5.31 & 744 \\
\hline ST1 & SCAT & 7.99 & $19950501 / 0000$ & $19950531 / 2300$ & 7.18 & 1.34 & 11.85 & 5.28 & 744 \\
\hline ST1 & $\mathrm{VMCM}$ & 10.45 & $19950501 / 0000$ & $19950531 / 2300$ & 7.09 & 1.34 & 11.92 & 5.13 & 744 \\
\hline ST1 & $\mathrm{VMCM}$ & 11.05 & $19950501 / 0000$ & $19950531 / 2300$ & 7.08 & 1.34 & 11.89 & 5.12 & 744 \\
\hline ST1 & SCAT & 12.75 & $19950501 / 0000$ & $19950531 / 2300$ & 7.04 & 1.34 & 11.95 & 5.09 & 744 \\
\hline ST1 & TPOD & 14.50 & $19950501 / 0000$ & $19950531 / 2300$ & 6.99 & 1.34 & 11.98 & 5.08 & 744 \\
\hline ST1 & $\mathrm{VMCM}$ & 19.50 & $19950501 / 0000$ & $19950531 / 2300$ & 6.92 & 1.39 & 12.06 & 5.09 & 744 \\
\hline ST1 & $\mathrm{VMCM}$ & 22.50 & $19950501 / 0000$ & $19950531 / 2300$ & 6.92 & 1.46 & 12.08 & 5.09 & 744 \\
\hline ST1 & TPOD & 25.50 & $19950501 / 0000$ & $19950531 / 2300$ & 6.95 & 1.54 & 12.10 & 5.09 & 744 \\
\hline ST1 & VMCM & 26.10 & $19950501 / 0000$ & $19950531 / 2300$ & 6.96 & 1.56 & 12.09 & 5.09 & 744 \\
\hline ST1 & SCAT & 28.75 & $19950501 / 0000$ & $19950531 / 2300$ & 7.01 & 1.64 & 12.22 & 5.08 & 744 \\
\hline ST1 & TPOD & 31.50 & $19950501 / 0000$ & $19950531 / 2300$ & 7.07 & 1.71 & 12.27 & 5.08 & 744 \\
\hline ST1 & VMCM & 37.50 & $19950501 / 0000$ & $19950531 / 2300$ & 7.26 & 1.87 & 12.40 & 5.08 & 744 \\
\hline ST1 & $\mathrm{VMCM}$ & 40.75 & $19950501 / 0000$ & $19950531 / 2300$ & 7.38 & 1.95 & 12.46 & 5.08 & 744 \\
\hline ST1 & TPOD & 46.00 & $19950501 / 0000$ & $19950531 / 2300$ & 7.59 & 2.06 & 12.45 & 5.05 & 744 \\
\hline ST1 & VMCM & 46.60 & $19950501 / 0000$ & $19950531 / 2300$ & 7.61 & 2.07 & 12.44 & 5.04 & 744 \\
\hline \multicolumn{10}{|c|}{ May 1995 Statistics Continued on Next Page } \\
\hline
\end{tabular}


Table 20: Monthly Hourly-Averaged Statistics

\begin{tabular}{|c|c|c|c|c|c|c|c|c|c|}
\hline Sta & $\begin{array}{l}\text { Inst } \\
\text { Type } \\
(\mathbf{m})\end{array}$ & $\begin{array}{l}\text { Sensor } \\
\text { Height } \\
\quad(\mathrm{m})\end{array}$ & $\begin{array}{c}\text { GMT } \\
\text { Start Time } \\
(\text { y m d/h m) }\end{array}$ & $\begin{array}{c}\text { GMT } \\
\text { Stop Time } \\
(\mathrm{y} \mathrm{m} \mathrm{d/h} \mathrm{m)}\end{array}$ & Mean & $\begin{array}{l}\text { Std } \\
\text { Dev }\end{array}$ & $\operatorname{Max}$ & Min & Npts \\
\hline \multicolumn{10}{|c|}{ May 1995 Water Temperature $\left({ }^{\circ} \mathrm{C}\right)$ (Continued) } \\
\hline $\mathrm{ST} 1$ & SCAT & 61.50 & $19950501 / 0000$ & $19950531 / 2300$ & 8.41 & 2.35 & 13.77 & 4.61 & 744 \\
\hline ST1 & TPOD & 64.50 & $19950501 / 0000$ & $19950531 / 2300$ & 8.58 & 2.34 & 13.71 & 5.00 & 744 \\
\hline ST1 & VMCM & 65.10 & $19950501 / 0000$ & $19950531 / 2300$ & 8.60 & 2.35 & 13.68 & 4.88 & 744 \\
\hline ST1 & SCAT & 67.50 & $19950501 / 0000$ & $19950531 / 2300$ & 8.76 & 2.32 & 13.85 & 5.12 & 744 \\
\hline $\mathrm{BS} 2$ & BASS & 70.98 & - & - & - & - & 一 & - & - \\
\hline BS2 & BASS & 72.57 & - & - & - & 一 & 一 & - & - \\
\hline BS2 & BASS & 73.52 & - & - & - & - & - & - & - \\
\hline BS2 & BASS & 74.47 & - & 一 & - & - & - & - & - \\
\hline $\mathrm{BS} 2$ & BASS & 75.18 & - & - & - & - & - & - & - \\
\hline $\mathrm{BS} 2$ & BASS & 75.82 & 一 & - & - & - & - & - & - \\
\hline BS2 & BASS & 76.42 & - & 一 & - & - & - & - & - \\
\hline $\mathrm{BS} 2$ & BASS & 76.68 & - & - & - & - & - & - & - \\
\hline ST2 & SCAT & 1.00 & $19950501 / 0000$ & $19950531 / 2300$ & 7.46 & 1.33 & 10.60 & 5.57 & 744 \\
\hline ST2 & TPOD & 5.40 & $19950501 / 0000$ & $19950531 / 2300$ & 7.23 & 1.23 & 9.96 & 5.38 & 744 \\
\hline ST2 & SCAT & 11.00 & $19950501 / 0000$ & $19950531 / 2300$ & 7.03 & 1.07 & 9.67 & 5.34 & 744 \\
\hline ST2 & VMCM & 17.00 & $19950501 / 0000$ & $19950517 / 1600$ & 6.22 & 0.52 & 9.94 & 5.31 & 401 \\
\hline ST2 & TPOD & 26.10 & $19950501 / 0000$ & $19950531 / 2300$ & 6.66 & 0.70 & 10.32 & 5.29 & 744 \\
\hline $\mathrm{ST} 2$ & SCAT & 32.00 & $19950501 / 0000$ & $19950531 / 2300$ & 6.74 & 0.78 & 10.56 & 5.37 & 744 \\
\hline ST2 & $\mathrm{VMCM}$ & 37.70 & $19950501 / 0000$ & $19950531 / 2300$ & 6.83 & 0.88 & 10.82 & 5.47 & 744 \\
\hline ST2 & TPOD & 42.80 & $19950501 / 0000$ & $19950531 / 2300$ & 6.86 & 0.94 & 11.04 & 5.46 & 744 \\
\hline ST2 & VACM & 61.50 & $19950501 / 0000$ & $19950531 / 2300$ & 7.03 & 1.07 & 11.13 & 5.45 & 744 \\
\hline $\mathrm{CR}$ & SBTC & 2.00 & $19950501 / 0000$ & $19950531 / 2300$ & 7.30 & 0.64 & 9.01 & 6.45 & 744 \\
\hline $\mathrm{CR}$ & SBTC & 10.00 & $19950501 / 0000$ & $19950531 / 2300$ & 7.28 & 0.64 & 8.99 & 6.42 & 744 \\
\hline SF & SBTC & 1.00 & $19950501 / 0000$ & $19950531 / 2300$ & 7.62 & 1.48 & 10.87 & 5.23 & 744 \\
\hline $\mathrm{SF}$ & SBTC & 5.00 & $19950501 / 0000$ & $19950531 / 2300$ & 7.40 & 1.35 & 10.46 & 5.23 & 744 \\
\hline SF & SBTC & 10.00 & $19950501 / 0000$ & $19950531 / 2300$ & 7.14 & 1.21 & 11.49 & 5.22 & 744 \\
\hline SF & SBTC & 15.00 & $19950501 / 0000$ & $19950531 / 2300$ & 7.00 & 1.15 & 12.57 & 5.22 & 744 \\
\hline SF & SBTC & 20.00 & $19950501 / 0000$ & $19950531 / 2300$ & 6.84 & 1.15 & 12.68 & 5.20 & 744 \\
\hline $\mathrm{SF}$ & SBTC & 25.00 & $19950501 / 0000$ & $19950531 / 2300$ & 6.81 & 1.27 & 12.98 & 5.20 & 744 \\
\hline $\mathrm{SF}$ & SBTC & 30.00 & $19950501 / 0000$ & $19950531 / 2300$ & 6.89 & 1.44 & 13.20 & 4.98 & 744 \\
\hline SF & SBTC & 35.00 & $19950501 / 0000$ & $19950531 / 2300$ & 7.01 & 1.59 & 13.12 & 4.96 & 744 \\
\hline $\mathrm{SF}$ & SBTC & 40.00 & - & - & 一 & - & - & - & - \\
\hline SF & SBTC & 45.00 & $19950501 / 0000$ & $19950531 / 2300$ & 7.38 & 1.88 & 12.97 & 5.00 & 744 \\
\hline \multirow[t]{2}{*}{$\mathrm{SF}$} & SBTC & 50.00 & $19950501 / 0000$ & $19950531 / 2300$ & 7.56 & 1.98 & 12.97 & 4.99 & 744 \\
\hline & \multicolumn{9}{|c|}{ May 1995 Statistics Continued on Next Page } \\
\hline
\end{tabular}


Table 20: Monthly Hourly-Averaged Statistics

\begin{tabular}{|c|c|c|c|c|c|c|c|c|c|}
\hline Sta & $\begin{array}{l}\text { Inst } \\
\text { Type } \\
(\mathrm{m})\end{array}$ & $\begin{array}{l}\text { Sensor } \\
\text { Height } \\
\text { (m) }\end{array}$ & $\begin{array}{c}\text { GMT } \\
\text { Start Time } \\
(\mathrm{y} \mathrm{m} \mathrm{d/h} \mathrm{m)}\end{array}$ & $\begin{array}{c}\text { GMT } \\
\text { Stop Time } \\
(\mathrm{y} \mathrm{m} \mathrm{d/h} \mathrm{m)}\end{array}$ & Mean & $\begin{array}{l}\text { Std } \\
\text { Dev }\end{array}$ & $\operatorname{Max}$ & Min & Npts \\
\hline \multicolumn{10}{|c|}{ May 1995 Water Temperature $\left({ }^{\circ} \mathrm{C}\right)$ (Continued) } \\
\hline $\mathrm{SF}$ & PMEL & 1.00 & $19950501 / 0000$ & $19950531 / 2300$ & 7.59 & 1.47 & 10.68 & 5.20 & 744 \\
\hline $\mathrm{SF}$ & PMEL & 10.00 & $19950501 / 0000$ & $19950531 / 2300$ & 7.18 & 1.22 & 11.64 & 5.24 & 744 \\
\hline $\mathrm{SF}$ & PMEL & 20.00 & $19950501 / 0000$ & $19950531 / 2300$ & 6.84 & 1.16 & 12.59 & 5.21 & 744 \\
\hline $\mathrm{SF}$ & PMEL & 50.00 & $19950501 / 0000$ & $19950531 / 2300$ & 7.24 & 1.80 & 12.98 & 4.97 & 744 \\
\hline NP & $\mathrm{RCM}$ & 23.00 & $19950501 / 0000$ & $19950531 / 2300$ & 5.93 & 0.29 & 6.65 & 5.28 & 744 \\
\hline NP & RCM & 37.00 & $19950501 / 0000$ & $19950531 / 2300$ & 5.92 & 0.29 & 6.59 & 5.28 & 744 \\
\hline NP & RCM & 59.00 & $19950501 / 0000$ & $19950531 / 2300$ & 5.93 & 0.28 & 6.57 & 5.30 & 744 \\
\hline NP & TG & 73.00 & - & - & - & - & - & - & - \\
\hline \multicolumn{10}{|c|}{ June 1995 Water Temperature $\left({ }^{\circ} \mathrm{C}\right)$} \\
\hline ST1 & IMET & 1.00 & $19950601 / 0000$ & $19950630 / 2300$ & 10.65 & 1.21 & 15.46 & 8.89 & 720 \\
\hline ST1 & VAWR & 1.50 & $19950601 / 0000$ & $19950630 / 2300$ & 10.57 & 1.11 & 14.36 & 8.89 & 720 \\
\hline ST1 & SCAT & 2.00 & $19950601 / 0000$ & $19950630 / 2300$ & 10.50 & 1.03 & 13.79 & 8.84 & 720 \\
\hline ST1 & TPOD & 3.50 & $19950601 / 0000$ & $19950630 / 2300$ & 10.34 & 0.88 & 13.25 & 8.76 & 720 \\
\hline ST1 & MTR & 5.65 & $19950601 / 0000$ & $19950630 / 2300$ & 10.10 & 0.73 & 12.79 & 8.22 & 720 \\
\hline ST1 & $\mathrm{VMCM}$ & 6.25 & $19950601 / 0000$ & $19950630 / 2300$ & 10.05 & 0.7 .2 & 12.50 & 8.11 & 720 \\
\hline ST1 & SCAT & 7.99 & $19950601 / 0000$ & $19950630 / 2300$ & 9.83 & 0.68 & 12.08 & 7.81 & 720 \\
\hline ST1 & $\mathrm{VMCM}$ & 10.45 & $19950601 / 0000$ & $19950630 / 2300$ & 9.56 & 0.69 & 11.71 & 7.49 & 720 \\
\hline ST1 & $\mathrm{VMCM}$ & 11.05 & $19950601 / 0000$ & $19950630 / 2300$ & 9.51 & 0.69 & 11.71 & 7.37 & 720 \\
\hline ST1 & SCAT & 12.75 & $19950601 / 0000$ & $19950630 / 2300$ & 9.30 & 0.72 & 11.41 & 7.09 & 720 \\
\hline ST1 & TPOD & 14.50 & $19950601 / 0000$ & $19950630 / 2300$ & 9.04 & 0.73 & 11.22 & 6.86 & 720 \\
\hline ST1 & $\mathrm{VMCM}$ & 19.50 & $19950601 / 0000$ & $19950630 / 2300$ & 8.32 & 0.75 & 10.90 & 6.36 & 720 \\
\hline ST1 & VMCM & 22.50 & $19950601 / 0000$ & $19950630 / 2300$ & 7.95 & 0.74 & 10.62 & 6.14 & 720 \\
\hline ST1 & TPOD & 25.50 & $19950601 / 0000$ & $19950630 / 2300$ & 7.63 & 0.67 & 10.26 & 6.14 & 720 \\
\hline ST1 & $\mathrm{VMCM}$ & 26.10 & $19950601 / 0000$ & $19950630 / 2300$ & 7.60 & 0.66 & 10.17 & 6.04 & 720 \\
\hline ST1 & SCAT & 28.75 & $19950601 / 0000$ & $19950630 / 2300$ & 7.41 & 0.63 & 9.63 & 5.89 & 720 \\
\hline ST1 & TPOD & 31.50 & $19950601 / 0000$ & $19950630 / 2300$ & 7.29 & 0.57 & 8.88 & 5.95 & 720 \\
\hline ST1 & VMCM & 37.50 & $19950601 / 0000$ & $19950630 / 2300$ & 7.15 & 0.53 & 8.77 & 6.30 & 720 \\
\hline ST1 & VMCM & 40.75 & $19950601 / 0000$ & $19950630 / 2300$ & 7.12 & 0.51 & 8.69 & 6.39 & 720 \\
\hline ST1 & TPOD & 46.00 & $19950601 / 0000$ & $19950630 / 2300$ & 7.08 & 0.48 & 8.53 & 6.44 & 720 \\
\hline ST1 & VMCM & 46.60 & $19950601 / 0000$ & $19950630 / 2300$ & 7.08 & 0.48 & 8.53 & 6.45 & 720 \\
\hline ST1 & SCAT & 61.50 & $19950601 / 0000$ & $19950630 / 2300$ & 7.00 & 0.40 & 8.18 & 6.45 & 720 \\
\hline ST1 & TPOD & 64.50 & $19950601 / 0000$ & $19950630 / 2300$ & 6.99 & 0.39 & 8.04 & 6.45 & 720 \\
\hline ST1 & $\mathrm{VMCM}$ & 65.10 & $19950601 / 0000$ & $19950630 / 2300$ & 6.99 & 0.39 & 8.06 & 6.45 & 720 \\
\hline ST1 & SCAT & 67.50 & $19950601 / 0000$ & $19950630 / 2300$ & 6.99 & 0.38 & 8.05 & 6.45 & 720 \\
\hline \multicolumn{10}{|c|}{ June 1995 Statistics Continued on Next Page } \\
\hline
\end{tabular}


Table 20: Monthly Hourly-Averaged Statistics

\begin{tabular}{|c|c|c|c|c|c|c|c|c|c|}
\hline Sta & $\begin{array}{l}\text { Inst } \\
\text { Type } \\
(\mathbf{m})\end{array}$ & $\begin{array}{l}\text { Sensor } \\
\text { Height } \\
\text { (m) }\end{array}$ & $\begin{array}{c}\text { GMT } \\
\text { Start Time } \\
(\mathrm{y} \mathrm{m} \mathrm{d/h} \mathrm{m)}\end{array}$ & $\begin{array}{c}\text { GMT } \\
\text { Stop Time } \\
(\mathbf{y ~ m ~ d / h ~ m ) ~}\end{array}$ & Mean & $\begin{array}{l}\text { Std } \\
\text { Dev }\end{array}$ & Max & Min & Npts \\
\hline \multicolumn{10}{|c|}{ June 1995 Water Temperature $\left({ }^{\circ} \mathrm{C}\right)$ (Continued) } \\
\hline $\mathrm{BS} 2$ & BASS & 70.98 & - & - & - & - & - & - & - \\
\hline $\mathrm{BS} 2$ & BASS & 72.57 & - & - & - & - & - & - & - \\
\hline $\mathrm{BS} 2$ & BASS & 73.52 & - & - & - & - & - & - & 一 \\
\hline $\mathrm{BS} 2$ & BASS & 74.47 & - & - & - & - & - & - & - \\
\hline $\mathrm{BS} 2$ & BASS & 75.18 & - & - & - & - & - & - & - \\
\hline BS2 & BASS & 75.82 & - & 一 & - & - & - & - & - \\
\hline $\mathrm{BS} 2$ & BASS & 76.42 & - & - & - & - & - & - & - \\
\hline $\mathrm{BS} 2$ & BASS & 76.68 & - & - & - & - & - & - & - \\
\hline ST2 & SCAT & 1.00 & $19950601 / 0000$ & $19950630 / 2300$ & 10.35 & 1.30 & 14.75 & 8.35 & 720 \\
\hline ST2 & TPOD & 5.40 & $19950601 / 0000$ & $19950630 / 2300$ & 9.71 & 0.79 & 13.26 & 7.99 & 720 \\
\hline ST2 & SCAT & 11.00 & $19950601 / 0000$ & $19950630 / 2300$ & 9.14 & 0.76 & 11.49 & 7.42 & 720 \\
\hline ST2 & VMCM & 17.00 & - & - & - & - & - & - & - \\
\hline ST2 & TPOD & 26.10 & $19950601 / 0000$ & $19950630 / 2300$ & 7.89 & 0.77 & 10.27 & 6.65 & 720 \\
\hline ST2 & SCAT & 32.00 & $19950601 / 0000$ & $19950630 / 2300$ & 7.78 & 0.70 & 10.02 & 6.66 & 720 \\
\hline ST2 & VMCM & 37.70 & $19950601 / 0000$ & $19950630 / 2300$ & 7.72 & 0.67 & 9.42 & 6.67 & 720 \\
\hline ST2 & TPOD & 42.80 & $19950601 / 0000$ & $19950630 / 2300$ & 7.66 & 0.65 & 9.39 & 6.65 & 720 \\
\hline ST2 & VACM & 61.50 & $19950601 / 0000$ & $19950630 / 2300$ & 7.62 & 0.61 & 9.11 & 6.66 & 720 \\
\hline $\mathrm{CR}$ & SBTC & 2.00 & $19950601 / 0000$ & $19950630 / 2300$ & 10.55 & 1.02 & 12.48 & 8.60 & 720 \\
\hline $\mathrm{CR}$ & SBTC & 10.00 & $19950601 / 0000$ & $19950630 / 2300$ & 10.52 & 1.01 & 12.31 & 8.59 & 720 \\
\hline SF & SBTC & 1.00 & $19950601 / 0000$ & $19950630 / 2300$ & 10.48 & 1.49 & 17.11 & 8.43 & 720 \\
\hline $\mathrm{SF}$ & SBTC & 5.00 & $19950601 / 0000$ & $19950630 / 2300$ & 9.91 & 0.94 & 12.71 & 8.36 & 720 \\
\hline $\mathrm{SF}$ & SBTC & 10.00 & $19950601 / 0000$ & $19950630 / 2300$ & 9.37 & 0.84 & 12.34 & 7.49 & 720 \\
\hline $\mathrm{SF}$ & SBTC & 15.00 & $19950601 / 0000$ & $19950630 / 2300$ & 8.64 & 0.78 & 11.62 & 6.71 & 720 \\
\hline $\mathrm{SF}$ & SBTC & 20.00 & $19950601 / 0000$ & $19950630 / 2300$ & 8.03 & 0.70 & 10.64 & 6.51 & 720 \\
\hline $\mathrm{SF}$ & SBTC & 25.00 & $19950601 / 0000$ & $19950630 / 2300$ & 7.61 & 0.62 & 10.06 & 6.43 & 720 \\
\hline $\mathrm{SF}$ & SBTC & 30.00 & $19950601 / 0000$ & $19950630 / 2300$ & 7.33 & 0.57 & 9.14 & 6.39 & 720 \\
\hline $\mathrm{SF}$ & SBTC & 35.00 & $19950601 / 0000$ & $19950630 / 2300$ & 7.20 & 0.53 & 8.59 & 6.38 & 720 \\
\hline $\mathrm{SF}$ & SBTC & 40.00 & - & - & - & - & - & - & - \\
\hline SF & SBTC & 45.00 & $19950601 / 0000$ & $19950630 / 2300$ & 7.11 & 0.49 & 8.56 & 6.39 & 720 \\
\hline $\mathrm{SF}$ & SBTC & 50.00 & $19950601 / 0000$ & $19950630 / 2300$ & 7.08 & 0.48 & 8.53 & 6.40 & 720 \\
\hline $\mathrm{SF}$ & PMEL & 1.00 & $19950601 / 0000$ & $19950630 / 2300$ & 10.43 & 1.42 & 15.97 & 8.42 & 720 \\
\hline $\mathrm{SF}$ & PMEL & 10.00 & $19950601 / 0000$ & $19950630 / 2300$ & 9.47 & 0.85 & 12.39 & 7.65 & 720 \\
\hline $\mathrm{SF}$ & PMEL & 20.00 & $19950601 / 0000$ & $19950630 / 2300$ & 7.97 & 0.69 & 10.53 & 6.49 & 720 \\
\hline \multirow[t]{2}{*}{$\mathrm{SF}$} & PMEL & 50.00 & $19950601 / 0000$ & $19950630 / 2300$ & 7.10 & 0.50 & 8.54 & 6.36 & 720 \\
\hline & \multicolumn{9}{|c|}{ June 1995 Statistics Continued on Next Page } \\
\hline
\end{tabular}


Table 20: Monthly Hourly-Averaged Statistics

\begin{tabular}{|c|c|c|c|c|c|c|c|c|c|}
\hline Sta & $\begin{array}{l}\text { Inst } \\
\text { Type } \\
(\mathbf{m})\end{array}$ & $\begin{array}{c}\text { Sensor } \\
\text { Height } \\
\text { (m) }\end{array}$ & $\begin{array}{c}\text { GMT } \\
\text { Start Time } \\
(\text { y } \mathrm{m} \mathrm{d} / \mathrm{h} \mathrm{m})\end{array}$ & $\begin{array}{c}\text { GMT } \\
\text { Stop Time } \\
(\mathbf{y ~} \mathbf{m ~ d} / \mathbf{h ~ m})\end{array}$ & Mean & $\begin{array}{l}\text { Std } \\
\text { Dev }\end{array}$ & $\operatorname{Max}$ & Min & Npts \\
\hline \multicolumn{10}{|c|}{ June 1995 Water Temperature $\left({ }^{\circ} \mathrm{C}\right)$ (Continued) } \\
\hline NP & $\mathrm{RCM}$ & 23.00 & $19950601 / 0000$ & $19950611 / 2300$ & 7.00 & 0.23 & 7.45 & 6.50 & 255 \\
\hline NP & RCM & 37.00 & $19950601 / 0000$ & $19950611 / 2300$ & & 0.22 & 7.41 & 6.48 & 255 \\
\hline NP & $\mathrm{RCM}$ & 59.00 & $19950601 / 0000$ & $19950611 / 2300$ & 6.98 & 0.22 & 7.43 & 6.48 & 255 \\
\hline NP & TG & 73.00 & - & - & 一 & - & - & - & - \\
\hline \multicolumn{10}{|c|}{ July 1995 Water Temperature $\left({ }^{\circ} \mathrm{C}\right)$} \\
\hline $\mathrm{ST} 1$ & IMET & 1.00 & $19950701 / 0000$ & $19950731 / 2300$ & 16.22 & 1.15 & 19.33 & 12.02 & 744 \\
\hline ST1 & VAWR & 1.50 & $19950701 / 0000$ & $19950731 / 2300$ & 16.09 & 1.15 & 18.92 & 11.74 & 744 \\
\hline ST1 & SCAT & 2.00 & $19950701 / 0000$ & $19950731 / 2300$ & 15.90 & 1.18 & 18.79 & 11.22 & 744 \\
\hline ST1 & TPOD & 3.50 & $19950701 / 0000$ & $19950731 / 2300$ & 15.44 & 1.31 & 18.45 & 10.79 & 744 \\
\hline ST1 & MTR & 5.65 & 1995 0701/0000 & $19950731 / 2300$ & 14.67 & 1.43 & 17.71 & 10.49 & 744 \\
\hline ST1 & $\mathrm{VMCM}$ & 6.25 & $19950701 / 0000$ & $19950731 / 2300$ & 14.52 & 1.44 & 17.67 & 10.46 & 744 \\
\hline ST1 & SCAT & 7.99 & $19950701 / 0000$ & $19950731 / 2300$ & 13.75 & 1.47 & 17.18 & 10.26 & 744 \\
\hline ST1 & $\mathrm{VMCM}$ & 10.45 & $19950701 / 0000$ & $19950731 / 2300$ & 12.86 & 1.40 & 16.42 & 8.74 & 744 \\
\hline ST1 & VMCM & 11.05 & $19950701 / 0000$ & $19950731 / 2300$ & 12.72 & 1.37 & 16.37 & 8.75 & 744 \\
\hline ST1 & SCAT & 12.75 & $19950701 / 0000$ & $19950731 / 2300$ & 12.15 & 1.36 & 16.62 & 8.53 & 744 \\
\hline ST1 & TPOD & 14.50 & $19950701 / 0000$ & $19950731 / 2300$ & 11.60 & 1.30 & 16.08 & 8.51 & 744 \\
\hline ST1 & $\mathrm{VMCM}$ & 19.50 & $19950701 / 0000$ & $19950731 / 2300$ & 10.42 & 1.16 & 14.44 & 7.95 & 744 \\
\hline ST1 & VMCM & 22.50 & $19950701 / 0000$ & $19950731 / 2300$ & 9.94 & 1.08 & 13.92 & 7.78 & 744 \\
\hline ST1 & TPOD & 25.50 & $19950701 / 0000$ & $19950731 / 2300$ & 9.52 & 0.95 & 12.72 & 7.62 & 744 \\
\hline ST1 & $\mathrm{VMCM}$ & 26.10 & $19950701 / 0000$ & $19950731 / 2300$ & 9.47 & 0.93 & 12.65 & 7.55 & 744 \\
\hline ST1 & SCAT & 28.75 & $19950701 / 0000$ & $19950731 / 2300$ & 9.22 & 0.91 & 12.65 & 7.21 & 744 \\
\hline ST1 & TPOD & 31.50 & $19950701 / 0000$ & $19950731 / 2300$ & 9.02 & 0.83 & 11.21 & 7.29 & 744 \\
\hline ST1 & VMCM & 37.50 & $19950701 / 0000$ & $19950731 / 2300$ & 8.76 & 0.77 & 10.72 & 7.27 & 744 \\
\hline ST1 & VMCM & 40.75 & $19950701 / 0000$ & $19950731 / 2300$ & 8.67 & 0.76 & 10.59 & 7.24 & 744 \\
\hline ST1 & TPOD & 46.00 & $19950701 / 0000$ & $19950731 / 2300$ & 8.56 & 0.72 & 10.38 & 7.21 & 744 \\
\hline ST1 & VMCM & 46.60 & $19950701 / 0000$ & $19950731 / 2300$ & 8.56 & 0.72 & 10.39 & 7.21 & 744 \\
\hline ST1 & SCAT & 61.50 & $19950701 / 0000$ & $19950731 / 2300$ & 8.31 & 0.60 & 9.62 & 7.13 & 744 \\
\hline ST1 & TPOD & 64.50 & $19950701 / 0000$ & $19950731 / 2300$ & 8.27 & 0.58 & 9.59 & 7.13 & 744 \\
\hline ST1 & $\mathrm{VMCM}$ & 65.10 & $19950701 / 0000$ & $19950731 / 2300$ & 8.27 & 0.58 & 9.59 & 7.13 & 744 \\
\hline ST1 & SCAT & 67.50 & $19950701 / 0000$ & $19950731 / 2300$ & 8.25 & 0.57 & 9.57 & 7.13 & 744 \\
\hline $\mathrm{BS} 2$ & BASS & 70.98 & $19950711 / 1900$ & $19950731 / 1300$ & 8.69 & 0.39 & 9.69 & 7.89 & 486 \\
\hline $\mathrm{BS} 2$ & BASS & 72.57 & $19950711 / 1900$ & $19950731 / 1300$ & 8.68 & 0.38 & 9.71 & 7.60 & 486 \\
\hline BS2 & BASS & 73.52 & $19950711 / 1900$ & $19950731 / 1300$ & 8.69 & 0.38 & 9.69 & 7.90 & 486 \\
\hline $\mathrm{BS} 2$ & BASS & 74.47 & $19950711 / 1900$ & $19950731 / 1300$ & 8.68. & 0.38 & 9.69 & 7.88 & 486 \\
\hline BS2 & BASS & 75.18 & 1995 0711/1900 & 1995 0731/1300 & 8.72 & 0.36 & 9.71 & 7.93 & 486 \\
\hline $\mathrm{BS} 2$ & BASS & 75.82 & $19950711 / 1900$ & $19950731 / 1300$ & 8.68 & 0.38 & 9.69 & 7.89 & 486 \\
\hline BS2 & BASS & 76.42 & 1995 0711/1900 & $19950731 / 1300$ & 8.68 & 0.38 & 9.68 & 7.88 & 486 \\
\hline BS2 & BASS & 76.68 & $19950711 / 1900$ & $19950731 / 1300$ & 8.68 & 0.38 & 9.69 & 7.88 & 486 \\
\hline \multicolumn{10}{|c|}{ July 1995 Statistics Continued on Next Page } \\
\hline
\end{tabular}


Table 20: Monthly Hourly-Averaged Statistics

\begin{tabular}{|c|c|c|c|c|c|c|c|c|c|}
\hline Sta & $\begin{array}{l}\text { Inst } \\
\text { Type } \\
(\mathrm{m})\end{array}$ & $\begin{array}{l}\text { Sensor } \\
\text { Height } \\
\text { (m) }\end{array}$ & $\begin{array}{c}\text { GMT } \\
\text { Start Time } \\
(\mathbf{y} \mathrm{m} \mathrm{d} / \mathbf{h} \mathbf{m})\end{array}$ & $\begin{array}{c}\text { GMT } \\
\text { Stop Time } \\
(\mathbf{y ~} \mathbf{m} \text { d/h m) }\end{array}$ & Mean & $\begin{array}{l}\text { Std } \\
\text { Dev }\end{array}$ & $\operatorname{Max}$ & Min & Npts \\
\hline \multicolumn{10}{|c|}{ July 1995 Water Temperature $\left({ }^{\circ} \mathrm{C}\right)$ (Continued) } \\
\hline ST2 & SCAT & 1.00 & $19950701 / 0000$ & $19950731 / 2300$ & 15.43 & 1.41 & 18.39 & 10.89 & 744 \\
\hline ST2 & TPOD & 5.40 & $19950701 / 0000$ & $19950731 / 2300$ & 13.36 & 1.52 & 16.82 & 10.36 & 744 \\
\hline $\mathrm{ST} 2$ & SCAT & 11.00 & $19950701 / 0000$ & $19950731 / 2300$ & 11.58 & 1.15 & 15.09 & 9.60 & 744 \\
\hline ST2 & $\mathrm{VMCM}$ & 17.00 & - & - & - & - & - & - & - \\
\hline ST2 & TPOD & 26.10 & $19950701 / 0000$ & $19950731 / 2300$ & 9.86 & 0.84 & 14.74 & 7.80 & 744 \\
\hline ST2 & SCAT & 32.00 & $19950701 / 0000$ & $19950731 / 2300$ & 9.71 & 0.79 & 11.73 & 7.78 & 744 \\
\hline ST2 & $\mathrm{VMCM}$ & 37.70 & $19950701 / 0000$ & $19950727 / 1000$ & 9.35 & 0.59 & 10.95 & 7.77 & 635 \\
\hline ST2 & TPOD & 42.80 & $19950701 / 0000$ & $19950731 / 2300$ & 9.42 & 0.74 & 11.47 & 7.74 & 744 \\
\hline $\mathrm{ST} 2$ & VACM & 61.50 & $19950701 / 0000$ & $19950731 / 2300$ & 9.23 & 0.63 & 11.05 & 7.75 & 744 \\
\hline CR & SBTC & 2.00 & $19950701 / 0000$ & $19950731 / 2300$ & 13.75 & 0.89 & 15.55 & 12.10 & 744 \\
\hline CR & SBTC & 10.00 & $19950701 / 0000$ & $19950731 / 2300$ & 13.72 & 0.88 & 15.50 & 12.06 & 744 \\
\hline SF & SBTC & 1.00 & $19950701 / 0000$ & $19950731 / 2300$ & & 1.49 & & 11.77 & 744 \\
\hline $\mathrm{SF}$ & SBTC & 5.00 & $19950701 / 0000$ & $19950731 / 2300$ & 14.14 & 1.50 & 17.00 & 10.63 & 744 \\
\hline $\mathrm{SF}$ & SBTC & 10.00 & $19950701 / 0000$ & $19950731 / 2300$ & 12.49 & 1.29 & & 9.67 & 744 \\
\hline SF & SBTC & 15.00 & $19950701 / 0000$ & $19950731 / 2300$ & 11.14 & 1.11 & 14.51 & 8.71 & 744 \\
\hline $\mathrm{SF}$ & SBTC & 20.00 & $19950701 / 0000$ & $19950731 / 2300$ & 10.13 & 1.11 & 14.88 & 8.28 & 744 \\
\hline $\mathrm{SF}$ & SBTC & 25.00 & $19950701 / 0000$ & $19950731 / 2300$ & 9.52 & 0.98 & 13.48 & 7.79 & 744 \\
\hline $\mathrm{SF}$ & SBTC & 30.00 & $19950701 / 0000$ & $0731 / 2300$ & 9.14 & 0.83 & 12.36 & 7.56 & 744 \\
\hline $\mathrm{SF}$ & SBTC & 35.00 & $19950701 / 0000$ & $19950731 / 2300$ & 8.93 & 0.77 & 11.09 & 7.46 & 744 \\
\hline $\mathrm{SF}$ & SBTC & 40.00 & - & - & - & - & - & - & - \\
\hline SF & SBTC & 45.00 & $19950701 / 0000$ & $19950731 / 2300$ & 8.70 & 0.68 & & 7.44 & 744 \\
\hline $\mathrm{SF}$ & SBTC & 50.00 & $19950701 / 0000$ & $19950731 / 2300$ & 8.61 & 0.63 & 10.40 & 7.43 & 744 \\
\hline $\mathrm{SF}$ & PMEL & 1.00 & $19950701 / 0000$ & $19950731 / 2300$ & & & & 11.80 & 744 \\
\hline $\mathrm{SF}$ & PMEL & 10.00 & $19950701 / 0000$ & $19950731 / 2300$ & 12.71 & 1.33 & 16.27 & 9.90 & 744 \\
\hline $\mathrm{SF}$ & PMEL & 20.00 & $19950701 / 0000$ & $19950731 / 2300$ & 10.05 & 1.10 & 14.58 & 7.94 & 744 \\
\hline $\mathrm{SF}$ & PMEL & 50.00 & $19950701 / 0000$ & $19950731 / 2300$ & 8.74 & 0.71 & 10.95 & 7.42 & 744 \\
\hline NP & RCM & 23.00 & - & - & - & - & 一 & - & - \\
\hline NP & $\mathrm{RCM}$ & 37.00 & - & - & - & - & - & - & - \\
\hline NP & $\mathrm{RCM}$ & 59.00 & - & - & - & - & - & - & 一 \\
\hline NP & TG & 73.00 & - & - & - & - & - & - & - \\
\hline \multicolumn{10}{|c|}{ August 1995 Water Temperature $\left({ }^{\circ} \mathrm{C}\right)$} \\
\hline ST1 & IMET & 1.00 & $19950801 / 0000$ & $19950823 / 1600$ & 20.34 & 2.99 & 25.53 & 14.89 & 545 \\
\hline ST1 & VAWR & 1.50 & $19950801 / 0000$ & $19950823 / 1600$ & 20.31 & 3.01 & 25.51 & 14.87 & 545 \\
\hline ST1 & SCAT & 2.00 & $19950801 / 0000$ & $19950823 / 1500$ & 20.26 & 3.04 & 25.47 & 14.92 & 544 \\
\hline ST1 & TPOD & 3.50 & $19950801 / 0000$ & $19950823 / 1600$ & 20.19 & 3.12 & 25.29 & 14.79 & 545 \\
\hline \multicolumn{10}{|c|}{ August 1995 Statistics Continued on Next Page } \\
\hline
\end{tabular}


Table 20: Monthly Hourly-Averaged Statistics

\begin{tabular}{|c|c|c|c|c|c|c|c|c|c|}
\hline Sta & $\begin{array}{l}\text { Inst } \\
\text { Type } \\
(\mathbf{m})\end{array}$ & $\begin{array}{l}\text { Sensor } \\
\text { Height } \\
\quad(\mathrm{m})\end{array}$ & $\begin{array}{c}\text { GMT } \\
\text { Start Time } \\
(\mathbf{y} \mathbf{m ~ d} / \mathbf{h ~ m})\end{array}$ & $\begin{array}{c}\text { GMT } \\
\text { Stop Time } \\
(\mathbf{y} \mathbf{m ~ d} / \mathbf{h ~ m})\end{array}$ & Mean & $\begin{array}{l}\text { Std } \\
\text { Dev }\end{array}$ & Max & Min & Npts \\
\hline \multicolumn{10}{|c|}{ August 1995 Water Temperature $\left({ }^{\circ} \mathrm{C}\right)$ (Continued) } \\
\hline ST1 & MTR & 5.65 & $19950801 / 0000$ & $19950823 / 1600$ & 20.07 & 3.34 & 24.99 & 14.46 & 545 \\
\hline ST1 & VMCM & 6.25 & $19950801 / 0000$ & $19950823 / 1600$ & 20.04 & 3.38 & 24.97 & 14.25 & 545 \\
\hline ST1 & SCAT & 7.99 & $19950801 / 0000$ & $19950823 / 1600$ & 19.89 & 3.63 & 24.98 & 13.30 & 545 \\
\hline ST1 & VMCM & 10.45 & $19950801 / 0000$ & $19950823 / 1600$ & 19.56 & 4.00 & 24.97 & 11.54 & 545 \\
\hline ST1 & VMCM & 11.05 & $19950801 / 0000$ & $19950823 / 1600$ & 19.57 & 4.09 & 24.96 & 11.74 & 545 \\
\hline ST1 & SCAT & 12.75 & $19950801 / 0000$ & $19950823 / 1600$ & 19.32 & 4.34 & 24.96 & 10.97 & 545 \\
\hline ST1 & TPOD & 14.50 & $19950801 / 0000$ & $19950823 / 1600$ & 18.98 & 4.57 & 24.92 & 10.76 & 545 \\
\hline ST1 & $\mathrm{VMCM}$ & 19.50 & $19950801 / 0000$ & $19950823 / 1600$ & 17.87 & 5.07 & 24.85 & 10.05 & 545 \\
\hline ST1 & VMCM & 22.50 & $19950801 / 0000$ & $19950823 / 1600$ & 17.19 & 5.28 & 24.82 & 9.57 & 545 \\
\hline ST1 & TPOD & 25.50 & $19950801 / 0000$ & $19950823 / 1600$ & 16.31 & 5.30 & 24.75 & 9.45 & 545 \\
\hline ST1 & $\mathrm{VMCM}$ & 26.10 & $19950801 / 0000$ & $19950823 / 1600$ & 16.19 & 5.29 & 24.74 & 9.44 & 545 \\
\hline ST1 & SCAT & 28.75 & $19950801 / 0000$ & $19950823 / 1600$ & 15.41 & 5.23 & 24.64 & 9.11 & 545 \\
\hline ST1 & TPOD & 31.50 & $19950801 / 0000$ & $19950823 / 1600$ & 14.59 & 4.98 & 24.52 & 8.75 & 545 \\
\hline ST1 & VMCM & 37.50 & $19950801 / 0000$ & $19950823 / 1600$ & 12.97 & 4.16 & 24.44 & 8.58 & 545 \\
\hline ST1 & VMCM & 40.75 & $19950801 / 0000$ & $19950823 / 1600$ & 12.27 & 3.66 & 24.41 & 8.50 & 545 \\
\hline ST1 & TPOD & 46.00 & $19950801 / 0000$ & $19950823 / 1600$ & 11.30 & 2.64 & 23.49 & 8.43 & 545 \\
\hline ST1 & VMCM & 46.60 & $19950801 / 0000$ & $19950817 / 0400$ & 10.09 & 1.41 & 18.82 & 8.43 & 389 \\
\hline ST1 & SCAT & 61.50 & $19950801 / 0000$ & $19950823 / 1600$ & 10.24 & 1.46 & 12.76 & 8.34 & 545 \\
\hline ST1 & TPOD & 64.50 & $19950801 / 0000$ & $19950823 / 1600$ & 10.22 & 1.46 & 12.57 & 8.34 & 545 \\
\hline ST1 & $\mathrm{VMCM}$ & 65.10 & $19950801 / 0000$ & $19950823 / 1600$ & 10.22 & 1.46 & 12.57 & 8.34 & 545 \\
\hline ST1 & SCAT & 67.50 & $19950801 / 0000$ & $19950823 / 1600$ & 10.21 & 1.47 & 12.52 & 8.34 & 545 \\
\hline BS2 & BASS & & & $19950824 / 13$ & & 1.5 & & 8.46 & 533 \\
\hline BS2 & BASS & 72.57 & $19950801 / 0018$ & $19950824 / 1318$ & 10.27 & 1.50 & 12.68 & 8.46 & 507 \\
\hline BS2 & BASS & 73.52 & $19950801 / 0018$ & $19950824 / 1318$ & 10.33 & 1.50 & 12.65 & 8.46 & 533 \\
\hline BS2 & BASS & 74.47 & 1995 0801/0018 & $19950824 / 1318$ & 10.33 & 1.50 & 12.66 & 8.46 & 533 \\
\hline $\mathrm{BS} 2$ & BASS & 75.18 & $19950801 / 0018$ & $19950824 / 1318$ & 10.33 & 1.50 & 12.66 & 8.46 & 533 \\
\hline $\mathrm{BS} 2$ & BASS & 75.82 & $19950801 / 0018$ & $19950824 / 1318$ & 10.34 & 1.50 & 12.66 & 8.46 & 533 \\
\hline $\mathrm{BS} 2$ & BASS & 76.42 & $19950801 / 0018$ & $19950824 / 1318$ & 10.33 & 1.50 & 12.66 & 8.46 & 533 \\
\hline $\mathrm{BS} 2$ & BASS & 76.68 & $19950801 / 0018$ & $19950824 / 1318$ & 10.34 & 1.51 & 12.67 & 8.46 & 533 \\
\hline $\mathrm{ST} 2$ & SCAT & & & $19950804 / 2300$ & & & 18.65 & 16.50 & 96 \\
\hline $\mathrm{ST} 2$ & TPOD & 5.40 & $19950801 / 0000$ & $19950804 / 2300$ & 16.43 & 0.85 & 17.97 & 13.87 & 96 \\
\hline ST2 & SCAT & 11.00 & $19950801 / 0000$ & $19950804 / 2300$ & 14.62 & 0.88 & 16.91 & 12.77 & 96 \\
\hline ST2 & $\mathrm{VMCM}$ & 17.00 & - & - & - & - & - & - & - \\
\hline ST2 & TPOD & 26.10 & $19950801 / 0000$ & $19950804 / 2300$ & 10.50 & 0.63 & 12.46 & 9.26 & 96 \\
\hline ST2 & SCAT & 32.00 & $19950801 / 0000$ & $19950804 / 2300$ & 9.95 & 0.44 & 11.39 & 8.98 & 96 \\
\hline ST2 & VMCM & 37.70 & - & - & - & - & - & - & - \\
\hline ST2 & TPOD & 42.80 & $19950801 / 0000$ & $19950804 / 2300$ & 9.13 & 0.44 & 10.18 & 8.09 & 96 \\
\hline \multirow[t]{2}{*}{$\mathrm{ST} 2$} & VACM & 61.50 & $19950801 / 0000$ & $19950804 / 2300$ & 9.58 & 0.50 & 10.65 & 8.75 & 96 \\
\hline & \multicolumn{9}{|c|}{ August 1995 Statistics Continued on Next Page } \\
\hline
\end{tabular}


Table 20: Monthly Hourly-Averaged Statistics

\begin{tabular}{|c|c|c|c|c|c|c|c|c|c|}
\hline Sta & $\begin{array}{l}\text { Inst } \\
\text { Type } \\
(\mathbf{m})\end{array}$ & $\begin{array}{l}\text { Sensor } \\
\text { Height } \\
\quad(\mathrm{m})\end{array}$ & $\begin{array}{c}\text { GMT } \\
\text { Start Time } \\
(\mathbf{y} \mathrm{m} \mathrm{d} / \mathrm{h} \mathrm{m})\end{array}$ & $\begin{array}{c}\text { GMT } \\
\text { Stop Time } \\
(\mathbf{y ~} \mathbf{m ~ d} / \mathbf{h ~ m})\end{array}$ & Mean & $\begin{array}{l}\text { Std } \\
\text { Dev }\end{array}$ & Max & Min & Npts \\
\hline \multicolumn{10}{|c|}{ August 1995 Water Temperature $\left({ }^{\circ} \mathrm{C}\right)$ (Continued) } \\
\hline $\mathrm{CR}$ & SBTC & 2.00 & $19950801 / 0000$ & $19950823 / 1600$ & 16.12 & 0.56 & 17.28 & 15.03 & 545 \\
\hline $\mathrm{CR}$ & SBTC & 10.00 & $19950801 / 0000$ & $19950823 / 1600$ & 16.09 & 0.56 & 17.10 & 15.02 & 545 \\
\hline SF & SBTC & 1.00 & $19950801 / 0000$ & $19950823 / 1600$ & 18.68 & 2.26 & 25.82 & 14.25 & 545 \\
\hline $\mathrm{SF}$ & SBTC & 5.00 & $19950801 / 0000$ & $19950823 / 1600$ & 18.19 & 2.68 & 24.32 & 12.52 & 545 \\
\hline $\mathrm{SF}$ & SBTC & 10.00 & $19950801 / 0000$ & $19950823 / 1600$ & 17.61 & 3.56 & 24.17 & 11.50 & 545 \\
\hline $\mathrm{SF}$ & SBTC & 15.00 & $19950801 / 0000$ & $19950823 / 1600$ & 16.89 & 4.00 & 24.20 & 10.54 & 545 \\
\hline SF & SBTC & 20.00 & $19950801 / 0000$ & $19950823 / 1600$ & 15.64 & 3.86 & 23.48 & 10.24 & 545 \\
\hline $\mathrm{SF}$ & SBTC & 25.00 & $19950801 / 0000$ & $19950823 / 1600$ & 14.24 & 3.48 & 22.91 & 9.86 & 545 \\
\hline $\mathrm{SF}$ & SBTC & 30.00 & $19950801 / 0000$ & $19950823 / 1600$ & 12.95 & 2.93 & 22.74 & 9.38 & 545 \\
\hline SF & SBTC & 35.00 & $19950801 / 0000$ & $19950823 / 1600$ & 11.98 & 2.24 & 21.83 & 8.89 & 545 \\
\hline $\mathrm{SF}$ & SBTC & 40.00 & - & - & - & - & - & - & - \\
\hline $\mathrm{SF}$ & SBTC & 45.00 & $19950801 / 0000$ & 1995 0823/1600 & 10.88 & 1.30 & 15.31 & 8.48 & 545 \\
\hline SF & SBTC & 50.00 & $19950801 / 0000$ & $19950823 / 1600$ & 10.64 & 1.24 & 13.45 & 8.48 & 545 \\
\hline SF & PMEL & 1.00 & $19950801 / 0000$ & $19950823 / 1600$ & 18.66 & 2.29 & 25.76 & 14.22 & 545 \\
\hline SF & PMEL & 10.00 & $19950801 / 0000$ & $19950823 / 1600$ & 17.76 & 3.50 & 24.23 & 11.55 & 545 \\
\hline SF & PMEL & 20.00 & $19950801 / 0000$ & $19950823 / 1600$ & 15.47 & 3.83 & 23.49 & 10.26 & 545 \\
\hline $\mathrm{SF}$ & PMEL & 50.00 & $19950801 / 0000$ & $19950823 / 1600$ & 11.16 & 1.56 & 20.23 & 8.49 & 545 \\
\hline NP & $\mathrm{RCM}$ & 23.00 & - & - & - & - & - & 一 & $\ldots$ \\
\hline NP & RCM & 37.00 & - & - & - & - & - & - & - \\
\hline NP & $\mathrm{RCM}$ & 59.00 & - & - & - & 一 & - & 一 & - \\
\hline NP & TG & 73.00 & - & - & - & - & - & - & - \\
\hline
\end{tabular}


Table 21: Monthly Salinity Hourly-Averaged Statistics

\begin{tabular}{|c|c|c|c|c|c|c|c|c|c|}
\hline Sta & $\begin{array}{l}\text { Inst } \\
\text { Type } \\
(\mathbf{m})\end{array}$ & $\begin{array}{l}\text { Sensor } \\
\text { Height } \\
\quad(\mathrm{m})\end{array}$ & $\begin{array}{c}\text { GMT } \\
\text { Start Time } \\
(\mathbf{y ~} \mathbf{m ~ d} / \mathbf{h ~ m})\end{array}$ & $\begin{array}{c}\text { GMT } \\
\text { Stop Time } \\
(\mathrm{y} \mathrm{m} \mathrm{d/h} \mathrm{m)}\end{array}$ & Mean & $\begin{array}{l}\text { Std } \\
\text { Dev }\end{array}$ & $\operatorname{Max}$ & Min & Npts \\
\hline \multicolumn{10}{|c|}{ February 1995 Salinity (PSU) } \\
\hline ST1 & IMET & 1.00 & $19950201 / 0000$ & $19950228 / 2300$ & 33.14 & 0.05 & 33.23 & 32.98 & 672 \\
\hline ST1 & VAWR & 1.50 & 1995 0201/0000 & $19950228 / 2300$ & 33.14 & 0.05 & 33.22 & 32.98 & 672 \\
\hline ST1 & SCAT & 2.00 & $19950201 / 0000$ & $19950228 / 2300$ & 33.15 & 0.05 & 33.22 & 32.98 & 672 \\
\hline ST1 & TPOD & 3.50 & 1995 0201/0000 & $19950228 / 2300$ & 33.15 & 0.05 & 33.22 & 33.00 & 672 \\
\hline ST1 & MTR & 5.65 & $19950201 / 0000$ & $19950228 / 2300$ & 33.15 & 0.05 & 33.22 & 33.03 & 672 \\
\hline ST1 & $\mathrm{VMCM}$ & 6.25 & $19950201 / 0000$ & $19950228 / 2300$ & 33.16 & 0.04 & 33.22 & 33.05 & 672 \\
\hline ST2 & SCAT & 1.00 & $19950203 / 2100$ & $19950228 / 2300$ & 33.17 & 0.03 & 33.22 & 33.06 & 603 \\
\hline ST2 & TPOD & 5.40 & $19950203 / 2100$ & $19950228 / 2300$ & 33.17 & 0.02 & 33.21 & 33.05 & 603 \\
\hline ST2 & SCAT & 11.00 & $19950203 / 2100$ & $19950228 / 2300$ & 33.17 & 0.02 & 33.21 & 33.08 & 603 \\
\hline ST2 & $\mathrm{VMCM}$ & 17.00 & $19950204 / 0000$ & 1995 0228/2300 & 33.15 & 0.02 & 33.20 & 33.07 & 600 \\
\hline $\mathrm{CR}$ & SBTC & 2.00 & 一 & - & - & - & - & - & - \\
\hline CR & SBTC & 10.00 & - & - & - & - & - & - & - \\
\hline SF & SBTC & 1.00 & 1995 0201/0000 & $19950210 / 2300$ & 33.05 & 0.07 & 33.20 & 32.89 & 237 \\
\hline $\mathrm{SF}$ & SBTC & 5.00 & 1995 0201/0000 & $19950228 / 2300$ & 33.10 & 0.09 & 33.21 & 32.85 & 672 \\
\hline $\mathrm{SF}$ & SBTC & 10.00 & $19950201 / 0000$ & $19950228 / 2300$ & 33.10 & 0.09 & 33.21 & 32.86 & 672 \\
\hline $\mathrm{SF}$ & SBTC & 15.00 & - & - & - & - & - & - & - \\
\hline $\mathrm{SF}$ & SBTC & 20.00 & $19950201 / 0000$ & $19950228 / 2300$ & 33.10 & 0.08 & 33.21 & 32.91 & 672 \\
\hline $\mathrm{SF}$ & SBTC & 25.00 & $19950201 / 0000$ & $19950228 / 2300$ & 33.11 & 0.07 & 33.21 & 32.91 & 672 \\
\hline SF & SBTC & 30.00 & 1995 0201/0000 & $19950228 / 2300$ & 33.12 & 0.07 & 33.21 & 32.91 & 672 \\
\hline $\mathrm{SF}$ & SBTC & 35.00 & $19950201 / 0000$ & $0228 / 2300$ & 33.12 & 0.07 & 33.21 & 32.91 & 672 \\
\hline $\mathrm{SF}$ & SBTC & 40.00 & 1995 0201/0000 & $19950228 / 2300$ & 33.12 & 0.07 & 33.21 & 32.91 & 672 \\
\hline SF & SBTC & 45.00 & $19950201 / 0000$ & $19950227 / 1500$ & 33.13 & 0.07 & 33.23 & 32.94 & 640 \\
\hline NP & $\mathrm{RCM}$ & 23.00 & $19950201 / 0000$ & $19950228 / 2300$ & 32.80 & 0.19 & 33.21 & 32.40 & 672 \\
\hline \multicolumn{10}{|c|}{ March 1995 Salinity (PSU) } \\
\hline ST1 & IMET & 1.00 & $19950301 / 0000$ & $19950331 / 2300$ & & 0.21 & & 32.32 & 744 \\
\hline ST1 & VAWR & 1.50 & $19950301 / 0000$ & $19950331 / 2300$ & & 0.21 & 33. & 32.32 & 744 \\
\hline ST1 & SCAT & 2.00 & $19950301 / 0000$ & $19950331 / 2300$ & 32.69 & 0.21 & 33.15 & 32.32 & 744 \\
\hline ST1 & TPOD & 3.50 & $19950301 / 0000$ & $19950331 / 2300$ & 32.73 & 0.20 & 33.16 & 32.37 & 744 \\
\hline ST1 & MTR & 5.65 & $19950301 / 0000$ & $19950331 / 2300$ & 32.81 & 0.16 & 33.17 & 32.53 & 744 \\
\hline ST1 & $\mathrm{VMCM}$ & 6.25 & $19950301 / 0000$ & $19950331 / 2300$ & 32.81 & 0.17 & 33.16 & 32.54 & 744 \\
\hline ST2 & SCAT & 1.00 & $19950301 / 0000$ & $19950331 / 2300$ & 32.77 & 0.18 & & 32.42 & 744 \\
\hline $\mathrm{ST} 2$ & TPOD & 5.40 & $19950301 / 0000$ & $19950331 / 2300$ & 32.77 & 0.18 & 33.17 & 32.44 & 744 \\
\hline ST2 & SCAT & 11.00 & $19950301 / 0000$ & $19950331 / 2300$ & 32.82 & 0.17 & 33.18 & 32.51 & 744 \\
\hline ST2 & $\mathrm{VMCM}$ & 17.00 & $19950301 / 0000$ & $19950331 / 2300$ & 32.82 & 0.17 & 33.17 & 32.49 & 744 \\
\hline $\mathrm{CR}$ & SBTC & 2.00 & - & - & - & - & - & - & - \\
\hline CR & SBTC & 10.00 & 一 & - & - & - & - & - & - \\
\hline
\end{tabular}


Table 21: Monthly Salinity Hourly-Averaged Statistics

\begin{tabular}{|c|c|c|c|c|c|c|c|c|c|}
\hline Sta & $\begin{array}{l}\text { Inst } \\
\text { Type } \\
(\mathbf{m})\end{array}$ & $\begin{array}{l}\text { Sensor } \\
\text { Height } \\
\quad(\mathbf{m})\end{array}$ & $\begin{array}{c}\text { GMT } \\
\text { Start Time } \\
(\mathbf{y ~ m ~ d / h ~ m ) ~}\end{array}$ & $\begin{array}{c}\text { GMT } \\
\text { Stop Time } \\
(\text { y } \mathbf{m ~ d} / \mathbf{h ~ m})\end{array}$ & Mean & $\begin{array}{l}\text { Std } \\
\text { Dev }\end{array}$ & Max & Min & Npts \\
\hline \multicolumn{10}{|c|}{ March 1995 Salinity (PSU) (Continued) } \\
\hline SF & SBTC & 1.00 & - & - & - & - & - & - & - \\
\hline $\mathrm{SF}$ & SBTC & 5.00 & $19950301 / 0000$ & $19950304 / 1500$ & 33.10 & 0.05 & 33.15 & 32.94 & 88 \\
\hline SF & SBTC & 10.00 & $19950301 / 0000$ & $19950304 / 1500$ & 33.10 & 0.05 & 33.15 & 32.95 & 88 \\
\hline $\mathrm{SF}$ & SBTC & 15.00 & - & - & - & - & 一 & - & - \\
\hline $\mathrm{SF}$ & SBTC & 20.00 & $19950301 / 0000$ & $19950304 / 1500$ & 33.11 & 0.04 & 33.14 & 32.98 & 88 \\
\hline SF & SBTC & 25.00 & $19950301 / 0000$ & $19950304 / 1500$ & & 0.03 & 33.15 & 33.02 & 88 \\
\hline $\mathrm{SF}$ & SBTC & 30.00 & $19950301 / 0000$ & $19950304 / 1500$ & 33.13 & 0.03 & 33.16 & 33.05 & 88 \\
\hline SF & SBTC & 35.00 & $19950301 / 0000$ & $19950304 / 1500$ & 33.13 & 0.03 & 33.16 & 33.06 & 88 \\
\hline $\mathrm{SF}$ & SBTC & 40.00 & $19950301 / 0000$ & $19950304 / 1500$ & 33.15 & 0.02 & 33.17 & 33.08 & 88 \\
\hline $\mathrm{SF}$ & SBTC & 45.00 & - & - & & 一 & - & - & - \\
\hline NP & $\mathrm{RCM}$ & 23.00 & $19950301 / 0000$ & $19950331 / 2300$ & 32.57 & 0.10 & 32.83 & 32.34 & 744 \\
\hline \multicolumn{10}{|c|}{ April 1995 Salinity (PSU) } \\
\hline ST1 & IMET & 1.00 & $19950401 / 0000$ & $19950430 / 2300$ & 32.61 & 0.12 & 32.98 & 31.97 & 720 \\
\hline ST1 & VAWR & 1.50 & $19950401 / 0000$ & $19950430 / 2300$ & 32.62 & 0.09 & 32.81 & 32.38 & 720 \\
\hline ST1 & SCAT & 2.00 & $19950401 / 0000$ & $19950430 / 2300$ & 32.63 & 0.09 & 32.81 & 32.42 & 720 \\
\hline ST1 & TPOD & 3.50 & $19950401 / 0000$ & $19950430 / 2300$ & 32.66 & 0.09 & 32.82 & 32.44 & 720 \\
\hline ST1 & MTR & 5.65 & $19950401 / 0000$ & $19950430 / 2300$ & 32.68 & 0.07 & 32.82 & 32.53 & 720 \\
\hline ST1 & $\mathrm{VMCM}$ & 6.25 & $19950401 / 0000$ & $19950430 / 2300$ & 32.70 & 0.07 & 32.94 & 32.56 & 720 \\
\hline ST2 & SCAT & 1.00 & $19950401 / 0000$ & $19950430 / 2300$ & 32.68 & 0.08 & 32.90 & 32.23 & 720 \\
\hline ST2 & TPOD & 5.40 & $19950401 / 0000$ & $19950430 / 2300$ & 32.69 & 0.06 & 32.89 & 32.58 & 720 \\
\hline $\mathrm{ST} 2$ & SCAT & 11.00 & $19950401 / 0000$ & $19950430 / 2300$ & 32.73 & 0.07 & 32.94 & 32.60 & 720 \\
\hline ST2 & $\mathrm{VMCM}$ & 17.00 & $19950401 / 0000$ & $19950430 / 2300$ & 32.71 & 0.07 & 32.92 & 32.59 & 720 \\
\hline $\mathrm{CR}$ & SBTC & 2.00 & $19950402 / 0400$ & $19950430 / 2300$ & 33.10 & 0.09 & 33.24 & 32.91 & 692 \\
\hline $\mathrm{CR}$ & SBTC & 10.00 & $19950402 / 0400$ & $19950430 / 2300$ & 33.11 & 0.08 & 33.24 & 32.92 & 692 \\
\hline SF & SBTC & & $19950426 / 1900$ & $19950430 / 2300$ & & & & & 106 \\
\hline $\mathrm{SF}$ & SBTC & 5.00 & $19950426 / 1900$ & $19950430 / 2300$ & 32.45 & 0.19 & 32.81 & 32.22 & 106 \\
\hline $\mathrm{SF}$ & SBTC & 10.00 & $19950426 / 1900$ & $19950430 / 2300$ & 32.59 & 0.21 & 32.81 & 32.23 & 106 \\
\hline $\mathrm{SF}$ & SBTC & 15.00 & $19950426 / 1900$ & $19950430 / 2300$ & 32.70 & 0.16 & 32.82 & 32.27 & 106 \\
\hline SF & SBTC & 20.00 & $19950426 / 1900$ & $19950430 / 2300$ & 32.75 & 0.08 & 32.82 & 32.50 & 106 \\
\hline $\mathrm{SF}$ & SBTC & 25.00 & 1995 0426/1900 & $19950430 / 2300$ & 32.76 & 0.05 & 32.82 & 32.67 & 106 \\
\hline $\mathrm{SF}$ & SBTC & 30.00 & $19950426 / 1900$ & $19950430 / 2300$ & 32.77 & 0.05 & 32.82 & 32.68 & 106 \\
\hline $\mathrm{SF}$ & SBTC & 35.00 & - & - & - & - & - & - & - \\
\hline $\mathrm{SF}$ & SBTC & 40.00 & $19950426 / 1900$ & $19950430 / 2300$ & 32.77 & 0.05 & 32.82 & 32.68 & 106 \\
\hline $\mathrm{SF}$ & SBTC & 45.00 & $19950426 / 1900$ & $19950430 / 2300$ & 32.79 & 0.05 & 32.84 & 32.70 & 106 \\
\hline NP & RCM & 23.00 & $19950401 / 0000$ & $19950409 / 2300$ & 32.59 & 0.10 & 32.75 & 32.14 & 216 \\
\hline
\end{tabular}


Table 21: Monthly Salinity Hourly-Averaged Statistics

\begin{tabular}{|c|c|c|c|c|c|c|c|c|c|}
\hline Sta & $\begin{array}{l}\text { Inst } \\
\text { Type } \\
(\mathrm{m})\end{array}$ & $\begin{array}{l}\text { Sensor } \\
\text { Height } \\
\quad(\mathrm{m})\end{array}$ & $\begin{array}{c}\text { GMT } \\
\text { Start Time } \\
(\mathbf{y ~} \mathbf{m ~ d} / \mathbf{h ~ m})\end{array}$ & $\begin{array}{c}\text { GMT } \\
\text { Stop Time } \\
(\mathbf{y} \mathbf{m ~ d} / \mathbf{h ~ m})\end{array}$ & Mean & $\begin{array}{l}\text { Std } \\
\text { Dev }\end{array}$ & Max & Min & Npts \\
\hline \multicolumn{10}{|c|}{ May 1995 Salinity (PSU) } \\
\hline ST1 & IMET & 1.00 & $19950501 / 0000$ & $19950531 / 2300$ & 32.64 & 0.28 & 34.28 & 32.28 & 744 \\
\hline ST1 & VAWR & 1.50 & $19950501 / 0000$ & $19950531 / 2300$ & & 0.30 & 34.37 & 32.29 & 744 \\
\hline ST1 & SCAT & 2.00 & $19950501 / 0000$ & $19950531 / 2300$ & 32.69 & 0.33 & 34.50 & 32.30 & 744 \\
\hline ST1 & TPOD & 3.50 & $19950501 / 0000$ & $19950531 / 2300$ & & & 34.64 & 32.38 & 744 \\
\hline ST1 & MTR & 5.65 & $19950501 / 0000$ & $19950531 / 2300$ & & 0.66 & 34.87 & 32.48 & 744 \\
\hline ST1 & $\mathrm{VMCM}$ & 6.25 & $19950501 / 0000$ & $19950531 / 2300$ & 33.54 & 0.76 & 35.26 & 32.52 & 744 \\
\hline $\mathrm{ST} 2$ & SCAT & 1.00 & $19950501 / 0000$ & $19950531 / 2300$ & 32.61 & 0.13 & & 32.20 & 744 \\
\hline $\mathrm{ST} 2$ & TPOD & 5.40 & $19950501 / 0000$ & $19950531 / 2300$ & & 0.15 & & 32.28 & 744 \\
\hline ST2 & SCAT & 11.00 & $19950501 / 0000$ & $19950531 / 2300$ & 32.76 & 0.21 & 33.96 & 32.42 & 744 \\
\hline ST2 & $\mathrm{VMCM}$ & 17.00 & $19950501 / 0000$ & $19950531 / 2300$ & 32.88 & 0.34 & 34.32 & 32.49 & 744 \\
\hline CR & SBTC & 2.00 & $19950501 / 0000$ & & & 0.09 & & 32.66 & 744 \\
\hline $\mathrm{CR}$ & SBTC & 10.00 & $19950501 / 0000$ & $19950531 / 2300$ & & 0.08 & 33.06 & 32.71 & 744 \\
\hline $\mathrm{SF}$ & SBTC & 1.00 & $19950501 / 0000$ & $19950531 / 2300$ & 32.63 & 0.28 & 33.95 & 32.18 & 744 \\
\hline $\mathrm{SF}$ & SBTC & 5.00 & $501 / 0000$ & 199 & & 2 & & 32.18 & 744 \\
\hline SF & SBTC & 10.00 & $501 / 0000$ & $531 / 2300$ & 32.70 & 0.35 & 34.73 & 32.20 & 744 \\
\hline SF & SBTC & 15.00 & $19950501 / 0000$ & $19950531 / 2300$ & 73 & 0.37 & 34. & 32.26 & 744 \\
\hline $\mathrm{SF}$ & SBTC & 20.00 & $19950501 / 0000$ & $531 / 2300$ & 32.81 & 0.40 & 34.88 & 32.27 & 744 \\
\hline $\mathrm{SF}$ & SBTC & 25.00 & $19950501 / 0000$ & $19950531 / 2300$ & 88 & 0.44 & 34.95 & 32.33 & 744 \\
\hline $\mathrm{SF}$ & SBTC & 30.00 & $19950501 / 0000$ & $19950531 / 2300$ & 32.95 & 0.49 & 34.94 & 32.40 & 744 \\
\hline $\mathrm{SF}$ & SBTC & 35.00 & - & - & - & - & 一 & - & - \\
\hline SF & SBTC & 40.00 & $19950501 / 0000$ & $19950531 / 2300$ & & 0.59 & 34.91 & 32.42 & 744 \\
\hline $\mathrm{SF}$ & SBTC & 45.00 & $19950501 / 0000$ & $19950531 / 2300$ & 33.18 & 0.62 & 34.95 & 32.45 & 744 \\
\hline NP & $\mathrm{RCM}$ & 23.00 & - & - & - & - & - & - & - \\
\hline \multicolumn{10}{|c|}{ June 1995 Salinity (PSU) } \\
\hline ST1 & IMET & & & & & 0.07 & & 32.07 & 720 \\
\hline ST1 & VAWR & 1.50 & $19950601 / 0000$ & $19950630 / 2300$ & & 0.04 & 32.60 & 32.29 & 720 \\
\hline ST1 & SCAT & 2.00 & $19950601 / 0000$ & $19950630 / 2300$ & & 0.04 & & 32.31 & 720 \\
\hline ST1 & TPOD & 3.50 & $19950601 / 0000$ & $19950630 / 2300$ & 32.58 & 0.05 & 32.73 & 32.42 & 720 \\
\hline ST1 & MTR & 5.65 & 1995 0601/0000 & $19950630 / 2300$ & 32.62 & 0.08 & 32.91 & 32.50 & 720 \\
\hline ST1 & $\mathrm{VMCM}$ & 6.25 & $19950601 / 0000$ & $19950630 / 2300$ & 32.63 & 0.08 & 32.91 & 32.51 & 720 \\
\hline ST2 & SCAT & 1.00 & $19950601 / 0000$ & & & 0.06 & & 32.16 & 720 \\
\hline ST2 & TPOD & 5.40 & $19950601 / 0000$ & $19950630 / 2300$ & $32.56^{\circ}$ & 0.05 & 32.72 & 32.42 & 720 \\
\hline ST2 & SCAT & 11.00 & $19950601 / 0000$ & $19950630 / 2300$ & 32.55 & 0.05 & 32.66 & 32.41 & 720 \\
\hline ST2 & $\mathrm{VMCM}$ & 17.00 & $19950601 / 0000$ & $19950630 / 2300$ & 32.55 & 0.05 & 32.66 & 32.46 & 720 \\
\hline CR & SBTC & & & & & & & 32.54 & 720 \\
\hline CR & SBTC & 10.00 & $19950601 / 0000$ & $19950630 / 2300$ & 32.66 & 0.06 & 32.78 & 32.54 & 720 \\
\hline
\end{tabular}


Table 21: Monthly Salinity Hourly-Averaged Statistics

\begin{tabular}{|c|c|c|c|c|c|c|c|c|c|}
\hline Sta & $\begin{array}{l}\text { Inst } \\
\text { Type } \\
(\mathbf{m})\end{array}$ & $\begin{array}{l}\text { Sensor } \\
\text { Height } \\
\quad(\mathrm{m})\end{array}$ & $\begin{array}{c}\text { GMT } \\
\text { Start Time } \\
(\mathbf{y} \mathbf{m ~ d} / \mathbf{h ~ m})\end{array}$ & $\begin{array}{c}\text { GMT } \\
\text { Stop Time } \\
(\mathbf{y ~ m ~ d / h ~ m ) ~}\end{array}$ & Mean & $\begin{array}{l}\text { Std } \\
\text { Dev }\end{array}$ & $\operatorname{Max}$ & Min & Npts \\
\hline \multicolumn{10}{|c|}{ June 1995 Salinity (PSU) (Continued) } \\
\hline $\mathrm{SF}$ & SBTC & 1.00 & $19950601 / 0000$ & $19950630 / 2300$ & 32.48 & 0.06 & 32.58 & 32.25 & 720 \\
\hline $\mathrm{SF}$ & SBTC & 5.00 & $19950601 / 0000$ & $19950630 / 2300$ & 32.50 & 0.04 & 32.60 & 32.32 & 720 \\
\hline $\mathrm{SF}$ & SBTC & 10.00 & $19950601 / 0000$ & $19950630 / 2300$ & 32.51 & 0.04 & 32.66 & 32.35 & 720 \\
\hline SF & SBTC & 15.00 & $19950601 / 0000$ & $19950630 / 2300$ & 32.57 & 0.04 & 32.69 & 32.41 & 720 \\
\hline SF & SBTC & 20.00 & $19950601 / 0000$ & $19950630 / 2300$ & 32.55 & 0.05 & 32.69 & 32.44 & 720 \\
\hline $\mathrm{SF}$ & SBTC & 25.00 & $19950601 / 0000$ & $19950630 / 2300$ & 32.57 & 0.05 & 32.71 & 32.34 & 720 \\
\hline $\mathrm{SF}$ & SBTC & 30.00 & $19950601 / 0000$ & $19950630 / 2300$ & 32.57 & 0.06 & 32.71 & 32.46 & 720 \\
\hline $\mathrm{SF}$ & SBTC & 35.00 & - & - & - & - & - & - & - \\
\hline SF & SBTC & 40.00 & $19950601 / 0000$ & $19950630 / 2300$ & 32.59 & 0.06 & 32.73 & 32.48 & 720 \\
\hline $\mathrm{SF}$ & SBTC & 45.00 & $19950601 / 0000$ & $19950630 / 2300$ & 32.61 & 0.06 & 32.75 & 32.49 & 720 \\
\hline NP & $\mathrm{RCM}$ & 23.00 & - & - & - & - & - & - & - \\
\hline \multicolumn{10}{|c|}{ July 1995 Salinity (PSU) } \\
\hline ST1 & IMET & 1.00 & $19950701 / 0000$ & $19950731 / 2300$ & 32.43 & 0.09 & 32.91 & 32.09 & 744 \\
\hline ST1 & VAWR & 1.50 & $19950701 / 0000$ & $19950731 / 2300$ & 32.46 & 0.10 & 33.20 & 32.29 & 744 \\
\hline ST1 & SCAT & 2.00 & $19950701 / 0000$ & $19950731 / 2300$ & 32.50 & 0.14 & 33.57 & 32.35 & 744 \\
\hline ST1 & TPOD & 3.50 & $19950701 / 0000$ & $19950731 / 2300$ & 32.56 & 0.08 & 33.18 & 32.46 & 744 \\
\hline $\mathrm{ST} 1$ & MTR & 5.65 & $19950701 / 0000$ & $19950731 / 2300$ & 32.59 & 0.05 & 32.78 & 32.50 & 744 \\
\hline ST1 & $\mathrm{VMCM}$ & 6.25 & $19950701 / 0000$ & $19950731 / 2300$ & 32.63 & 0.08 & 32.96 & 32.53 & 744 \\
\hline ST2 & SCAT & 1.00 & $19950701 / 0000$ & $19950731 / 2300$ & 32.39 & 0.07 & 32.69 & 31.92 & 744 \\
\hline $\mathrm{ST} 2$ & TPOD & 5.40 & $19950701 / 0000$ & $19950731 / 2300$ & 32.45 & 0.07 & 33.03 & 32.19 & 744 \\
\hline ST2 & $\mathrm{SCAT}$ & 11.00 & $19950701 / 0000$ & $19950731 / 2300$ & 32.45 & 0.02 & 32.58 & 32.38 & 744 \\
\hline ST2 & $\mathrm{VMCM}$ & 17.00 & $19950701 / 0000$ & $19950731 / 2300$ & 32.49 & 0.03 & 32.64 & 32.42 & 744 \\
\hline $\mathrm{CR}$ & SBTC & 2.00 & $19950701 / 0000$ & $19950731 / 2300$ & 32.48 & 0.05 & 32.60 & 32.35 & 744 \\
\hline CR & SBTC & 10.00 & $19950701 / 0000$ & $19950731 / 2300$ & 32.49 & 0.05 & 32.61 & 32.38 & 744 \\
\hline $\mathrm{SF}$ & SBTC & 1.00 & $19950701 / 0000$ & $19950731 / 2300$ & 32.39 & 0.07 & 32.95 & 32.23 & 744 \\
\hline $\mathrm{SF}$ & SBTC & 5.00 & $19950701 / 0000$ & $19950731 / 2300$ & 32.46 & 0.12 & 33.34 & 32.22 & 744 \\
\hline $\mathrm{SF}$ & SBTC & 10.00 & $19950701 / 0000$ & $19950731 / 2300$ & 32.50 & 0.14 & 33.38 & 32.31 & 744 \\
\hline SF & SBTC & 15.00 & $19950701 / 0000$ & 1995 0731/2300 & 32.56 & 0.13 & 33.53 & 32.43 & 744 \\
\hline $\mathrm{SF}$ & SBTC & 20.00 & $19950701 / 0000$ & 1995 0731/2300 & 32.53 & 0.09 & 33.28 & 32.41 & 744 \\
\hline SF & SBTC & 25.00 & $19950701 / 0000$ & $19950731 / 2300$ & 32.54 & 0.07 & 33.22 & 32.44 & 744 \\
\hline $\mathrm{SF}$ & SBTC & 30.00 & $19950701 / 0000$ & $19950731 / 2300$ & 32.54 & 0.04 & 32.92 & 32.46 & 744 \\
\hline $\mathrm{SF}$ & SBTC & 35.00 & - & - & - & - & - & - & - \\
\hline $\mathrm{SF}$ & SBTC & 40.00 & $19950701 / 0000$ & $19950731 / 2300$ & 32.57 & 0.05 & 32.74 & 32.46 & 744 \\
\hline $\mathrm{SF}$ & SBTC & 45.00 & $19950701 / 0000$ & $19950731 / 2300$ & 32.60 & 0.05 & 32.80 & 32.50 & 744 \\
\hline NP & $\mathrm{RCM}$ & 23.00 & - & - & - & - & - & - & - \\
\hline
\end{tabular}


Table 21: Monthly Salinity Hourly-Averaged Statistics

\begin{tabular}{|c|c|c|c|c|c|c|c|c|c|}
\hline Sta & $\begin{array}{l}\text { Inst } \\
\text { Type } \\
(\mathbf{m})\end{array}$ & $\begin{array}{l}\text { Sensor } \\
\text { Height } \\
\text { (m) }\end{array}$ & $\begin{array}{c}\text { GMT } \\
\text { Start Time } \\
(\mathbf{y ~ m ~ d / h ~ m ) ~}\end{array}$ & $\begin{array}{c}\text { GMT } \\
\text { Stop Time } \\
(\mathrm{y} \mathrm{m} \mathrm{d/h} \mathrm{m)}\end{array}$ & Mean & $\begin{array}{l}\text { Std } \\
\text { Dev }\end{array}$ & $\operatorname{Max}$ & Min & Npts \\
\hline \multicolumn{10}{|c|}{ August 1995 Salinity (PSU) } \\
\hline ST1 & IMET & 1.00 & $19950801 / 0000$ & $19950823 / 1600$ & 33.73 & 1.24 & 35.55 & 32.21 & 545 \\
\hline ST1 & VAWR & 1.50 & $19950801 / 0000$ & $19950823 / 1600$ & 33.87 & 1.23 & 35.55 & 32.28 & 545 \\
\hline ST1 & SCAT & 2.00 & $19950801 / 0000$ & $19950823 / 1600$ & 33.97 & 1.23 & 35.65 & 32.36 & 545 \\
\hline ST1 & TPOD & 3.50 & $19950801 / 0000$ & $19950823 / 1600$ & 33.64 & 1.13 & 35.79 & 32.38 & 545 \\
\hline ST1 & MTR & 5.65 & $19950801 / 0000$ & $19950817 / 0400$ & 32.79 & 0.25 & 34.39 & 32.50 & 389 \\
\hline ST1 & $\mathrm{VMCM}$ & 6.25 & $19950801 / 0000$ & $19950823 / 1600$ & 33.09 & 0.35 & 34.08 & 32.52 & 545 \\
\hline ST2 & SCAT & 1.00 & $19950801 / 0000$ & $19950804 / 2300$ & 32.35 & 0.02 & 32.41 & 32.29 & 96 \\
\hline ST2 & TPOD & 5.40 & $19950801 / 0000$ & $19950804 / 2300$ & 32.38 & 0.06 & 32.78 & 32.29 & 96 \\
\hline ST2 & SCAT & 11.00 & $19950801 / 0000$ & $19950804 / 2300$ & 32.52 & 0.10 & & 32.40 & 96 \\
\hline ST2 & VMCM & 17.00 & $19950801 / 0000$ & $19950804 / 2300$ & 32.57 & 0.07 & 32.71 & 32.44 & 96 \\
\hline $\mathrm{CR}$ & SBTC & & & & & 0.04 & 32.43 & 32.27 & 545 \\
\hline $\mathrm{CR}$ & SBTC & 10.00 & $19950801 / 0000$ & $19950823 / 1600$ & 32.38 & 0.02 & 32.42 & 32.33 & 545 \\
\hline SF & SBTC & 1.00 & $19950801 / 0000$ & $19950823 / 1600$ & 33.20 & 0.91 & 35.30 & 32.30 & 545 \\
\hline SF & SBTC & 5.00 & $19950801 / 0000$ & $19950823 / 1600$ & 33.34 & 1.00 & 35.60 & 32.31 & 545 \\
\hline SF & SBTC & 10.00 & $19950801 / 0000$ & $19950823 / 1600$ & 33.36 & 0.99 & 35.63 & 32.32 & 545 \\
\hline $\mathrm{SF}$ & SBTC & 15.00 & $19950801 / 0000$ & $19950823 / 1600$ & 33.23 & 0.85 & 35.44 & 32.32 & 545 \\
\hline $\mathrm{SF}$ & SBTC & 20.00 & $19950801 / 0000$ & $19950823 / 1600$ & 32.97 & 0.69 & 35.31 & 32.33 & 545 \\
\hline SF & SBTC & 25.00 & $19950801 / 0000$ & $19950823 / 1600$ & 32.81 & 0.55 & 35.51 & 32.35 & 545 \\
\hline $\mathrm{SF}$ & SBTC & 30.00 & $19950801 / 0000$ & $19950823 / 1600$ & 32.70 & 0.37 & 35.38 & 32.35 & 545 \\
\hline SF & SBTC & 35.00 & - & - & - & - & - & - & - \\
\hline SF & SBTC & 40.00 & $19950801 / 0000$ & $19950823 / 1600$ & 32.70 & 0.19 & 33.83 & 32.40 & 545 \\
\hline SF & SBTC & 45.00 & $19950801 / 0000$ & $19950823 / 1600$ & 32.76 & 0.19 & 33.53 & 32.46 & 545 \\
\hline NP & $\mathrm{rcm}$ & 23.00 & - & - & - & - & - & - & - \\
\hline
\end{tabular}





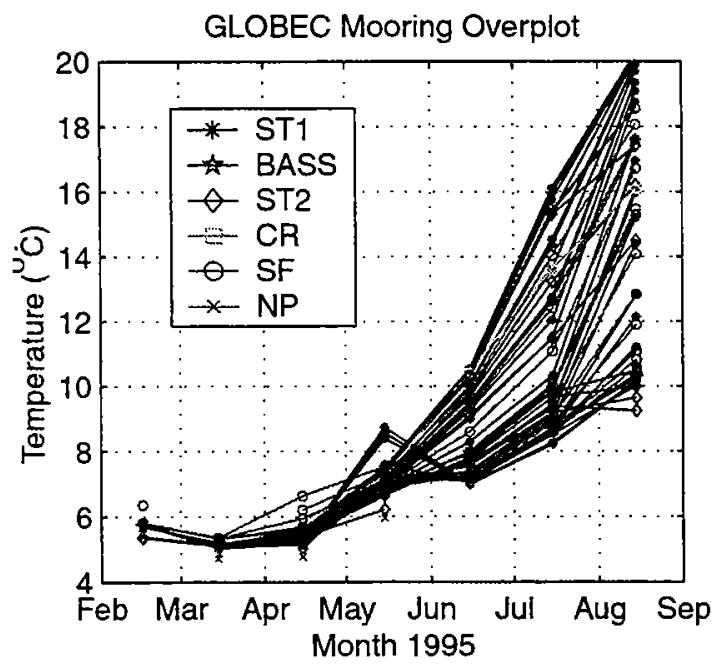

\section{Monthly Averages: \\ Water Temperature}

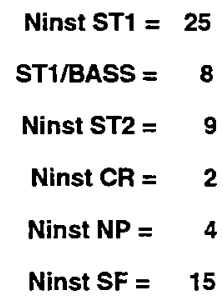

Crest and Northeast Peak Moorings

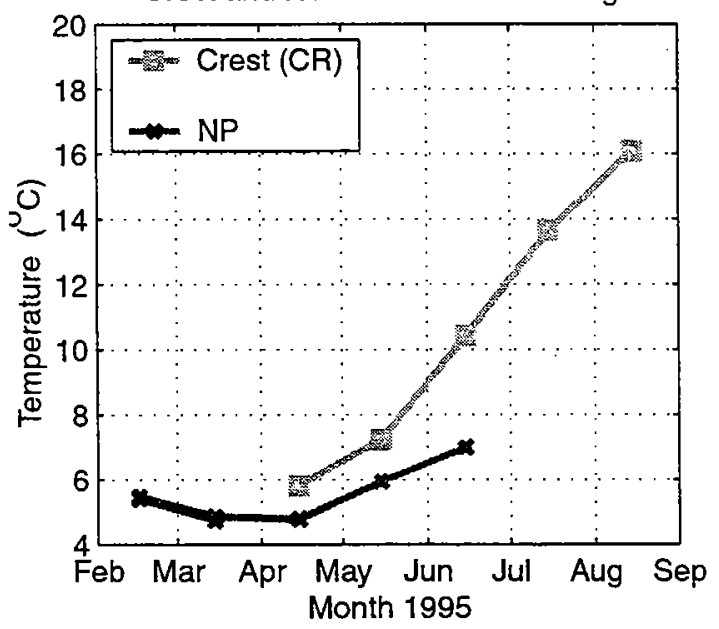

Southern Flank Mooring
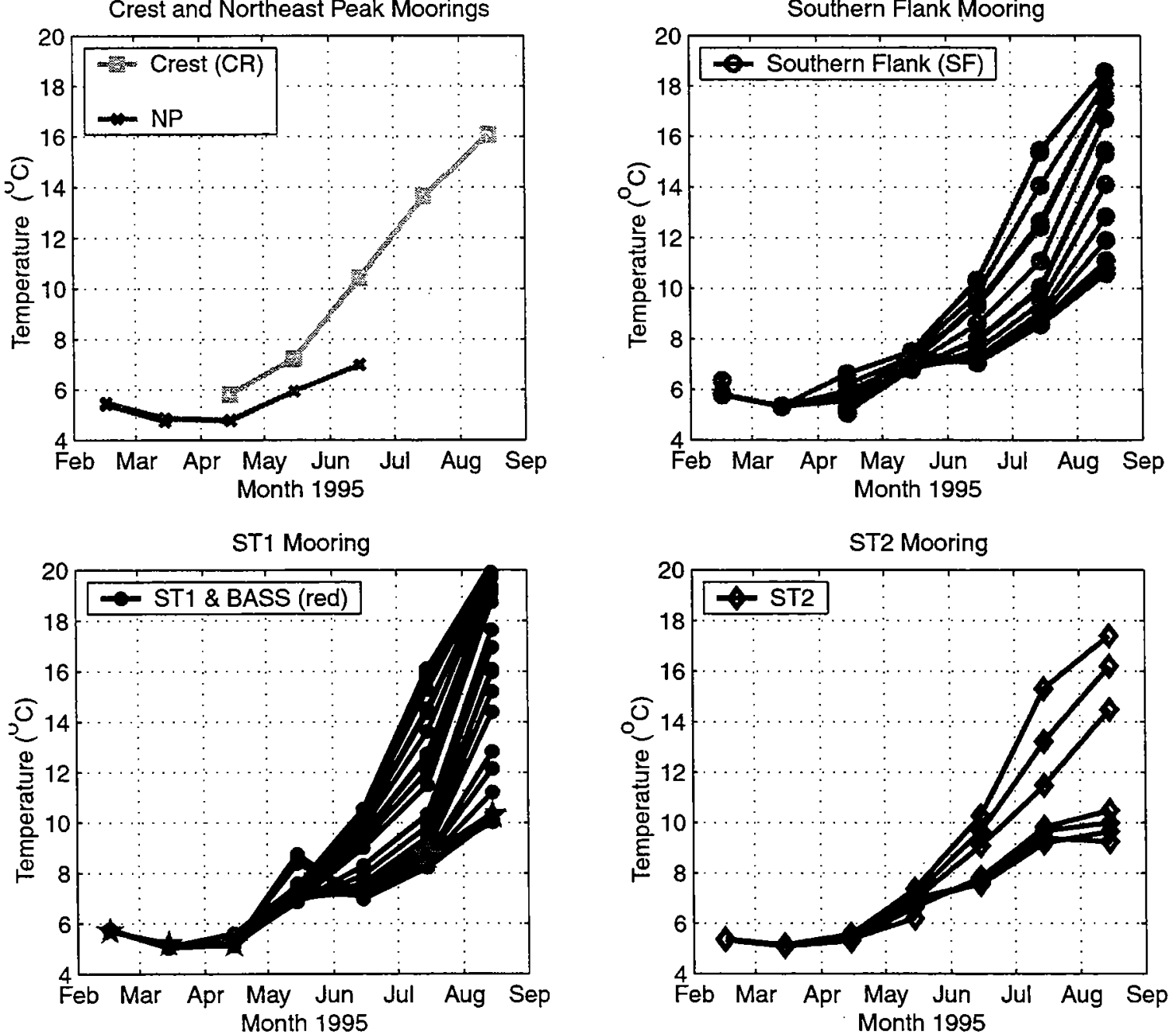

Figure 28: Plot of Monthly Means for Temperature 


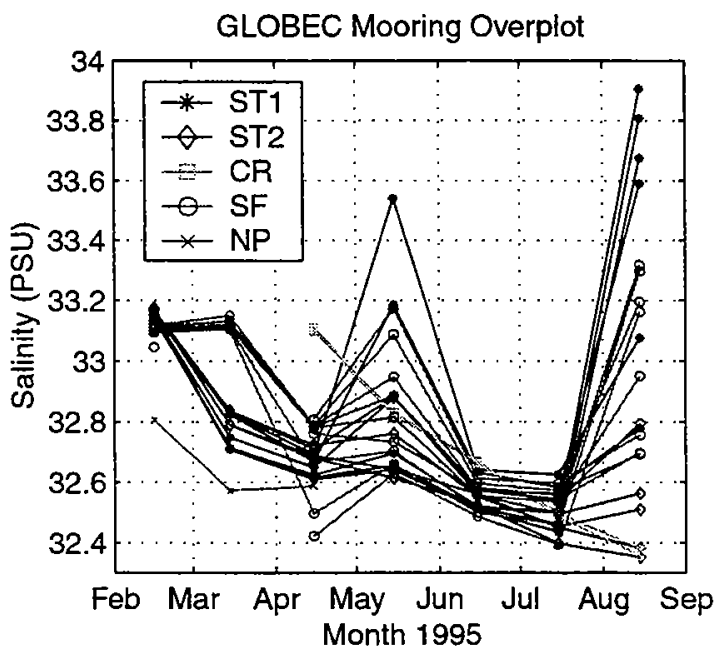

\section{Monthly Averages: Salinity (ppt)}
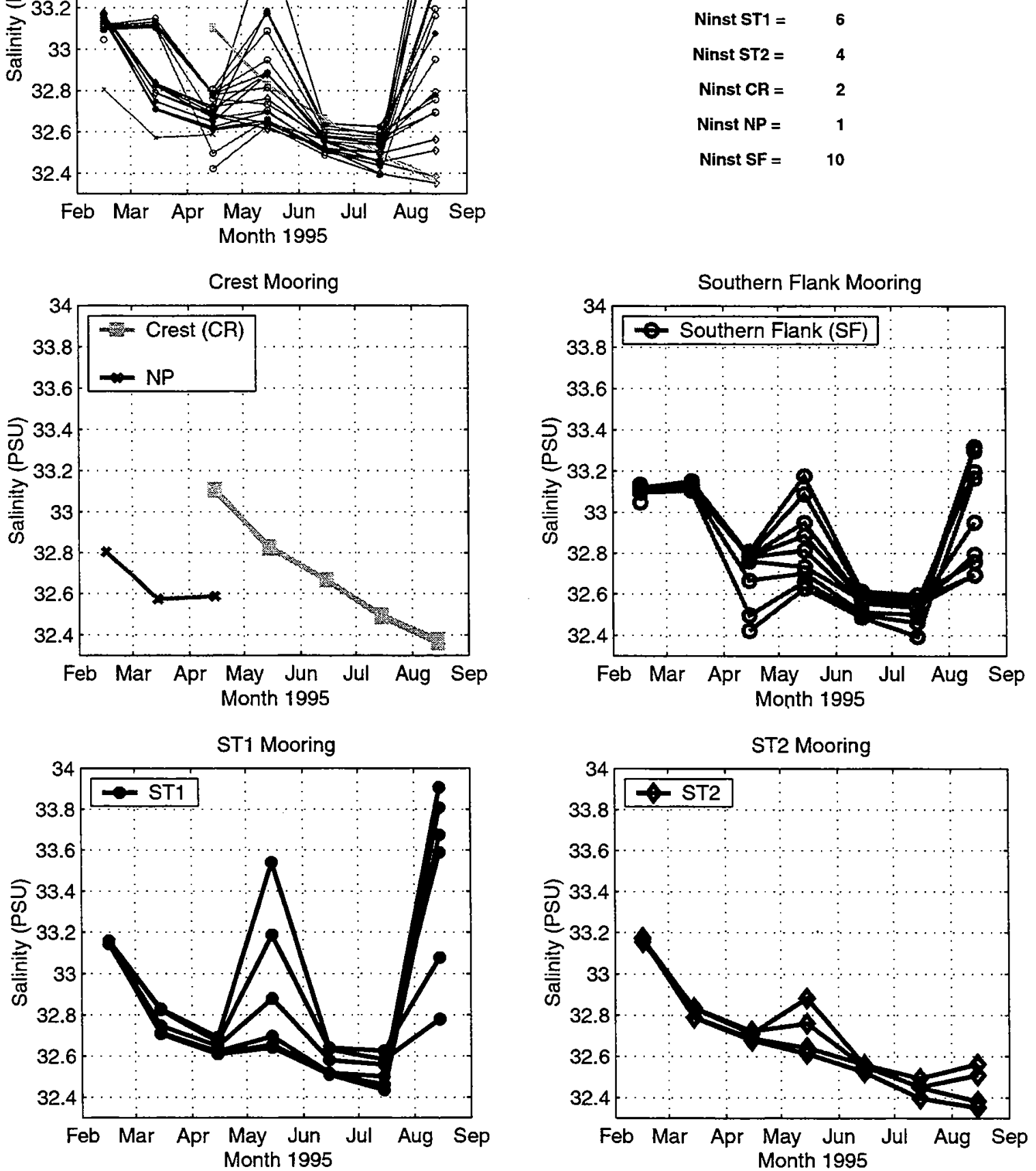

Figure 29: Plot of Monthly Means for Salinity 


\section{References}

Background Papers and Supporting Data on the Practical Salinity Scale 1978. UNESCO Technical Papers in Marine Science, 37, UNESCO, 1981.

Background Papers and Supporting Data on the International Equation of State of Seawater 1980. UNESCO Technical Papers in Marine Science, 38, UNESCO, 1981.

Beardsley, R. C., R. Limeburner, and L. K. Rosenfeld, 1985. Introduction to CODE-2 moored array and large-scale data report. In: CODE-2: Moored Array and Large-Scale Data Report, Richard Limeburner, editor; Woods Hole Oceanographic Institution Technical Report No. WHOI-85-35 pp. 1-21.

Buckley, L. J. and G. Lough, 1987. Recent growth, biochemical composition, and prey field of larval haddock (Melogrammus aeglefinus) and Atlantic cod (Gadus morhua) on Georges Bank. Canadian Journal of Fisheries and Aquatic Sciences, 44, 14-25.

Dean, J. P., and R. C. Beardsley, 1988. A vector-averaging wind recorder (VAWR) system for surface meteorological measurements in CODE. Woods Hole Oceanographic Institution Technical Report, WHOI-88-20, 68 pp.

GLOBEC, 1992. U.S. GLOBEC: Northwest Atlantic Implementation Plan. U.S. GLOBEC Report No. 6, June, 1992.

Hosom, D. S., R. A. Weller, R. E. Payne and K. E. Prada, 1995. The IMET (Improved METeorology) ship and buoy systems. Journal of Atmospheric and Oceanic Technology, 12(3), $527-540$.

Irish, J. D., and S. Kery, 1996. Elastic tether technology for shallow water moorings in harsh environments: Results from Georges Bank. Proceedings of Oceans '96, 635-639.

Irish, J. D. 1997. Elastic tether mooring technology: Experiences in the Gulf of Maine and on Georges Bank. Sea Technology, 38, 61-69.

Irish, J., 2000. Experience with the GLOBEC Long-Term Moorings on Georges Bank. Proceedings of ONR/MTS Buoy Workshop.

Paul, W., J. Irish, J. Gobat, and M. Grosenbaugh, 1999. Taut Elastomeric and Chain Catenary Surface Buoy Moorings. Proceedings Oceans'99, 418-426.

Tarbell, S. A., A. Spencer, and E. T. Montgomery, 1988. The Buoy Group Data Processing System. Woods Hole Oceanographic Institution Technical Memorandum, WHOI-3-88, 207 pp.

The Practical Salinity Scale 1978 and the International Equation of State of Seawater, 1980. UNESCO Technical Papers in Marine Science, 36, UNESCO, 1981.

Weller, R. A., D. L. Rudnick, R. E. Payne, J. P. Dean, N. J. Pennington and R. P. Trask, 1990. Measuring near-surface meteorology over the ocean from an array of surface moorings in the subtropical convergence zone. Journal of Atmospheric and Oceanic Technology, 7(1), 85-103. 
Williams, A. J., 3rd, J. S. Tochko, R. L. Koehler, W. D. Grant, T. F. Gross, and C.V.R. Dunn, 1987. Measurement of turbulence in the oceanic bottom boundary layer with an acoustic current meter array. Journal of Atmospheric and Oceanic Technology, 4, 312-327.

Werner, S. R., R. C. Beardsley, and A. J. Williams, et al., 2001. Bottom friction and bottom bedforms on the southern flank of Georges Bank. Journal of Geophysical Research, submitted.

Yang, D., B. Goodison, J. Metcalfe, V. Golubev, R. Bates, T. Pangburn, and C. Hanson, 1998. Accuracy of NWS $8^{\prime \prime}$-stand nonrecording precipitation gauge: results and application of WMO intercomparison. Journal of Atmospheric and Oceanic Technology, 15, 54-68.

Yuter, S. and W. Parker. Rainfall measurement on ship revisited: The 1997 PACS TEPPS cruise. Journal of Applied Meteorology, submitted in 2000. 


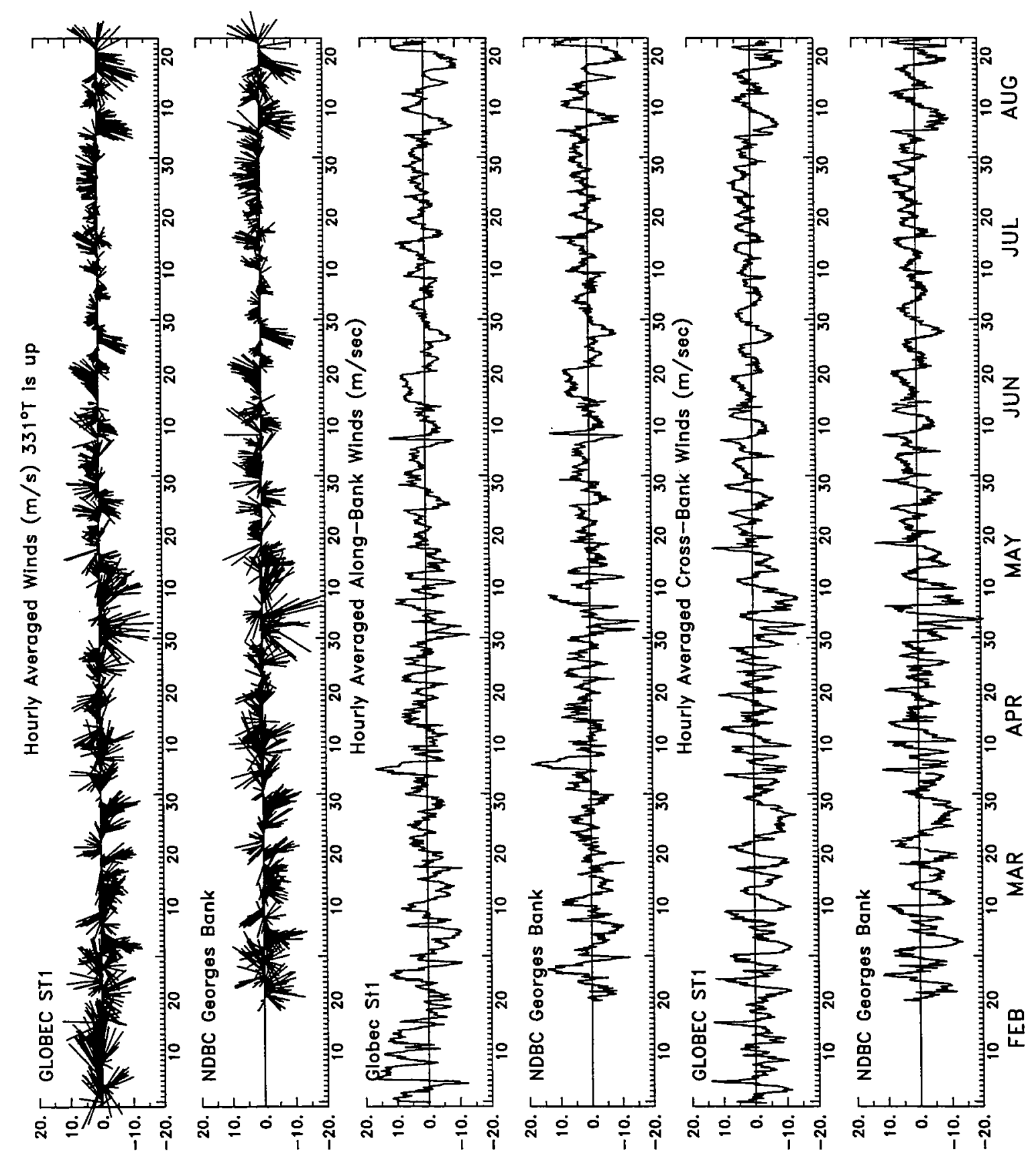

Figure 30 


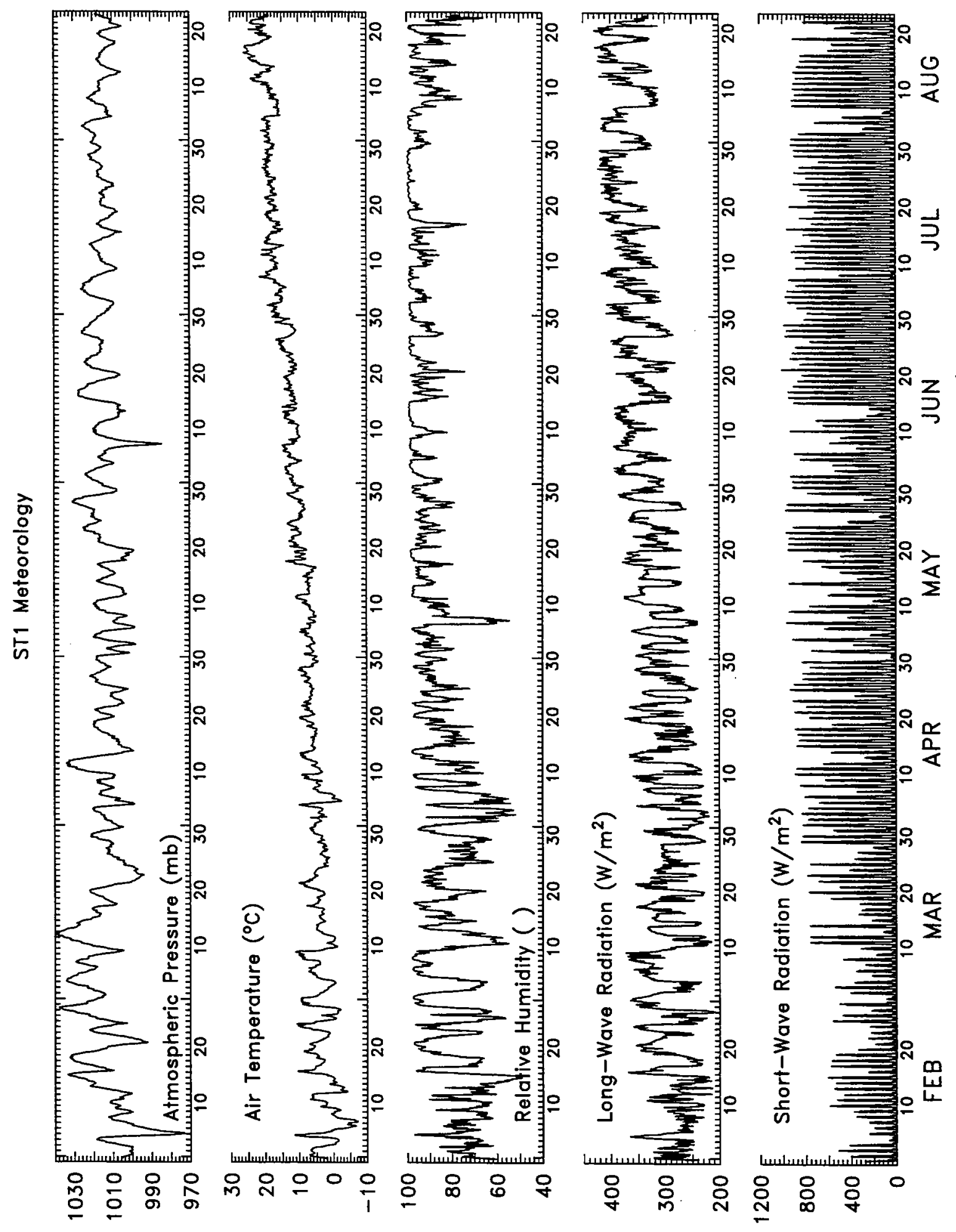

Figure 31 


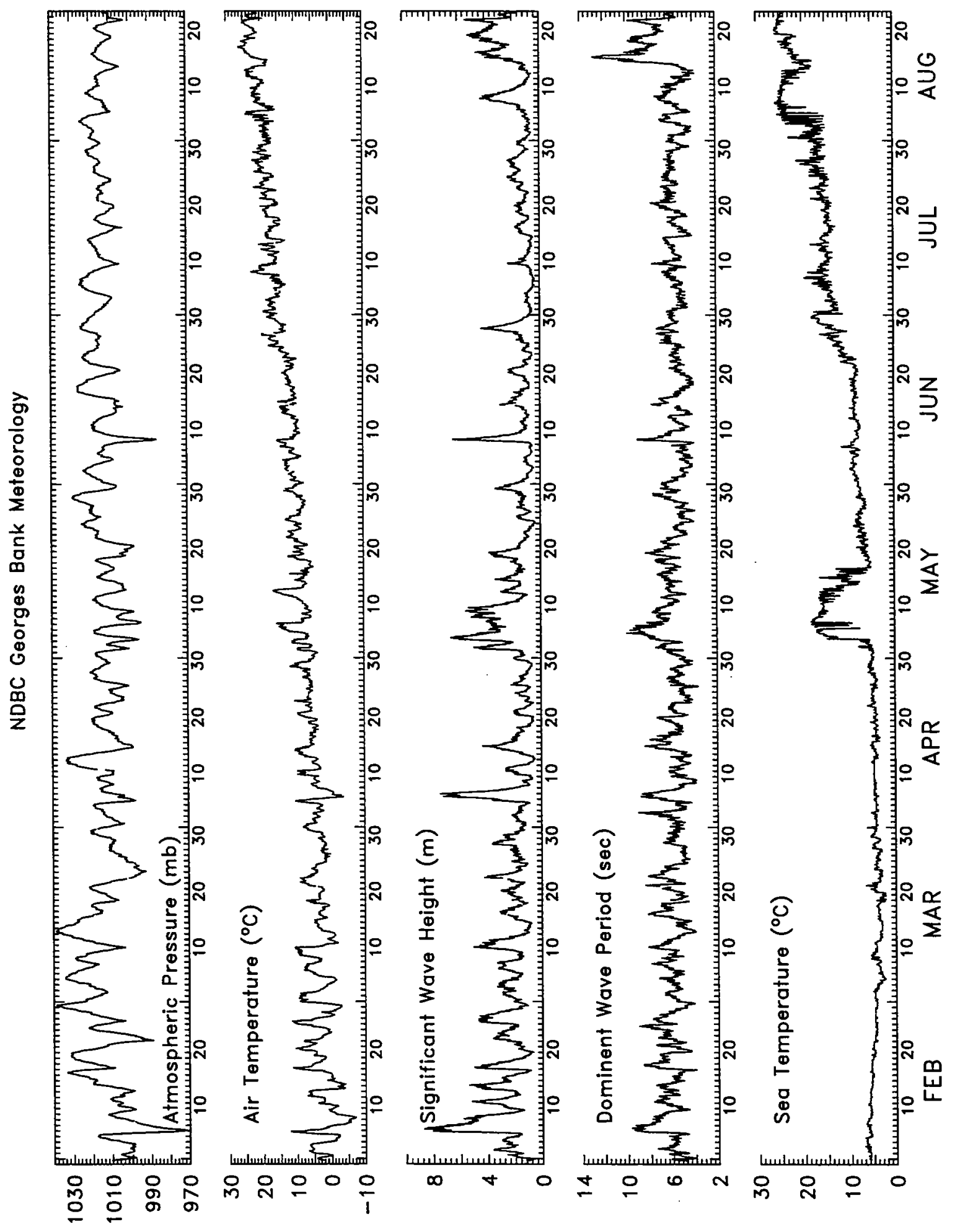

Figure 32 


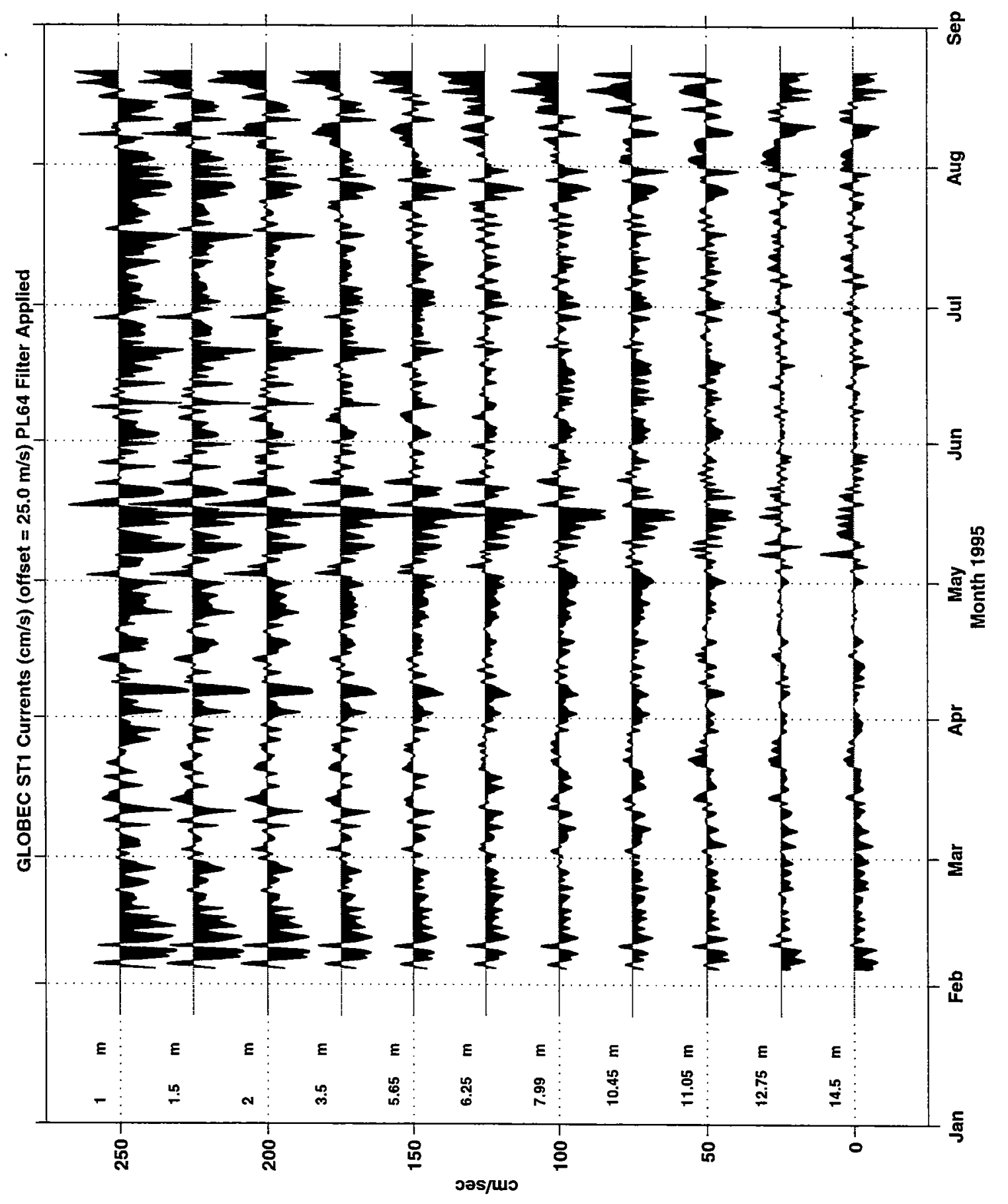

Figure 33 

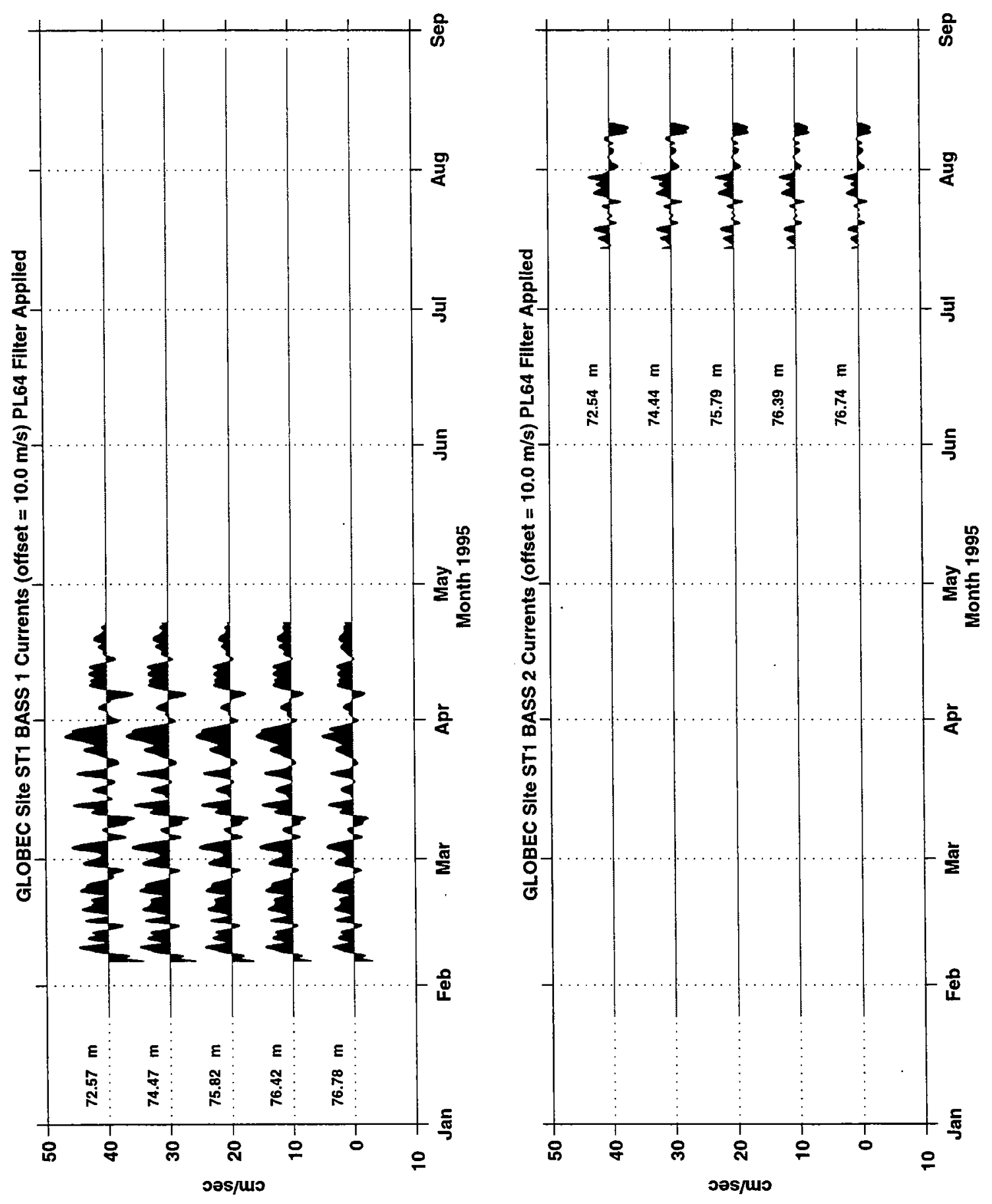

Figure 34 

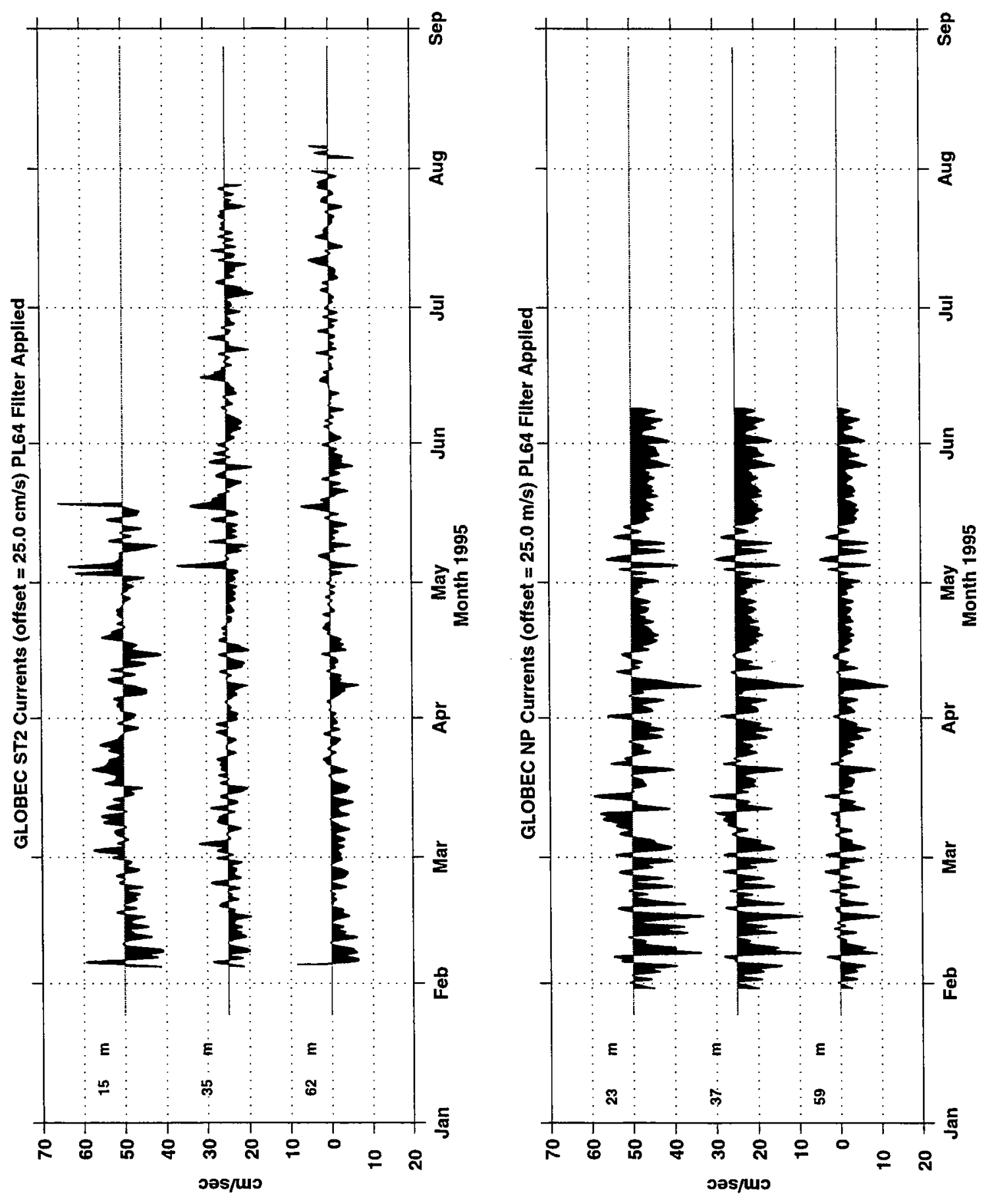

Figure 35 


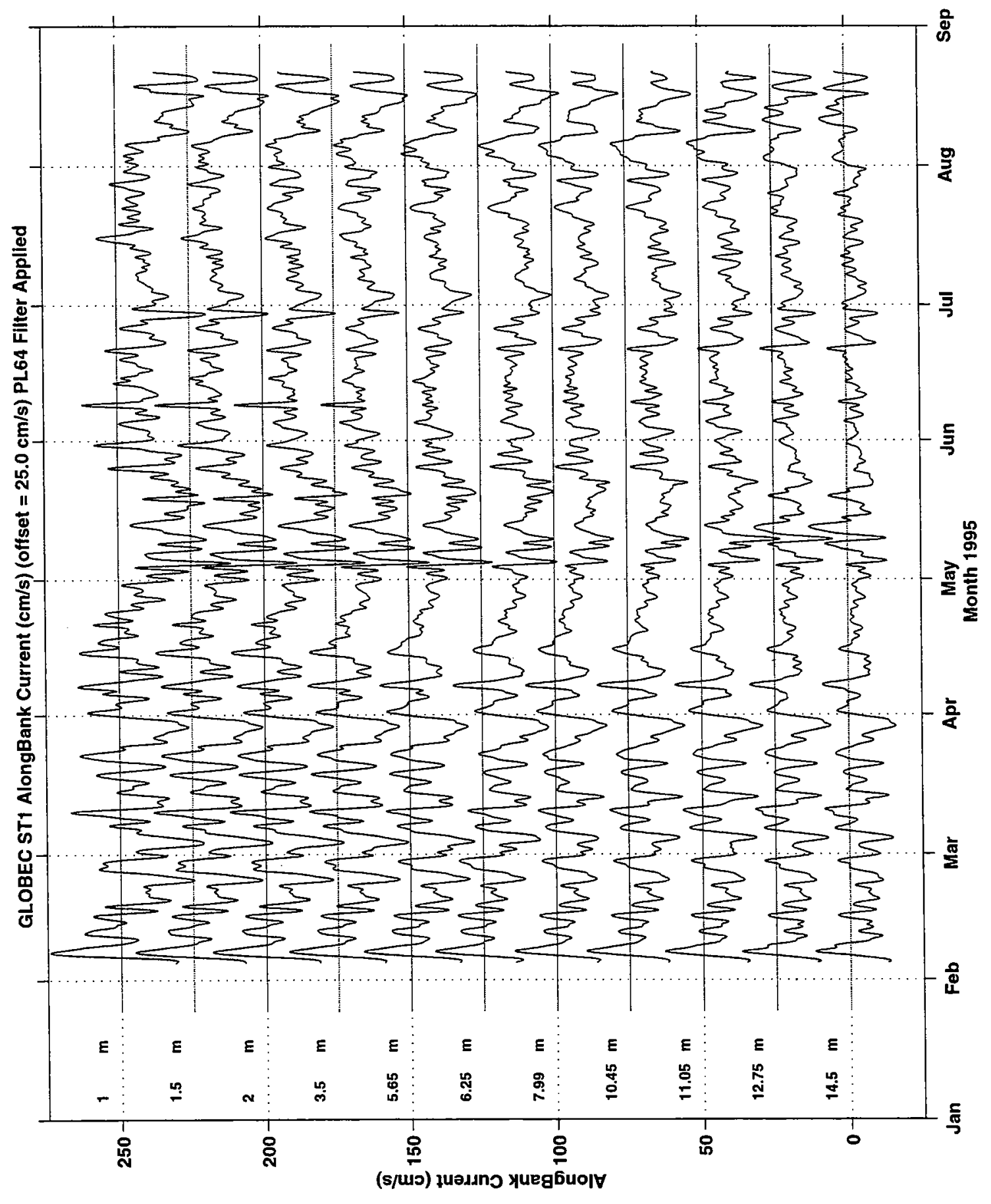

Figure 36 


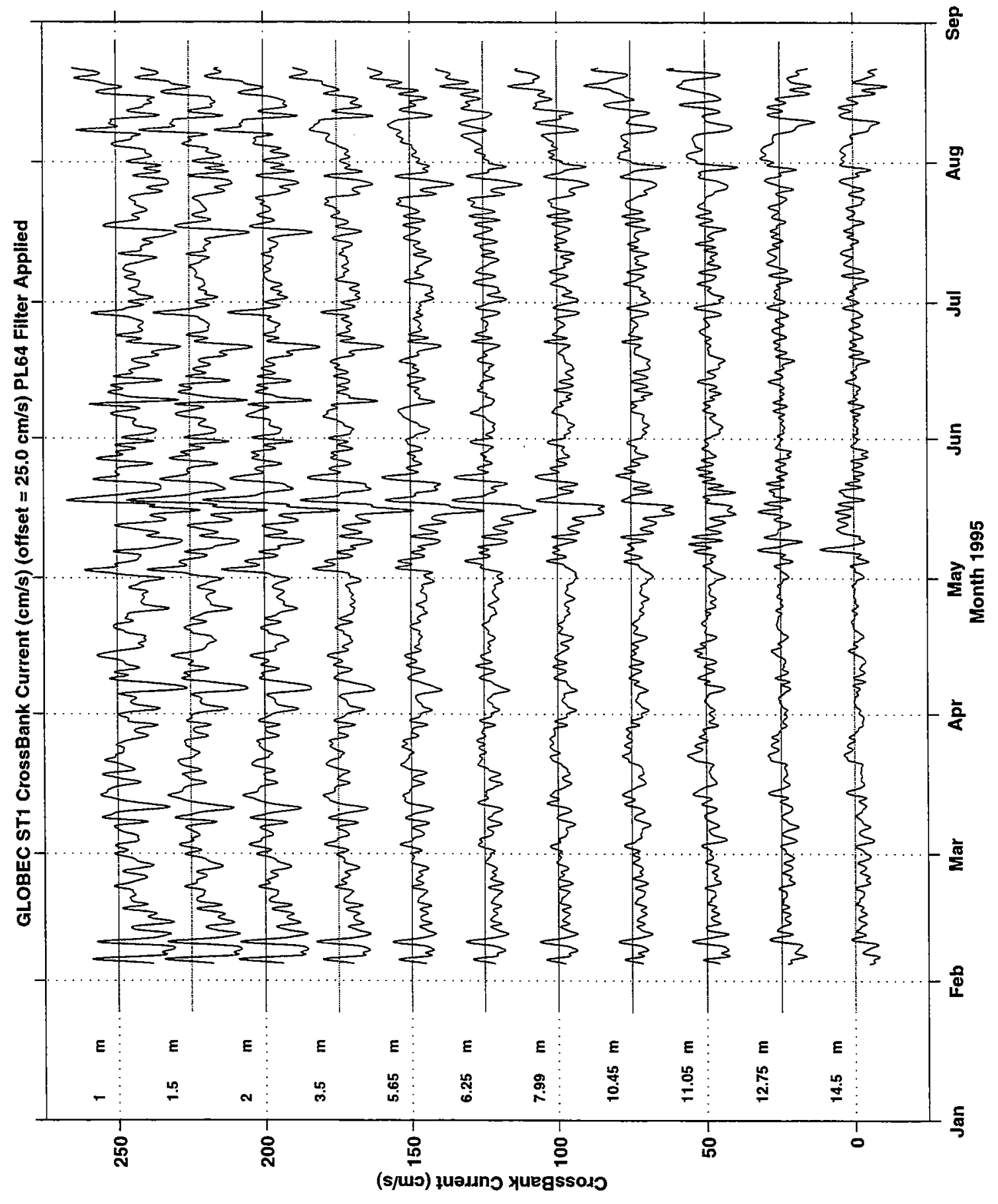

Figure 37 

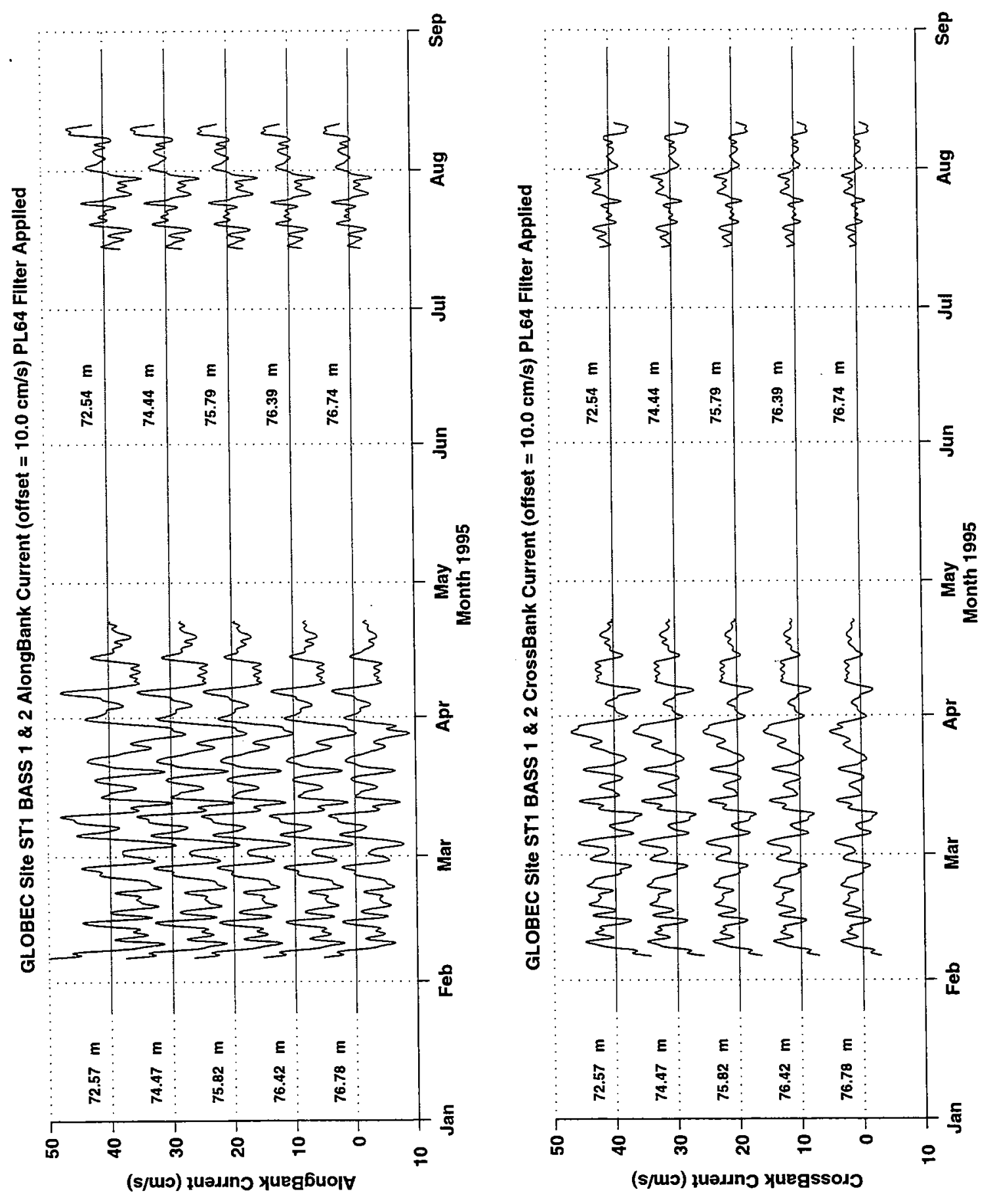

Figure 38 

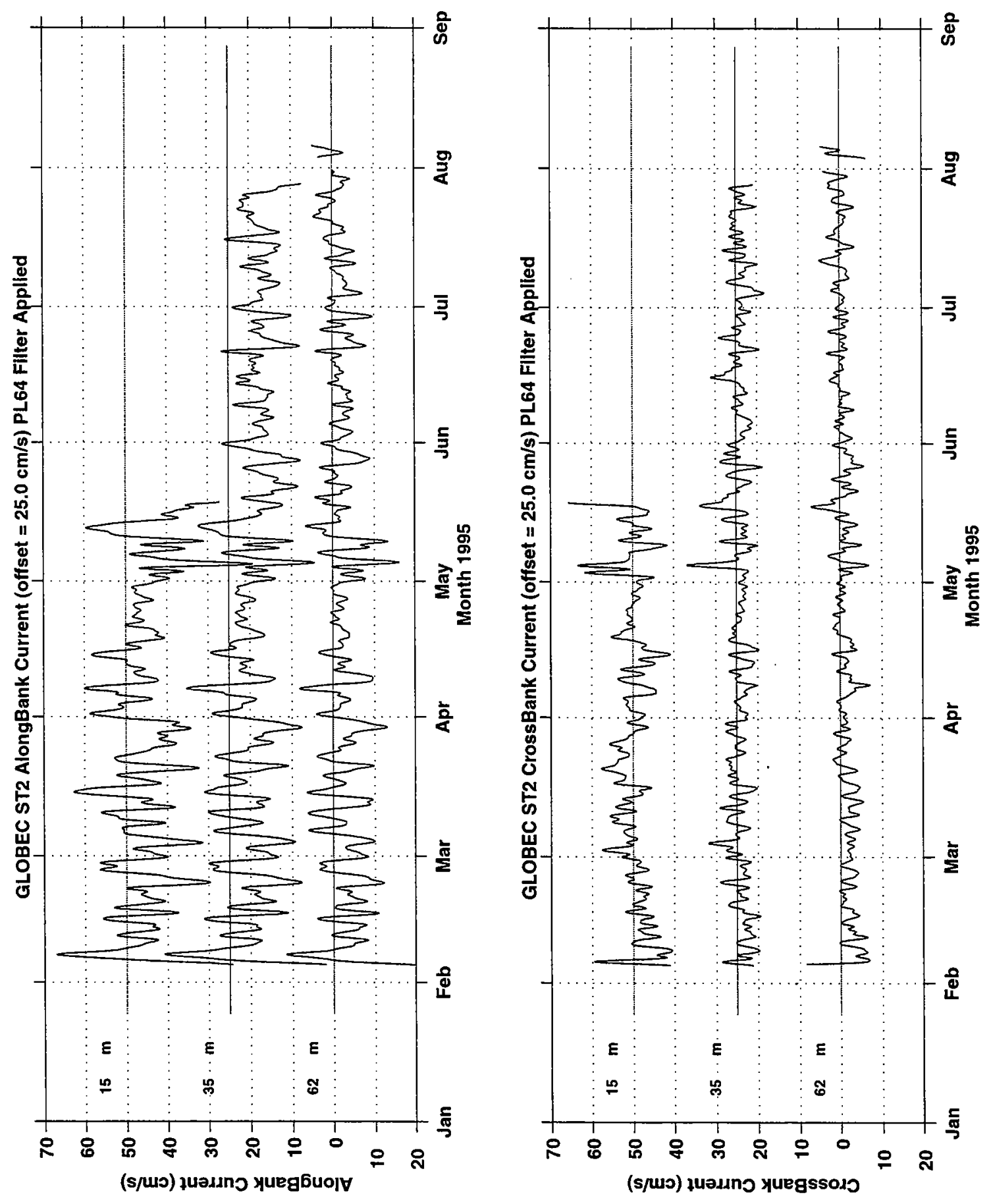

Figure 39 

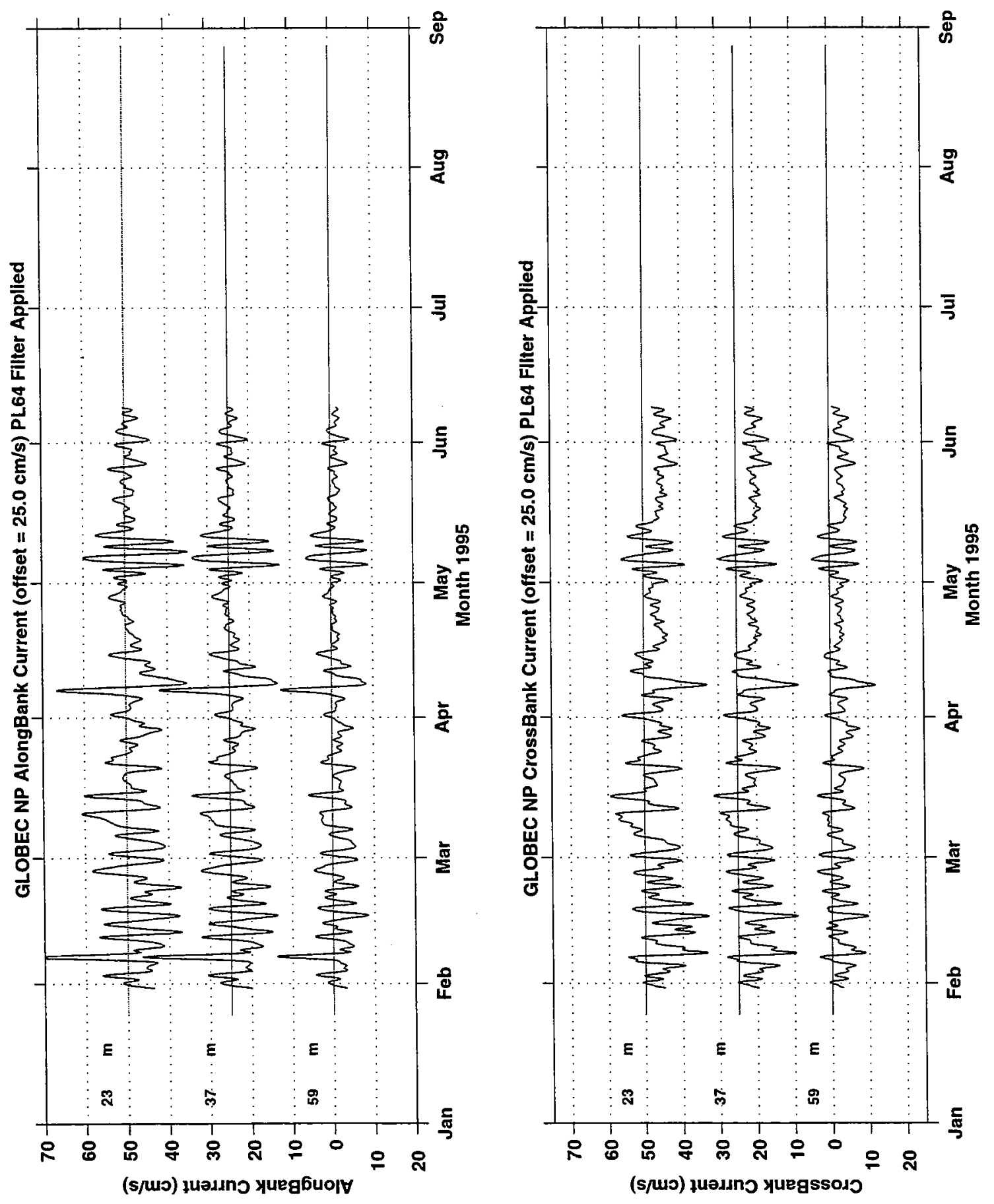

Figure 40 


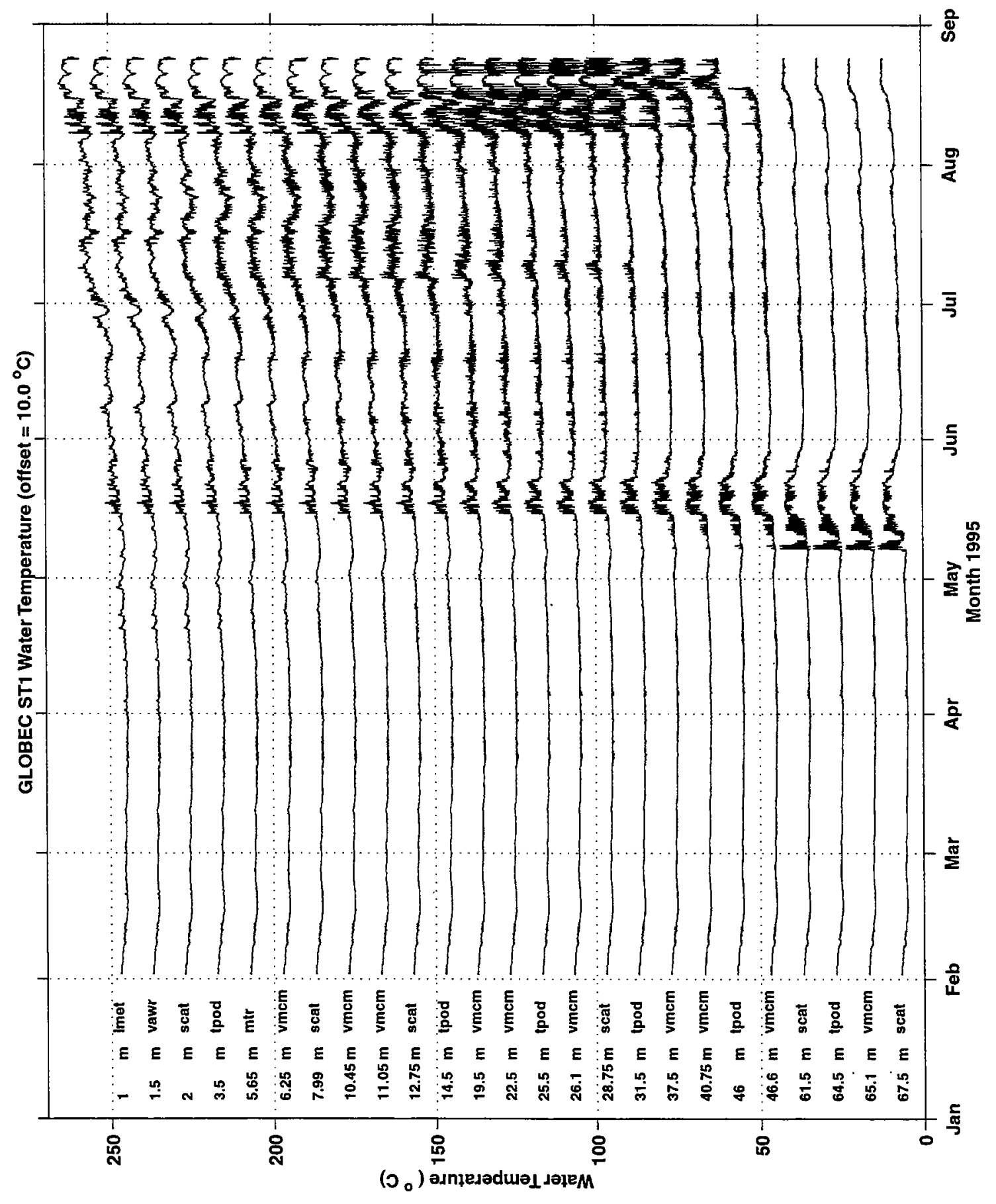

Figure 41 


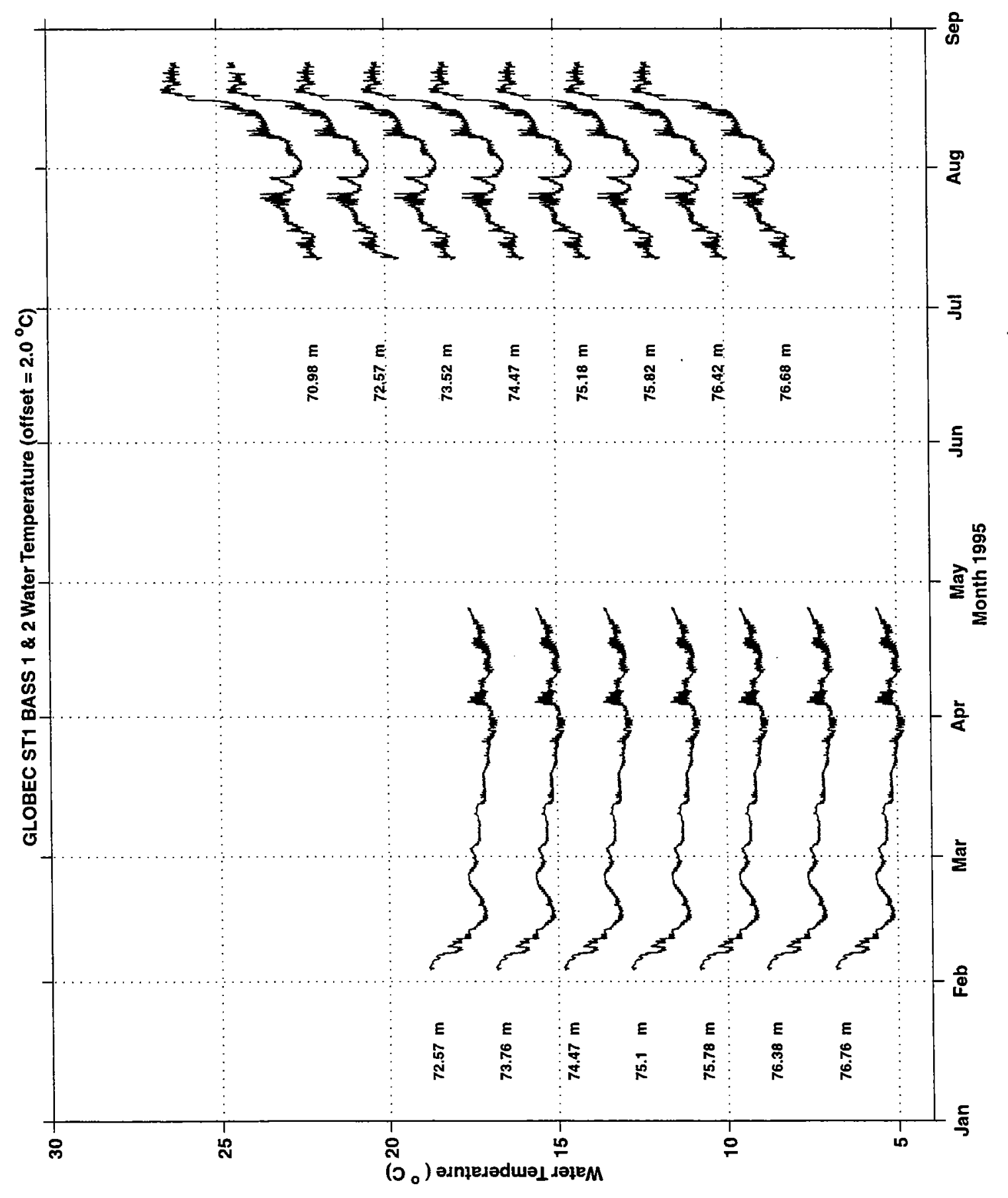

Figure 42 


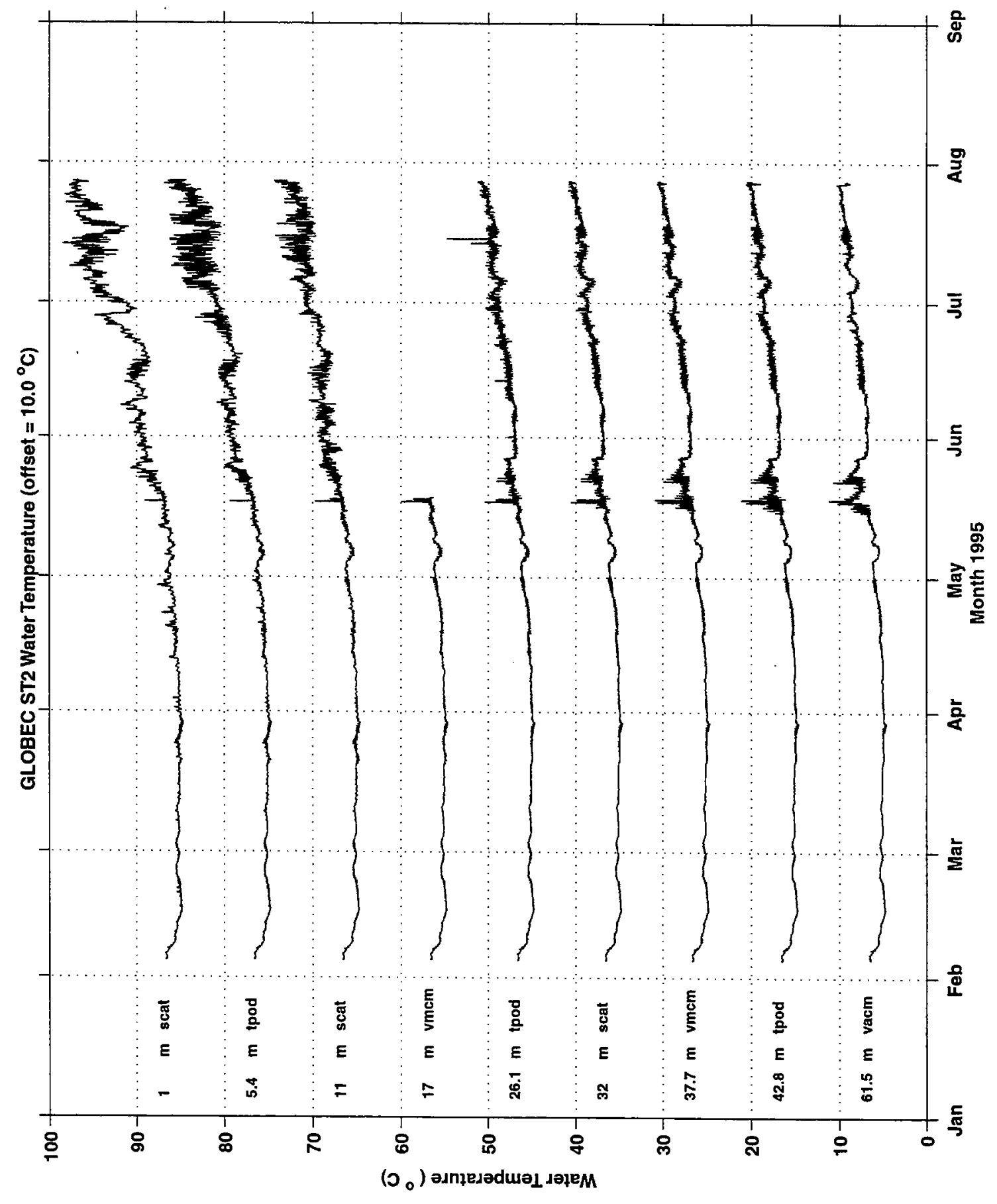

Figure 43 


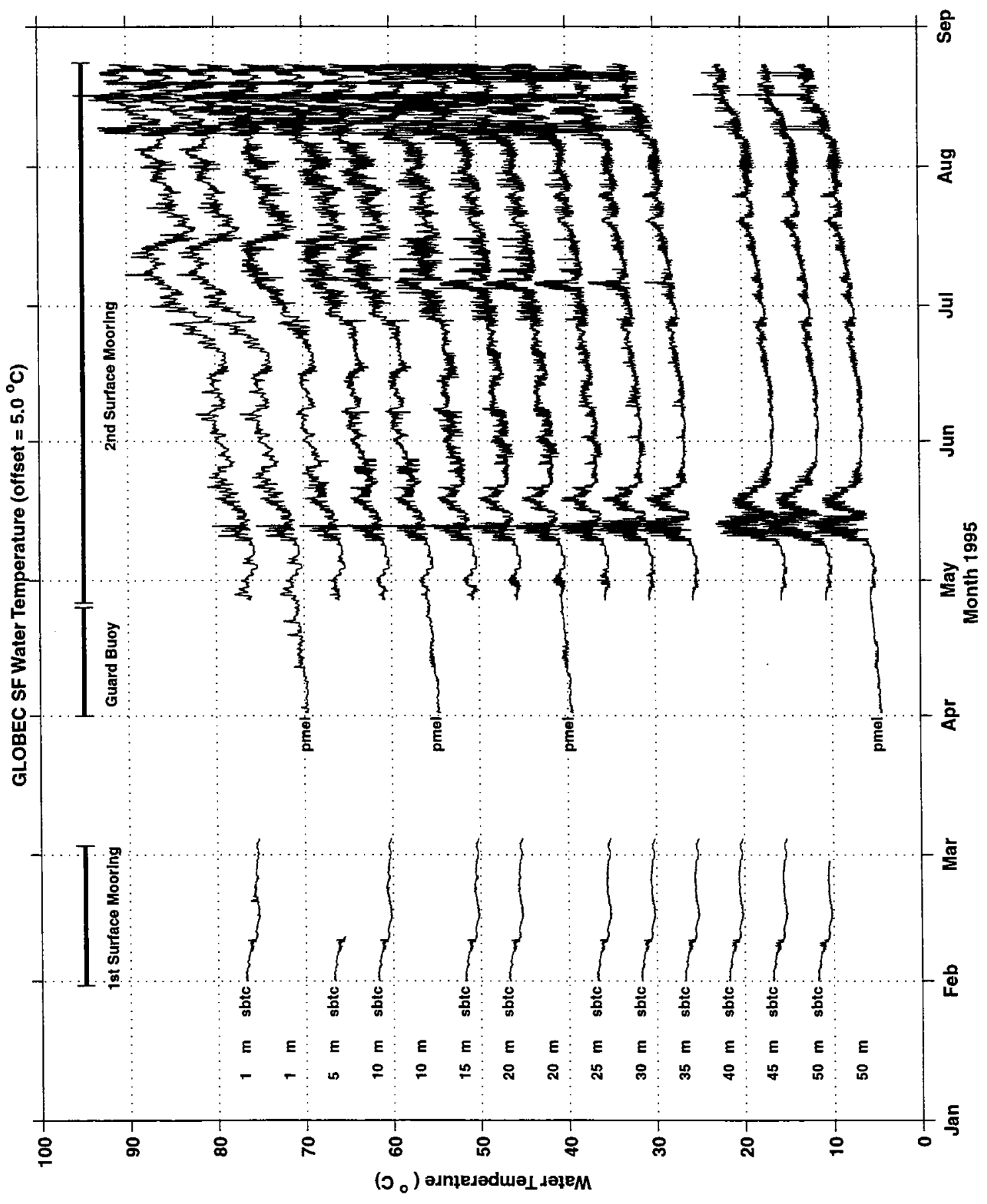

Figure 44 

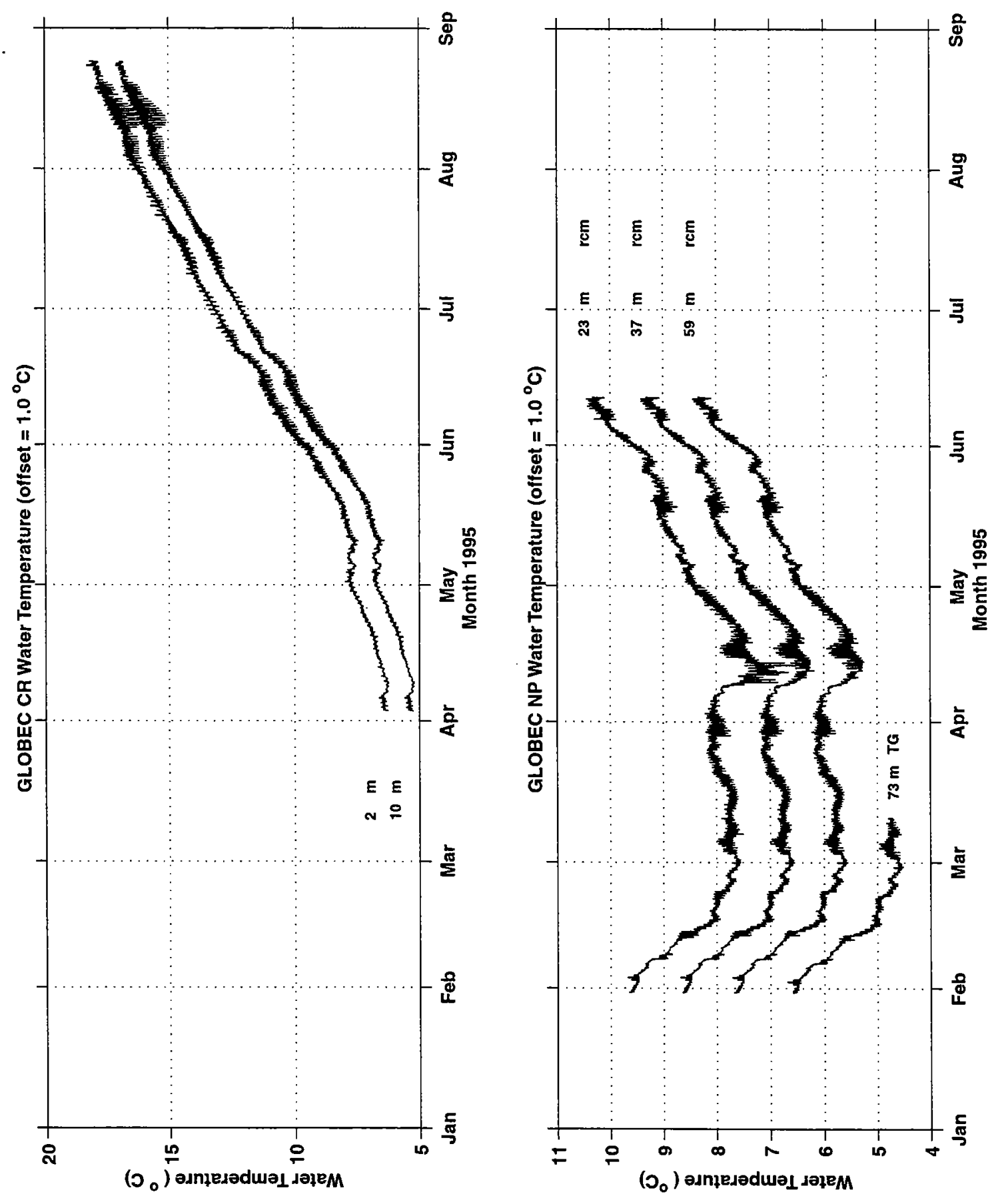

Figure 45 


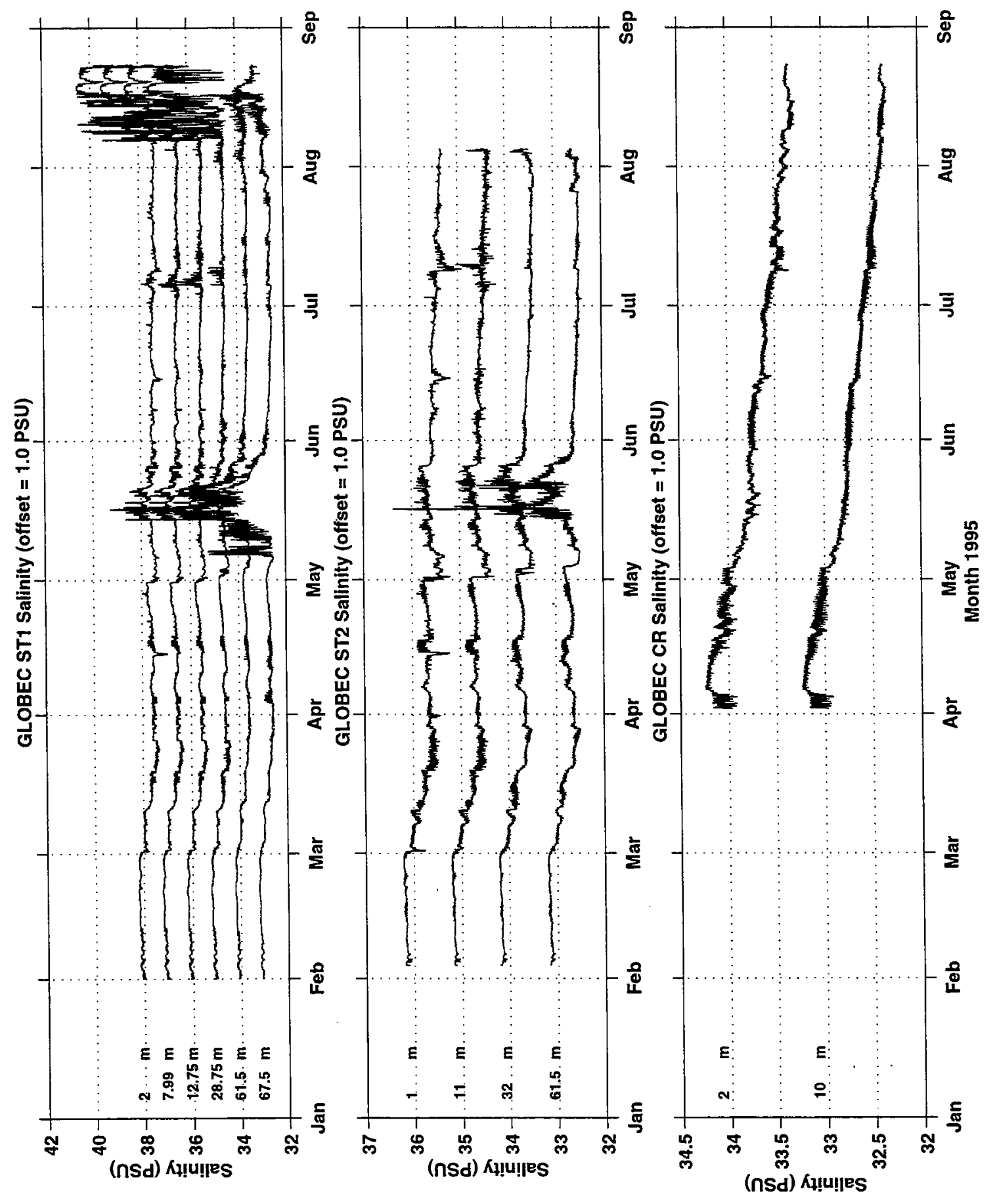

Figure 46 

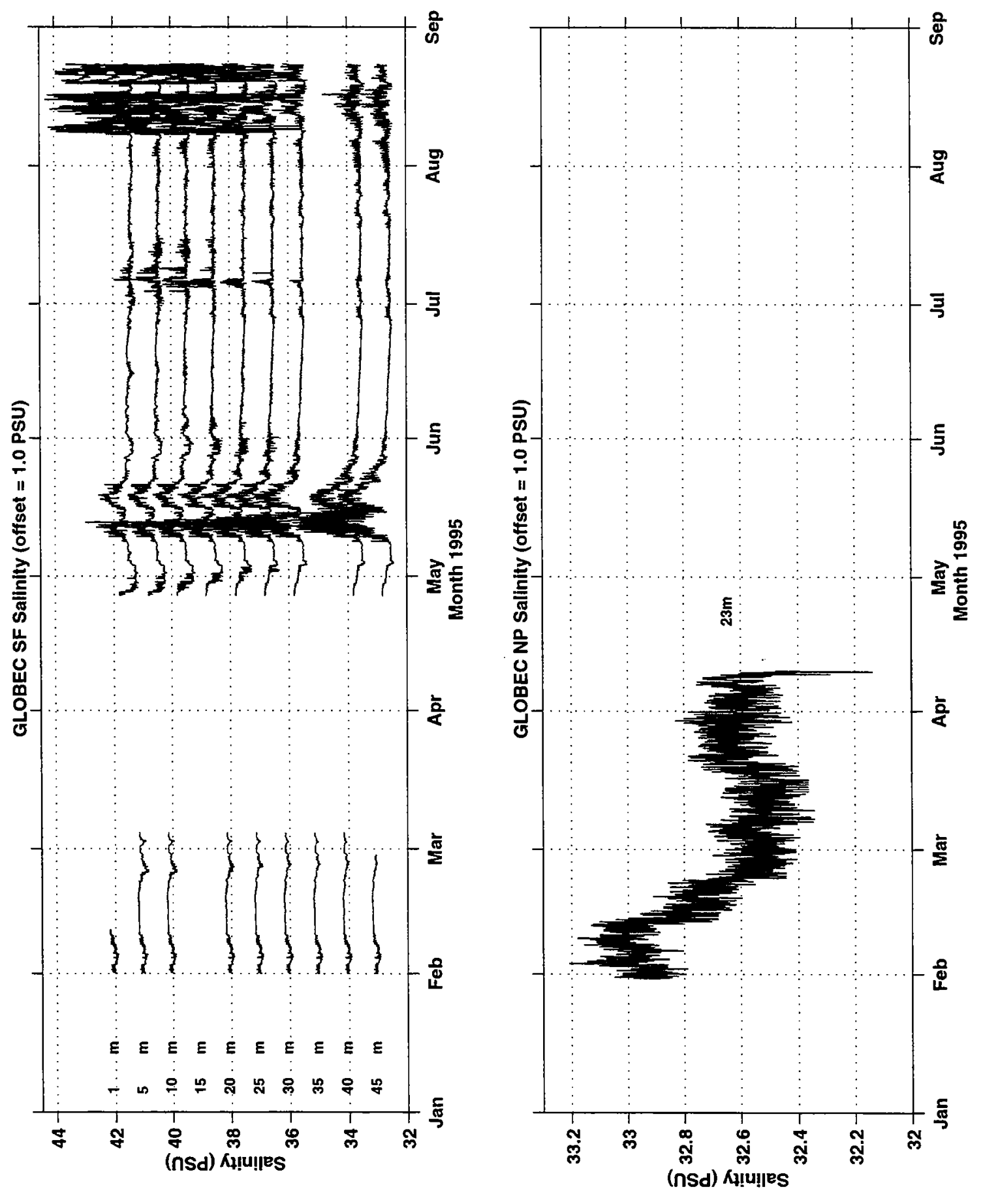

Figure 47 


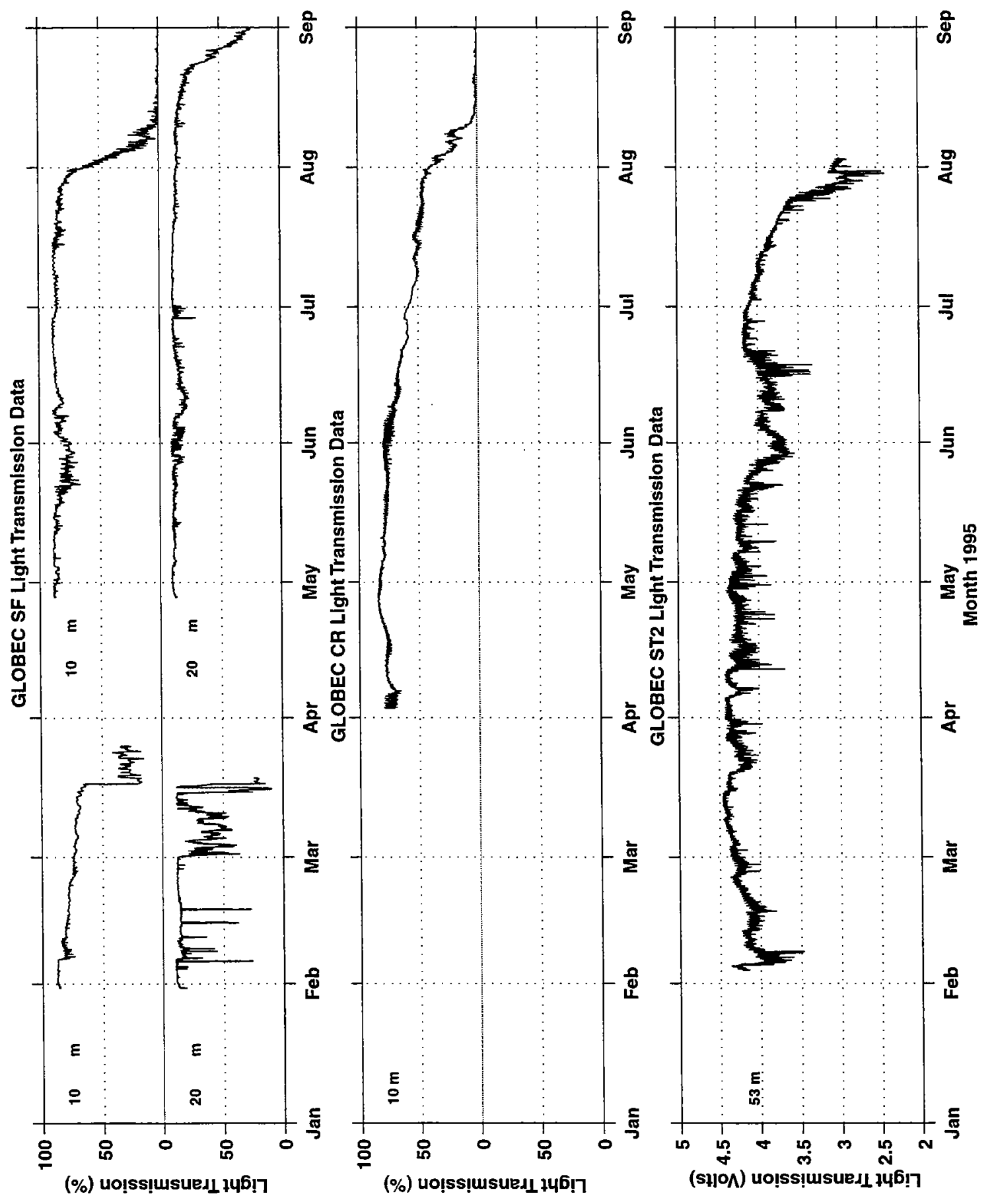

Figure 48 


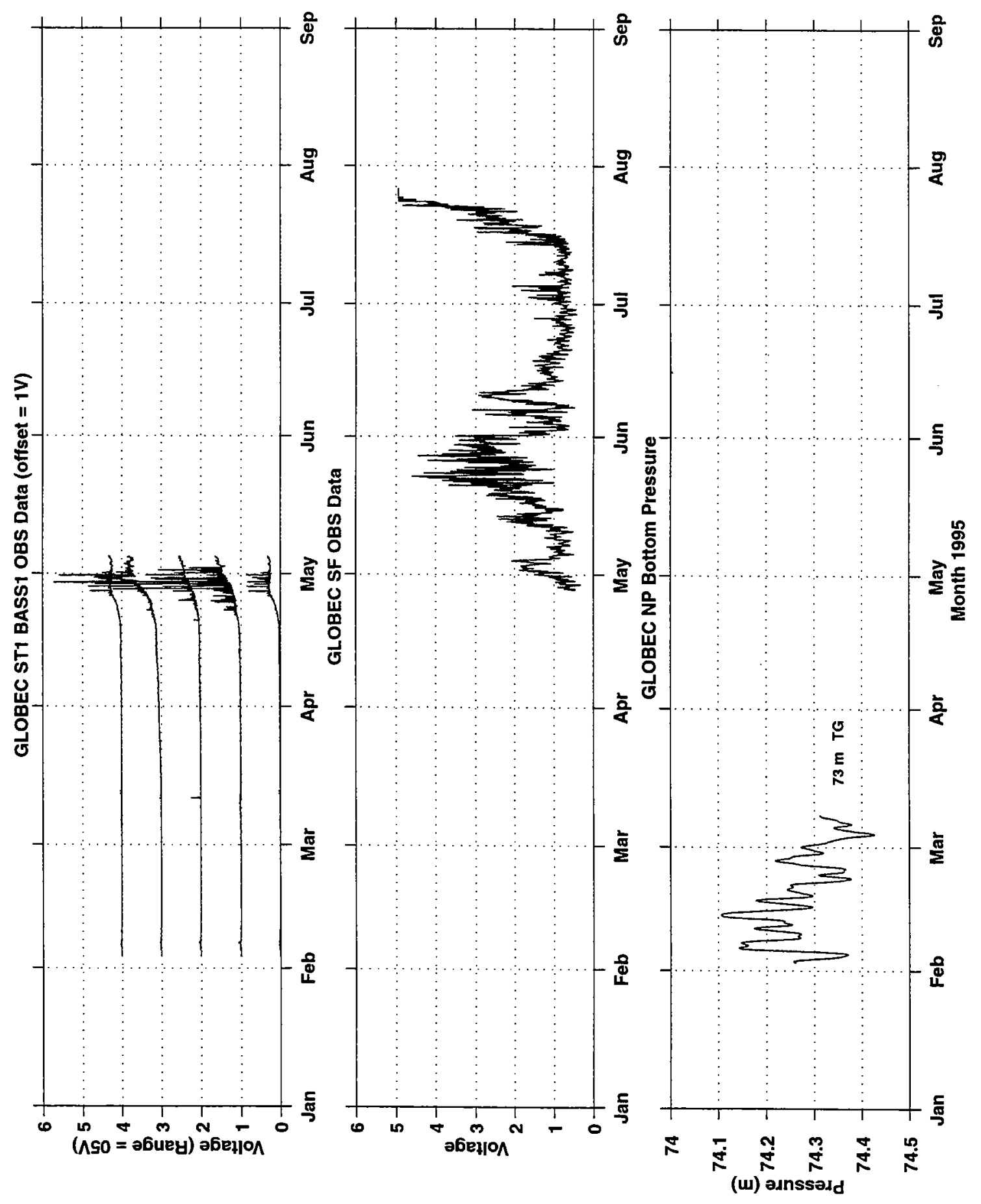

Figure 49 


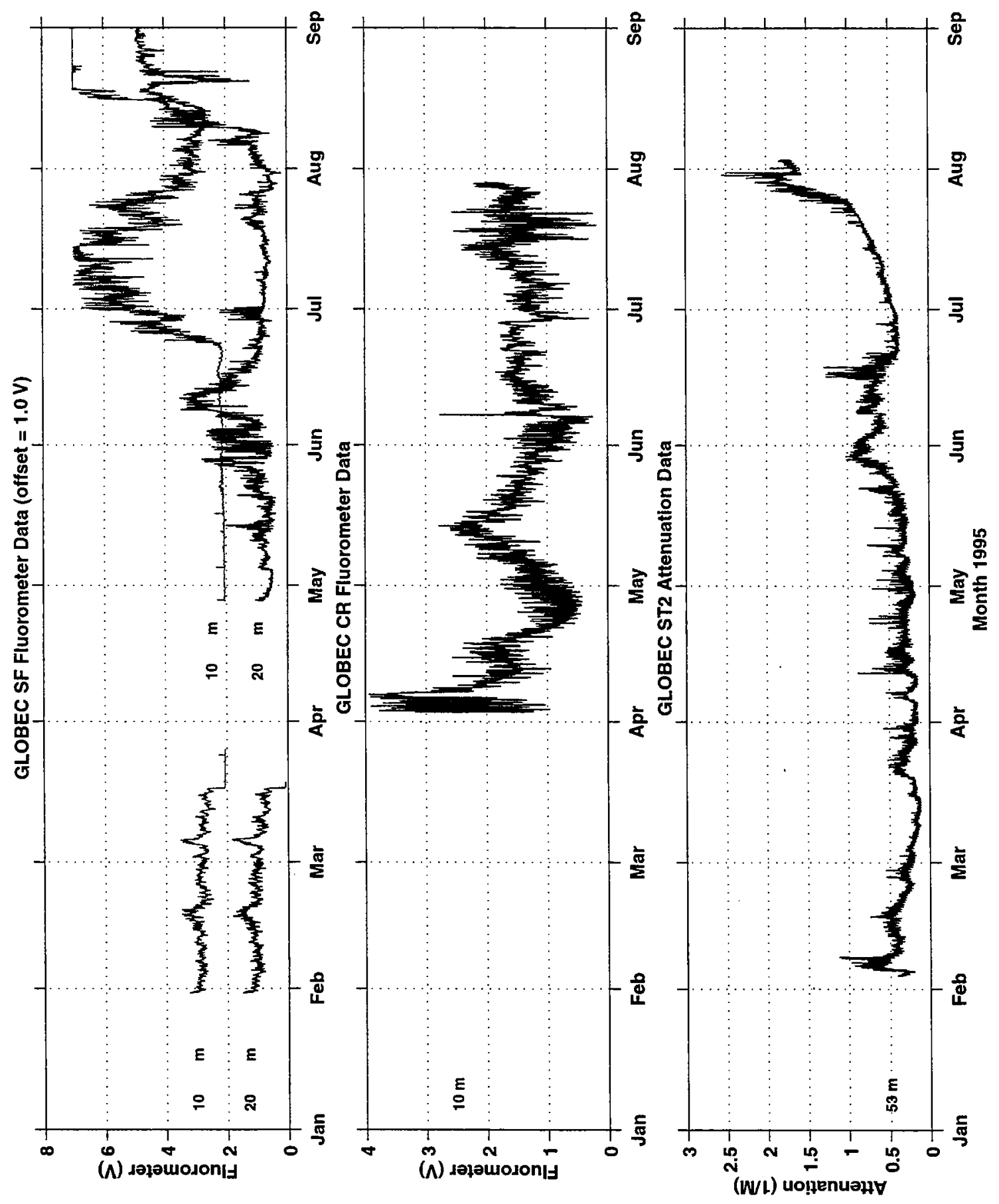

Figure 50 


\section{DOCUMENT LIBRARY}

Distribution List for Technical Report Exchange - July 1998

University of California, San Diego

SIO Library 0175C

9500 Gilman Drive

La Jolla, CA 92093-0175

Hancock Library of Biology \& Oceanography

Alan Hancock Laboratory

University of Southern California

University Park

Los Angeles, CA 90089-0371

Gifts \& Exchanges

Library

Bedford Institute of Oceanography

P.O. Box 1006

Dartmouth, NS, B2Y 4A2, CANADA

NOAA/EDIS Miami Library Center

4301 Rickenbacker Causeway

Miami, FL 33149

Research Library

U.S. Army Corps of Engineers

Waterways Experiment Station

3909 Halls Ferry Road

Vicksburg, MS 39180-6199

Marine Resources Information Center

Building E38-320

MIT

Cambridge, MA 02139

Library

Lamont-Doherty Geological Observatory

Columbia University

Palisades, NY 10964

Library

Serials Department

Oregon State University

Corvallis, OR 97331

Pell Marine Science Library

University of Rhode Island

Narragansett Bay Campus

Narragansett, RI 02882

Working Collection

Texas A\&M University

Dept. of Oceanography

College Station, TX 77843
Fisheries-Oceanography Library

151 Oceanography Teaching Bldg.

University of Washington

Seattle, WA 98195

Library

R.S.M.A.S.

University of Miami

4600 Rickenbacker Causeway

Miami, FL 33149

Maury Oceanographic Library

Naval Oceanographic Office

Building 1003 South

1002 Balch Blvd.

Stennis Space Center, MS, 39522-5001

Library

Institute of Ocean Sciences

P.O. Box 6000

Sidney, B.C. V8L 4B2

CANADA

National Oceanographic Library

Southampton Oceanography Centre

European Way

Southampton SO14 3ZH

UK

The Librarian

CSIRO Marine Laboratories

G.P.O. Box 1538

Hobart, Tasmania

AUSTRALIA 7001

Library

Proudman Oceanographic Laboratory

Bidston Observatory

Birkenhead

Merseyside L43 7 RA

UNITED KINGDOM

IFREMER

Centre de Brest

Service Documentation - Publications

BP 7029280 PLOUZANE

FRANCE 


\begin{tabular}{|c|c|c|}
\hline $\begin{array}{l}\text { REPORT DOCUMENTATION } \\
\text { PAGE }\end{array}$ & $\begin{array}{l}\text { 1. REPORT NO. } \\
\text { WHOI-2001-11 }\end{array}$ & 3. Recipient's Accession No. \\
\hline \multirow{2}{*}{\multicolumn{2}{|c|}{$\begin{array}{l}\text { 4. Title and Subtitie } \\
\text { The } 1995 \text { Georges Bank Stratification Study and Moored Array Measurements }\end{array}$}} & $\begin{array}{l}\text { 5. Report Date } \\
\text { August } 2001\end{array}$ \\
\hline & & 6. \\
\hline \multicolumn{2}{|c|}{$\begin{array}{l}\text { 7. Author(s) C. Alessi, R. Beardsley, M. Caruso, J. Churchill, J. Irish, S. Lentz, R. Limeburner, } \\
\text { R. Werner, R. Weller, A. Williams, W. Williams, J. Manning, P. Smith }\end{array}$} & $\begin{array}{l}\text { 8. Performing Organization Rept. No. } \\
\text { WHOI-2001-11 }\end{array}$ \\
\hline \multirow{2}{*}{\multicolumn{2}{|c|}{$\begin{array}{l}\text { 9. Performing Organization Name and Address } \\
\text { Woods Hole Oceanographic Institution } \\
\text { Woods Hole, Massachusetts } 02543\end{array}$}} & 10. Project/TaskWork Unit No. \\
\hline & & $\begin{array}{l}\text { 11. Contract(C) or Grant(G) No. } \\
\text { (C) OCE-98-06379 } \\
\text { (G) OCE-98-06445 }\end{array}$ \\
\hline \multirow{2}{*}{\multicolumn{2}{|c|}{$\begin{array}{l}\text { 12. Sponsoring Organization Name and Address } \\
\text { National Science Foundation }\end{array}$}} & $\begin{array}{l}\text { 13. Type of Report \& Period Covered } \\
\text { Technical Report }\end{array}$ \\
\hline & & 14. \\
\hline
\end{tabular}

15. Supplementary Notes

This report should be cited as: Woods Hole Oceanog. Inst. Tech. Rept., WHOI-2001-11.

16. Abstract (Limit: 200 words)

The 1995 Georges Bank Stratification Study (GBSS) was the first intensive process study conducted as part of the U.S. GLOBEC Northwest Atlantic/Georges Bank field program. The GBSS was designed to investigate the physical processes which control the seasonal development of stratification along the southern flank of Georges Bank during spring and summer. Past work suggested that during this period, larval cod and haddock tended to aggregate to the thermocline on the southern flank where higher concentrations of their copepod prey were found. A moored array was deployed as part of the GBSS to observe the onset and evolution of seasonal stratification over the southern flank with sufficient vertical and horizontal resolution that key physical processes could be identified and quantified. Moored current, temperature, and conductivity (salinity) measurements were made at three sites on the southern flank, one on the crest, and one on the northeast peak of the bank. Moored surface meteorological measurements were also make at one southern flank site ot determine the surface wind stress and heat and moisture fluxes. The oceanographic and meteorological data collected with the GBSS array during January-August, 1995 are presented in this report. Meteorological data collected on National Data Buoy Center environmental buoys 44011 (Georges Bank), 44008 (Nantucket Shoals), and 44005 (Gulf of Maine) are included in this report for completeness and comparison with the GBSS southern flank meteorological measurements.

17. Document Analysis a. Descriptors US GLOBEC

Georges Bank

moored array

b. Identifiers/Open-Ended Terms

c. COSATI Field/Group

18. Availability Statement

Approved for public release; distribution unlimited.

\begin{tabular}{|c|}
$\begin{array}{c}\text { 19. Security Class (This Report) } \\
\text { UNCLASSIFIED }\end{array}$ \\
\hline 20. Security Class (This Page) \\
\hline
\end{tabular}

21. No. of Pages 113

22. Price 